Florida International University FIU Digital Commons

$5-17-2013$

\title{
Electrochemical Immunosensing of Cortisol in an Automated Microfluidic System Towards Point-of- Care Applications
}

Abhay Vasudev

avasu002@fiu.edu

DOI: $10.25148 /$ etd.FI13080720

Follow this and additional works at: https:// digitalcommons.fiu.edu/etd

Part of the Biomedical Commons, Biomedical Devices and Instrumentation Commons, Nanoscience and Nanotechnology Commons, and the Nanotechnology Fabrication Commons

\section{Recommended Citation}

Vasudev, Abhay, "Electrochemical Immunosensing of Cortisol in an Automated Microfluidic System Towards Point-of-Care Applications" (2013). FIU Electronic Theses and Dissertations. 956.

https://digitalcommons.fiu.edu/etd/956 


\title{
FLORIDA INTERNATIONAL UNIVERSITY
}

Miami, Florida

\section{ELECTROCHEMICAL IMMUNOSENSING OF CORTISOL IN AN AUTOMATED MICROFLUIDIC SYSTEM TOWARDS POINT-OF-CARE APPLICATIONS}

\author{
A dissertation submitted in partial fulfillment of \\ the requirements for the degree of \\ DOCTOR OF PHILOSOPHY \\ in \\ ELECTRICAL ENGINEERING \\ by
}

Abhay Vasudev

2013 
To: Dean Amir Mirmiran

College of Engineering and Computing

This dissertation, written by Abhay Vasudev, and entitled Electrochemical Immunosensing of Cortisol in an Automated Microfluidic System towards Point-of-Care Applications, having been approved in respect to style and intellectual content, is referred to you for judgment.

We have read this dissertation and recommend that it be approved.

$\begin{array}{r}\hline \text { Nezih Pala } \\ \hline \text { Irene Calizo } \\ \hline \text { Wei-Chiang Lin }\end{array}$

$\begin{array}{r}\hline \text { Kinzy Jones } \\ \hline \text { Joong-ho Moon } \\ \hline \text { Shedra Amy Snipes }\end{array}$

Shekhar Bhansali, Major Professor

Date of Defense: May 17, 2013

The dissertation of Abhay Vasudev is approved.

Dean Amir Mirmiran College of Engineering and Computing

Dean Lakshmi N. Reddi University Graduate School

Florida International University, 2013 
C Copyright 2013 by Abhay Vasudev

All rights reserved. 


\section{DEDICATION}

To My Parents

Smt. Champa Vasudev and Dr. M. J. Vasudeva Rao 


\section{ACKNOWLEDGMENTS}

First and foremost, I would like to express gratitude to my advisor and mentor, Dr. Shekhar Bhansali, for giving me the opportunity to work on an exciting project for my doctoral dissertation. I am also thankful for his encouragement and support in developing my research skills and personality through the many opportunities provided during my time at Florida International University and University of South Florida.

I would like to thank the members of my dissertation committee Dr. Nezih Pala, Dr. Irene Calizo, Dr. Wei-Chiang Lin, Dr. Joong-ho Moon, Dr. Shedra Amy Snipes and Dr. Kinzy Jones, for agreeing to be my dissertation committee, and for their valuable inputs towards my dissertation research. In particular, I would like to thank Dr. Lin for his help and support during my transition to Florida International University. I would also like to thank Dr. Jones for his valuable inputs towards the LTCC microfluidic device fabrication and for access to the LTCC lab and other facilities at the Advanced Materials Engineering Research Institute (AMERI).

I thank the Department of Electrical and Computer Engineering, and the Department of Biomedical Engineering for the logistical support. I also immensely thank Ms. Pat Brammer and Ms. Ana Seinz for their administrative support. I would like to thank the Graduate \& Professional Student Committee (GPSC) for the support received through conference travel grants to present my research at many national and international conferences.

I take this opportunity to also thank all the former and present members of the bio-MEMS and Microsystems Lab spread across Florida International University and University of South Florida. In particular, Dr. Ajeet Kaushik for his mentorship and 
scientific inputs, Dr. Subramanian Krishnan for his encouragement and support, Dr. Yuichi Tomizawa for introducing and guiding me on the simulations and Dr. Sunil Arya for always being there for any technical inputs on the biosensor design. Many thanks to my fellow graduate students, Mr. Eric Huey, Mr. Kevin Luongo, Mr. Frank Alexander, Mr. Justin Boone, Mr. Khalid Pasha, Ms. Aparajita Singh, Ms. Angela Colucci and Ms. Angie Marie Patricia who have accompanied me and supported me during my graduate student days. I would like to thank all the undergraduate researchers in our lab, Nicolas Norena, Adrian Martin, Andres Felipe Diaz, Randy Matos, Juan Espinosa and Justin Kalap. I would like to thank Mr. Rupak Dua for helping me on the ELISA assays and for access to the ELISA plate reader. To all my other colleagues, collaborators and friends at USF and FIU, whose names would be too long to enumerate here: thank you for creating such a great working environment and making learning a fun task!

I would like to acknowledge the funding sources that made this work possible: the National Institute of Health (NIH), The ASSIST-NERC program from the National Science Foundation (NSF), The Department of Electrical and Computer Engineering and the Department of Biomedical Engineering at Florida International University.

I want to thank my friends at Tampa and Miami for their support, love and encouragement. A million thanks to my friend Mr. Akshay Mudlapur for being a morale booster when the chips were down. Finally, I could not have come this far without the unconditional love, support and encouragement and blessings from my family. Many thanks to my father, who has been my role model, my mother for her unconditional love and support and my sister for her constant encouragement. Many thanks to my wife Siri for the constant encouragement, support and love during my student days. 


\title{
ABSTRACT OF THE DISSERTATION \\ ELECTROCHEMICAL IMMUNOSENSING OF CORTISOL IN AN AUTOMATED MICROFLUIDIC SYSTEM TOWARDS POINT-OF-CARE APPLICATIONS
}

\author{
by
}

Abhay Vasudev

Florida International University, 2013

Miami, Florida

\section{Professor Shekhar Bhansali, Major Professor}

This dissertation describes the development of a label-free, electrochemical immunosensing platform integrated into a low-cost microfluidic system for the sensitive, selective and accurate detection of cortisol, a steroid hormone co-related with many physiological disorders. Abnormal levels of cortisol is indicative of conditions such as Cushing's syndrome, Addison's disease, adrenal insufficiencies and more recently posttraumatic stress disorder (PTSD). Electrochemical detection of immuno-complex formation is utilized for the sensitive detection of Cortisol using Anti-Cortisol antibodies immobilized on sensing electrodes. Electrochemical detection techniques such as cyclic voltammetry (CV) and electrochemical impedance spectroscopy (EIS) have been utilized for the characterization and sensing of the label-free detection of Cortisol. The utilization of nanomaterial's as the immobilizing matrix for Anti-cortisol antibodies that leads to improved sensor response has been explored. A hybrid nano-composite of Polyanaline$\mathrm{Ag} / \mathrm{AgO}$ film has been fabricated onto Au substrate using electrophoretic deposition for the preparation of electrochemical immunosening of cortisol. Using a conventional 3electrode electrochemical cell, a linear sensing range of $1 \mathrm{pM}$ to $1 \mu \mathrm{M}$ at a sensitivity of 
$66 \mu \mathrm{A} / \mathrm{M}$ and detection limit of $0.64 \mathrm{pg} / \mathrm{mL}$ has been demonstrated for detection of cortisol. Alternately, a self-assembled monolayer (SAM) of dithiobis(succinimidylpropionte) (DTSP) has been fabricated for the modification of sensing electrode to immobilize with Anti-Cortisol antibodies. To increase the sensitivity at lower detection limit and to develop a point-of-care sensing platform, the DTSP-SAM has been fabricated on micromachined interdigitated microelectrodes ( $\mu$ IDE). Detection of cortisol is demonstrated at a sensitivity of $20.7 \mu \mathrm{A} / \mathrm{M}$ and detection limit of $10 \mathrm{pg} / \mathrm{mL}$ for a linear sensing range of $10 \mathrm{pM}$ to $200 \mathrm{nM}$ using the $\mu \mathrm{IDE}$ 's.

A simple, low-cost microfluidic system is designed using low-temperature cofired ceramics (LTCC) technology for the integration of the electrochemical cortisol immunosensor and automation of the immunoassay. For the first time, the non-specific adsorption of analyte on LTCC has been characterized for microfluidic applications. The design, fabrication technique and fluidic characterization of the immunoassay are presented. The DTSP-SAM based electrochemical immunosensor on $\mu$ IDE is integrated into the LTCC microfluidic system and cortisol detection is achieved in the microfluidic system in a fully automated assay. The fully automated microfluidic immunosensor hold great promise for accurate, sensitive detection of cortisol in point-of-care applications. 


\section{TABLE OF CONTENTS}

CHAPTER

PAGE

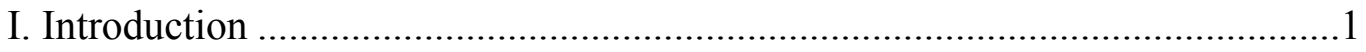

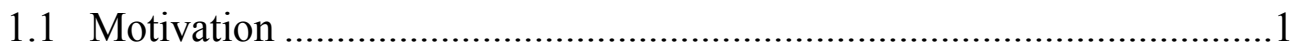

1.2 Specific Aims .................................................................................

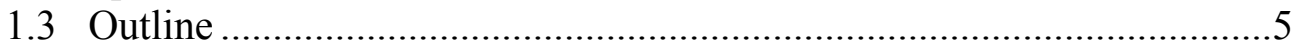

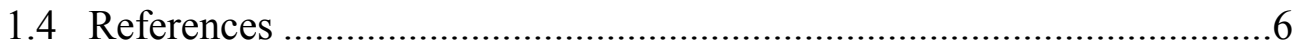

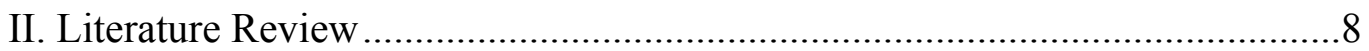

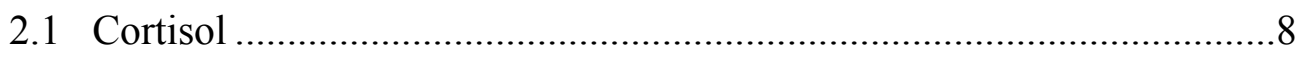

2.1.1 Secretion of Cortisol........................................................... 9

2.1.2 Source of Sample ........................................................... 11

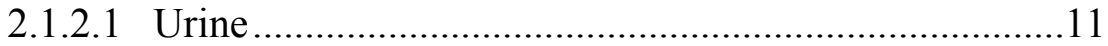

2.1.2.2 Interstitial Fluid (ISF) ............................................13

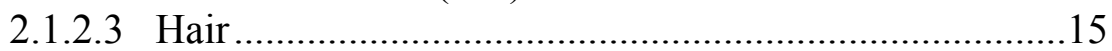

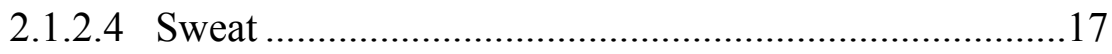

2.1.2.1 Blood ..................................................................... 17

2.1.2.1 Saliva ................................................................... 18

2.1.3 Detection of Cortisol: State of the art .....................................20

2.1.3.1 High Performance Liquid Chromatography (HPLC) ....20

2.1.3.2 Immunoassays ......................................................20

2.1.3.3 Electrochemical Immunoassays ...............................24 2.1.3.3.1 Nanomaterials .............................................32

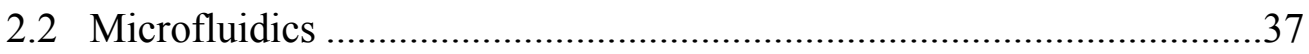

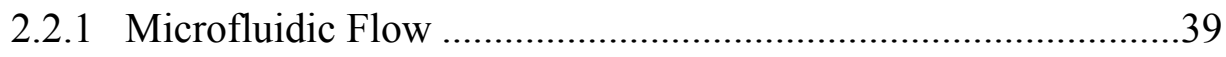

2.2.2 Types of Microfluidic Flow ..................................................40

2.2.3 Fabrication of Microfluidic Devices .......................................43

2.2.4 Low Temperature Co-fired Ceramics (LTCC)..........................47

2.2.4.1 LTCC Processing ...................................................50

2.2.4.2 Fabrication of Microfluidic Components in LTCC.......52

2.2.4.3 Applications of LTCC Microfluidic Systems ...............57

2.3 References

III. Electrophoretically Deposited Polyanaline-Ag/AgO Hybrid Nanocomposite Based Electrochemical Immunosensor for Mediator-Free Detection of Cortisol

3.1 Organic-Inorganic Hybrid Nanocomposites.........................................84

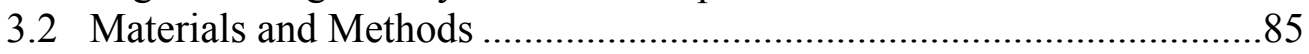

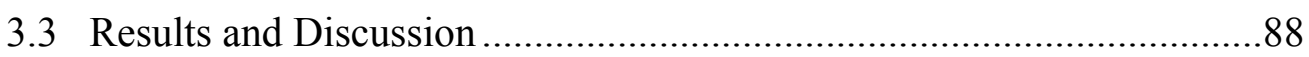

3.3.1 Optical Characterization of $\mathrm{Ag} @ \mathrm{AgO} \mathrm{NP} \& \mathrm{Ag} @ \mathrm{AgO}-\mathrm{PANI}$

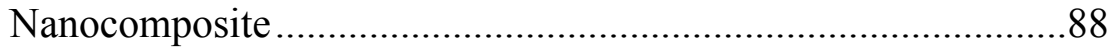

3.3.2 Electrochemical Characterization …..................................91 
3.3.3 Electrochemical Response Study for Cortisol Detection 100

3.4 Conclusion

IV. Dithiobis (succinimidylpropionte) (DTSP) Modified Interdigitated Microelectrodes $(\mu \mathrm{IDE})$ for Electrochemical Immunosensing of

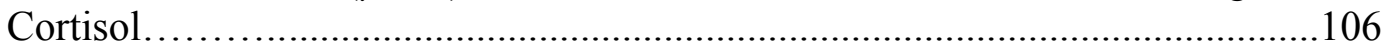

4.1 Self Assembled Monolayers (SAM) ..................................................106

4.2 Dithiobis (succinimidylpropionte) (DTSP) ………...............................107

4.3 Interdigitated Microelectrode ( $\mu$ IDE) Design .........................................108

$4.4 \mu \mathrm{IDE}$ Fabrication Process ................................................................... 110

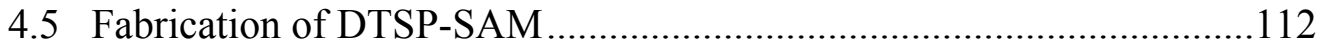

4.3.1 Electrochemical Characterization .............................................113

4.6 Functionalization of $\mu$ IDE for Cortisol Detection ...................................114

4.7 Electrochemical Immunosensing Studies of Cortisol.............................120

4.8 Cortisol Detection in Saliva Samples .................................................124

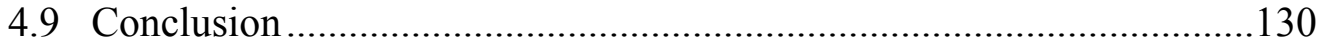

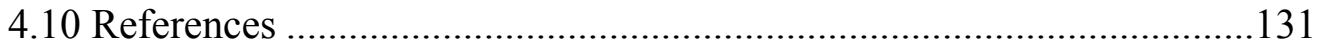

V. Low Temperature Co-fired Ceramics (LTCC) Based Microfluidic System for Automation of Cortisol Immunoassay ................................................................133

5.1 Non-Specific Adsorption (NSA) of Cortisol on LTCC ...........................133

5.1.1 Design of Experiment, Materials and Methods............................136

5.1.2 Results and Discussion.............................................................137

5.2 Microfluidic Protocol for Electrochemical Immunosensing .................143

5.3 Design Optimization Using Computational Fluid Dynamics (CFD) ....144

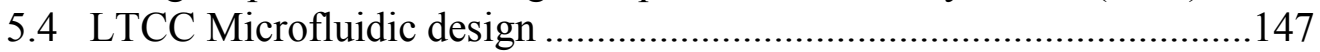

5.4.1 Fabrication of LTCC based Microfluidic Chip ...........................148

5.4.2 Fluidic Characterization in LTCC Microfluidic Chip.................149

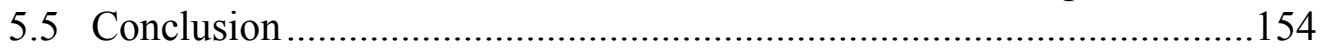

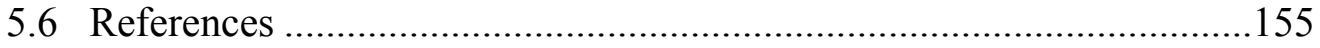

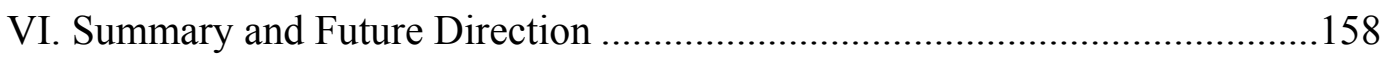

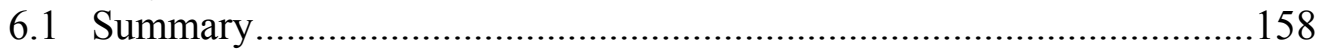

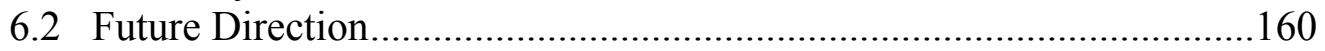

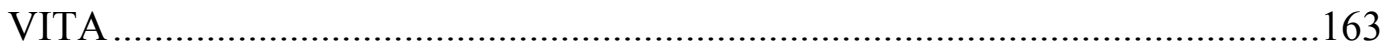




\section{LIST OF FIGURES}

FIGURE

PAGE

2.1 Chemical structure of cortisol; (a) 2D and (b) 3D...............................

2.2 The typical diurnal variation of cortisol levels over a 24-hour cycle. .......9

2.3 Cortisol secretion regulated by the HPA axis .......................................11

2.4 (a) Cross-section of micropore generated through laser ablation; (b) Four pores relative to the size of a penny; (c) Handheld laser source used to create micropores...................................................................... 14

2.5 Schematic illustration of the accumulation of cortisol in the medulla of a hair follicle .16

2.6 Schematic illustrations of various immunoassay techniques..................22

2.7 Schematic of strategies used for electrochemical immunosensing ...........26

2.8 A typical cyclic voltammogram with defined characteristics.................29

2.9 Electrochemical impedance spectroscopy data represented in terms of (a) Nyquist plot and (b) Bode plot .32

2.10 Schematic illustration of flow profiles seen in (a) Pressure driven flow and (b) Electro-osmotic flow

2.11 Schematic illustration of electrowetting of droplet for achieving droplet transport on individually addressable electrodes

2.12 Schematic illustration of the three basic processing steps in microfabrication

2.13 Schematic illustration of the processing steps in fabrication of PDMS structures using soft lithography

2.14 (a) Schematic illustration of the processing steps in LTCC based microfabrication; (b) Temperature ramp profile during the sintering process of LTCC structures

3.1 Schematic illustration of the two-electrode electrochemical cell for electrophoretic deposition..... 87 
3.2 (a) TEM image of core-shell Ag@AgO nanoparticles high resolution, (b) image of Ag@AgO nanoparticles, and (c) EDX spectra of Ag@AgO nanoparticles

3.3 (a) AFM image of $\mathrm{Ag} @ \mathrm{AgO}-\mathrm{PANI} / \mathrm{Au}$ nanocomposite, (b) EDX spectra of Ag@AgO-PANI/Au nanocomposite, and (c) BSA/Anti-C $\mathrm{ab}_{\mathrm{ab}} / \mathrm{Ag} @ \mathrm{AgO}-$ $\mathrm{PANI} / \mathrm{Au}$ immunoelectrode 90

3.4 Characterization of stepwise fabrication of nanocomposite and immunosensor using EIS in PBS $(10 \mathrm{nM}, \mathrm{pH}=7,0.9 \% \mathrm{NaCl})$ containing $5 \mathrm{mM} \mathrm{Fe}(\mathrm{II}) / \mathrm{Fe}(\mathrm{III})$ redox moieties .92

3.5 Characterization of stepwise fabrication of nanocomposite and immunosensor using $\mathrm{CV}$ in PBS $(10 \mathrm{nM}, \mathrm{pH}=7,0.9 \% \mathrm{NaCl})$; inset: $\mathrm{CV}$ of bare Au electrode 94

3.6 CV studies of immunoelectrode as a function of $\mathrm{pH}$ (6.0 to 8.0); inset: (a) magnitude of current v/s $\mathrm{pH}$ for BSA/Anti-C $\mathrm{ab}_{\mathrm{ab}} / \mathrm{Ag} @ \mathrm{AgO}-\mathrm{PANI} / \mathrm{Au}$ immunoelectrode; (b) CV studies of $\mathrm{Ag} @ \mathrm{AgO}-\mathrm{PANI} / \mathrm{Au}$ nanocomposite electrode .96

3.7 CV studies of $\mathrm{Ag} @ \mathrm{AgO}-\mathrm{PANI} / \mathrm{Au}$ electrode as a function of scan rate (10-100mV/s); inset: (a) magnitude of current response v/s square root of scan rate; (b) Redox potential v/s scan rate .98

3.8 CV studies of BSA/Anti- $\mathrm{C}_{\mathrm{ab}} / \mathrm{Ag} @ \mathrm{AgO}-\mathrm{PANI} / \mathrm{Au}$ electrode as a function of scan rate $(10-100 \mathrm{mV} / \mathrm{s})$; inset; (a) magnitude of current response $\mathrm{v} / \mathrm{s}$ square root of scan rate; (b) redox potential $\mathrm{v} / \mathrm{s}$ scan rate.

3.9 Electrochemical response studies of BSA/Anti- $\mathrm{C}_{\mathrm{ab}} / \mathrm{Ag} @ \mathrm{AgO}-\mathrm{PANI} / \mathrm{Au}$ immunoelectrode as function of cortisol concentration (1pM to $1 \mu \mathrm{M})$ using PBS ( $\mathrm{pH}$ 6.5, 0.9\% NaCl); inset: calibration curve between magnitude of response current and logarithm of cortisol concentration... 101

4.1 Three-dimensional molecular assembly of DTSP 108

4.2 (a) Schematic illustration of improved redox cycling in interdigitated microelectrodes; (b) Interdigitated Microelectrode design. 110

4.3 Schematic illustration of the microfabrication process flow for fabrication of the interdigitated microelectrodes 
4.4 Microfabricated interdigitated electrode chip (Left); Microscopic image of the interdigitated fingers (Right)

4.5 Schematic illustration of fabrication of DTSP-SAM on Au electrode ....113

4.6 Electrochemical characterization of DTSP-SAM formation using electrochemical impedance spectroscopy (EIS) .....................................114

4.7 Schematic of IDE functionalization with Anti-Cortisol antibodies .........115

4.8 The CV curves on the bare Au electrode (curve a), DTSP/Au electrode (curve b), Anti-Cab/DTSP/Au immunoelectrode (curve c), and EA/Anti$\mathrm{Cab} / \mathrm{DTSP} / \mathrm{Au}$ immunoelectrode (curve d) in have been carried out in PBS (50mM, pH 7.4 containing 5mM $\left.[\mathrm{Fe}(\mathrm{CN}) 6]^{3}{ }^{14}-\right)$..................................116

4.9 Electrochemical optimization as a function of $\mathrm{pH}$................................117

4.10 A) CV studies of DTSP/Au electrode as a function of scan rate (20-200 $\mathrm{mV} / \mathrm{s}$ ); inset a) magnitude of current response as a function of square root of scan rate, inset b) potential difference versus scan rate. B) CV studies of Anti-Cab/DTSP/Au immunoelectrode as a function of scan rate (20-200 $\mathrm{mV} / \mathrm{s}$ ); inset a) magnitude of current response as a function of square root of scan rate; inset b) potential difference versus scan rate.

4.11 EA/Anti-Cab/DTSP/Au electrode incubated with $10 \mu \mathrm{L}$ of $200 \mathrm{nM}$ Cortisol 120

4.12 (a) Electrochemical response studies of EA/Anti-Cab/DTSP/Au immunoelectrode as a function of cortisol concentration (10pM to 200nM); (b) Calibration curve between magnitude of response current and $\log$ [cortisol concentration (M)]

4.13 Percentage change in electrochemical response of the EA/Anti$\mathrm{Cab} / \mathrm{DTSP} / \mathrm{Au}$ immunoelectrode after incubation with PSA (200nM), EGFR (200nM), NSE (200nM), Cortisol (200nM) and Cortisol mixed with BSA (150nM Cortisol + 50nM BSA) .124

4.14 The standard curve obtained for determination of the cortisol concentration in saliva samples using ELISA

4.15 Comparison of cortisol concentration measured by ELISA and Electrochemical measurement on saliva samples from (a) Specimen 137 and (b) Specimen 138 at specific time intervals over three days

5.1 LTCC 32 micro-well plate fabricated for the NSA characterization .131 
5.2 Scanning electron microscope images of the DuPont 951 LTCC green tapes in the (a) Unfired and (b) Fired state

5.3 Surface roughness profile obtained for (a) unfired and (b) fired LTCC substrate using a stylus tip profilometer .134

5.4 X-Ray Diffraction pattern of DuPont 951 LTCC; (a) Fired LTCC; (b) Fired LTCC conjugated with Cortisol

5.5 (a) ELISA standard curve for cortisol concentration; (b) Picture of the 96 well micro-titer plate after completion of the ELISA assay; (c) \% NSA of $1 \mu \mathrm{M}(-\boldsymbol{-})$ ) and 500nM (- $\mathbf{\Delta - )}$ cortisol solutions incubated on LTCC as a function of incubation time

5.6 Schematic illustration of the microfluidic system for integration with an electrochemical immunosensor.

5.7 Results of CFD simulation in (a) square chamber; (b) circular chamber and (c) circular chamber with inlet and outlet channels are modified position. .141

5.8 Schematic illustration of the LTCC based microfluidic system; (b) The three-layer design of the LTCC microfluidic manifold; (c) A 3D blow out of the microfluidic chip and its integration with the sensor chip. 142

5.9 Picture of the aligning fixture with LTCC tapes being aligned prior to lamination; (b) The temperature ramping profile for the sintering process

5.10 (a) The LTCC microfluidic manifold; (b) Cross sectional microscopic image of the LTCC microchannel. .144

5.11 (a) Schematic of the LTCC microfluidic chip used for fluid flow characterization; (b) Picture of the glass window integrated into the LTCC chip; (c) Microscopic images of the fluid flow profiles in the assay chamber. .146

5.12 (a, b) Schematic of the operation of the fluidic valve; (c) Schematic of the electronic circuit designed for the activation of the fluidic valve.

5.13 (a, b) Characterization of the valving function in the fluidic valve; (c) Picture of the fully integrated microfluidic system with fluid valve, microfluidic manifold and the biosensor chip .148 
5.14 Calibration curve obtained from the electrochemical response studies of EA/Anti-Cab/DTSP/Au immunoelectrode as a function of cortisol concentration (10pM to $200 \mathrm{nM})$ in the automated microfluidic cortisol

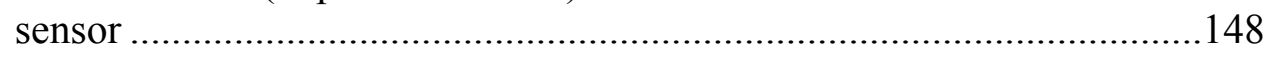

6.1 Envisioned hand-held/bench-top cortisol detection system.....................157 


\section{LIST OF TABLES}

TABLE

PAGE

2.1 Comparative table of the properties of LTCC with Silicon, PDMS and Glass.

4.1 Normal cortisol concentrations as defined by Mayo medical diagnostics 125

4.2 Cortisol values measured in saliva using the developed electrochemical immunosensor 126

4.3 Cortisol values measured in saliva samples using the commercially available cortisol ELISA kit 


\section{LIST OF ACRONYMS AND ABBREVIATIONS}

PTSD Post-Traumatic Stress Disorder

CV Cyclic Voltammetry

EIS Electrochemical Impedance Spectroscopy

SAM Self - Assembled Monolayer

DTSP Dithiobis (succinimidylpropionte)

$\mu$ IDE Interdigitated Microelectrode

LTCC Low Temperature Co-fired Ceramics

PANI Polyanaline

ELISA Enzyme Linked Immunosorbent Assay

CFD Computational Fluid Dynamics

HPA Hypothalamus-Pituitary-Adrenal

CRH Corticotrophin Releasing Hormone

ACTH Adrenocorticotrophic Hormone

ISF Interstitial Fluid

UFC Urinary Free Cortisol

CBG Corticosteroid Binding Globulin

CFI Cortisol Free Index

HPLC High Performance Liquid Chromatography

RIA Radio Immunoassay

FITC Fluorescein Isothiocyanate

ECLIA Electrochemilumenescence Immunoassay

SPR Surface Plasmon Resonance 


\begin{tabular}{ll} 
QCM & Quartz Crystal Microbalance \\
LSV & Linear Sweep Voltammetry \\
CNT & Carbon Nanotube \\
MEMS & Micro-Electromechanical Systems \\
PCR & Polymerase Chain Reaction \\
EWOD & Electrowetting-on-Dielectric \\
PDMS & Polydimethylsiloxane \\
HTCC & High Temperature Co-fired Ceramics \\
SEM & Scanning Electron Microscopy \\
EPD & Electrophoretic Deposition \\
IEP & Isoelectric Point \\
EDC & 1-ethyl-3-(3-dimethylaminopropyl) carbodiimide \\
NHS & N-Hydroxysuccinimide \\
TEM & Transmission Electron Microscopy \\
AFM & Atomic Force Microscopy \\
EDX & Energy Dispersive X-Ray Spectroscopy \\
BSA & Bovine Serum Albumin \\
PBS & Phosphate Buffer Saline \\
PSA & Prostate Specific Antigen \\
EGFR & Epidermal Growth Factor Receptor \\
NSE & Neuron Specific Enolase \\
NSA & Non-Specific Adsorption \\
POC & Point-of-Care \\
\hline
\end{tabular}




\section{CHAPTER I}

\section{Introduction}

\subsection{Motivation}

Cortisol, a steroid hormone, is a biomarker for numerous diseases and plays an important role in the regulation of various physiological processes such as blood pressure, glucose levels, and carbohydrate metabolism, within the physiological limit [1, 2]. Abnormal increase in cortisol levels inhibits inflammation, depresses immune system, increases fatty and amino acid levels in blood. While excess cortisol levels have been shown to contribute to the development of Cushing's disease with the symptoms of obesity, fatigue and bone fragility[3], decreased cortisol levels lead to Addison's disease which is manifested by weight loss, fatigue, and darkening of skin folds and scars[4]. There has been growing interest in measurement of cortisol to establish whether cortisol variation can be used as a precursor to medically and psychologically relevant events such as stress, the most recent affliction being post-traumatic stress disorder (PTSD)[57]. Since cortisol secretion is dependent on environmental and behavioral triggers, measurement of cortisol at point-of-care has become imperative to understand behavioral patterns. Furthermore, normal levels of cortisol secretion follow a circadian rhythm with cortisol levels highest during daybreak and progressively lower as the day progresses[1].

Currently, in clinical practice, total cortisol, which is the sum of free and protein bound fractions, is measured. However, free cortisol is the only biologically active fraction [8] and is responsible for all cortisol related activities in the body. Hence, in order to diagnose and properly treat cortisol-related conditions, regular estimation of free cortisol is required. Most current strategies for estimation of free cortisol are limited to 
laboratory techniques that are laborious, time-consuming, require large sample volume, expensive, and cannot be implemented at point of care [9-15]. Another significant shortcoming of the current set-up is that they only provide a snapshot of the cortisol levels of samples submitted in a diagnostic lab and do not provide a true representation of the cortisol variations that a specimen undergoes in an environment that triggers cortisol generation or suppression. Hence, real-time and continuous monitoring of cortisol levels is required to obtain valuable information that could assist doctors in better diagnosis and treatment of cortisol related conditions. Detection of 24-hour cortisol levels is currently a cumbersome process, which either involves admitting the patient for the time of study[16] or where the patient samples blood/saliva into vials at specified time intervals during the 24-hour time period, and ships it to a diagnostic laboratory[17]. The typical turnaround time is 8 to 10 days and is still not a true representation of cortisol levels in stressful environments. Hence there is a need to develop sensing strategies for the detection of cortisol for application at point-of-care. Application at point-of-care requires that the sensor be portable, have a miniaturized form factor, disposable, sterile, low power consumption, have low turnaround time and is cost effective[18-20]. The motivation for this dissertation is to explore strategies to develop cost-effective, disposable, miniaturized sensors for detection of cortisol at point-of-care. The approach is based on developing single-use, disposable nanostructured electrochemical immunosensors for the detection of cortisol and integrating it into a microfluidic system for automation of the immunosensing assay. 


\section{2 Specific Aims}

Specific Aim 1: Electrophoretically deposited Polyanaline-Ag/AgO hybrid nanocomposite based electrochemical immunosensor for mediator free detection of cortisol

The first activity that will be undertaken in this dissertation is to develop a sensing platform for the detection of cortisol. Electrochemical immunosensing in an electrochemical cell set-up will be used for the detection of cortisol in a label-free manner. To increase the electro-active surface area for higher loading of cortisol antibodies and mediator free electrochemical detection of cortisol, a nanostructured matrix of electrophoretically deposited Polyanaline (PANI)-Ag/AgO hybrid nanocomposite will be used as the matrix for immobilizing anti-cortisol antibodies on the working electrode. This task also involves the optimization of the electrophoretic deposition process and electrochemical characterization of the bio-functionalization of the electrode. Electrochemical response studies of immunoelectrode as a function of cortisol concentrations will also be undertaken to determine the sensitivity, detection limit and linear sensing range.

Specific Aim 2: Dithiobis (succinimidyl propionate) (DTSP) self-assembled monolayer (SAM) based electrochemical immunosensing platform for the detection of cortisol

Alternate to PANI-Ag/AgO as the nanostructured matrix, a self-assembled monolayer based approach will be explored, where DTSP will be utilized as the nanostructured matrix for immobilizing the Anti-cortisol antibodies onto the electrode surface. To increase the sensitivity of detection and as a step towards a point-of-care detection, microfabricated interdigitated microelectrodes ( $\mu$ IDE) will be utilized. The 
electrochemical characterization of DTSP and the electrochemical characterization of the bio-functionalization of the interdigitated microelectrodes using DTSP will be studied. Electrochemical response studies of immunoelectrode will be studied as a function of cortisol concentrations. Experimental validation of the sensor results using ELISA to determine sensor accuracy will be conducted.

\section{Specific Aim 3:}

Low Temperature Co-fired Ceramic (LTCC) based microfluidic system for integration with electrochemical immunosensor for assay automation

To automate the electrochemical immunosensing assay, a microfluidic system will be developed. As an alternative to silicon and polymer materials, the use of low temperature co-fired ceramics (LTCC) will be explored for the fabrication of a simple, cost-effective microfluidic system. To optimize the fluidic routine involved in the immunoassay, computational fluid dynamic (CFD) will be utilized to optimize the design of the microfluidic system geometry. Fabrication of the designed microfluidic system using LTCC and its integration with the electrochemical immunosensor will be conducted. System integration through addition of micro-valves, micro-pumps and microPotentiostat with the microfluidic system will be undertaken.

Non-Specific Adsorption (NSA) of Cortisol on LTCC

For the first time, a study will be conducted to determine the biocompatibility of LTCC material in terms of non-specific adsorption on cortisol on LTCC to assess the efficacy to develop LTCC based microfluidic system for immunoassay-based biosensors. 


\subsection{Outline}

In this dissertation, the development of a LTCC microfluidics based, disposable, cost effective, electrochemical immunosensing system is presented. In Chapter I, the motivation of the work and the specific aims are presented. In the first part of Chapter II, a comprehensive literature review on the physiology of cortisol and the state of the art in cortisol detection is presented. The $2^{\text {nd }}$ part of Chapter II reviews the fundamentals of microfluidics, the state of the art in microfluidic device development and a complete overview of microfluidic device fabrication using Low Temperature Co-fired Ceramics (LTCC) technology. Chapter III presents the details of the design, fabrication and testing of the immunosensing platform for cortisol detection. The synthesis of a Polyanaline$\mathrm{Ag} / \mathrm{AgO}$ hybrid nanocomposite on the working electrode for immobilization of the anticortisol antibodies and the detection of cortisol is presented. In Chapter IV, the design, synthesis and testing of the Dithiobis (succinimidyl propionate) (DTSP) self-assembled monolayer (SAM) based electrochemical immunosensing platform for the detection of cortisol is presented. Fabrication of interdigitated microelectrode is also presented in Chapter IV. Chapter V presents the design optimization, fabrication process, flow characterization and automation of the LTCC microfluidic manifold for integration with the electrochemical immunosensor chip. The summary of the research work undertaken, concluding remarks and the future prospects are discussed in Chapter VI. 


\subsection{References}

1. Nicolson, N.A., Measurement of cortisol. Handbook of physiological research methods in health psychology, 2008: p. 37-74.

2. Levine, A., et al., Measuring cortisol in human psychobiological studies. Physiology \& Behavior, 2007. 90(1): p. 43-53.

3. McEwen, B.S., Cortisol, Cushing's Syndrome, and a Shrinking Brain-New Evidence for Reversibility. Journal of Clinical Endocrinology \& Metabolism, 2002. 87(5): p. 1947-1948.

4. Edwards, O., et al., Changes in Cortisol Metabolism Following Rifampicin Therapy. The Lancet, 1974. 304(7880): p. 549-551.

5. Yehuda, R., S.L. Halligan, and R. Grossman, Childhood trauma and risk for PTSD: Relationship to intergenerational effects of trauma, parental PTSD, and cortisol excretion. Development and Psychopathology, 2001. 13(03): p. 733-753.

6. Delahanty, D.L., A.J. Raimonde, and E. Spoonster, Initial posttraumatic urinary cortisol levels predict subsequent PTSD symptoms in motor vehicle accident victims. Biological Psychiatry, 2000. 48(9): p. 940-947.

7. Yehuda, R., S.L. Halligan, and L.M. Bierer, Cortisol levels in adult offspring of Holocaust survivors: relation to PTSD symptom severity in the parent and child. Psychoneuroendocrinology, 2002. 27(1): p. 171-180.

8. le Roux, C.W., et al., Free cortisol index is better than serum total cortisol in determining hypothalamic-pituitary-adrenal status in patients undergoing surgery. Journal of Clinical Endocrinology \& Metabolism, 2003. 88(5): p. 20452048.

9. Tilden, R., New, advantageous approach to the direct radioimmunoassay of cortisol. Clinical chemistry, 1977. 23(2): p. 211-215.

10. Ruder, H.J., R.L. Guy, and M.B. Lipsett, A radioimmunoassay for cortisol in plasma and urine. Journal of Clinical Endocrinology \& Metabolism, 1972. 35(2): p. 219-224.

11. Turpeinen, U., et al., Determination of urinary free cortisol by HPLC. Clinical chemistry, 1997. 43(8): p. 1386-1391.

12. Yaneva, M., G. Kirilov, and S. Zacharieva, Midnight salivary cortisol, measured by highly sensitive electrochemiluminescence immunoassay, for the diagnosis of Cushing's syndrome. Central European Journal of Medicine, 2009. 4(1): p. 59-64. 
13. Lewis, J. and P. Elder, An enzyme-linked immunosorbent assay (ELISA) for plasma cortisol. Journal of steroid biochemistry, 1985. 22(5): p. 673-676.

14. Frasconi, M., et al., Surface plasmon resonance immunosensor for cortisol and cortisone determination. Analytical and Bioanalytical Chemistry, 2009. 394(8): p. 2151-2159.

15. Yang, X.-D., W.-B. Chang, and Y.-X. Ci, Time-resolved fluorescence immunoassay with measurement of a europium chelate in solution: dissociation conditions and application for determination of cortisol. Analytical Chemistry, 1994. 66(15): p. 2590-2594.

16. Czeisler, C.A., et al., Episodic 24-hour cortisol secretory patterns in patients awaiting elective cardiac surgery. Journal of Clinical Endocrinology \& Metabolism, 1976. 42(2): p. 273-283.

17. Brezina, P.R., E. Haberl, and E. Wallach, At home testing: optimizing management for the infertility physician. Fertility and Sterility, 2011. 95(6): p. 1867-1878.

18. Ahn, C.H., et al., Disposable smart lab on a chip for point-of-care clinical diagnostics. Proceedings of the IEEE, 2004. 92(1): p. 154-173.

19. Wang, J., Electrochemical biosensors: towards point-of-care cancer diagnostics. Biosensors and Bioelectronics, 2006. 21(10): p. 1887-1892.

20. Soper, S.A., et al., Point-of-care biosensor systems for cancer diagnostics/prognostics. Biosensors and Bioelectronics, 2006. 21(10): p. 19321942. 


\section{CHAPTER II}

\section{Literature Review}

\section{1 Cortisol}

Cortisol is a one of the important glucocorticoids (a family of steroid hormones) produced by the adrenal glands. Cortisol is small molecular weight (362.46 grams/mole) hormone, given by the chemical formula (11ß)-11,17,21-trihydroxypregn-4-ene-3,20dione. Fig. 1 presents the chemical structure and the 3D arrangement of the atoms.

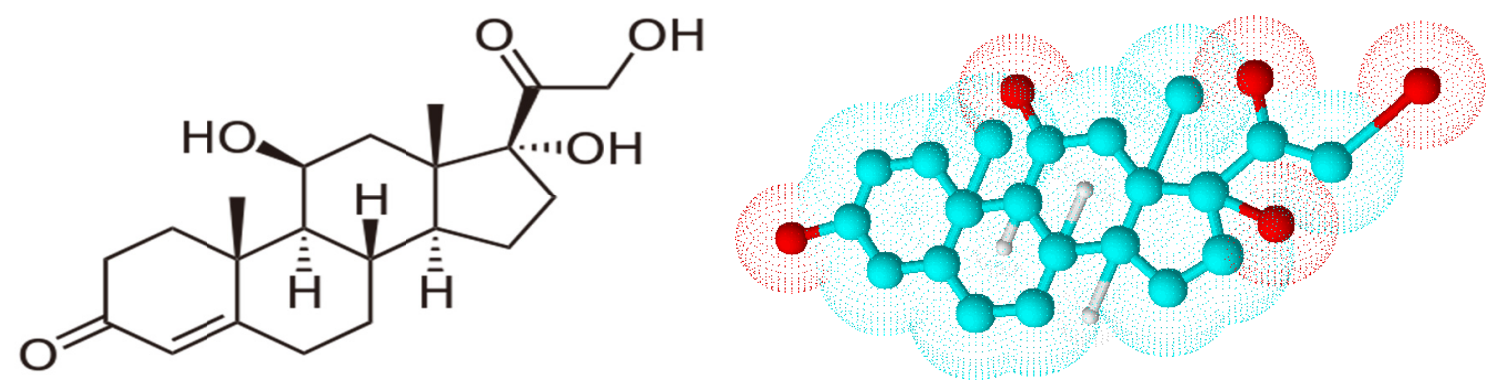

Fig 2.1 Chemical structure of cortisol; (a) 2D and (b) 3D

Cortisol plays multiple roles in human physiology, including maintaining blood glucose levels, blood pressure regulation, and homeostasis of the cardiovascular, immune, skeletal and endocrine system. Cortisol levels fluctuate through a 24 hour cycle called the circadian rhythm (Fig. 2), highest values 30 minutes after awakening and gradually tapers down to a minimum during night sleep[1]. Other controllable factors also affect cortisol levels such as eating patterns and physical activity. The most dominating effect on cortisol variation comes from psychological/emotional stress, which is why cortisol is popularly called the "Stress-hormone"[2]. 


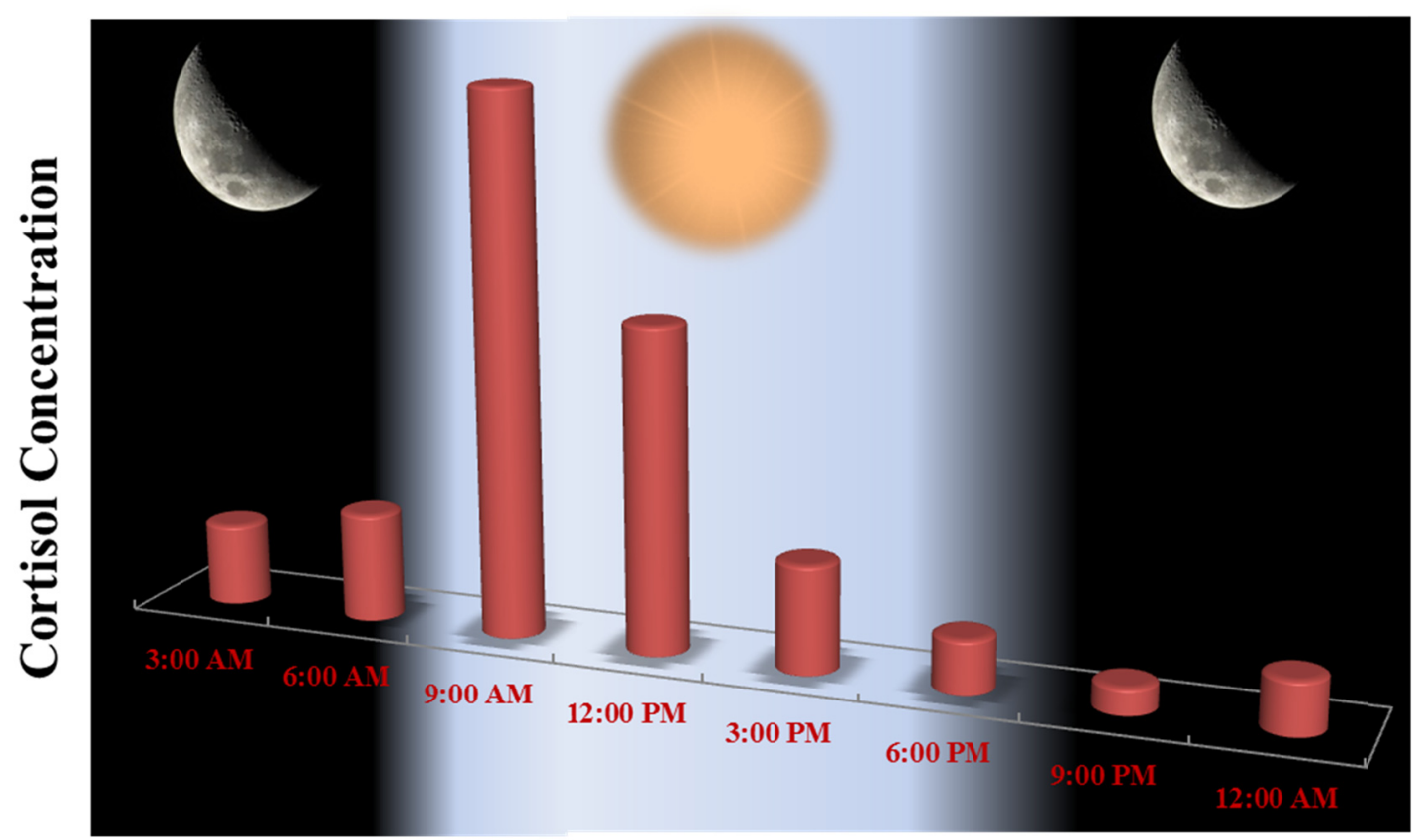

Time of Day

Fig 2.2 The typical diurnal variation of cortisol levels over a 24 hour cycle.

The effects of abnormal cortisol variations for prolonged periods of time reflect in the disruption of the homeostasis in physiological processes. Cushing's syndrome is a typical example of hyper-cortisol secretion, where the affected individual experiences sever weight gain, suppressed immune system, osteoporosis, diabetes mellitus and several other negative effects[3]. Addison's disease, which is a condition arising from hypo-cortisolism, results in low blood pressures, hypoglycemia, convulsions and prolonged fever[4].

\subsubsection{Secretion of Cortisol}

Cortisol is a hormone that is secreted from the adrenal glands located above the kidneys. Cortisol is the end product of the Hypothalamic-Pituitary-Adrenal (HPA) axis, which is the main component of the human body's adaptive system to maintain regulated 
physiological processes under changing environmental factors. As the name suggests, the HPA axis is a complex signaling system between the hypothalamus in the brain, the pituitary glands and the adrenal glands. Fig. 3 presents a schematic of the HPA axis in which a typical response to an environmental trigger is initiated by the hypothalamus that releases a hormone called the $\mathrm{CRH}$ (Corticotrophin Releasing Hormone) that travels to the pituitary glands. Specialized cells that work synergistically with the pituitary glands, release ACTH (Adrenocorticotrophic Hormone) into the blood stream that travels to the adrenal cortex. The adrenal cortex responds by increasing the production of cortisol. Since the adrenal glands have no visible innervation, it can be inferred that ACTH is the sole stimulant for initiating cortisol production. The adrenal glands do not store cortisol, but they are prevalent in the form of precursors. Cholesterol undergoes multiple catalyzed oxidation reactions to result in the formation of cortisol. This entire process takes place in a time space of few minutes. The HPA axis is a negative feedback system, where Cortisol plays a critical rule in the homeostasis of the HPA axis. Moderate homeostatic alterations in the HPA axis is beneficial for the physiological and psychological development of the human body, sustained and prolonged exposure to environmental triggers such as stress leads to abnormal levels of cortisol in the circulatory system. 


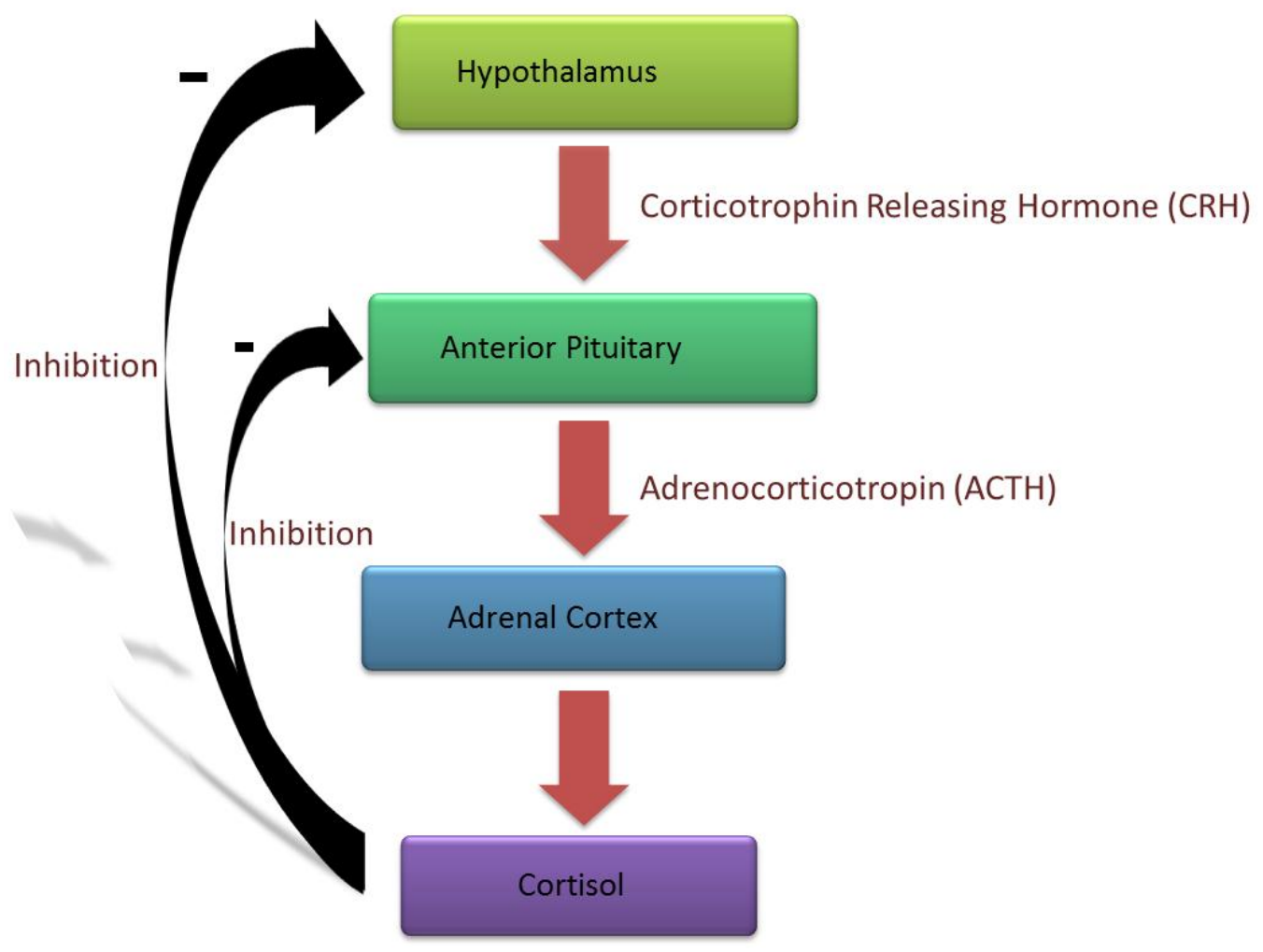

Fig 2.3 Cortisol secretion regulated by the HPA axis

\subsubsection{Source of Sample}

Secreted cortisol finds its way into the circulatory system and can be found in detectable quantities in several biofluids. In this section, an assessment of the advantages and disadvantages of using various biofluids such as urine, blood, sweat, interstitial fluid (ISF) and saliva for the detection of cortisol is presented.

\subsubsection{Urine}

Cortisol level in urine is measured over a 24-hour period and is referred to as the 24-hour Urinary free cortisol (UFC) test. Only free cortisol, which is the active form of 
cortisol in the human body, is found in urine and is hence a relevant biofluids for the detection of cortisol. The excretion of hormones, salts and other waste chemicals through urine is a well-characterized process with good knowledge about the concentration of these waste substances under normal function. Observation of drastic variation in concentrations of hormones such as cortisol can be used to diagnose abnormalities in the adrenal function. Measurement of cortisol in urine typically requires the collection of all the urine generated over a 24 hour time period. Normal range for cortisol in urine is 10 to $100 \mu \mathrm{g} / 24 \mathrm{hrs}$. A review of the reported literature for urinary cortisol assays has been presented by Brossaud et. Al. [5]

While the 24-hour urinary free cortisol test provides a means for a non-invasive, painless method of obtaining body fluid for cortisol measurement, it also poses several drawbacks with respect to convenience and reliability. Since the collection of the urine is spanned over a 24 hour time period, sample collection becomes an inconvenient process where the patient needs to carry the special urine collection container all day long or has to remain confined to a location for the 24 -hour period. The container also needs to be stored under refrigeration from the time of collection till it is delivered to a diagnostic lab for testing. Also, several factors such as pregnancy and medication such as diuretics can alter the concentration of cortisol in urine making it a lesser reliable biofluids for cortisol detection. These are several additional factors have severely limited the use of urine to cases where patients are admitted to the hospital for long-term treatments. The requirement of 24 hour sample collection has rendered urine unfit for real time detection at point of care. 


\subsubsection{Interstitial Fluid (ISF)}

ISF is an extra cellular fluid that surrounds the cells in the human body. In composition, it is similar to blood plasma. Metabolites and proteins move into ISF as they move from capillaries to cells. In general, small to moderate sized molecules, including glucose, ethanol, and cortisol are found in ISF in similar proportion as in blood. Thus, periodic calibration using blood sampling is not required to obtain the concentration of these metabolites from ISF. ISF is present just below the skin, but the low permeability of the epidermal keratinized layer (the stratum corneum) blocks the permeation of the fluid through the skin. However, obtaining ISF for detection of a target biomarker could require an invasive approach as it is not readily accessible. Several approaches have been reported in literature to obtain ISF in a minimally invasive, painless process. Venugopal et. Al. [6] have reported the construction of an ISF harvesting system that utilizes a lowenergy laser to create micropores in the stratum corneum (the uppermost layer of dead cells) (Fig. 4). The diameter of the micropores is approximately equal to that of a human hair. The micropores only penetrate the stratum corneum and, hence, this procedure is essentially painless. ISF is drawn through these micropores continuously by application of a small amount of vacuum pressure. Harvesting of ISF using this set-up is reported at a rate of $10 \mu \mathrm{l} / \mathrm{hr}$. 

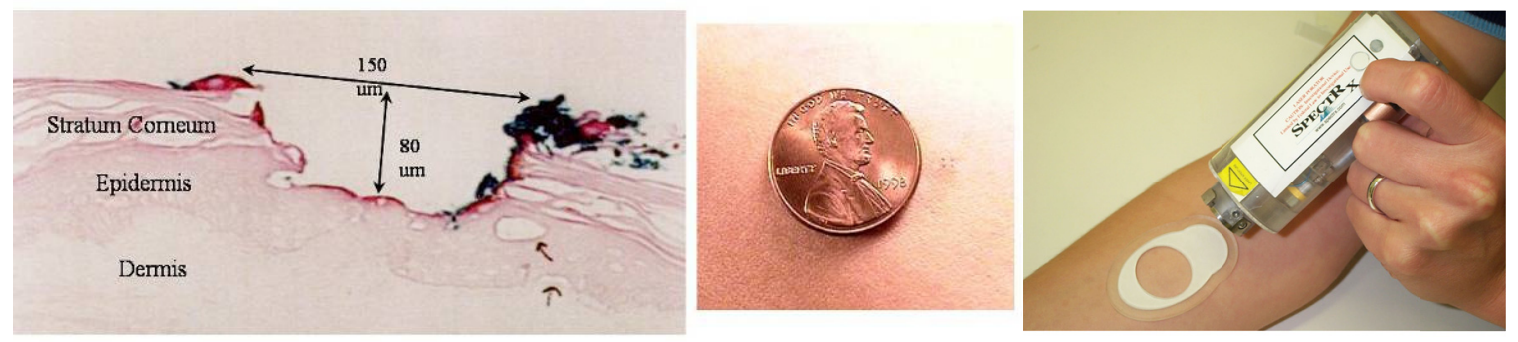

Fig 2.4 (a) Cross-section of micropore generated through laser ablation; (b) Four pores relative to the size of a penny; (c) Handheld laser source used to create micropores.

Coupled with an electrochemical detection system, Bhansali et. Al. [7] have reported the detection of cortisol using the same micro poration set-up. Cortisol levels in ISF were found to be $3-4$ times larger than that in saliva, which makes ISF an attractive biofluid for detection of cortisol. While this set-up may provide a means to access ISF for cortisol detection, the low harvesting rate $(10 \mu \mathrm{l} / \mathrm{hr})$ would limit its applicability for obtaining instantaneous cortisol values in a point of care setting.

Micromachined microneedles for transdermal application are another option to harvest ISF[8,9]. Microneedles have been used extensively for transdermal drug delivery $[9,10]$. Smith et. al. [11] have reported the design, fabrication and testing of a hollow microneedle array containing fluidic microchannels for the transdermal extraction of ISF from human skin. Wang et al.[12] have also reported the fabrication of glass microneedles for ISF extraction for glucose monitoring. These approaches show promise for creating painless, minimally invasive methods for extraction of ISF. Microneedles based transdermal ISF extraction may find good application in wearable biosensing system, where there is a critical need to continuously sample body fluids such as ISF at a low sampling rate. However, concerns regarding biocompatibility and biodegradation of 
the microneedles, protection from infection due to usage of needles and other sterility issues will need to be carefully addressed for successful implementation.

\subsubsection{Hair}

The use of hair as the biological sample for analysis and testing in forensic sciences, toxological science, doping control and clinical diagnostics has gained considerable attention over the last two decades [13]. Human hair grows at a predictable rate of approximately $1 \mathrm{~cm} /$ month. Cortisol is known to deposit in the shaft of the hair and the most proximal $1 \mathrm{~cm}$ segment of hair closest to the scalp approximates the last month's cortisol production and so on. The mechanism for the incorporation of cortisol into the hair shaft has been proposed by Bennett et. Al. [13]. Fig. 5 presents an illustration of cortisol storage mechanism in the hair follicle and its usage for analysis of long-term exposure of cortisol. Cortisol is thought to enter hair primarily at the level of the medulla of the hair shaft via passive diffusion from blood. In this scenario hair cortisol would be hypothesized to reflect the integrated free cortisol fraction rather than the total cortisol concentration in serum. 


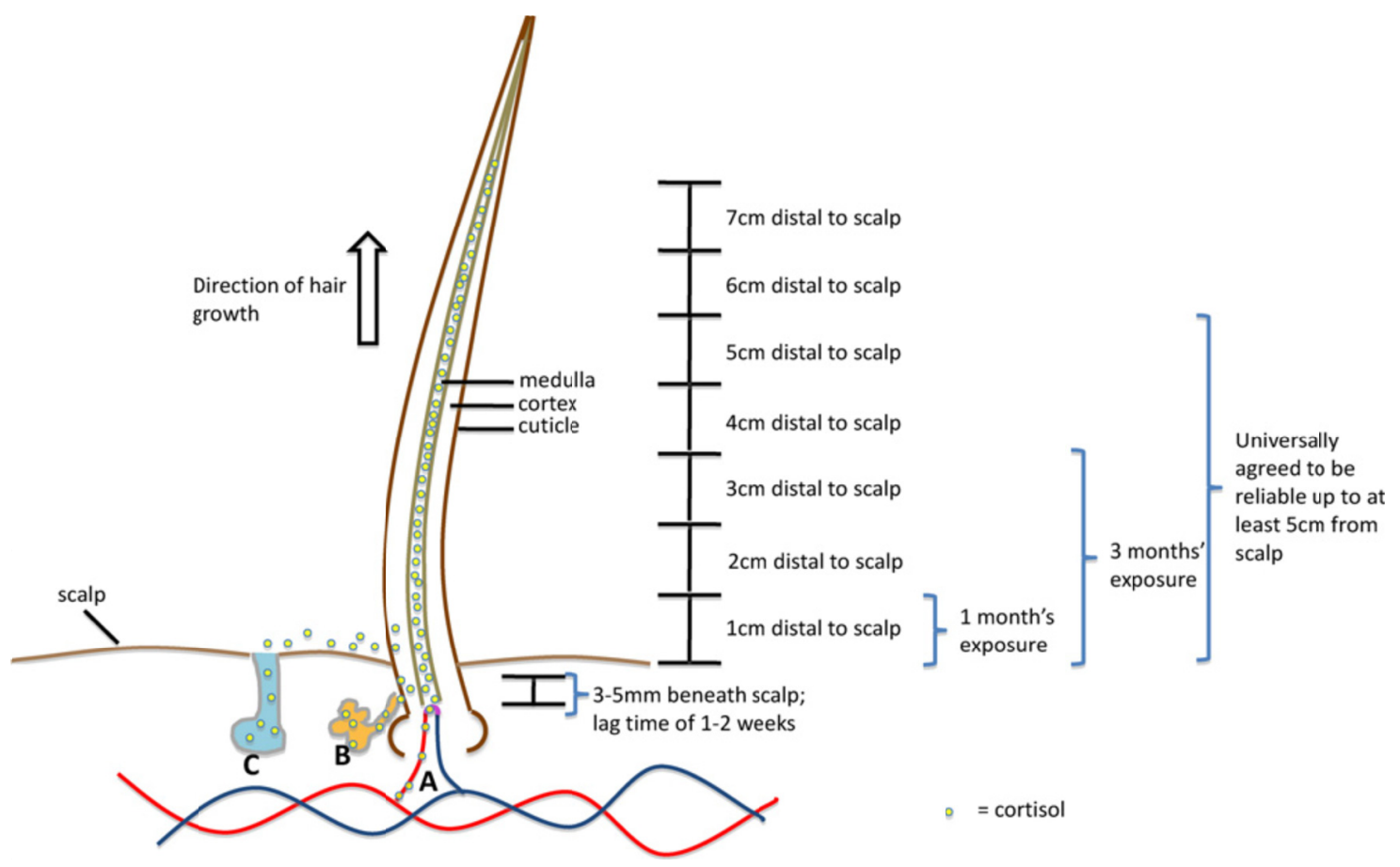

Fig 2.5 Schematic illustration of the accumulation of cortisol in the medulla of a hair follicle.

One of the first studies to establish feasibility of using hair for cortisol detection was reported by Koren et al.[14] using hair from wild hyraxes. Detection was performed using a modified salivary ELISA protocol. Sauve et al. [15] reported the first study on human hair samples for cortisol detection and reported a reference range from 1.7 to $153.2 \mathrm{pg} / \mathrm{mg}$. The obtained values were compared to those obtained from saliva, serum and 24-hour urine. A positive correlation was identified only to that of 24-hour urine, with no correlation to that of saliva or serum. Hair cortisol measurement surely provides a non-invasive method of obtaining a biological sample. Since the cortisol concentration in hair is hypothesized to represent long-term system exposure, it could be used as indexing method to maintain a cortisol secretion calendar. From the various other reported studies [16-22] for cortisol detection from human hair, it is evident that cortisol 
values only for long-term exposure to factors such as stress can be obtained. The resolution of the data obtained is in the order of months, with very little clinical data available to support the correlation of hair cortisol levels to stress levels.

\subsubsection{Sweat}

Sweat analysis is a well-established method for diagnosis certain conditions such as cystic fibrosis and drug abuse. Sweat patches could be used as effective non-invasive tools to collect biofluids[23]. Cortisol in sweat has been measured by Russell et al[24] with cortisol concentrations ranging from $141.7 \mathrm{ng} / \mathrm{mL}$ (daytime) to $8.16 \mathrm{ng} / \mathrm{mL}$. It is hypothesized that there exists a strong correlation between cortisol levels in sweat and hair due the path that cortisol traverses from serum, sebum and sweat to the hair. However, very little is known about cortisol in sweat and several inherent drawbacks exist in obtaining reliable and repeatable sweat samples to accurately measure cortisol in sweat. Perspiration is dependent on several factors such as weather conditions (humidity, temperature), geographic conditions, physical activity levels, ambient temperature or conditioned temperature and genetic make of the patient.

\subsubsection{Blood}

More than $90 \%$ of cortisol in blood is in an inactive state being bound to corticosteroid binding globulin (CBG) and serum albumin. Only $10 \%$ of total cortisol is in a biologically active state to participate in cortisol initiated processes. Typically assays for measuring cortisol in blood involves measuring the total cortisol (bound + free) and then the active fraction, called the Cortisol Free Index (CFI) is deduced using the Coolen's 
equation[25]. Blood sampling for cortisol detection has been the oldest form of biofluid sampling. The nominal value for cortisol in blood varies from $25 \mu \mathrm{g} / \mathrm{dL}$ (9AM) to $2 \mu \mathrm{g} / \mathrm{dL}$ (midnight). Blood however is afflicted by many drawbacks, which has made it a last choice sampling fluid. Sampling blood requires attention from medical staff and specialized, sterile equipment with an ever-existing concern for infections. While cortisol is a stable molecule at room temperature, its presence in plasma requires special handling and storage condition as it is considered a biohazard. Since sampling blood requires puncture of veins, a painful procedure, the stress experienced by patients prior to and during sampling may elevate cortisol levels[26]. Although the typical response time for cortisol spiking is 10 to 15 minutes in humans, prior knowledge of venipuncture can initiate the stress response induced cortisol spiking. Also, the costs associated with staff, equipment, handling and storage makes blood based assay a shunned option.

\subsubsection{Saliva}

Over the last few years, saliva has gained considerable attention as a biofluid for analysis and detection of cortisol concentrations. This has come about mainly due to inherent advantages associated with saliva. First and foremost, a well-documented and studied strong correlation exists between salivary and blood cortisol levels[27, 28]. Also, of high importance is the fact that cortisol in saliva exists entirely in the free state in saliva unlike in blood ( $90 \%$ bound), resulting in detection of the relevant (biologically active) form of cortisol. This is mainly due to the filtering of the CBG and Albumin bound cortisol during capillary exchange and other intracellular mechanisms at the salivary ducts. Harvesting samples for analysis is almost completely non-invasive with little or no 
discomfort to the specimen providing the sample[29]. The last few years has seen the establishment of standard operation procedures for collection of saliva, which has led to lesser variability in analyzed results. The samples can be harvested with minimum efforts that have facilitated patients to collect their own samples at home. Cortisol remains stable in saliva at room temperature, resulting in reduced costs and efforts in sample handling and storage. All these advantages of salivary techniques have led to saliva being on the verge of becoming the preferred source of body fluid for detection of cortisol. However, there are certain aspects that may adversely affect the prospects of saliva based cortisol detection. Since only the active component of cortisol (free cortisol) is present in saliva, the concentration of cortisol is much lower than that of blood. The nominal values for cortisol in saliva during the diurnal cycle varies from $0.5 \mu \mathrm{g} / \mathrm{dL}$ to $0.05 \mu \mathrm{g} / \mathrm{dL}$, which requires high sensitivity assays with low detection limits for efficient detection of cortisol concentration in saliva. A strict compliance to the standard operating procedure for saliva collection can lead to erroneous results. Sometimes, presence of blood due to oral leasons may lead to elevated levels of cortisol and cause erroneous results. Salivary cortisol assays have been reported in literature extensively for characterizing the circadian rhythm[30], Cushing's syndrome[31], Addison's disease[32], adrenal abnormalities[33] and stress related disorders[34].

The overwhelming advantages of using saliva as a biofluid for biomarker detection has made it the preferred method. Also, the ease of sample collection, handling and storage has heightened the prospects of applying point of care sensors for real-time and continuous detection of cortisol. For these reasons, this research undertakes the development and characterization of salivary assays for electrochemical immunosensing 
of cortisol and its application of point-of-care through incorporating into an automated microfluidic system.

\subsubsection{Detection of Cortisol: State of the art}

Having studied the physiology of cortisol and evaluating the pros and cons of the different sources of biofluids for cortisol detection, this section provides a comprehensive overview of the various detection techniques for quantification of cortisol.

\subsubsection{High Performance Liquid Chromatography (HPLC)}

HPLC is the older and one of the first techniques to detect cortisol. HPLC is a chromatographic technique that used high pressure (50 to 350 bar) to separate a mixture of compounds using a process of mass transfer induced adsorption. While RIA was used to determine cortisol in serum, HPLC was used to determine cortisol concentrations in saliva. HPLC lacks the specificity that is required in measuring low concentrations, as the technique is severely affected by interference from co-eluting substances in the sample. HPLC also requires many preprocessing procedures such as solid-phase extraction[35].

\subsubsection{Immunoassays}

Immunoassays are based on the ability of an antibody to recognize and selectively bind to an antigen, referred to as the analyte. The high degree of selectivity and specificity of an antigen-antibody binding makes immunoassays the gold standard technique for detection of presence, and measurement of the concentration of the analyte of interest. After successfully capturing the analyte, traditional process of immunoassays employ a label to 
detect the analyte. Enzymes, fluorescent tags and radioisotopes are some of the commonly used labels. Immunoassays may be performed in different configurations, depending on the analyte, label and detection technique. The primary classification of immunoassays is based on washing (heterogeneous) and no-washing (homogenous) of the sensing substrate after sample incubation. While homogenous immunoassays involve lesser assay steps, its application is limited by sensitivity and noise arising from nonspecific interactions. Heterogeneous immunoassays are further classified based on competitive/noncompetitive and labeled/unlabeled binding of the antigen to the antibody (Fig. 6). In a competitive immunoassay, the analyte of interest (unlabeled) competes with a known concentration of labeled analyte of the same kind. At the completion of the reaction, the concentration of the unbound, labeled analyte is measured, from which the concentration of the analyte of interest is deduced (Fig. 6a). Accordingly, in a noncompetitive immunoassay, only the analyte of interest is allowed to freely bind to the antibodies and a then the analyte is labeled to measure the concentration (Fig. 6b). To increase the sensitivity and selectivity of the immunoassay, a two-site or sandwich immunoassay is performed, where two a pair of antibodies are used, one for capturing the analyte and the other for detection (Fig. 6c). 


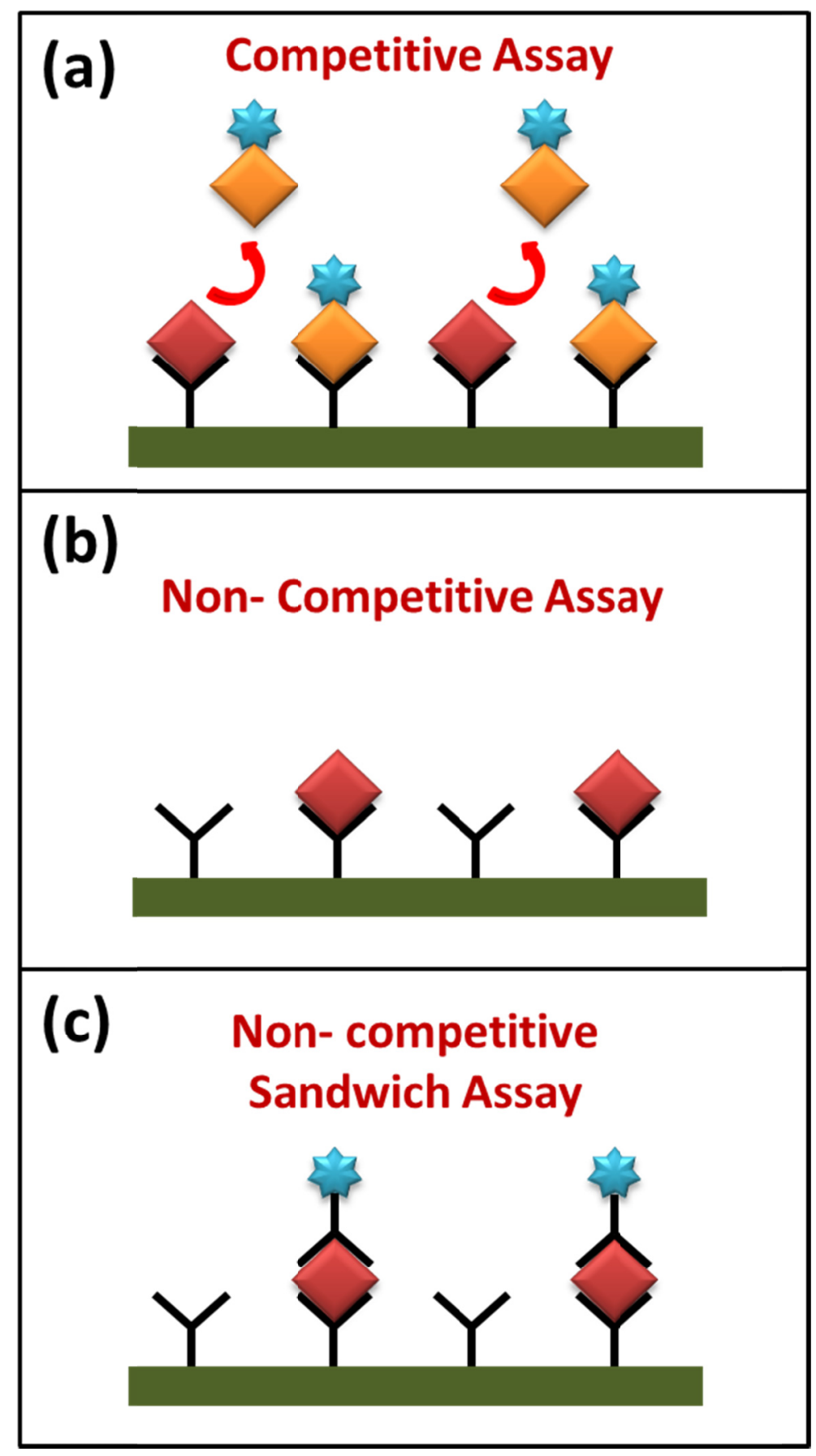

Fig 2.6 Schematic illustrations of various immunoassay techniques

Several transduction techniques have been developed to obtain a measurable signal that is proportional to the concentration of the captured analyte and can be broadly classified as optical and electrical. For detection of cortisol, radioimmunoassay (RIA), 
which involves the use of radioisotopes as label was reported in the late 1970's [36-40]. Since the 1980's use of RIA has diminished due to the potential harmful effects of handling radioisotopes. The focus has shifted towards the use of labels with fluorescing property. The use of fluorescent tags such as fluorescein isothiocyanate (FITC) [41], mixture of sulfuric acid and acetic acid[42] have been used as the label in cortisol immunoassays. A fluorescence detector is utilized to read the intensity of fluorescence, which is proportional to the concentration of the fluorescence labeled analyte. Another popular optical based immunoassay is the electrochemilumenescence immunoassay (ECLIA). ECLIA is based on the electrogenerated chemilumenescing property of intermediates undergoing a highly exergonic reaction to produce an electronically excited state that emits light. ECLIA has developed into highly reliable immunoassay technique due to the high sensitivity and precise control over the electrochemical reaction. ECLIA has found application in many clinical studies for cortisol detection in human samples for Cushing's syndrome[43, 44], obesity[45], athletic disorders [46] and stress related disorders such as PTSD[47, 48]. Of all the available labeled immunoassays, Enzyme Linked Immunosorbent Assay (ELISA) is the most sensitive and versatile and is today considered the gold standard in protein concentration determination. ELISA is typically performed in a sandwich format, where an enzymatic substrate is added to the secondary antibody to amplify the colorimetric or fluorescent signal, thereby providing a high sensitivity. Detection of cortisol using ELISA is used widely $[49,50]$ has often been the technique used to validate results obtained from newer techniques being developed for cortisol detection [51]. ELISA kits for detection of various analytes are commercially available now. While this method is the most widely used technique in research labs and 
industry, the method is limited by the need for large sample and reagent volumes and complexity arising from multiple assay steps and large incubation times.

To overcome the tedious processes, cost and shortcomings of the assay performance, focus has shifted towards developing label-free immunosensing techniques with high sensitivity, lower detection limits and broader detection range[52]. One such technique that demonstrates label-free detection is based on Surface Plasma Resonance (SPR)[53]. SPR works on the principle of oscillation of valence electrons in a conducting substrate irradiated with light. SPR is highly sensitive to adsorption of molecules onto the substrate, where the resonance curves shift to higher angles with adsorption of molecules onto the surface. SPR has shown promise as a method to quantitatively measure the capture of analyte on substrates coated with anti-bodies. The associated detection optics and electronic for SPR measurement can be reduced to miniaturized form factors and has hence attracted efforts to create point-of-care immunosensors[54]. More recently, detection of cortisol in saliva[55] and other biofluids using SPR has been reported in literature. On the same lines as SPR, another technique gaining ground for immunosensing is based on the resonance property of quartz crystal microbalance $(\mathrm{QCM})[56]$.

\subsubsection{Electrochemical Immunosensing}

Since the focus of this dissertation is on developing strategies for cortisol detection based on electrochemical immunosensing, this topic is discussed elaborately. Among the label-free technologies for detection in immunosensing, electrochemical immunosensing has emerged as the most promising alternative to optical detection. 
Electrochemical immunosensing is based on the principle of measuring the changes in electrical properties of a conductive material due to the adsorption of an analyte on the surface functionalized with antibodies. The electrical change is attributed to the change in the concentration of the electro active redox species at the electrode proximity. The mature processing capability of the microelectronics industry has allowed building microelectrodes that provide high sensitivity and very low detection limits. The simplicity of electronic circuitry for electrochemical detection and cheap volume manufacturing has driven efforts to bring electrochemical immunosensing up to speed with other immunosensing techniques. The elementary requirement for an electrochemical immunosensor is a conductive substrate, which permits the immobilization of antibodies. Metals such as $\mathrm{Au}, \mathrm{Ag}$ and Platinum and carbon electrodes intrinsically allow adsorption of antibodies, but the resulting binding is not robust and stable enough. To overcome this problem and to improve the electrochemical response, nanomaterials are employed as a linker molecule between the electrode surface and the antibodies. The choice of material is dependent upon the formation of bond with the conducting electrode surface and the presence of functional groups on the nanomaterial for covalent binding to antibodies. A detailed discussion on nanomaterial's for electrochemical biosensing is presented in a later part in this chapter.

Many strategies for sensing the immuno-reaction exist and is represented in Fig. 7. The traditional sandwich immunoassay may be utilized, in which, the secondary antibody is an antibody-enzyme conjugate, and the added enzyme substrate produces an electro active product that can be measured at the electrode surface (Fig. 7a). A simpler and efficient strategy, called the direct assay, involves measurement of the change in 
electro activity of the electrode due to reduced electro active probes arising from the spatial blocking of the electrode surface area by the adsorption of the captured analyte (Fig. 7b). This technique, though simple, may sometimes lead to false positive results due to non-specific adsorption and hence effective chemical strategies need to be employed to overcome non-specific adsorption.

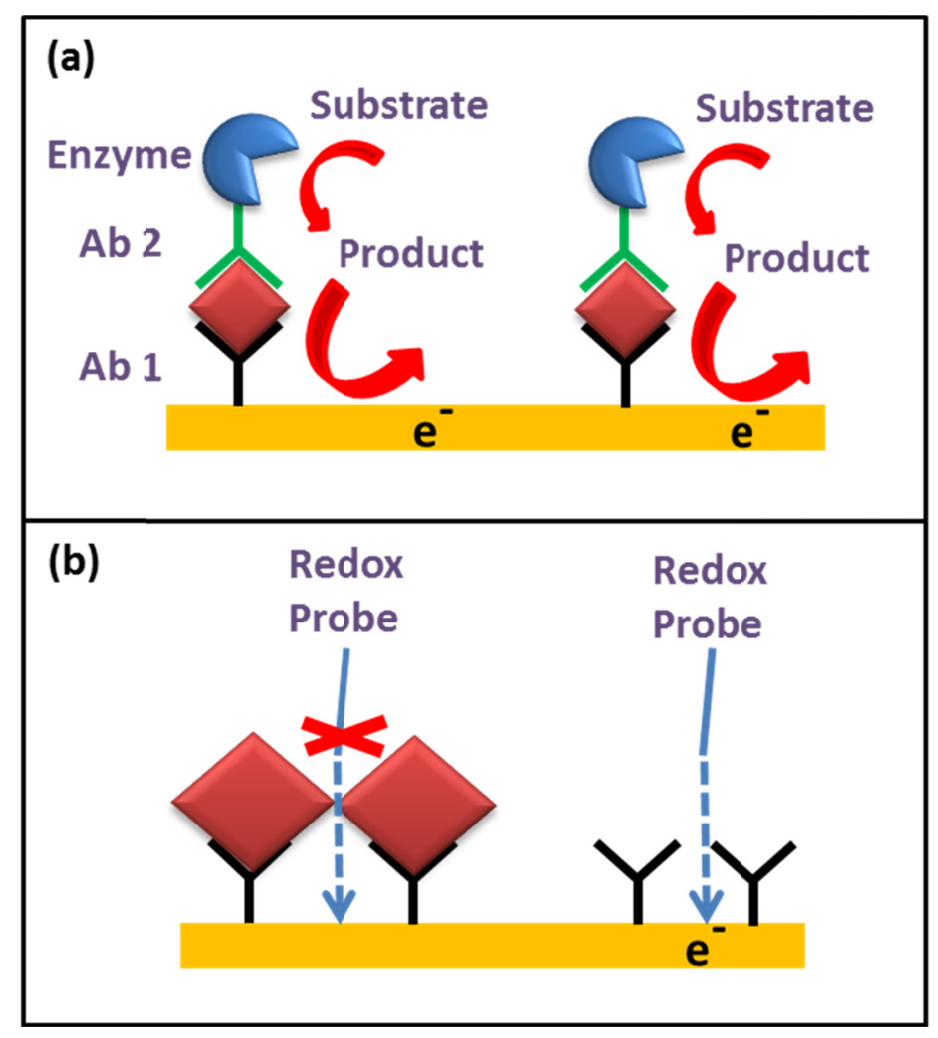

Fig. 2. 7 Schematic of strategies used for electrochemical immunosensing

All electrochemical reactions are governed at least in part by the Nernst equation, which fundamentally defines the relationship between the potential at an electrode and the concentrations of two redox species (oxidized and reduced) involved in a redox reaction. The redox reaction and the Nernst equation are represented as:

$$
\mathrm{O}+(n) \mathrm{e}^{-} \leftrightarrow \mathrm{R}
$$




$$
E=E^{o}+\frac{R T}{n F} \ln \left(\frac{C_{o}}{C_{R}}\right)
$$

$E^{o}$ is the redox potential for the coupling involved in the oxidation and reduction, $C_{O}$ is the concentration of the oxidized species, $C_{R}$ is the concentration of the reduced species, $R$ is the universal gas constant $\left(8.3144 \mathrm{~J} \mathrm{~K}^{-1} \mathrm{~mol}^{-1}\right), T$ is the absolute temperature, $n$ is number of moles of electrons transferred in the cell reaction, $F$ is Faraday constant $\left(9.648 \times 10^{4} \mathrm{C} \mathrm{mol}^{-1}\right)$.

Based on the electrical property being measured, electrochemical sensors can be classified as Potentiometric, Voltammetric and Impedimetric. Potentiometric immunosensors passively measure the potential between two electrodes. The set-up consists of an electrode pair, where the potential on one electrode is maintained constant (reference) and the potential at the other electrode (immunoelectrode) is measured with respect to the reference electrode. The immunoelectrode usually uses electrodes that have been made ion selective. The measured potential can then be correlated to the concentration of the analyte directly from the Nernst equation. Potentiometric immunosensing is not a popular strategy due lack of sensitivity, accuracy, precision and stability[57]. This is due to a basic assumption that the measured potential accurately reflects the equilibrium position of an electrochemical reaction, which is often not the case. The interference arising from the sample matrix will supersede the signal arising the specific binding of an analyte. Voltammetric techniques involve the application of a constant potential to the immunoelectrode surface and measuring the resulting steady state current generated by the electroactive redox species. It may be noted that the difference between voltammetry and amperommetry is trivial and confusion in literature 
is due to the introduction of scanning techniques in voltammetry, where the potential is help for a brief length of time (for current measurement) and then increased/decreased for further measurements. Voltammetric techniques can be described as a function of Voltage, Current and Time. Linear sweep voltammetry (LSV), the potential at the working electrode (immunoelectrode) is swept at a specific rate (volts/sec) from a lower potential to a higher potential, and the current at each potential step is measured[58]. A variant to this technique, called cyclic voltammetry (CV) entails applying a triangle potential waveform from an initial value to a predetermined upper limit, where the direction of the sweep is reversed[59]. As the potential is swept back and forth, the current on the working electrode is observed. Analysis of the current response can be used to study the thermodynamics and kinetics of electron transfer at the electrodesolution interface. Moreover it helps to study the kinetics and mechanism of solution chemical reactions initiated by the heterogeneous electron transfer. Cyclic voltammetry can also be described as an analytical tool by which information about the analyte can be obtained by measuring the current flowing on the working electrode that either oxidizes or reduces the analyte. The magnitude of this current is proportional to the concentration of the analyte in solution, which allows cyclic voltammetry to be used in determination of the analyte concentration. The current at the working electrode (faradaic current) can be plotted as a function of time or since the potential is linearly related with time, as a function of voltage. The representation of the response in cyclic voltammetry experiment is usually the current-potential curve, which is called "cyclic voltammogram" (Fig. 8).

As the potential is swept in the forward direction, a cathodic peak is observed. Reduction occurs in this positive scan and the current resulting from the reduction is 
called cathodic peak current. The potential value in this point is called as cathodic peak potential. The representations of these terms are $i_{p c}$ and $E_{p c}$ respectively. Note that the reduction current is taken as positive as the cathodic sweep goes from left to right. At the switching potential, the direction of the potential sweep is reversed. In the reverse scan, oxidation occurs and a peak resulting from the oxidation process is called anodic peak current. A negative anodic peak current is observed in the case of oxidation process. The representations for the anodic peak current and anodic peak potential are $i_{p a}$ and $E_{p a}$ respectively. A typical cyclic voltammogram is given in Fig. 8 with a clear representation of the defined characteristics such as $i_{p c}, E_{p c}, i_{p a}$ and $E_{p a}$.

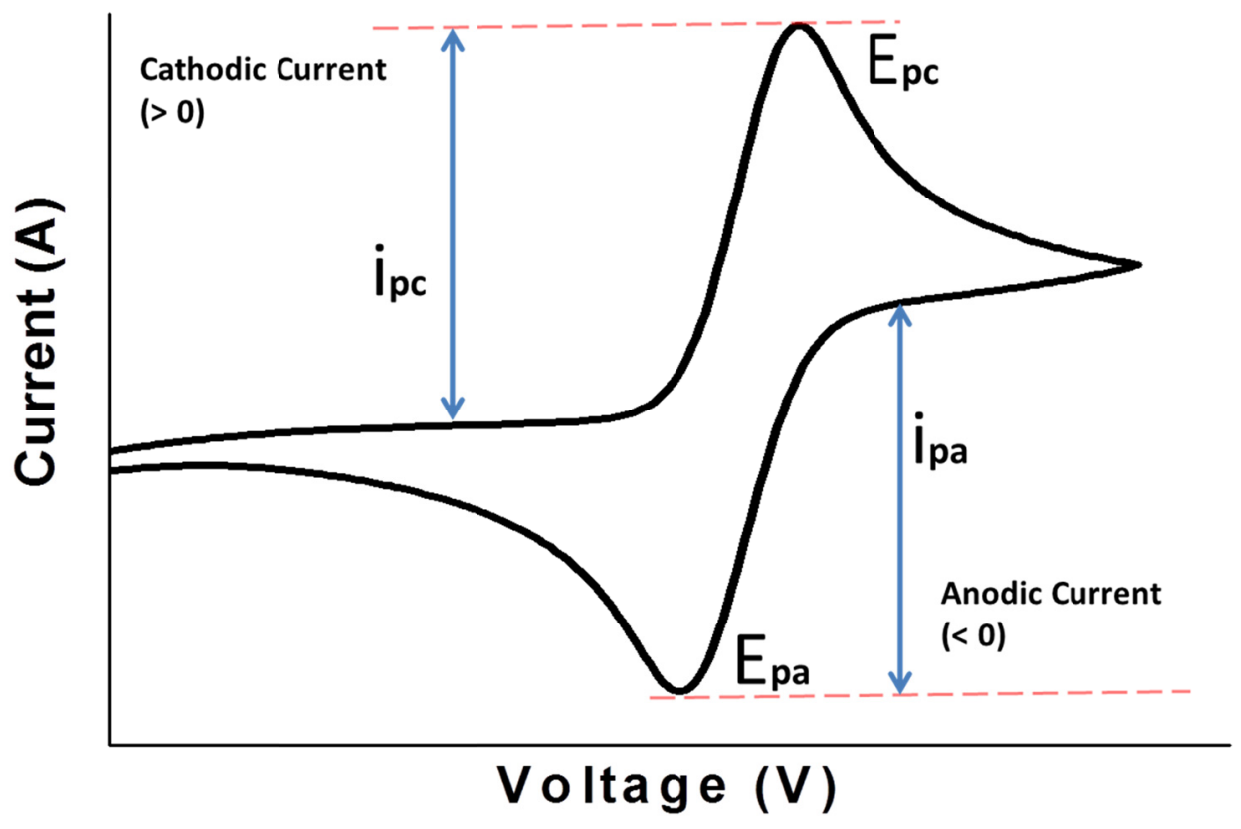

Fig. 2.8 A typical cyclic voltammogram with defined characteristics 
The Randles - Sevick equation[60] can be used to predict the concentration of the redox species from the peak current obtained in a $\mathrm{CV}$ for a reversible redox reaction.

$$
i_{p}=\left(2.69 \times 10^{5}\right) n^{3 / 2} A C D^{1 / 2} v^{1 / 2}
$$

Where $i_{p}$ is the peak current (in amperes), $n$ is the number of electrons passed per molecule of analyte oxidized or reduced, $A$ is the electrode area (in $\mathrm{cm} 2$ ), $D$ is the diffusion coefficient of analyte (in $\mathrm{cm}^{2} / \mathrm{sec}$ ), $v$ is the potential sweep rate (in volts/sec), and $C$ is the concentration of analyte in bulk solution (in moles $/ \mathrm{cm}^{3}$ ). Bhansali et al at the bio-MEMS and Microsystems research group at Florida International University have reported the development of electrochemical immunosensing of cortisol based on cyclic voltammetry[61-63]. A competitive electrochemical immunosensor for detection of cortisol and ACTH has also been reported[64].

Lastly, a review of Electrochemical Impedance Spectroscopy (EIS), an impedimetric electrochemical technique for immunosensing is presented. EIS is a highly sensitive technique for probing the features of surface modifications at the electrodeelectrolyte interface[65]. EIS can be used to detect the formation of an antigen-antibody immunocomplex through the highly sensitive change in the resistance (faradaic) and capacitance (non-faradaic) at the electrode surface[66]. The faradaic component arises from the electron transfer at the electrode-electrolyte interface by overcoming an activation barrier called the polarization resistance $\left(R_{p}\right)$ along with an intrinsic solution resistance $\left(R_{s}\right)$. The non-faradaic component arises from the formation of a double layer capacitor on the electrode surface. By sweeping the frequency of the applied voltage, 
frequency dependent behavior of the faradaic and non-faradaic components can be clearly distinguished. The measured impedance is the sum of the real part and imaginary part of the resistance, which can be represented in a Nyquist plot (real v/s imaginary) (Fig. 9a) or Bode plot (magnitude v/s frequency) (Fig. 9b). An electrical equivalent circuit, called the Randles circuit (Fig. 9a), can be used to extract the value of the parameter of interest. Several research groups are currently focusing their efforts on developing EIS based detection for immunosensing of E. Coli[67], cytokines [68], virus[69] and other clinically relevant biomarkers[70, 71]. Bhansali et al. are the first research group to detect cortisol in a label-free manner using electrochemical impedance spectroscopy $[7,72,73]$.

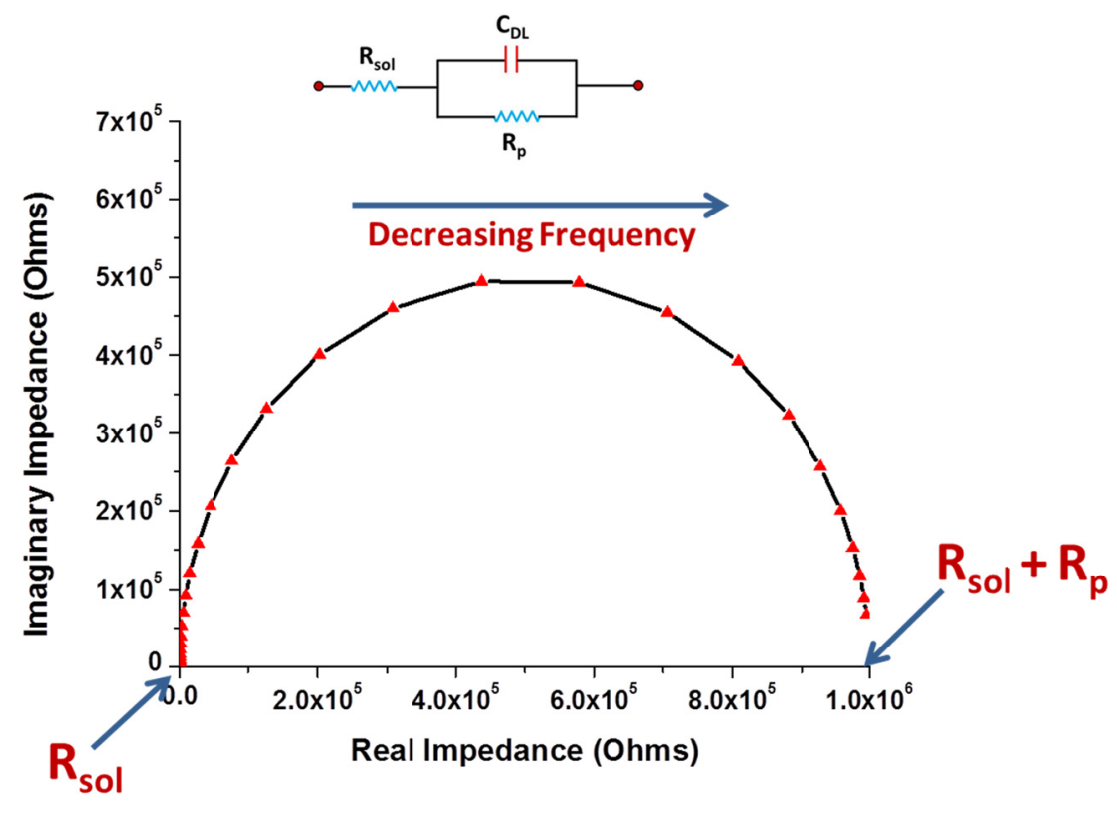




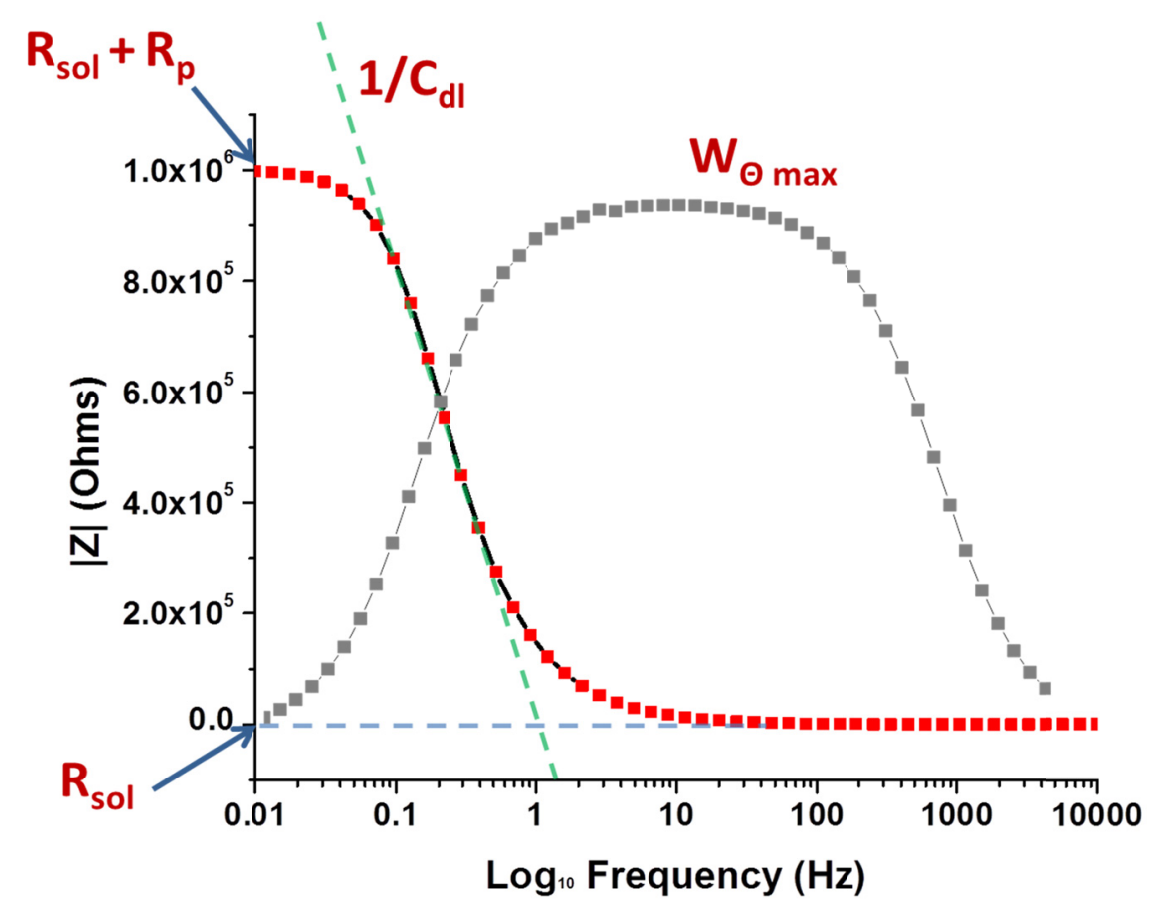

Fig 2.9 Electrochemical impedance spectroscopy data represented in terms of (a) Nyquist plot and (b) Bode plot.

\subsection{Nanomaterials}

In the last decade nanostructured materials have experienced a rapid development due to their potential applications in a wide variety of technologies such as electronics, catalysis, ceramics, magnetic data storage, structural components, optoelectronic devices, display devices, biomaterials and gas/bio sensors $[74,75]$. Such materials have shown peculiar and fascinating chemical, physical and molecular properties along with applications performance superior to those of their bulk counterparts [75]. For electrochemical detection, nanomaterial's play a very important role since they influence all aspects of electrochemical detection[76]. As mentioned earlier, immobilization of biological probes such as antibodies and DNA onto the sensor surface (conducting 
electrodes) requires highly specific binding chemistries, which may not be readily available on a native sensor surface. Nanomaterial's can be used to provide the required functional sites for binding the probes to the sensor surface. The use of nanomaterials also significantly increases the surface-to-volume function at the sensor surface thereby leading to a high efficiency of antibody immobilization. For applications requiring high sensitivity and low detection limits, signal amplification is a key requirement and nanomaterials play an important role in amplifying the electrochemical response through improved electron transport at the sensor interface. Depending on the application and sensing technique, the dielectric properties and electrical conduction of the nanomaterial's can be tuned to amplify the response of the electrochemical signal[77]. Nanomaterials commonly used for electrochemical immunosensors can be broadly categorized into Inorganic (Metal oxides, Metal nanoparticles, Graphene, CNT's etc), Organic (Conducting polymers, SAM's, Chitosan, etc.), and Composites (InorganicInorganic, Organic-Organic and Inorganic-Organic).

\section{Inorganic Nanomaterials}

Nanostructured metal oxides such as tungsten trioxide $\left(\mathrm{WO}_{3}\right)$, zinc oxide $(\mathrm{ZnO})$, tin oxide $\left(\mathrm{SnO}_{2}\right)$, titanium oxide $\left(\mathrm{TiO}_{2}\right)$, iron oxide $\left(\mathrm{Fe}_{2} \mathrm{O}_{3} / \mathrm{Fe}_{3} \mathrm{O}_{4}\right)$, silica $\left(\mathrm{SiO}_{2}\right)$ etc have been used as sensing materials[78-80]. At nanoscale, metal oxides possess properties that are significantly different from their coarse-grained polycrystalline counterparts, which leads to improved sensing characteristics[81]. A high degree of crystalline and atomically sharp terminations have made them very promising for a better understanding of sensing principles and for the development of a new generation of sensors. In these sensors, the 
surface effects dominate the bulk properties, because of enhanced properties such as catalytic activity and surface adsorption [79-82]. The utilization of these nano-platforms has reduced sensing instabilities caused by drift in electrical properties[83]. Metal oxide nano-crystals can be used as resistors, in field effect transistors (FET) and optical based sensors [79-82, 84]. These nanostructures have revealed good sensing properties and low power consumption in experiments, allowing for large scale manufacturing of wellorganized nanostructured sensor arrays $[85,86]$. However, better growth control is still required for widespread commercial applications [81, 82].

\section{Organic Nanomaterials}

Organic semiconductor nanostructures offer the possibility of creating tailor-made materials, for detection of a wide range of biomolecules. Organic semiconducting polymers have also been found to be suitable for microelectronic device fabrication, due to their excellent electrical characteristics [87-93]. These conducting polymers and other conjugated polymers have been used as the active layers in gas sensors since early 1980s [87]. The optical, electrical, and molecular properties of these continue to be improved, through synthesis of their composites and blends ensuring improved sensing applications [94-96]. Nanostructured conducting polymers such as polyaniline (PANI)[97, 98], polypyrrole (PPy)[99], polythiophene[100] etc. exhibit sensing behavior because of their desired functionality, conductivity and order. A detailed discussion on PANI is presented in chapter IV. Majority of the reports on these polymers is focused on tailoring optical and electrical properties at nanoscale [101]. Recent studies have highlighted the functional properties of conducting polymers for device fabrication. Many of the devices 
based on these nanostructured materials have shown poor sensitivity and slow response time because the functional properties were not fully understood [102]. Improved understanding of functional properties provides opportunities to synthesize new nanostructured conducting polymers that addresses the above [101, 103]. Another class of organic nanomaterials referred to as self-assembled monolayers (SAM) are discussed in detail in Chapter V.

\section{Organic-Inorganic Hybrid Nanocomposites}

Organic-inorganic hybrid nanocomposites are a fast growing area of research in advanced functional materials science. Significant efforts have been focused on the ability to obtain nanostructures of desired shape, size, and properties using innovative synthetic approaches for various applications[84]. The properties of hybrid nanocomposite materials depend not only on the properties of their individual constituents, but also on their morphology and interfacial characteristics [104-108]. The surface modification and functionalization of nanoparticles, covalent attachment, selfassembly, and ease of organization on the surfaces provides a means to generate nanocomposite materials with tunable surface properties [84, 104]. There is also a possibility of the realization of new properties that are unknown in the constituent materials. Efforts are continuing towards the preparation of new nanocomposite materials to obtain novel properties $[84,105,109,110]$. There is a strong interest in using nano metal/metal oxides as additives in the functionalization of polymers (conducting/bio polymer), and significant research activities have been undertaken in nanocomposite science for device applications[111, 112]. In nanocomposites, inorganic nanoparticles can 
be metal oxides, three-dimensional, two-dimensional, one-dimensional, and zerodimensional nanomaterial's. Experimental studies have shown that incorporation of mentioned inorganic nanomaterial's into the polymer matrices leads to organic-inorganic nanocomposites of enhanced and improved properties. Moreover, some new properties are also observed, that are absent in the counterpart molecules [113-115]. Additionally, they provide desired model systems for sensor fabrication. Therefore, nanocomposites have been used for a wide range of applications e.g., high energy radiation shielding materials, microwave absorbers, optical limiters, polarizers, sensors and hydrogen storage systems[114, 116-120] [84, 115, 116, 120].

Other than interfacial interaction, control of nanoparticle morphology is an important aspect that influences the nanocomposite performance. Hence in a nanocomposite development effort, a long-term goal of development of new synthesis strategies to control the size, shape and composition are very important. This control allows for the tuning of nanocomposite properties[112]. Depending upon the nature of the metal or metal oxide and polymer structure, these processes lead to the incorporation of inorganic nano-moieties into the polymer backbone via different electrostatic interactions[112]. Hybrid nanocomposite thin films containing metal or semiconductor nano-crystals and polymers exhibit porous structure[117]. This enables the biomolecules to readily adsorb onto the nano-crystals of the nanocomposite film and show a strong sensing behavior $[104,109,112,116]$. Recently, it has been demonstrated that it is possible to develop suitable techniques for these materials, which are often sufficient to yield high quality nanocomposite films $[112,118,120]$. 
In this research, focus has been on organic and organic-inorganic composite nanomaterials. Self-assembled monolayers (SAM) due to many attractive features such as desired functional group for immobilization of anti-bodies and binding to gold substrate, repeatable and stable fabrication process, good control over fabrication process and uniform morphology is chosen as the nanomaterial for functionalization of electrodes with anti-cortisol antibodies. Alternatively, an organic-inorganic nanocomposite of Polyanaline, a conducting polymer and $\mathrm{Ag} / \mathrm{AgO}$, metal/metal-oxide nanoparticles has been explored for demonstrating improved sensing range in a mediator free sensing scheme. The use of the hybrid nanocomposite is expected to increase the signal response due to the conducting nature of the nanomaterial and also improve the loading capacity of the anti-cortisol antibodies. The presence of redox sites on Polyanaline (PANI) results in the self-reduction and oxidation of PANI reducing the need for a redox probe (mediator) in the electrolyte. These topics are discussed in greater detail in further chapters.

\subsection{Microfluidics}

Microfluidics is an application of MEMS, dealing with the study and development of micro and nano-liter fluid volume based analytical devices[121]. Microfluidic systems offer many advantages over conventional bench top analytical systems such as small sample volumes, low energy consumption, utilization of micro and nano scale phenomenon, miniaturized form factors, portability, faster response time and disposability. These advantages of microfluidic devices make them the fundamental building block of point of care technologies. Microfluidics is a highly interdisciplinary field that brings together electronics[122], physics[123], biotechnology[124], optics[125], 
material science and chemistry[126].Typical microfluidic applications are focused on continuous flow devices for detection and counting of biological entities[127], biosensing[128] of protein biomarkers, microarray biochips for electrophoresis and polymerase chain reaction (PCR) amplification [129], optofluidic systems for tunable microlens arrays[130] and microfluidic fuel cells[131].

The biggest application that drives the development of microfluidics technology is point of care diagnosis[132, 133]. In this day and age, where early diagnosis can significantly bolster efforts to fight ailments ranging from lifestyle diseases (e.g. diabetes, stress), conditions arising from cancer, infectious diseases and bio-terrorism threats, the need for continuous monitoring of target analytes at point-of-care is imperative. Ideal point of care systems need to be portable, of low form factor, be disposable, biocompatible, produce instantaneous results from raw samples and yet maintain a low cost per unit[134]. Microfluidics offers the potential to satisfy all these demands. The point of care system finds applications mainly in the biomedical and environmental sensing domain. For biomedical applications, point of care systems are best suited for personalized health diagnostics for day to day monitoring of physiological variables, prognostic kits for cancer, and applications in third world countries, where complete medical diagnosis is unavailable.

Food safety and environmental pollution monitoring are becoming new priority areas for human health care in today's day and age. Materials such as carcinogenic organic solvents, toxic materials, and even nuclear contamination (e.g. recent earthquake in Japan leading to nuclear contamination in water bodies) which are very hazardous for 
environment and human health are found in packaged foods, water bodies and air. Also, a wide range of environmental problems such as global warming, ozone layer depletion, acid rain, increase in harmful waste materials in the environment, dioxin, and pollution of air and water require solutions. Therefore, environmental monitoring is crucial to protect public from toxic contaminants and pathogens that can be released into air, soil, and water. Point of care detection systems, which provide real-time quantification of environmental and health hazards, is the need of the hour[135].

\subsubsection{Microfluidic Flow}

Liquid flowing through a fully covered channel can be characterized using the Reynolds Number $\left(R_{e}\right)$, given by:

$$
R_{e}=\frac{L V_{a v g} \rho}{\mu}
$$

Where, $L$ is the critical length scale (typically the diameter of the channel), $V_{\text {avg }}$ is the average velocity of the flow, $\rho$ is the fluid density and $u$ is the viscosity of the liquid. Re is a non-dimensional number that is used to determine the flow regime of the liquid. A Re number below 2000 is considered laminar and above 2000 is considered turbulent in nature. Since the Re is directly dependent on the diameter of the microchannel, $\operatorname{Re}$ numbers are typically very small in microchannels, indicating laminar flow. In the laminar flow regime, the motion of liquid follows a predictable velocity pattern as opposed to turbulent flow indicating a highly random and chaotic flow pattern. Also, 
since surface to volume ratio increases by several fold at the microscale, surface forces such as surface tension, capillarity and Van der Waals forces dominate.

The Navier-Stokes equations, which are derived from the principles of conservation of mass (continuity), momentum (conservation) and energy ( $1^{\text {st }}$ law of thermodynamics), can be used to describe and predict flow of Newtonian fluids such as water and many biofluids through a control volume. With knowledge of the applied pressure differential $(\Delta \mathrm{P})$ and the average flow rate (Q), the Navier-Stokes equations can be solved for rectangular microchannel geometry in terms of fluidic resistance $\left(\mathrm{R}_{\mathrm{f}}\right)$, and is given by:

$$
\rho\left(\frac{\delta \mathbf{v}}{\delta \mathrm{t}}+\mathbf{v} * \nabla \mathrm{v}\right)=-\nabla \mathrm{p}+\nabla * \mathbf{T}+\mathbf{f}
$$

Fluidic resistance is an important parameter that can be numerically derived, as an indicator for resistance to flow of fluid in the microchannel. As can be seen from equation, the fluidic resistance is dependent on the geometry of the channel and plays an important role in the design of microfluidic channel geometries.

\subsubsection{Types of Microfluidic Flow}

\section{Capillary Flow}

At the microscale dimensions of the microchannel cross sections, capillary forces and surface tension forces play a significant role and can be utilized. The small cross section of a typical microchannel and the high surface-to-volume ratio creates a large enough capillary force to drive fluids in microchannels that are of typical lengths in 
microfluidic systems. An important contributing factor along with the geometry of the channel cross section is the surface energy of the channel walls that determines the hydrophobicity/hydrophilicity of the surface. A hydrophilic surface contributes significantly to the capillary force. Capillary drive flow can be a very inexpensive alternative to transport fluids, without the need for external bulky devices such as pumps. However, this method is severely limited by the microchannel design and does not allow for real-time control of flow rate and directionality.

\section{Pressure Driven Flow}

Pressure driven flow is generated by applying a positive pressure (infusion) or a negative pressure (withdrawal) to drive fluids in a microchannel. The flow profile of fluid in a microchannel induced by pressure driven flow behaves as Hagen-Poiseuille flow, displaying a parabolic shape function with maximum velocity at the center of the channel and zero velocity at the channel walls (Fig. 10a). This condition is referred to as no-slip boundary condition. Pumps that are used to create pressure driven flow are typically based on a stepper motor that is connected to a linear displacement transducer. The rotation steps in the stepper motor can be precisely calibrated as a function of flow rate.

\section{Electro-osmotic Flow}

Electro-osmotic flow relies on the movement of ions in a fluid closer to the channel surface. This is achieved by ensuring that the inner surface of the microchannel is appropriately charged either naturally or through surface modifications to add charge. Then, an electric field is applied along the length of the microchannel resulting in ions in 
the liquid moving towards the electrode of opposing polarity, in the process creating a bulk flow of the fluid. Unlike in pressure driven flow, the flow regime follows a plug-like profile, where a uniform velocity is maintained for every molecule in the fluid (fig. 10b). This method is preferred in applications such as capillary electrophoresis and electro chromatography for particle separation. A major drawback however is the need for charging the surfaces to enable electro-osmotic flow, which restricts microfluidic design to a selected few materials and applications.
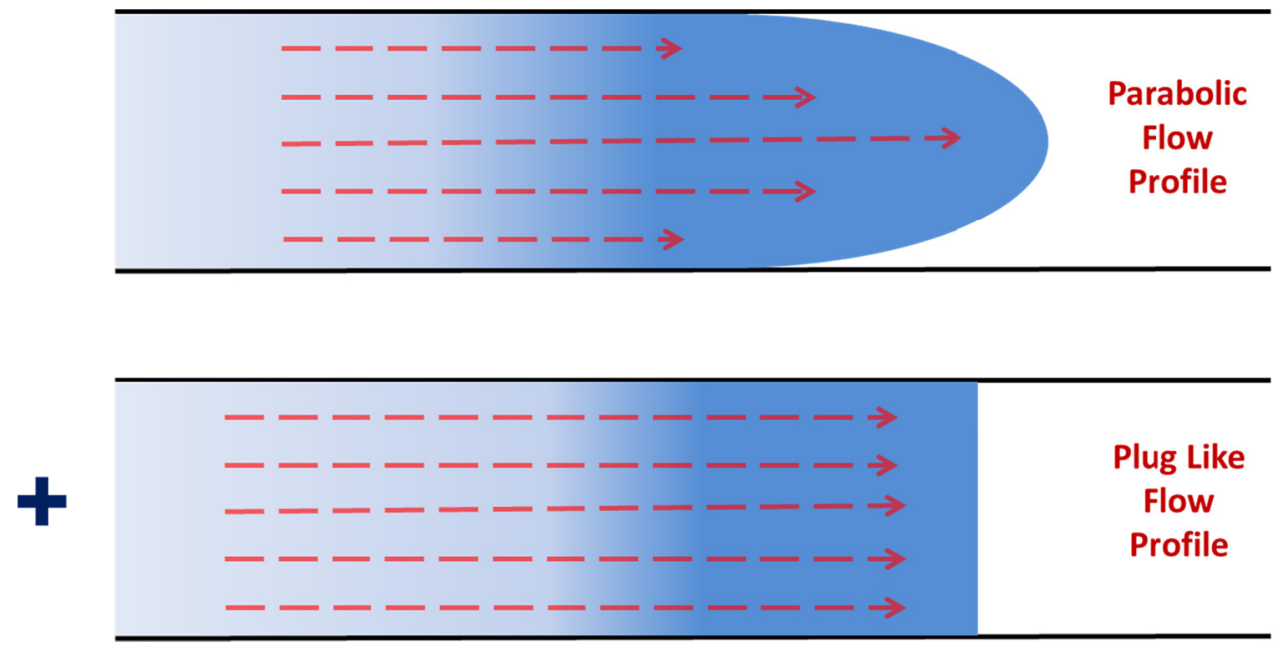

Fig 2.10 Schematic illustration of flow profiles seen in (a) Pressure driven flow and (b) Electro-osmotic flow.

Electrowetting Driven Flow

A more recent development in flow generation in microfluidics involves the generation and control of droplets and precise control on the directional movement of the droplets. This technique is generally referred to as droplet microfluidics and the enabling technology is called Electrowetitng-on-dielectrics (EWOD). Fig. 11 presents a schematic of the arrangement of electrowetting driven droplet movement. The set-up consists of a 
series of individually addressable electrodes buried under a very thin film of a hydrophobic insulator. With the droplet sitting on more than one electrode and the application of a voltage to only one of those electrodes, the contact angle of the droplet is reduced is artificially reduced only in one side of the droplet through an electrically induced change in the change in surface tension. This causes a pressure differential within the droplet leading to a bulk flow in the droplet to attain equilibrium position. By carefully activating-deactivating a series of electrodes, the droplet can be transported between two points. This technique has attracted attention due to advantages such as passive fluid transport and electrical control.

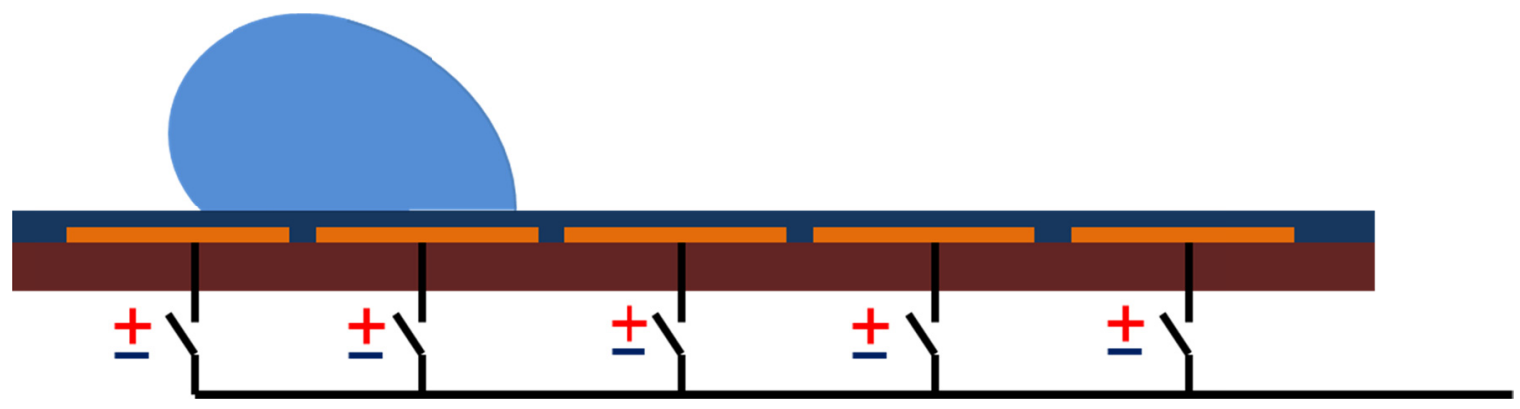

Fig 2.11 Schematic illustration of electrowetting of droplet for achieving droplet transport on individually addressable electrodes.

\subsubsection{Fabrication of Microfluidic Devices}

Silicon and Glass have long been the materials of choice for microfluidic device development. Silicon microfabrication using MEMS based processing techniques is very well characterized for prototyping microfluidic devices. MEMS processing involves a process of sequentially depositing, patterning and etching processes a silicon substrate. Fig. 12 presents a schematic describing the basic processes involved in MEMS based fabrication on silicon, glass and polymer substrates. 


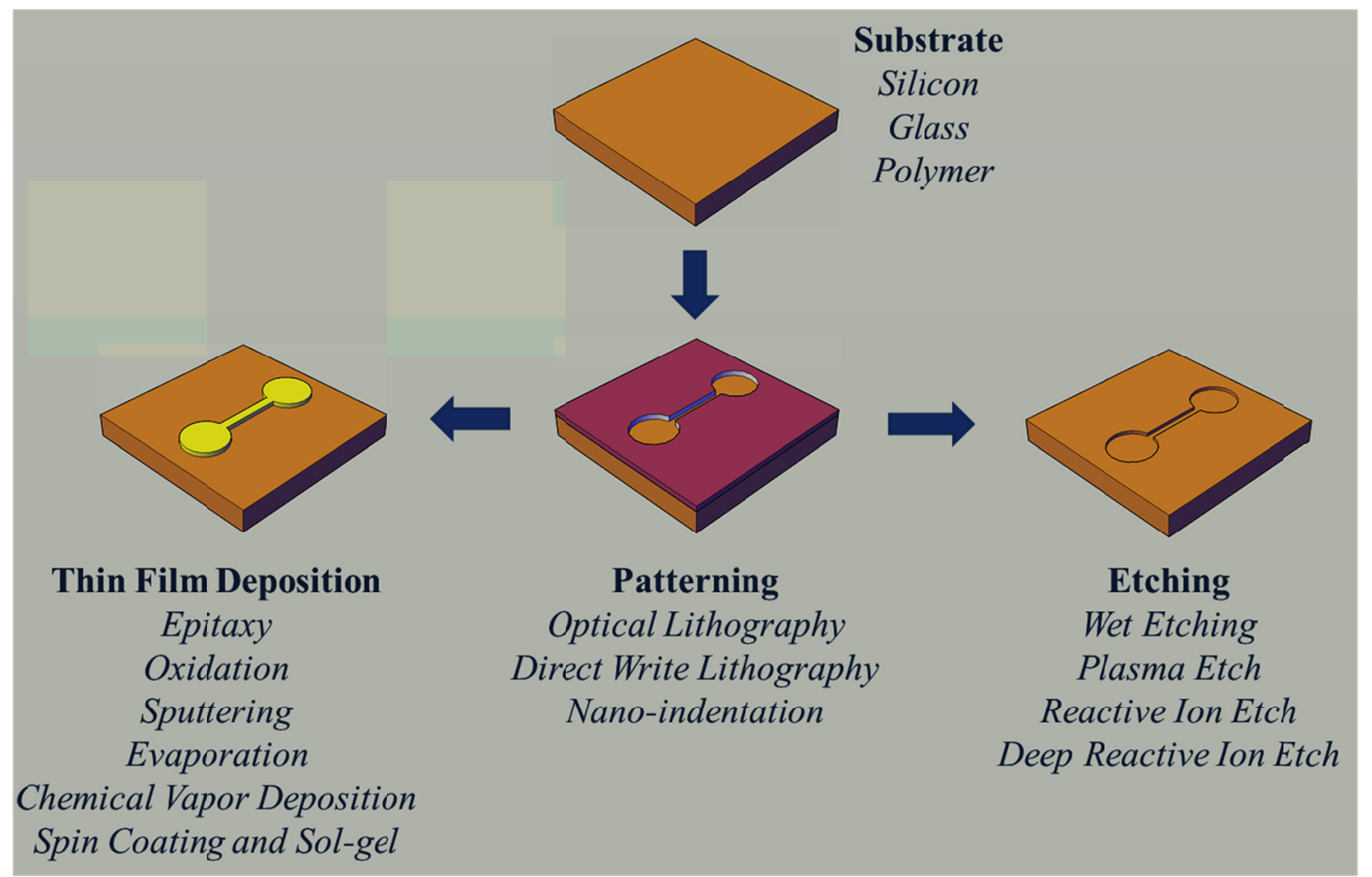

Fig 2.12 Schematic illustration of the three basic processing steps in microfabrication.

Silicon however has inherent drawbacks such as expensive cleanroom usage, opacity and impermeability to gases. Development in polymer science has led to improvement in processing of polymers leading to applications in several avenues. Whitesides et. Al [136] demonstrated the use of polydimethylsiloxane (PDMS), an organosilane, for the fabrication of microfluidic structures using soft lithography technique. Soft lithography involves a series of techniques that are based on replicating structures on the basis of master-mold patterning and stamping of thermally curable and photo-curable polymers. Fig. 13 presents the schematic explaining the simple technique of making micro-patterns in a curable polymer using a microfabricated master. 


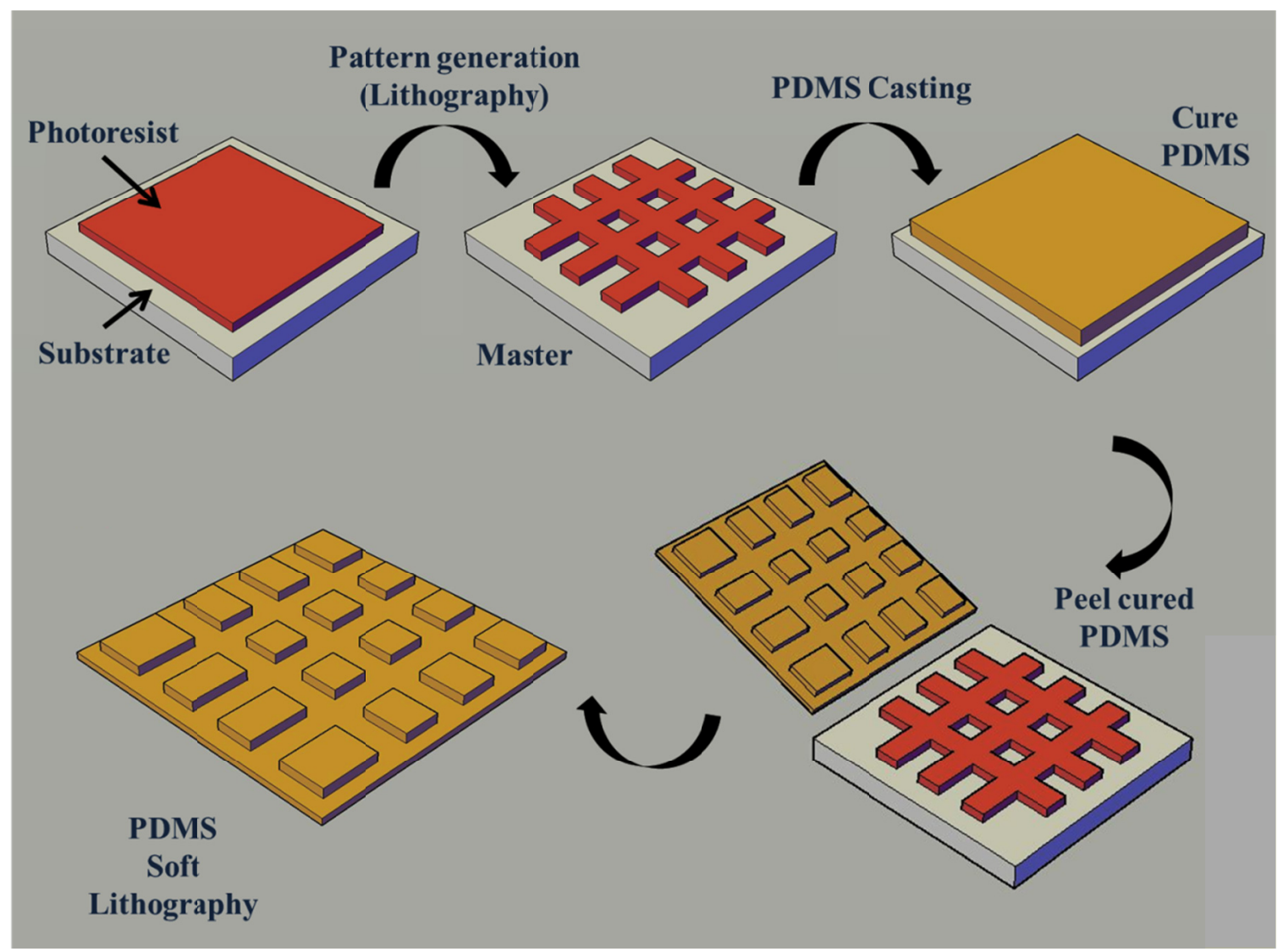

Fig 2.13 Schematic illustration of the processing steps in fabrication of PDMS structures using soft lithography.

Significant efforts went into the adaptation of polymer processing techniques such as injection molding, nano-imprint lithography and hot embossing for microfluidic device processing. Polymers such as PDMS, PMMA, SU-8, Teflon etc., have characteristics such as transparency, ease of surface modifications, low electrical and thermal conductivity, biocompatibility, and flexible substrates, that has made them attractive materials for demonstration of lab-on-chip applications[137]. Today, there is significant literature available on polymer materials based microfluidics; however, there remain many challenges in progress of the polymer based microfluidic chips into the next stage of technology evolution, which is commercialization. Silicon and polymer materials 
essentially use a lithography based fabrication technology which involves thin film deposition and etching techniques combined with photolithography to define patterns that create microfluidic structures such as channels, reservoirs, vias and valves. Lithography based approach confines the development of microfluidic structures to $2 \mathrm{D}$, requiring multiple lithography steps to create 3D structures. Alignment errors introduced during lithography and leakage in bonding of the multilayer microfluidic devices can cause device failure. Since the processing is sequential, failure during any one processing step leads to total process failure. While polymer materials are relatively inexpensive materials, only a handful of polymers are processable and cost of lithography-based fabrication is significantly high. Another perceivable drawback from polymer based microfluidic systems is the incompatibility for integration with on-chip support electronics.

Material selection is a crucial part of microfluidic system development as it impacts the processing, functionality, application and disposability of the sensor strips and the fluidic manifold. Processing related details such as fabrication technique, integration with support structures and hermiticity are directly affected by the material used. Functional aspects of a microfluidic device such as capillary forces in microchannels, hydrophobicity and nonspecific adsorption of the sample analyte are determined by the material chosen. The choice of material also determines corrosiveness, temperature isolation and hermiticity for applications in implantable sensors and harsh environments. 


\subsubsection{Low Temperature Co-fired Ceramics (LTCC)}

To overcome the challenges in current processing technology and materials available, newer materials are constantly being investigated to add novel functionality and processing capability for microfluidic device fabrication. Ceramic based microfluidics is one such approach that offers many novel structural and functional capabilities in microfluidic device design and fabrication. In recent time, low temperature co-fired ceramics (LTCC), a material used extensively in the IC industry as a packaging material over the last two decades, has gained attention as an alternative to glass, polymer and silicon for the fabrication of microfluidic systems[138-142]. LTCC offers a number of advantages over polymers, glass and silicon, making it an ideal material for developing microfluidic devices[143]. Since the beginning of 1980's, LTCC has been extensively developed as a microelectronic packing material in multichip modules. A representative LTCC device consists of a multilayer stack of sintered ceramic tapes, each of which contains passive electronic elements such as resistors, capacitors and inductors buried in it. The various layers are interconnected through via filled with conducting materials such as gold $(\mathrm{Au})$ and silver $(\mathrm{Ag})$ paste.

LTCC, due to its inherent properties such as chemical inertness, biocompatibility, high-temperature stability, low thermal conductivity, excellent dielectric properties, mechanical strength, packaging capabilities and three dimensional structuring lends itself well for the development of microfluidic devices[139, 144]. LTCC as a material for development of microfluidic based systems has shown promise in overcoming the important drawbacks of polymer microfluidic systems. LTCC based fabrication aids rapid prototyping with a significantly low turn-around time in a semi-clean room 
environment with minimal use of expensive tools compared to conventional clean room based microfabrication techniques[145]. The parallel processing of the multiple layers and final integration into a multilayer stack easily accommodates design modification during initial device development. The maturation of the microelectronics packaging technology using LTCC allows for integration of the fluidic system with the support electronics needed for microfluidic operation and automation[138]. The sintering temperature of LTCC $\left(850^{\circ} \mathrm{C}\right)$ allows for integration of sensing and actuating electrodes using precious metals such as $\mathrm{Au}, \mathrm{Ag}, \mathrm{Pd}$ and $\mathrm{Cu}$. This is a significant advantage over high temperature co-fired ceramics (HTCC) which allows only the use of refractory metals such as $\mathrm{W}$ and $\mathrm{Mo[146]}$. At reasonable feature sizes, the integration of the working metals such as $\mathrm{Au}, \mathrm{Ag}$ and $\mathrm{Cu}$ can be performed using a standard screen printing process. The relatively simple and inexpensive fabrication methods also lend itself to economical manufacturing in large scale. The multilayer approach accommodates the incorporation of three dimensional structures and high aspect ratio features such as overlapping microchannels, which is otherwise a tedious and complicated process in lithography based microfabrication requiring repeating steps of sequential lithography. Leakage, one of the common challenges faced in microchannel bonding to substrates is a non-issue in LTCC microfluidics since all the layers are co-fired, resulting in a leak free, compact microfluidic manifold. LTCC, post fired, is also a mechanically stable, swell free, corrosion resistant material.

In terms of the material properties, LTCC demonstrates an equal or better performance compared to silicon, polymers and glass. Table.1 provides a comparative summary of the relevant properties of LTCC, Silicon, Polymer and Glass. 


\begin{tabular}{|c|c|c|c|c|}
\hline & $\begin{array}{c}\text { LTCC } \\
\text { DuPont 951 }\end{array}$ & $\begin{array}{c}\text { Silicon } \\
<\mathbf{1 0 0}>\end{array}$ & PDMS & $\begin{array}{c}\text { Glass } \\
\text { (Borosilicate) }\end{array}$ \\
\hline Mechanical & 320 & 700 & 2.24 & 69 \\
\hline Flexural Strength (MPa) & 120 & 140 & 0.0018 & 64 \\
\hline Young's Modulus (GPa) & 3.1 & 2.329 & 0.97 & 2.23 \\
\hline Density (g/cm $\left.{ }^{3}\right)$ & $13,13,15$ & - & - & - \\
\hline Shrinkage (X, Y, Z) \% & 5.8 & 2.7 & 310 & 3.2 \\
\hline Thermal & 3 & 120 & 0.15 & 1.1 \\
\hline Expansion Coefficient (10-6/ C) & $7.8(10 \mathrm{MHz})$ & 2.32 & $2.3-2.8$ & $4.6(1 \mathrm{MHz})$ \\
\hline Conductivity (W/mK) @ (25-300C) & $>10^{12}(100 \mathrm{~V} \mathrm{DC})$ & $>10^{5}$ & $>10^{14}$ & $>10^{15}$ \\
\hline Electrical &
\end{tabular}

Table 2.1 Comparative table of the properties of LTCC with silicon, PDMS and Glass.

Low temperature co-fired ceramic (LTCC) and high temperature co-fired ceramic (HTCC) are the two raw ceramic substrates used in the manufacture of multilayer ceramic substrates. The primary difference between the two comes from the material composition and firing temperature of the two materials. The main constituent of HTCC ceramics is $\mathrm{Al}_{2} \mathrm{O}_{3}$, which requires a high sintering temperature around $1500 \mathrm{C}$. The high sintering temperature used for HTCC processing poses limitations, which include incapability to integrate passive electronic components such as resistors and capacitors and use of refractory metals only. LTCC, adapted from the HTCC technology, is an amalgamation of Al2O3 (45\%) and glass (40\%) buried in an organic binder (15\%) [146].

The constituent materials and their proportions are chosen to thermally match the sintering temperature of $850 \mathrm{C}$, which is significantly lower than the HTCC sintering temperature. The lowered sintering temperature allows for the integration of resistors and capacitors and also the co-firing of screen-printed metal on the ceramic substrates. A sintering model for the ceramic-glass composite system has been proposed by GongoraRubio et. al.[139]. The unfired ceramic tapes consist of A12O3 and glass granules 
dispersed in an organic binder. At sintering temperature, the glass granules melt and surface tension and capillary forces facilitates the encapsulation of the sintered $\mathrm{A} 12 \mathrm{O} 3$ granules. Annealing results in the formation of a dense ceramic structure.

\subsubsection{LTCC Processing}

The unfired ceramic material is available in the form of sheets and is referred to as green tape, the color green representing the unfired state of the ceramic. Fig. 14a presents a schematic of a standard LTCC fabrication process. The process starts by defening the substrate size and number of layers. In LTCC fabrication, all the layers that constitute the final device are processed parallely. After the substrate size is determined, the design features such as channels, grooves and vias are created using patterning tools such as laser cutting, punching heads, jet-vapor ethcing and CNC machines. Feature size is dependant on the patterning tool used with typical feature sizes in the 10 to $100 \mu \mathrm{m}$ range. Conducting vias used as interconnects across layers are formed by filling the punched vias with conducting inks of $\mathrm{Au}$ and $\mathrm{Ag}$. Electrical conduction lines and sensing/actuation electrodes are formed by screen printing. The individual layers are then stacked using an aligning chuck and a prefiring lamination step is performed at a pressure of $3000 \mathrm{psi}$ and temperature of $150 \mathrm{C}$ for $8-10$ minutes. The lamination step is performed to temporarli bind all the layers while in the aligning chuck. The laminated LTCC device is then sintered in a programmable furnace. The temperature ramping profile is a critical parameter during sintering with slow ramping rates recommended to avoid thermal stresses and shrinkage mismatch between layers. At lower temperatures (200 to 400C), the organic materials burn out, and this step is critical for successful fabrication of 
microfluidic structures such as microchannels and vias and is discussed elaborately in the next section. At the sintering temperature of $850 \mathrm{C}$, the glass granules melt and encapsulate the ceramic granules forming a homgenous structure across all the individual layers. Fig. 14b presents a typical firing profile for Dupont 651 and Ferro A6 green tape.
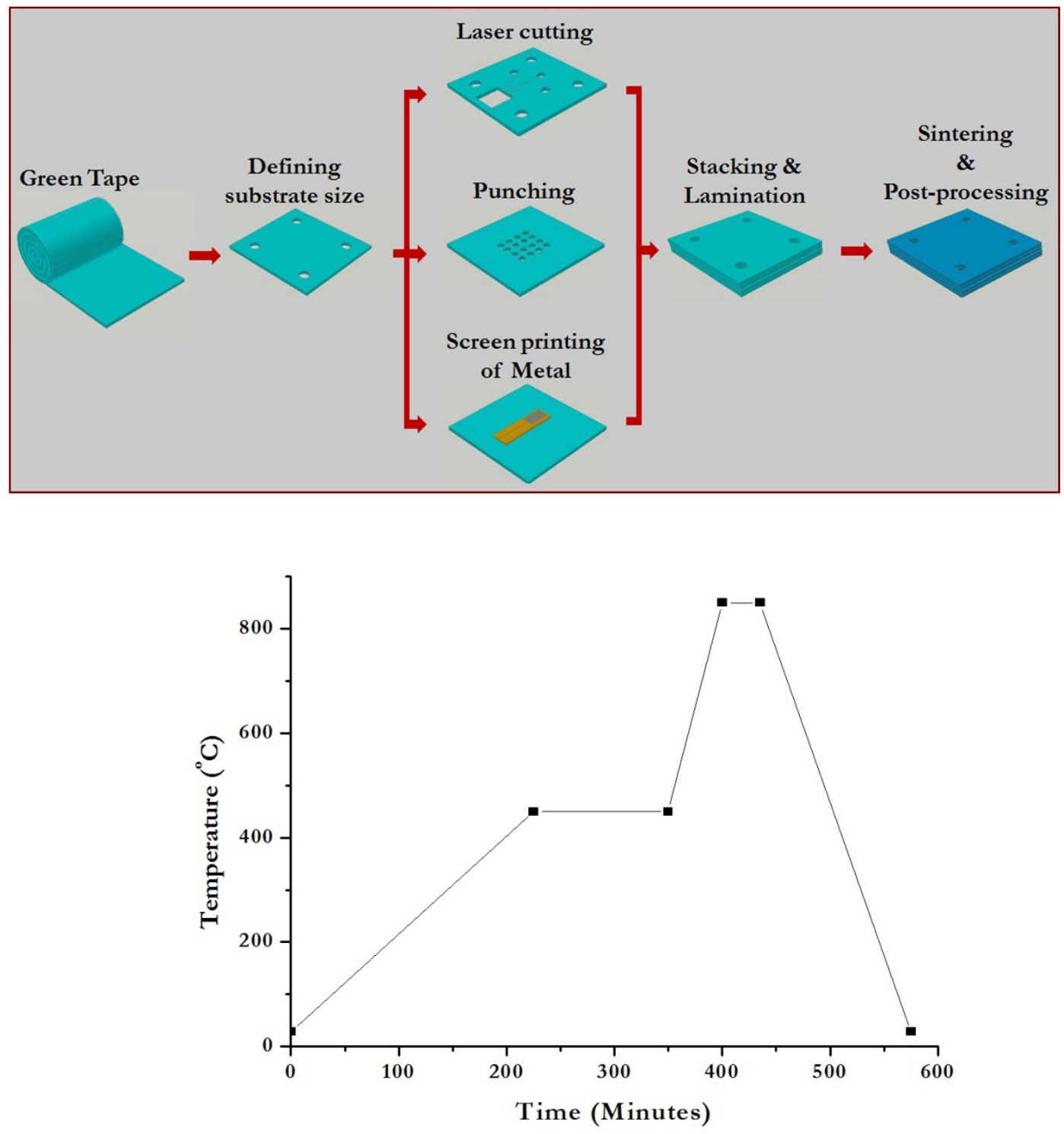

Fig 2.14 (a) Schematic illustration of the processing steps in LTCC based microfabrication; (b) Temperature ramp profile during the sintering process of LTCC structures. 
The burning out of the organic materials, which constitutes about $15 \%$ of the total composition of the green tape results in a proportional shrinkage in the overall dimensions of the paternened structure in all the three dimensions. Hence, the shrinkage factor is always deliberated into during the initial design of all the critical features. Shrinkage free LTCC tapes are also available from commercial vendors such as Heralock and ZST (Sea Ceramic Technologies).

\subsubsection{Fabrication of Microfluidic Components in LTCC}

\section{Microchannels}

The fabrication of microchannels is one of the core processes in the development of microfluidic devices. Ideal design features of microchannels include vertical side walls, zero dead volumes, leak free connection to inlet and outlet fluidic connectors, leak free bonding to adjacent layers in multilayer architectures, low surface roughness and uniform cross section across the length of the channel to maintain the dynamics of the fluid flow.[147] The most compelling problem faced during microchannel fabrication using LTCC technology is the occlusion of the microchannels due to collapsing of the LTCC layers during lamination. To overcome this challenge, many modifications to the standard LTCC process flow have been reported. One of the popular approaches to overcome the channel sagging, or collapsing, has been to use a sacrificial material to fill the empty spaces in the microchannels prior to lamination[148]. During the lamination process the sacrificial material provides the mechanical support required to maintain the structural integrity of the embedded microchannels and during the sintering process, the sacrificial materials burn out leaving a void that forms the empty microchannel. 
Golonoka et. al[149] have reported the characterization of an LTCC based microchannel fabrication process using carbon-black paste and cetyl alcohol as the sacrificial materials with the DuPont DP 951 green tape. A similar characterization of the microchannel fabrication process has been reported using high purity carbon paste, carbon tape and cetyl alcohol with zero-shrinkage green tapes from Heraeus HL2000[150]. Scanning electron microscopy (SEM) has been used to characterize the cross section of the microchannels fabricated using the different sacrificial materials as fillers. Significant improvements have been demonstrated leading to decreased sagging and channel constriction. The use of sacrificial materials requires a well-characterized temperature ramping profile during sintering to allow the complete burnout of the sacrificial material to avoid residual retention in the microchannels after sintering. An alternate technique for overcoming the channel collapse and sagging issue, without the use of any sacrificial materials has been reported by Shafique et. al [151]. The technique uses the method of progressive lamination, where in, the lamination of the layers is performed multiple times individually at low pressures and also after aligning the layers in a progressive manner. This method, implemented successfully, provides a few notable advantages. Since the pre-lamination and post-lamination process is identical, the overall process flow is not disturbed. The process is also considerably simplified by overcoming the use of sacrificial materials. The progressive method of lamination may however lead to a poor binding between the pre-laminated individual layers and can cause delamination of the layers during sintering. The delamination can lead to leaky channels and can cause bonding and peeling issues at integration and packaging stages. To overcome issue arising from channel collapse and delamination, more recently, Deisinger et al [152] have 
reported the usage of low temperature, low pressure lamination along with double sided adhesive tapes as the sandwich between the ceramic layers. This modification allows for the fabrication of cavities with very low aspect ratio $(0.5 \mathrm{~mm}: 15 \mathrm{~mm})$ with no observable sagging. $\mu \mathrm{CT}$ (Computer Tomography) has been utilized here for the non-destructive analysis of the internal features of the multilayer ceramic structure. Maeder et al. [153] have reported the characterization of various compositions of hot melt adhesives as an alternative to adhesive tapes. The potential advantage of these hot melt adhesives is that the adhesives can be applied as thin film on the ceramic substrates in its green state and the subsequent processing steps for LTCC microfluidic device fabrication are not affected.

\section{Optical Windows}

A perceivable drawback of using LTCC based microfluidics is opacity of ceramic material. Transparency is a critical factor for applications involving optical detection such as fluorescence in $\mu \mathrm{TAS}$ systems. One of the approaches to overcome this challenge has been the use of a hybrid LTCC-Polymer or LTCC-Glass systems, in which, the microchannel and the underlying structure is made from LTCC and the top most layer that covers the microchannel and consists of the $\mathrm{i} / \mathrm{o}$ fluidic ports, is made from a transparent substrate such as PDMS or Glass. A technique for the bonding of LTCC substrates to PDMS has been reported by Malecha et. al. [154]. The laminated LTCC structure is first coated with a glaze layer using screen printing[155]. The glaze layer essentially forms a glass coating after the sintering process. Oxygen plasma exposure, that creates free radicals on the surface of PDMS and Glass that assists in the formation 
of covalent bonds between the two materials, is a well characterized process to obtain irreversible bonding for PDMS-PDMS, PDMS-Silicon and PDMS-Glass [156]. The same approach has been used to bond the PDMS cover to the glass covered LTCC substrate. The bonding between the glass covered LTCC substrate and PDMS provides a mechanically durable, leakage free, irreversible bonding. Pawel et. al. [157] report the integration of transparent glass windows into the LTCC substrate to aid in optical applications of LTCC microfluidic devices. A thin glass wafer is used as one of the layers in the LTCC stack. During the sintering process, temperatures in the $670{ }^{\circ} \mathrm{C}$ to $720{ }^{\circ} \mathrm{C}$ range initiates the softening of glass, but the surface tension forces of the molten glass help in maintaining the structure of the glass sheet. At the LTCC sintering temperature, glass is conformal enough to accommodate the shrinkage of the LTCC substrate, while being strong enough to maintain its shape. This advancement lends itself well to the development of LTCC devices for optical sensing and actuation applications.

\section{Simulation of fluid behavior}

Fluid flow behavior in microchannels has been a key point of focus in the area of microfluidics research [123]. Microchannel geometry, surface roughness, hydrophobicity, surface tension and viscosity of the fluid are some of the factors that determine fluid dynamics in microchannels. Rapid and complete mixing of buffers, analytes and reagents is a critical requirement for chemical analysis and biosensors based microfluidic systems. By understanding the fluid dynamics in microchannels and with careful design of the microchannel geometry, passive mixing of fluids can be achieved. The opacity of LTCC material can cause issues while characterizing fluid flow in microchannels. Especially in 
micromixers and microreactors, where determining the mixing efficiency and the effect of microchannel geometry on mixing is important, flow characterization is not straight forward. Computational fluid dynamic (CFD) simulation can be a very useful tool in predicting fluid behavior and aid in design of efficient microchannel geometry. Malecha et. al. [158] have reported the fabrication of microfluidic micromixer using LTCC technology. A detailed computational fluid dynamic (CFD) modeling of fluid flow under (a) steady-state, laminar flow and mixing of incompressible fluids and (b) an application example of two chemicals, 1- and 2- naphthols has been reported. The findings from the CFD modeling, such as the effect of inertial and viscous forces on mixing efficiency, have been experimentally verified using a prototype I-shaped serpentine microchannel fabricated using standard LTCC processing. The aid of modeling and simulation has also been used to study the deformation and failure of LTCC layers to design and obtain optimal microchannel dimensions. The use of computer modeling to understand fluid flow behavior in LTCC microfluidic devices has also been presented by Schlottig et. al. [159]. The network approach based model which describes stationary flow in LTCC channels of non-circular cross sections, is essential due to the variance introduced in microchannel cross section and geometry fabrication using LTCC processing. Computational modeling has also been utilized to optimize application specific microfluidic designs. In an effort to optimize the microfluidic design for an optic fiber based optofluidic system, Malecha et. al. [160] have reported CFD simulations to understand the effect of fluid inlet and outlet spacing on the transmission of light in the microchannel. The set-up consists of three different microchannel designs ( $\Pi$ type, $\mathrm{L}$ type and I type) with varying inlet and outlet configurations. The CFD simulation of fluid flow 
in the three different configurations indicate that the L type and I type structures showed decreased signal due to localized disturbances in fluid flow causing significant repeatability issues. An experimental approach for characterization of fluid flow in LTCC microchannels has been reported by Groß et al.[161]. Pressure drop method, which uses the pressure drop along the length of the microchannel and its corresponding friction factor to calculate flow rate as well as mixing efficiency. Another method described is the resistance time distribution measurement, which is based on the residence time of a liquid packet in a microchannel and its effect on mixing efficiency and yield. A pulse trace assembly is used to inject pulses of the experimental liquid and post processing on the recorded pulses and signal is used to understand the fluid flow behavior and mixing efficiency.

\subsubsection{Applications of LTCC Microfluidic Systems}

\section{Microreactors}

Microfluidic microreactors are increasingly gaining importance in biosensors, chemical synthesis and pharmaceutical research applications due to the many advantages such as excellent control over process parameters (temperature, $\mathrm{pH}$, and humidity, etc.). Microreactors also aid in pilot small volume R\&D activities, which is typical in chemical synthesis and drug discovery applications[162]. With the need for point of care systems gaining ground, microreactor technology has found good application in biosensing and PCR based systems, which require highly controlled environments in a microchamber [163]. Microreactors require the integration of multiple sensing and actuation components such as micromixers, microextractors, microheaters, microsensors for 
temperature, $\mathrm{pH}$, humidity and redox measurements and many more depending on the demands of the application[164]. Polymerase chain reaction (PCR) is a powerful biotechnology tool finding many applications in the fields of science and medicine. PCR can be used to identify genetic diseases, detect infectious diseases, identify cancer types and also in forensic sciences for high accuracy matching and identification of human samples. PCR essentially creates multiple copies of a target DNA fragment through a sequential thermal cycling process. Traditionally, the PCR process is carried out in a laboratory using vials, however, advancement in microfluidics and microsystem fabrication technology has allowed the creation of portable and compact devices that can be used at point-of-care and can produce real time results known as real-time PCR (rtPCR). The success of microfluidic PCR systems is highly dependent on the creation of three temperature zones on a single chip with high stability, while being thermally isolated from each other. Typically, metals such as nichrome and scandium are microfabricated onto the substrate to form microheaters, and the samples are transported between the thermal zones using microchannels. Silicon, glass and polymers have been previously used as materials to create microfluidic channels in PCR systems. LTCC, due to its high thermal isolation, biocompatibility and microfluidic processing capabilities can prove to be a better candidate for PCR applications. Malodobra et al. [165] have reported a study comparing the specificity, sensitivity and efficiency between traditional PCR systems and LTCC based PCR systems. Bembnowicz et. al. [166]have reported the development of a LTCC based microfluidic system for DNA amplification and measurement using PCR. The LTCC system consists of a microchamber which forms the integral part of the microreactor. Temperature control is provided using a thermoelectric 
module and a micro-thermistor for measurement. The detection system, optical based, consists of a fiber optic laser light source that is integrated into the LTCC chip and a CCD camera captures the fluorescent light emission using special filters. The developed system has been successfully tested for DNA measurement. An improvement to the above mentioned LTCC PCR system has been reported[167]. Here, the temperature control which consists of the microheater and the sensor are integrated into the LTCC substrate, thereby creating a more compact form factor for the envisioned system. A commercially available SMD (surface mount device) resistor is used as the microheater and a Pt100 element is used as the temperature sensor. Along with the heater and sensor, the support electronics are soldered onto the LTCC substrate. An additional metallic layer is screen printed to improve the thermal conductivity and achieve uniform temperature distribution in the microchamber. In the above two reported developments, the sample is held stationary in the microchamber, while the temperature is cycled between three distinct temperatures using the embedded heaters. Another popular configuration for PCR microfluidic systems is to sequentially transport the sample between three chambers, each maintained at the required temperature. Sadler et. al. [168]have reported the development of an LTCC platform for PCR and DNA detection using a continuous flow microfluidic system. Microchannels connect the three temperature zones which are realized using screen-printed Ag-Pd. Temperature sensors are surface mounted onto the LTCC substrate. One of the salient features of this design is the introduction of air-gaps between the parallel microchannels to provide thermal isolation during sample transport between the temperature zones. The functionality of the LTCC based continuous flow microfluidic system is demonstrated by performing real-time PCR and also DNA detection. Another 
fine example of the 3D architecture capability of LTCC processing has been demonstrated in an LTCC based microfluidic microreactor developed by Smetana et al $[169,170]$. The system consists of a spherical reactor cell for accumulation and mixing of reagents and other solutions. The system also consists of sensor arrays for monitoring of pH-, oxygen-, temperature and iodide sensitive sensors. A surface mount optical glass fiber is used reaction monitoring using absorption or fluorescence spectroscopy analyses. Temperature control is provided by pumping a thermal fluid through embedded ducts. A network of micro-channels with cross-section dimensions varying from $200 \mathrm{~mm} \times 200 \mathrm{~mm}$ up to $2 \mathrm{~mm} \times 2 \mathrm{~mm}$ is connecting the different measuring sections within the ceramic module.

\section{Whole Cell Sensors}

Cell cultures have been a great source of information for understanding the growth dynamics in the human body. Cell cultures are extremely sensitive to environmental variables such as temperature, $\mathrm{pH}$, humidity, ion concentrations, nutrients and pathogens in the culture media. Monitoring, regulation and response of cell cultures to triggers is a huge area of research that acts as enabling feature in drug discovery and toxicology. An LTCC based microelectrode array for monitoring of cell cultures has been reported by Ciosek et. al[171]. The microelectrode array is realized by screen printing of Pd-Ag paste and electrochemical deposition of $\mathrm{AgCl}$. The microelectrode array is functionalized with ion selective membranes of high selectivity for monitoring and detection of various cell culture variables such as cell culture media change, cell growth rate and toxicity levels. Electromagnetic force (EMF) is utilized to detect the changes in 
electrode response due to the ion concentration variation. Principle component analysis (PCA) is used to segregate the multiplexed data obtained from the sensor array. Bioparticle detection and counting has come into focus in the modern day due to the high potential of impact on daily life due to presence of harmful chemicals and biological elements in the environment and other consumables. Bio-terror threats and spreading of allergens and bacteria in public places such as airports also require deployment of sensors that monitor and detect bioparticles. Flow cytometry is one of the most successful technologies used to detect particle concentration and size in a rapid manner. Malecha et. al.[172] have reported the development of a LTCC based micro cell analyzer for detection and counting of bioparticles. The LTCC based system works on fluorescenceactivated detection in a flow cytometry set-up. The ceramic microfluidic system consists of a microfluidic chamber, microchannel, optic fibers, light emitting diode (LED) and photo detector embedded into the LTCC substrate. The integration of all the active and passive components of the system into a single substrate provides a form factor that is realistic for commercial applications. E. Coli and Saccharomyces cervisiae cells are used to demonstrate the functioning of the developed micro cell analyzer.

\section{Biosensors}

Biosensors are integrated small devices that employ a biological element such as an antibody, enzyme, receptor protein, nucleic acid, and cell or tissue section as the sensing element. Typically, a nanostructured matrix such as self-assembled monolayer, carbon nanotube, metal oxide or other nanocomposite material is used to immobilize the sensing element onto the detection surface. Various transduction techniques such as 
electrochemical, optical, acoustic, piezoelectric and gravimetry can be employed to quantify the concentration of the analyte that is sensed. Biosensors are a promising technology due to inherent advantages such as high selectivity, linearity, simple set-up, electrical output, high sensitivity and easy system integration. The sensing of various biomarkers using biosensors has been extensively researched. In recent years though, the focus has shifted towards integrating the developed sensors into a complete system that supports the sequencing of the sensing assays with the supporting electronics for data acquisition and analysis. LTCC technology, which facilitates the integration of microfluidic structures and electronic components such as resistors onto a single substrate, and the addition of integrated circuits by surface mounting provide the most suitable platform for developing miniaturized biosensing systems for a plethora of sensing applications. Immunosensors, which use antibodies as the bioreceptor, are highly preferred due to the high degree of selectivity and sensitivity that is associated with antibody-antigen interactions. Antibody immobilization strategies are very well characterized and are highly suitable for various transduction mechanisms such as electrochemical and optical due to superior electron transfer and secondary fluorescence antibody tagging properties respectively. For the fabrication of immunobiosensors, the availability of a surface that is conducive to antibody binding is a pre requisite. Typically, the antibody is immobilized at target areas such sensor electrode surface, while attempting to keep the peripheral areas free of any non-specific binding. Nanomaterial based immobilization matrices are used to improve surface to volume ratios, enhance the sensing response and also where the direct binding between the antibodies and the sensor surface is not feasible. With LTCC being investigated as a potential substrate material for 
the development of biosensing platforms, determination of optimum surface conditions is imperative. In this regards, Fakunle et al. [173] have evaluated the feasibility of using LTCC as a substrate for immobilization of antibodies on screen printed gold for electrochemical detection. Parameters such as pretreatment conditions (piranha cleaning $\mathrm{v} / \mathrm{s}$ water), electrochemical response of the screen printed gold electrodes and deposition of a nanomaterial immobilization of matrix such as self-assembled monolayer (SAM) on the electrodes were characterized and optimized. Electrochemical detection of Mouse IgG antigen using a sandwich type immunoassay was used as a model to demonstrate the feasibility of using LTCC as a suitable substrate material for the development of immunosensors.

Fakunle et al. [174] have recently reported the development of a complete microfluidic system that adopts the screen printed gold electrodes (previously discussed) into a multilayer LTCC microfluidic manifold containing microchannels. The sensing electrodes are incorporated onto all the four walls of microchanne. Enzymatic biosensors are also a popular choice of bio-receptors due to their rapid, efficient and selective detection of important analytes such as glucose, cholesterol, urea and lactic acid. Enzymatic biosensors have found success in commercialization and have proven their potential for point of care diagnostics and personalize health care. Malecha et al.[175] have reported the use of an enzyme based LTCC microfluidic biosensing platform for the detection of urea in biological fluids. The system design consists of a micro reactor chamber, an integrated micro heater and temperature sensor. Micro glass beads are used as the enzymatic carrier to determine the concentration of urea by measuring the $\mathrm{pH}$ at elevated temperatures. The enzymatic reactions are enhanced at elevated temperatures 
(37C) using the screen printed Pt micro-heater electrodes. The temperature sensors are realized by screen printing of a resistance-temperature dependent ink (DP 3630). The developed sensor is successfully tested for urea concentration determination in biological fluids. The system also demonstrates impressive shelf-life and repeatability (18 days). Another example of a LTCC based enzymatic biosensor has been reported for the continuous monitoring of glucose[176]. The detection system is based on the oxidation of glucose in the presence of the enzyme glucose oxidase, Gox. Hydrogen peroxide, a byproduct of the oxidation reaction is used to quantify the concentration of glucose. The sensing system, based on electrochemical (amperometric) measurements, consists of thick film working, counter and reference electrode, a mircoreaction chamber and a semi permeable dialysis tube running along the length of the device. The system operates by the size selective diffusion of the glucose molecule through the semi permeable walls of the dialysis tube into the micro reaction chamber. Glucose concentration up to $9 \mathrm{mM}$ was successfully quantified at a high sensitivity of $147 \mathrm{nA} / \mathrm{mM}$.

\section{Environmental Monitoring Sensors}

Owing to rapid globalization, a wide range of man-made chemicals and byproducts formed in industrial or combustion processes have been, and still are, released in the environment. The toxic matter is known to adversely impact the ecosystem as well as human life. The increasing number of potentially harmful pollutants in the environment has resulted in stringent legislation towards environmental regulation. In order to implement these tight regulations, analytical instruments that can provide high sensitivity, accurate and instantaneous results in a form factor that can be deployed in remote 
locations are critical. There is a critical need to monitor contaminants and toxins in air (volatile compounds and gases), water (heavy metals, virus and bacteria), soil (chemicals, minerals) and food (bacteria, viruses, toxins, mycotoxins, pyrethroids, etc.). Where online and continuous monitoring of environmental variable is imperative, traditional laboratory based analytical methods will not suffice. Point of care systems driven by microfluidic technology is a key player in the realization of such environmental sensors. Unlike biomedical applications where sterility and biocompatibility are critical issues considered during development, design criteria is mostly based on robustness and performance in harsh environments. Packaging of the sensors to withstand harsh environmental conditions is crucial, which is where, the use of LTCC based microfluidic systems can provide significant advantages over most conventional microfluidic systems, such as mature packaging techniques, inertness to harsh chemicals and gases and noncorrosiveness.

Achmann et al. [177] have reported the development of an LTCC based microfluidic gas sensing system for the detection of formaldehyde. A biosensors approach, where formaldehyde dehydrogenase, an enzyme, is used as the sensing element in an amperometric sensing set-up. In order to support system integration, a ceramic diffusion membrane has been incorporated into the LTCC stack. The typical hydrophobicity required in the diffusion membrane is achieved by coating the ceramic membrane with an organochlorosilane in toluene. The sensing electrodes are realized by standard screen printing of $\mathrm{Ag} / \mathrm{AgCl}$. The sensor exhibits a low detection limit of $76 \mathrm{ppb}$ with linear response in the range $500 \mathrm{ppb}$ to $10 \mathrm{ppm}$. The sensor performance is comparable to a macro sized counterpart while requiring half the enzymes and one tenth 
the sample size. In an effort to develop a deployable on-site microanalyzer, Garcia et al. have [178] developed an LTCC microfluidic system for the simultaneous detection of nitrate and chloride ions in water samples. Two set of screen printed $\mathrm{Ag} / \mathrm{AgCl}$ electrodes are used for potentiometric detection, and ion selective polymer membranes are used for selective detection of chlorine and nitrate ions. The integrated sensor performance demonstrated a linear working range of $10^{-3} \mathrm{M}$ to $10^{-1} \mathrm{M}$ for nitrate ions and $10^{-4} \mathrm{M}$ to $10^{-}$ ${ }^{1} \mathrm{M}$ for chloride ion. Similarly, amperometric determination of free chlorine using carbon nanotube composite electrodes has been reported[179]. The use of nanocomposites increases the system functionality and also enhances the surface renewability inherent in composite electrodes. A noticeably lower detection limit of $0.05 \mathrm{mg} / \mathrm{L}$ of chlorine ion was detected. Real samples collected from swimming pools were successfully tested for determination of chlorine using the developed LTCC based sensing system. Contamination of water bodies with metallic particulates originating as byproducts from industrial processes is a cause for serious concern. The presence of metallic contaminants in potable water has been correlated to many cases of carcinogenesis. An LTCC based spectrophotometric microanalysis system [180] has been developed for the determination of chromium (VI) in water. The microfluidic system consists of inlet and outlet microchannels that transport the sample solution into and out of the measurement chamber. A glass window incorporated into the opaque LTCC structure allows the use of optical detection system such as colorimetry for determining the concentration of the target analyte. The developed system provides a linear sensing range of 0.1 to $20 \mathrm{mg} / \mathrm{L}$ at a detection limit of $50 \mu \mathrm{g} / \mathrm{L}$. An LTCC based microfluidic system for detecting traces of heavy metals in biological and environmental fluids has been reported by Rubio et al 
[181]. The design features of the LTCC microfluidic manifold include a spiral coil microchannel for passive mixing of two liquids required in most electro-chemical sensing assays and a screen printed microelectrode array that is required for the electrochemical detection technique. An important part of the chip fabrication is the precise alignment of the electrode array with the microchannels. In this work, an innovative approach has been utilized; where in, a standard dental $\mathrm{x}$-ray image is used to verify the alignment, since visual alignment is not possible in the opaque structure. The integrated system demonstrates detection of mercury and copper in low detection limits of $0.9 \mu \mathrm{g} / \mathrm{L}$ and $0.45 \mu \mathrm{g} / \mathrm{L}$ respectively in urinary fluids. The same system can be employed for detection in environmental fluids such as river water and water bodies around industrial sites. For the detection of organic compounds such as sulfamethoxazole (SMX) and trimethoprim (TMP), Almeida et al. [182] have developed an LTCC- potentiometric microfluidic device. SMX, which has been used for many years as a human/veterinary antibiotic is now a potential threat as it is found in alarming quantities in water coming from municipal wastewaters and aqua farming water bodies. The developed sensing system uses synthesized antibodies as the sensing material. Molecular imprinting technique has been used to create ionophores of SMX on a plasticized PVC membrane. The membrane is then integrated into the LTCC microfluidic manifold for detection of SMX and similarly TMP. The sensor displays slopes of $-58.7 \mathrm{mV} /$ decade for a detection range from $12.7 \mu \mathrm{g} / \mathrm{mL}$ to $250 \mu \mathrm{g} / \mathrm{mL}$ at a detection limit of $3.85 \mu \mathrm{g} / \mathrm{mL}$ at a high throughput of 36 samples/h. The sensor utilizes a sample volume of $3.3 \mathrm{~mL}$ per sample.

Various examples of systems application of LTCC based microfluidic devices have been reviewed. However, there remain many opportunities for further development 
of the processing techniques of LTCC to realize novel functionality and applications. Realization of feature sizes in the nanoscale range in LTCC systems is currently limited by the fabrication techniques. Novel fabrication techniques along with the use of new material compositions can enable the creation of nanoscale features such as nanochannels, nanowires and other nanotextures surfaces. Methods to integrate LTCC with silicon, glass, and polymers can lead to the creation of hybrid microfluidic devices that bring together the best from all the materials. LTCC microfluidic systems with actuators for microvalves and micro pumps will be essential for realization of a standalone point of care system. 


\subsection{References}

1. Corbalán-Tutau, M.D., et al., Daily profile in two circadian markers "melatonin and cortisol" and associations with Metabolic Syndrome components. Physiology \& Behavior, 2012.

2. Holsboer, F. and M. Ising, Stress hormone regulation: biological role and translation into therapy. Annual review of psychology, 2010. 61: p. 81-109.

3. McEwen, B.S., Cortisol, Cushing's Syndrome, and a Shrinking Brain-New Evidence for Reversibility. Journal of Clinical Endocrinology \& Metabolism, 2002. 87(5): p. 1947-1948.

4. Ross, I., et al., Salivary Cortisol Day Curves in Addison's Disease in Patients on Hydrocortisone Replacement. Hormone and Metabolic Research, 2012(EFirst).

5. Brossaud, J., et al. Urinary cortisol metabolites in corticotroph and adrenal tumours. in Endocrine Abstracts. 2012.

6. Venugopal, M., et al., Clinical Evaluation of a Novel Interstitial Fluid Sensor System for Remote Continuous Alcohol Monitoring. Sensors Journal, IEEE, 2008. 8(1): p. 71-80.

7. Venugopal, M., et al., A realtime and continuous assessment of cortisol in ISF using electrochemical impedance spectroscopy. Sensors and Actuators A: Physical, 2011. 172(1): p. 154-160.

8. El-Laboudi, A., et al., Use of Microneedle Array Devices for Continuous Glucose Monitoring: A Review. Diabetes Technology \& Therapeutics, 2013. 15(1): p. 101115.

9. $\quad$ Prausnitz, M.R., Microneedles for transdermal drug delivery. Advanced Drug Delivery Reviews, 2004. 56(5): p. 581-587.

10. Khanna, P., et al., Microneedle-Based Automated Therapy for Diabetes Mellitus. Journal of Diabetes Science Technology, 2008. 2(6): p. 1122-1129.

11. Mukerjee, E.V., et al., Microneedle array for transdermal biological fluid extraction and in situ analysis. Sensors and Actuators A: Physical, 2004. 114(23): p. 267-275.

12. Wang, P.M., M. Cornwell, and M.R. Prasunitz, Minimally Invasive Extraction of Dermal Interstitial Fluid for Glucose Monitoring Using Microneedles. Diabetes Technology \& Therapeutics, 2005. 7(1): p. 131-141. 
13. Bennett, A. and V. Hayssen, Hair as a biological indicator of drug use, drug abuse or chronic exposure to environmental toxicants. International Journal of Toxicology, 2010. 25: p. 143-163.

14. Koren, L., et al., A novel method using hair for determining hormonal levels in wildlife. Animal Behaviour, 2002. 63(2): p. 403-406.

15. Sauve, B., et al., Measurement of cortisol in human hair as a biomarker of systemic exposure. Clinical and Investigative Medicine, 2007. 30(5): p. 83-91.

16. Gao, W., et al., HPLC-FLU detection of cortisol distribution in human hair. Clinical Biochemistry, 2010. 43(7-8): p. 677-682.

17. Manenschijn, L., et al., Evaluation of a method to measure long term cortisol levels. Steroids, 2011. 76(10-11): p. 1032-1036.

18. Raul, J.-S., et al., Detection of physiological concentrations of cortisol and cortisone in human hair. Clinical Biochemistry, 2004. 37(12): p. 1105-1111.

19. Stalder, T., et al., Cortisol in hair, body mass index and stress-related measures. Biological Psychology, 2012. 90(3): p. 218-223.

20. Gow, R., et al., An assessment of cortisol analysis in hair and its clinical applications. Forensic Science International, 2010. 196(1-3): p. 32-37.

21. Kalra, S., et al., The relationship between stress and hair cortisol in healthy pregnant women. Clinical and Investigative Medicine 2007. 30(2): p. 103-107.

22. Krischbaum, C., et al., Hair as a retrospective calendar of cortisol productionIncreased cortisol incorporation into hair in the third trimester of pregnancy. Psychoneuroendocrinology, 2009. 34(1): p. 32-37.

23. Prunty, H., et al. Sweat patch cortisol-a new screen for Cushing's syndrome. in Endocrine Abstracts. 2004.

24. Russell, E., et al., The Detection of Cortisol in Human Sweat: Implications for Measurement of Cortisol in Hair. Endocrine Reviews, 2012. 33(03).

25. Le Roux, C., et al., Free cortisol index is better than serum total cortisol in determining hypothalamic-pituitary-adrenal status in patients undergoing surgery. Journal of Clinical Endocrinology \& Metabolism, 2003. 88(5): p. 20452048.

26. Levine, A., et al., Measuring cortisol in human psychobiological studies. Physiology \& Behavior, 2007. 90(1): p. 43-53. 
27. VanBruggen, M., et al., The relationship between serum and salivary cortisol levels in response to different intensities of exercise. International journal of sports physiology and performance, 2011. 6(3): p. 396.

28. Teruhisa, U., et al., Use of saliva for monitoring unbound free cortisol levels in serum. Clinica Chimica Acta, 1981. 110(2): p. 245-253.

29. Van Caenegem, E., et al. Salivary cortisol and testosterone: a comparison of salivary sample collection methods in healthy controls. in Endocrine Abstracts. 2011.

30. Price, D., G. Close, and B. Fielding, Age of appearance of circadian rhythm in salivary cortisol values in infancy. Archives of Disease in Childhood, 1983. 58(6): p. 454-456.

31. Raff, H., J.L. Raff, and J.W. Findling, Late-night salivary cortisol as a screening test for Cushing's syndrome. Journal of Clinical Endocrinology \& Metabolism, 1998. 83(8): p. 2681-2686.

32. Løvås, K., T. Thorsen, and E. Husebye, Saliva cortisol measurement: simple and reliable assessment of the glucocorticoid replacement therapy in Addison's disease. Journal of endocrinological investigation, 2006. 29(8): p. 727.

33. Granger, A., et al., Effects of Physical Stress and Maturational Changes on Hypothalamic Pituitary Adrenal Axis Function Through Cortisol Analysis. 2012.

34. Carpenter, L.L., et al., Effect of childhood physical abuse on cortisol stress response. Psychopharmacology, 2011. 214(1): p. 367-375.

35. Turpeinen, U., et al., Determination of urinary free cortisol by HPLC. Clinical chemistry, 1997. 43(8): p. 1386-1391.

36. Abraham, G.E., J.E. Buster, and R.C. Teller, Radioimmunoassay of plasma cortisol. Analytical Letters, 1972. 5(11): p. 757-765.

37. RUDER, H.J., R.L. GUY, and M.B. LIPSETT, A radioimmunoassay for cortisol in plasma and urine. Journal of Clinical Endocrinology \& Metabolism, 1972. 35(2): p. 219-224.

38. Kao, M., et al., Parallel radioimmunoassay for plasma cortisol and 11deoxycortisol. Clinical chemistry, 1975. 21(11): p. 1644-1647.

39. Dash, R., et al., A specific, non-chromatographic radioimmunoassay for human plasma cortisol. Steroids, 1975. 26(5): p. 647-661. 
40. Gomez-Sanchez, C., L. Milewich, and O.B. Holland, Radioiodinated derivatives for steroid radioimmunoassay. Application to the radioimmunoassay of cortisol. J. Lab. Clin. Med.;(United States), 1977. 89(4).

41. Kobayashi, Y., et al., Fluorescence polarization immunoassay for cortisol. Clinica Chimica Acta, 1979. 92(2): p. 241-247.

42. Appel, D., et al., A fluorimetric assay for cortisol. Analytical and Bioanalytical Chemistry, 2005. 383(2): p. 182-186.

43. Carrozza, C., et al., Clinical accuracy of midnight salivary cortisol measured by automated electrochemiluminescence immunoassay method in Cushing's syndrome. Annals of clinical biochemistry, 2010. 47(3): p. 228-232.

44. Yaneva, M., G. Kirilov, and S. Zacharieva, Midnight salivary cortisol, measured by highly sensitive electrochemiluminescence immunoassay, for the diagnosis of Cushing's syndrome. Central European Journal of Medicine, 2009. 4(1): p. 59-64.

45. Belaya, Z.E., et al., Diagnostic performance of late-night salivary cortisol measured by automated electrochemiluminescence immunoassay in obese and overweight patients referred to exclude Cushing's syndrome. Endocrine, 2012: p. $1-7$.

46. Lippi, G., et al., Measurement of morning saliva cortisol in athletes. Clinical Biochemistry, 2009. 42(9): p. 904-906.

47. Pervanidou, P., et al., The natural history of neuroendocrine changes in pediatric posttraumatic stress disorder (PTSD) after motor vehicle accidents: Progressive divergence of noradrenaline and cortisol concentrations over time. Biological Psychiatry, 2007. 62(10): p. 1095-1102.

48. McRae, A.L., et al., Stress reactivity: biological and subjective responses to the cold pressor and Trier Social stressors. Human Psychopharmacology: Clinical and Experimental, 2006. 21(6): p. 377-385.

49. Lewis, J. and P. Elder, An enzyme-linked immunosorbent assay (ELISA) for plasma cortisol. Journal of steroid biochemistry, 1985. 22(5): p. 673-676.

50. Shimada, M., et al., Determination of salivary cortisol by ELISA and its application to the assessment of the circadian rhythm in children. Hormone Research in Paediatrics, 1995. 44(5): p. 213-217.

51. Small, B.C. and K.B. Davis, Validation of a Time-Resolved Fluoroimmunoassay for Measuring Plasma Cortisol in Channel Catfish Ictalurus punctatus. Journal of 
the World Aquaculture Society, 2007. 33(2): p. 184-187.

52. Cooper, M.A., Label-free screening of bio-molecular interactions. Analytical and Bioanalytical Chemistry, 2003. 377(5): p. 834-842.

53. Shankaran, D.R., K.V. Gobi, and N. Miura, Recent advancements in surface plasmon resonance immunosensors for detection of small molecules of biomedical, food and environmental interest. Sensors and Actuators B: Chemical, 2007. 121(1): p. 158-177.

54. Mitchell, J.S., T.E. Lowe, and J.R. Ingram, Rapid ultrasensitive measurement of salivary cortisol using nano-linker chemistry coupled with surface plasmon resonance detection. Analyst, 2008. 134(2): p. 380-386.

55. Stevens, R.C., et al., Detection of cortisol in saliva with a flow-filtered, portable surface plasmon resonance biosensor system. Analytical Chemistry, 2008. 80(17): p. 6747-6751.

56. Atashbar, M.Z., et al., QCM biosensor with ultra thin polymer film. Sensors and Actuators B: Chemical, 2005. 107(2): p. 945-951.

57. Purvis, D., et al., An ultrasensitive and stable potentiometric immunosensor. Biosensors and Bioelectronics, 2003. 18(11): p. 1385-1390.

58. Ni, Y., Y. Wang, and S. Kokot, Simultaneous determination of three fluoroquinolones by linear sweep stripping voltammetry with the aid of chemometrics. Talanta, 2006. 69(1): p. 216-225.

59. Nicholson, R.S., Theory and Application of Cyclic Voltammetry for Measurement of Electrode Reaction Kinetics. Analytical Chemistry, 1965. 37(11): p. 13511355.

60. MacLeod, A.J., A note on the Randles-Sevcik function from electrochemistry. Applied mathematics and computation, 1993. 57(2): p. 305-310.

61. Sun, K., N. Ramgir, and S. Bhansali, An immunoelectrochemical sensor for salivary cortisol measurement. Sensors and Actuators B: Chemical, 2008. 133(2): p. 533-537.

62. Vasudev, A., et al., An LTCC-based microfluidic system for label-free, electrochemical detection of cortisol. Sensors and Actuators B: Chemical, 2013.

63. Arya, S.K., A. Dey, and S. Bhansali, Polyaniline protected gold nanoparticles based mediator and label free electrochemical cortisol biosensor. Biosensors and Bioelectronics, 2011. 28(1): p. 166-173. 
64. Moreno-Guzmán, M., et al., Disposable immunosensor for cortisol using functionalized magnetic particles. Analyst, 2010. 135(8): p. 1926-1933.

65. Katz, E. and I. Willner, Probing Biomolecular Interactions at Conductive and Semiconductive Surfaces by Impedance Spectroscopy: Routes to Impedimetric Immunosensors, DNA-Sensors, and Enzyme Biosensors. Electroanalysis, 2003. 15(11): p. 913-947.

66. Chang, B.-Y. and S.-M. Park, Electrochemical impedance spectroscopy. Annual Review of Analytical Chemistry, 2010. 3: p. 207-229.

67. Yang, L., Y. Li, and G.F. Erf, Interdigitated Array Microelectrode-Based Electrochemical Impedance Immunosensor for Detection of Escherichia c oli O157: H7. Analytical Chemistry, 2004. 76(4): p. 1107-1113.

68. La Belle, J.T., et al., A cytokine immunosensor for multiple sclerosis detection based upon label-free electrochemical impedance spectroscopy. Biosensors and Bioelectronics, 2007. 23(3): p. 428-431.

69. Hnaien, M., et al., Immobilization of specific antibody on SAM functionalized gold electrode for rabies virus detection by electrochemical impedance spectroscopy. Biochemical Engineering Journal, 2008. 39(3): p. 443-449.

70. Zhuo, Y., et al., A reagentless amperometric immunosensor based on gold nanoparticles/thionine/Nafion-membrane-modified gold electrode for determination of $\alpha$-1-fetoprotein. Electrochemistry Communications, 2005. 7(4): p. 355-360.

71. Wu, C.-C., C.-H. Lin, and W.-S. Wang, Development of an enrofloxacin immunosensor based on label-free electrochemical impedance spectroscopy. Talanta, 2009. 79(1): p. 62-67.

72. Arya, S.K., et al., Dithiobis (succinimidyl propionate) modified gold microarray electrode based electrochemical immunosensor for ultrasensitive detection of cortisol. Biosensors and Bioelectronics, 2010. 25(10): p. 2296-2301.

73. Arya, S.K., et al., Antibody modified gold micro array electrode based electrochemical immunosensor for ultrasensitive detection of cortisol in saliva and ISF. Procedia Engineering, 2010. 5: p. 804-807.

74. Caruso, F., Nanoengineering of Particle Surfaces. Advanced Materials, 2001. 13(1): p. 11-22.

75. Nabok, A., Organic and inorganic nanostructures. Vol. 8. 2005: Artech House. 
76. Wang, J., Nanomaterial-based electrochemical biosensors. Analyst, 2005. 130(4): p. 421-426.

77. Wang, J., Nanomaterial-based amplified transduction of biomolecular interactions. Small, 2005. 1(11): p. 1036-1043.

78. Elisabetta, C., Metal oxide nano-crystals for gas sensing. Analytica Chimica Acta, 2006. 568(1-2): p. 28-40.

79. Khatko, V., et al., New technology of metal oxide thin film preparation for chemical sensor application. Sensors and Actuators B: Chemical, 2005. 109(1): p. 128-134.

80. Barsan, N., D. Koziej, and U. Weimar, Metal oxide-based gas sensor research: How to? Sensors and Actuators B: Chemical, 2007. 121(1): p. 18-35.

81. Tan, O.K., et al., Nano-structured oxide semiconductor materials for gas-sensing applications. Ceramics International, 2004. 30(7): p. 1127-1133.

82. Tan, O.K., et al., Nanostructured oxides by high-energy ball milling technique: application as gas sensing materials. Solid State Ionics, 2004. 172(1-4): p. 309316.

83. Barsan, N. and U. Weimar, Conduction Model of Metal Oxide Gas Sensors. Journal of Electroceramics, 2001. 7(3): p. 143-167.

84. Carotenuto, G., et al., Metal-Polymer Nanocomposite Synthesis: Novel ex situ and in situ Approaches, in Metal-Polymer Nanocomposites. 2005, John Wiley \& Sons, Inc. p. 155-181.

85. Wang, C., et al., Metal Oxide Gas Sensors: Sensitivity and Influencing Factors. Sensors, 2010. 10(3): p. 2088-2106.

86. Fine, G.F., et al., Metal Oxide Semi-Conductor Gas Sensors in Environmental Monitoring. Sensors, 2010. 10(6): p. 5469-5502.

87. Saxena, V. and B.D. Malhotra, Prospects of conducting polymers in molecular electronics. Current Applied Physics, 2003. 3(2-3): p. 293-305.

88. MacDiarmid, A.G., "Synthetic Metals": A Novel Role for Organic Polymers (Nobel Lecture). Angewandte Chemie International Edition, 2001. 40(14): p. 2581-2590.

89. Liu, T., C. Burger, and B. Chu, Nanofabrication in polymer matrices. Progress in 
Polymer Science, 2003. 28(1): p. 5-26.

90. Inzelt, G., Applications of Conducting Polymers Conducting Polymers. 2012, Springer Berlin Heidelberg. p. 245-293.

91. Korotcenkov, G., Chemical Sensors Fundamentals of Sensing Materials: Volume 3 Polymers and Other Materials. 2011: Momentum Press.

92. Adhikari, B. and S. Majumdar, Polymers in sensor applications. Progress in Polymer Science, 2004. 29(7): p. 699-766.

93. Barisci, J.N., et al., Conducting polymer sensors for monitoring aromatic hydrocarbons using an electronic nose. Sensors and Actuators B: Chemical, 2002. 84(2-3): p. 252-257.

94. Gangopadhyay, R. and A. De, Conducting polymer composites: novel materials for gas sensing. Sensors and Actuators B: Chemical, 2001. 77(1-2): p. 326-329.

95. de Melo, C.P., et al., Use of conducting polypyrrole blends as gas sensors. Sensors and Actuators B: Chemical, 2005. 109(2): p. 348-354.

96. Zeng, W., et al., Conductive polymer composites as gas sensors with size-related molecular discrimination capability. Sensors and Actuators B: Chemical, 2007. 124(1): p. 118-126.

97. Blair, R., et al., Construction of a Polyaniline Nanofiber Gas Sensor. Journal of Chemical Education, 2008. 85(8): p. 1102.

98. Xie, D., et al., Fabrication and characterization of polyaniline-based gas sensor by ultra-thin film technology. Sensors and Actuators B: Chemical, 2002. 81(2-3): p. $158-164$.

99. Jun, H.-K., et al., Electrical properties of polypyrrole gas sensors fabricated under various pretreatment conditions. Sensors and Actuators B: Chemical, 2003. 96(3): p. 576-581.

100. Chang, J.B., et al., Printable polythiophene gas sensor array for low-cost electronic noses. Journal of Applied Physics, 2006. 100(1): p. 014506-7.

101. Jung, Y.S., et al., Nanowire Conductive Polymer Gas Sensor Patterned Using Self-Assembled Block Copolymer Lithography. Nano Letters, 2008. 8(11): p. 3776-3780.

102. Janata, J. and M. Josowicz, Conducting polymers in electronic chemical sensors. Nat Mater, 2003. 2(1): p. 19-24. 
103. Hou, K.-Y., A. Rehman, and X. Zeng, Study of Ionic Liquid Immobilization on Polyvinyl Ferrocene Substrates for Gas Sensor Arrays. Langmuir, 2011. 27(8): p. 5136-5146.

104. Kaushik, A., et al., Hybrid Cross-Linked Polyaniline-WO3 Nanocomposite Thin Film for NOx Gas Sensing. Journal of Nanoscience and Nanotechnology, 2009. 9(3): p. 1792-1796.

105. Nastase, C., et al., Nanocomposites based on functionalized nanotubes in polyaniline matrix by plasma polymerization. Progress in Solid State Chemistry, 2006. 34(2-4): p. 181-189.

106. Gómez-Romero, P., et al., Hybrid organic-inorganic nanocomposite materials for application in solid state electrochemical supercapacitors. Electrochemistry Communications, 2003.5(2): p. 149-153.

107. Shi, J.-1., Z.-1. Hua, and L.-x. Zhang, Nanocomposites from ordered mesoporous materials. Journal of Materials Chemistry, 2004. 14(5): p. 795-806.

108. Shchipunov, Y.A., T.Y.Y. Karpenko, and A.V. Krekoten, Hybrid organicinorganic nanocomposites fabricated with a novel biocompatible precursor using sol-gel processing. Composite Interfaces, 2005. 11(8-9): p. 587-607.

109. Kaushik, A., et al., Fabrication and Characterization of PolyanilineZnO Hybrid Nanocomposite Thin Films. Journal of Nanoscience and Nanotechnology, 2008. 8(4): p. 1757-1761.

110. Burghard, Z., et al., Nanomechanical Properties of Bioinspired OrganicInorganic Composite Films. Advanced Materials, 2007. 19(7): p. 970-974.

111. Godovsky, D., Device Applications of Polymer-Nanocomposites Biopolymers · PVA Hydrogels, Anionic Polymerisation Nanocomposites. 2000, Springer Berlin / Heidelberg. p. 163-205.

112. Hatchett, D.W. and M. Josowicz, Composites of Intrinsically Conducting Polymers as Sensing Nanomaterials. Chemical Reviews, 2008. 108(2): p. 746769.

113. Guido, K., Concepts for the incorporation of inorganic building blocks into organic polymers on a nanoscale. Progress in Polymer Science, 2003. 28(1): p. 83-114.

114. Portney, N.G., et al., Organic and Inorganic Nanoparticle Hybrids. Langmuir, 2005. 21(6): p. 2098-2103. 
115. Rozenberg, B.A. and R. Tenne, Polymer-assisted fabrication of nanoparticles and nanocomposites. Progress in Polymer Science, 2008. 33(1): p. 40-112.

116. Kruk, M. and M. Jaroniec, Gas Adsorption Characterization of Ordered Organic-Inorganic Nanocomposite Materials. Chemistry of Materials, 2001. 13(10): p. 3169-3183.

117. Mitzi, D.B., Thin-Film Deposition of Organic-Inorganic Hybrid Materials. Chemistry of Materials, 2001. 13(10): p. 3283-3298.

118. Sukpirom, N. and M.M. Lerner, Preparation of Organic-Inorganic Nanocomposites with a Layered Titanate. Chemistry of Materials, 2001. 13(6): p. 2179-2185.

119. Vivekchand, S.R.C., et al., Electrical properties of inorganic nanowire-polymer composites. Journal of Materials Chemistry, 2005. 15(46): p. 4922-4927.

120. Mitzi, D.B., K. Chondroudis, and C.R. Kagan, Organic-inorganic electronics. IBM Journal of Research and Development, 2001. 45(1): p. 29-45.

121. Whitesides, G.M., The origins and the future of microfluidics. Nature, 2006. 442(7101): p. 368-373.

122. Wu, Z. and S. Cheng, Microfluidic electronics. Lab on a Chip, 2012.

123. Squires, T.M. and S.R. Quake, Microfluidics: Fluid physics at the nanoliter scale. Reviews of Modern Physics, 2005. 77(3): p. 977-1026.

124. Barry, R. and D. Ivanov, Microfluidics in biotechnology. Journal of Nanobiotechnology, 2004. 2(1): p. 2.

125. Psaltis, D., S.R. Quake, and C. Yang, Developing optofluidic technology through the fusion of microfluidics and optics. Nature, 2006. 442(7101): p. 381-386.

126. Marre, S., Y. Roig, and C. Aymonier, Supercritical microfluidics: Opportunities in flow-through chemistry and materials science. The Journal of Supercritical Fluids, (0).

127. Jiang Zhe, A.J., Prashanta Dutta, Jun Hu and Joan Carletta, A micromachined high throughput Coulter counter for bioparticle detection and counting. Journal of Micromechanics and Microengineering, 2007. 17(2): p. 304-313.

128. Zaytseva, N.V., et al., Development of a microfluidic biosensor module for pathogen detection. Lab on a Chip, 2005. 5(8): p. 805-811. 
129. Zhang, C., et al., PCR microfluidic devices for DNA amplification. Biotechnology Advances, 2006. 24(3): p. 243-284.

130. Zappe, A.W.a.H., Tunable microfluidic microlenses. Applied Optics, 2005. 44(16): p. 3238-3245.

131. Choban, E.R., et al., Microfluidic fuel cell based on laminar flow. Journal of Power Sources, 2004. 128(1): p. 54-60.

132. Linder, V., Microfluidics at the crossroad with point-of-care diagnostics. Analyst, 2007. 132(12): p. 1186-1192.

133. Sista, R., et al., Development of a digital microfluidic platform for point of care testing. Lab on a Chip, 2008. 8(12): p. 2091-2104.

134. Gervais, L., N. de Rooij, and E. Delamarche, Microfluidic Chips for Point-ofCare Immunodiagnostics. Advanced Materials, 2011. 23(24): p. H151-H176.

135. Delattre, C., et al., Macro to microfluidics system for biological environmental monitoring. Biosensors and Bioelectronics, 2012. 36(1): p. 230-235.

136. Anderson, J.R., et al., Fabrication of Topologically Complex Three-Dimensional Microfluidic Systems in PDMS by Rapid Prototyping. Analytical Chemistry, 2000. 72(14): p. 3158-3164.

137. Becker, H. and L.E. Locascio, Polymer microfluidic devices. Talanta, 2002. 56(2): p. 267-287.

138. Ibáñez-García, N., et al., Green-tape ceramics. New technological approach for integrating electronics and fluidics in microsystems. TrAC Trends in Analytical Chemistry, 2008. 27(1): p. 24-33.

139. Gongora-Rubio, M.R., et al., Overview of low temperature co-fired ceramics tape technology for meso-system technology (MSST). Sensors and Actuators A: Physical, 2001. 89(3): p. 222-241.

140. Peterson, K.A., et al., Novel Microsystem Applications with New Techniques in Low-Temperature Co-Fired Ceramics. International Journal of Applied Ceramic Technology, 2005. 2(5): p. 345-363.

141. Golonka, L.J., et al., LTCC Microfluidic System. International Journal of Applied Ceramic Technology, 2006. 3(2): p. 150-156.

142. Leszek Golonka, P.B., Dominik Jurków, Karol Malecha, HEnryk Roguszczak, 
Rafał Tadaszak, Low temperature co-fired ceramics (LTCC) microsysttems. Optica Applicata, 2011. 41(2): p. 383-388.

143. GOLONKA, L.J., Technology and applications of Low Temperature Cofired Ceramic (LTCC) based sensors and microsystems. BULLETIN OF THE POLISH ACADEMY OF SCIENCES: TECHNICAL SCIENCES, 2006. 54(2).

144. W. Kinzy Jones, Y.L., Brooks Larsen, Peng Wang, and Marc Zampino, Chemical, Structural, and Mechanical Properties of the LTCC Tapes. The International Journal of Microcircuits and Electronic Packaging, 2000. 23(4): p. 469-473.

145. Shafique, M.F. and I.D. Robertson. Rapid prototyping of LTCC microwave circuits using laser machining. in Microwave Symposium Digest, 2009. MTT '09. IEEE MTT-S International. 2009.

146. Ramos, F.M., et al. Monolithic Ceramic Technology for Sensing Devices. in Electron Devices, 2009. CDE 2009. Spanish Conference on. 2009.

147. Smetana, W., et al., Processing procedures for the realization of fine structured channel arrays and bridging elements by LTCC-Technology. Microelectronics Reliability, 2009. 49(6): p. 592-599.

148. Birol, H., T. Maeder, and P. Ryser, Application of graphite-based sacrificial layers for fabrication of LTCC (low temperature co-fired ceramic) membranes and micro-channels. Journal of Micromechanics and Microengineering, 2007. 17(1): p. 50.

149. Malecha, K. and L.J. Golonka, Microchannel fabrication process in LTCC ceramics. Microelectronics Reliability, 2008. 48(6): p. 866-871.

150. Malecha, K. and L.J. Golonka, Three-dimensional structuration of zero-shrinkage LTCC ceramics for microfluidic applications. Microelectronics Reliability, 2009. 49(6): p. 585-591.

151. Farhan Shafique, M., et al., Fabrication of embedded microfluidic channels in low temperature co-fired ceramic technology using laser machining and progressive lamination. Journal of the European Ceramic Society, 2011. 31(13): p. 21992204.

152. Ulrike Deisinger, T.F., Andreas Roosen. Realisation of Large Cavities in Multilayer Ceramics by Cold Low Pressure Lamination and Their Characterization by $m C T$. in IMAPS/ACerS 8th International CICMT Conference and Exhibition. 2012. Erfurt, Germany.

153. Thomas Maeder, B.J., Fabrizio Vecchio, Caroline Jacq, Peter Ryser, Paul Muralt. 
Lamination of LTCC at low pressure and moderate temperature using screenprinted adhesives. in IMAPS/ACerS 8th International CICMT Conference and Exhibition. 2012. Erfurt, Germany.

154. Malecha, K., I. Gancarz, and L.J. Golonka, A PDMS/LTCC bonding technique for microfluidic application. Journal of Micromechanics and Microengineering, 2009. 19(10): p. 105016.

155. Rusu, C., et al., LTCC interconnects in microsystems. Journal of Micromechanics and Microengineering, 2006. 16(6): p. S13.

156. Eddings, M.A., M.A. Johnson, and B.K. Gale, Determining the optimal PDMSPDMS bonding technique for microfluidic devices. Journal of Micromechanics and Microengineering, 2008. 18(6): p. 067001.

157. Bembnowicz, P. and L.J. Golonka, Integration of transparent glass window with LTCC technology for $\mu$ TAS application. Journal of the European Ceramic Society, 2010. 30(3): p. 743-749.

158. Karol Malecha, L.J.G., Jerzy Bałdyga, Magdalena Jasin' ska, Paweł Sobieszuk, Serpentine microfluidic mixer made in LTCC. Sensors and Actuators B: Chemical, 2009. 143: p. 400-413.

159. Schlottig, G., et al. Modeling LTCC-based Microchannels Using a Network Approach. in Electronics Systemintegration Technology Conference, 2006. 1st. 2006.

160. Malecha, K. and L.J. Golonka. CFD simulations of LTCC based microsystems. in Electronics Technology, 2006. ISSE '06. 29th International Spring Seminar on. 2006.

161. G.A. Groß, T.T., S. Schneider, D. Boskovic, J.M. Köhler, Fabrication and fluidic characterization of static micromixers made of low temperature cofired ceramic (LTCC). Chemical Engineering Science, 2008. 63: p. 2773-2784.

162. Roberge, D.M., et al., Microreactor Technology: A Revolution for the Fine Chemical and Pharmaceutical Industries? Chemical Engineering \& Technology, 2005. 28(3): p. 318-323.

163. Yang, J., et al., High sensitivity PCR assay in plastic micro reactors. Lab on a Chip, 2002. 2(4): p. 179-187.

164. DeWitt, S.H., Micro reactors for chemical synthesis. Current Opinion in Chemical Biology, 1999. 3(3): p. 350-356. 
165. Malodobra, M., et al., The specificity, sensitivity and efficiency of the PCR microsystem based on LTCC technology, in Proceedings of the 11th WSEAS international conference on Applied informatics and communications, and Proceedings of the 4th WSEAS International conference on Biomedical electronics and biomedical informatics, and Proceedings of the international conference on Computational engineering in systems applications. 2011, World Scientific and Engineering Academy and Society (WSEAS): Florence, Italy. p. 327-331.

166. Bembnowicz, P., et al., Preliminary studies on LTCC based PCR microreactor. Sensors and Actuators B: Chemical, 2010. 150(2): p. 715-721.

167. Pawel Bembnowicz, P.H., Malgorzata Malodobra, Anna Karpiewska, Leszek J. Golonka, Anna Jonkisz, Tadeusz Dobosz, The low temperature co-fired ceramics (LTCC) chip for polymerase chain reaction (PCR) application. Optica Applicata, 2011. 41(2): p. 10.

168. Sadler, D.J., et al., Thermal management of BioMEMS: temperature control for ceramic-based PCR and DNA detection devices. Components and Packaging Technologies, IEEE Transactions on, 2003. 26(2): p. 309-316.

169. Smetana, W., et al., A Ceramic Microfluidic Device for Monitoring Complex Biochemical Reactive Systems Biomedical Engineering Systems and Technologies, A. Fred, J. Filipe, and H. Gamboa, Editors. 2010, Springer Berlin Heidelberg. p. 110-123.

170. Smetana, W., et al., A multi-sensor biological monitoring module built up in LTCC-technology. Microelectronic Engineering, 2007. 84(5-8): p. 1240-1243.

171. Ciosek, P., et al., Monitoring of cell cultures with LTCC microelectrode array. Analytical and Bioanalytical Chemistry, 2009. 393(8): p. 2029-2038.

172. Malecha, K., et al., Micro Ceramic Cell Analyzer (MCCA) - Preliminary results. Microelectronics Reliability, 2011.51(7): p. 1250-1252.

173. Fakunle, E.S., et al., Evaluation of Screen-Printed Gold on Low-Temperature CoFired Ceramic as a Substrate for the Immobilization of Electrochemical Immunoassays $\dagger$. Langmuir, 2006. 22(25): p. 10844-10853.

174. Fakunle, E.S. and I. Fritsch, Low-temperature co-fired ceramic microchannels with individually addressable screen-printed gold electrodes on four walls for self-contained electrochemical immunoassays. Analytical and Bioanalytical Chemistry, 2010. 398(6): p. 2605-2615.

175. Malecha, K., et al., LTCC microreactor for urea determination in biological 
fluids. Sensors and Actuators B: Chemical, 2009. 141(1): p. 301-308.

176. Malecha, K., et al., Low temperature co-fired ceramic (LTCC)-based biosensor for continuous glucose monitoring. Sensors and Actuators B: Chemical, 2011. 155(2): p. 923-929.

177. Achmann, S., et al., Miniaturized low temperature co-fired ceramics (LTCC) biosensor for amperometric gas sensing. Sensors and Actuators B: Chemical, 2008. 135(1): p. 89-95.

178. Ibáñez-García, N., et al., Biparametric Potentiometric Analytical Microsystem Based on the Green Tape Technology. Electroanalysis, 2010. 22(20): p. 23762382.

179. Olivé-Monllau, R., et al., Integration of a sensitive carbon nanotube composite electrode in a ceramic microanalyzer for the amperometric determination of free chlorine. Sensors and Actuators B: Chemical, 2011. 151(2): p. 416-422.

180. Alves-Segundo, R., et al., Towards a monolithically integrated microsystem based on the green tape ceramics technology for spectrophotometric measurements. Determination of chromium (VI) in water. Microchimica Acta, 2010. 172(1-2): p. 225-232.

181. Gongora-Rubio, M.R., et al., LTCC manifold for heavy metal detection system in biomedical and environmental fluids. Sensors and Actuators B: Chemical, 2004. 103(1-2): p. 468-473.

182. Almeida, S.A.A., et al., Novel LTCC-potentiometric microfluidic device for biparametric analysis of organic compounds carrying plastic antibodies as ionophores: Application to sulfamethoxazole and trimethoprim. Biosensors and Bioelectronics, 2011. 30(1): p. 197-203. 


\section{CHAPTER III}

\section{Electrophoretically Deposited Polyanaline-Ag/AgO Hybrid Nanocomposite Based Electrochemical Immunosensor for Mediator Free Detection of Cortisol}

This chapter presents the development of a nanocomposite based immunosensing platform for the label-free and mediator-free electrochemical detection of cortisol. Coreshell $\mathrm{Ag} / \mathrm{AgO}$ nanoparticles and Polyanaline (PANI) is used to synthesize a hybrid nanocomposite film through electrophoretic deposition (EPD) onto Au substrate. For the first time, electrophoretically deposited $\mathrm{Ag} @ \mathrm{AgO}-\mathrm{PANI}$ hybrid nanocomposite is utilized for the immobilization of monoclonal Anti- $\mathrm{C}_{\mathrm{ab}}$ to realize a label-free and mediator-free electrochemical immunosensing platform for cortisol detection.

\subsection{Ag@AgO-PANI Hybrid Nanocomposite}

Multiple charged sites, large surface-to-volume ratio, high surface reaction activity, high catalytic efficiency and strong adsorption ability of organic-inorganic hybrid nanocomposites has prompted their use of as an immobilizing matrix to fabricate biosensors to enhance the signal in electrochemical biosensors [1]. Synergetic effect between parent molecules causes high electro-activity, stability and functionality. These properties make organic-inorganic hybrid nanocomposite as materials of choice for various applications such as solar cells, optoelectronic, energy storage, sensors, pharmaceuticals, drug delivery, biomedical devices etc [2].

Nanostructured PANI and its nanocomposite with metal (Au[1], carbon nanotubes[3], graphene[4] etc.,) have already shown potential as advanced functional materials for 
sensing technology [1]. The sensing performance of PANI has been improved via incorporating metal /metal oxide nanoparticles in its backbone. Au nanoparticle has been incorporated in PANI backbone to improve redox-stability, electro-activity, charge transfer kinetics, functionality, and stability of PANI. Dey et al described Au NP- PANI nanowires composite for immunosensor fabrication to detect prostate-specific-antigen, a biomarker used for the screening of prostate cancer[1]. Ag based nanomaterials has also shown its potential in sensing technology especially enhancing performance of biosensors due to its improved electro-activity and high electron transfer kinetics [5-7]. The high isoelectric point (IEP) of $\mathrm{AgO} \sim 10.4[8]$ has lead to high electrostatic interactions with biomolecules (IEP 4.2) due to the large differences in surface charges. PANI-Ag nanocomposites have also been explored, however till date researchers have only focused on its synthesis and characterization to explain improved optical and electrical properties $[9,10]$, pseudo-capacitive properties $[11,12]$ and electrochemical oxidation [13]. The nanocomposites of PANI-Ag have been used to detect vapours, wherein Ag enhances the charge transfer between polymers and nanoparticles[14]. However, potential application of these nanocomposites in biosensor development is not well explored. Hence there is scope to investigate the use of PANI-Ag nanocomposites as the immobilizing matrix in immunosensors.

\subsection{Materials and Methods}

Aniline, 1-ethyl-3-(3-dimethylaminopropyl)carbodiimide) (EDC), NHydroxysuccinimide (NHS) and cortisol were purchased from Sigma Aldrich. The monoclonal cortisol antibody $\left(\right.$ Anti- $\left.\mathrm{C}_{\mathrm{ab}}\right)$ 2330-4809 was procured from Abd serotec. All 
other chemicals were of analytical grade and were used without further purification. The working solutions of cortisol were prepared by dilution in PBS $(10 \mathrm{mM}, \mathrm{pH} 7.0,0.9 \%$ $\mathrm{NaCl})$.

The emeraldine base form of PANI was synthesized using oxidative polymerization of aniline in acidic media using ammonium persulphate (APS) as the oxidant [18]. Ag@AgO NP utilized in this research was obtained from QuantumSphere Inc. The nanoparticles were prepared through vapor phase condensation process by vaporizing $\mathrm{Ag}$ beyond its boiling point, until a sufficient rate of vaporization via resistance heat mechanism was achieved.

Thin films of Ag@AgO-PANI nanocomposite were electrophoretically deposited onto $\mathrm{Au}$ substrate employing colloidal suspension of PANI and $\mathrm{Ag} @ \mathrm{AgO}$ NP in acetonitrile. Deposition was carried out in a two-electrode cell configuration (Fig 3.1), with gold foil $(10 \mathrm{~mm} \times 30 \mathrm{~mm})$ as the counter electrode (Anode) and the pre-cleaned $\mathrm{Au}$ substrate as the working electrode (Cathode). $100 \mu 1$ of $\mathrm{Ag} @ \mathrm{AgO} \mathrm{NP}\left(0.1 \mathrm{mg} \mathrm{mL}^{-1}\right.$ in water) and $200 \mu \mathrm{L}$ of PANI $\left(0.02 \mu \mathrm{g} \mathrm{mL} \mathrm{L}^{-1}\right.$ in formic acid) were added to $2 \mathrm{ml}$ of acetonitrile, and sonicated for 1 minute to prepare the colloidal deposition solution. The film deposition parameter such as time and voltage were optimized and $60 \mathrm{~V}$ for $60 \mathrm{~s}$ was found suitable to fabricate electrochemically stable film with highest magnitude of response current. EPD deposited films were washed with deionized water to remove unbound particles and dried at room temperature in air. A standard process, reported previously [19-21], has been utilized to immobilize Anti- $\mathrm{C}_{\mathrm{ab}}$ covalently onto $\mathrm{Ag} @ \mathrm{AgO}-$ PANI /Au electrode. Anti- $\mathrm{C}_{\mathrm{ab}}$ was covalently bound on nanocomposite via EDC/NHS chemistry between amine group of PANI and $\mathrm{COOH}$ group of Anti- $\mathrm{C}_{\mathrm{ab}}$. The $\mathrm{Ag} @ \mathrm{AgO}-$ 
PANI nanocomposite film was fabricated via self-assembly of surface charged $\mathrm{Ag} @ \mathrm{AgO}$ NP in electro-active PANI backbone via electrostatic interaction. PANI chains provide a large surface area leading to a high electro-active surface area, surface to volume ration, and electro-activity. The multiple charge cites of $\mathrm{Ag} @ \mathrm{AgO} \mathrm{NP}$ interact with amine group of PANI to prevent the aggregation of PANI during electrophoretic deposition and form an inner-chain linkage. The nanoporous composite is beneficial for bioelectrode fabrication due to functionality, reduced diffusion resistance, and high surface charge. The high surface charge on $\mathrm{Ag} @ \mathrm{AgO}$ (IEP) 10.4) also support the Anti- $\mathrm{C}_{\mathrm{ab}}$ immobilization via strong electrostatic interaction.

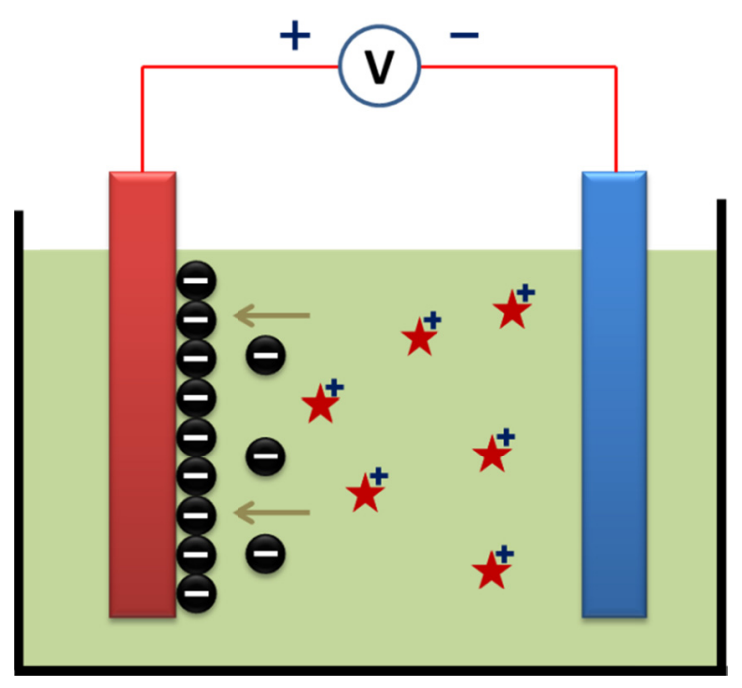

Fig 3.1 Schematic illustration of the two-electrode electrochemical cell for electrophoretic deposition.

The size of $\mathrm{Ag} @ \mathrm{AgO} \mathrm{NP}$ has been investigated using transmission electron microscopy (FEI Tecnai ${ }^{\mathrm{TM}}$ TEM). The appearance of nanocomposite and immunosensor has been investigated using atomic force microscopy (AFM). Electrochemical characterization of the fabricated nanocomposites electrode, immunoelectrode, and 
electrochemical response studies as a function of cortisol concentration have been carried out using cyclic voltammetry (CV) from $-0.4 \mathrm{~V}$ to $0.4 \mathrm{~V}$ in Phosphate Buffer Saline (PBS) at pH 7.0 containing $0.9 \% \mathrm{NaCl}$. Electrochemical studies were carried out in a $5 \mathrm{ml}$ threeelectrode cell with $\mathrm{Ag} / \mathrm{AgCl}$ as reference electrode and $\mathrm{Au}$ as counter electrode using the Autolab Potentiostat/Galvanostat (Eco Chemie, Netherlands). Electrochemical Impedance Spectroscopy (EIS) studies were carried out in PBS solution (10 mM, pH 7.0, $0.9 \% \mathrm{NaCl})$ containing a mixture of $5 \mathrm{mM} \mathrm{Fe}(\mathrm{CN})_{6}{ }^{3-14-}$ as redox probe at equilibrium potential without external biasing in the frequency range of $0.1-10^{5} \mathrm{~Hz}$ with $10 \mathrm{mV}$ amplitude.

\subsection{Results and Discussion}

\subsubsection{Optical Characterization of Ag@AgO NP\&Ag@AgO-PANI Nanocomposite.}

The TEM image (Fig. 3.2A) presents the uniformly distributed Ag@AgO nanoparticles on the TEM grid. The estimated average particle size was $\sim 5 \mathrm{~nm}$. Due to the high surface charge and small particle size, the nanoparticles appear to be slightly agglomerated. The enlarged TEM image core-shell nanostructure is shown in Fig 3.2B. The high resolution TEM study reveals that core of $\mathrm{Ag}(\sim 3 \mathrm{~nm})$ was encapsulated in a shell of $\mathrm{AgO}(\sim 2 \mathrm{~nm})$. The observed diffraction fringes indicate the crystalline structure of core-shell Ag@AgO nanoparticles. TEM based energy dispersive X-ray spectrum (EDX) of the nanoparticle

(Fig 3.2C) indicates that the primary constituents, $\mathrm{Ag}$ and $\mathrm{O}$ that form the core-shell structure of the $\mathrm{Ag} @ \mathrm{AgO}$ nanoparticle. 


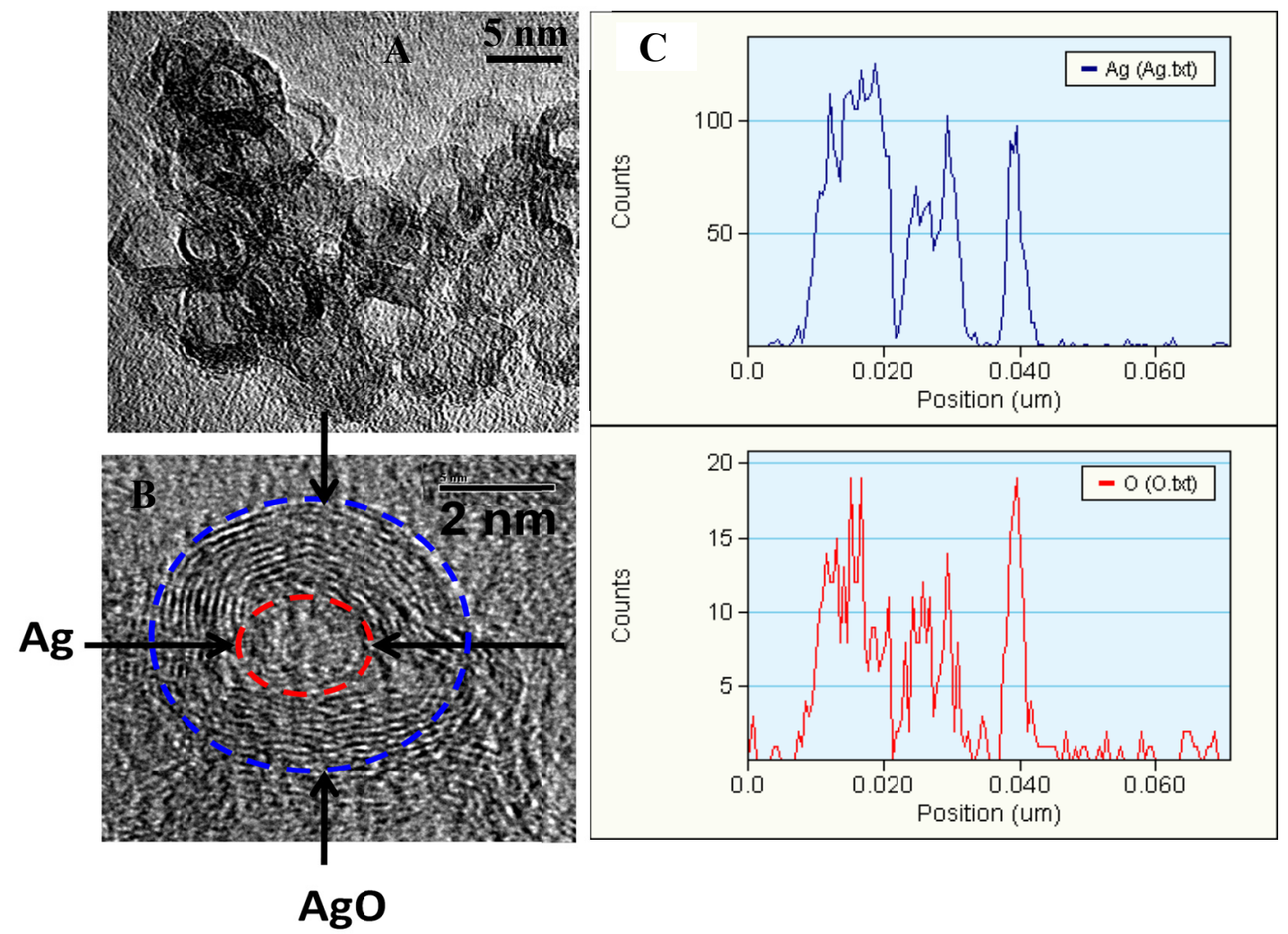

Fig 3.2 (a) TEM image of core-shell $\mathrm{Ag} @ \mathrm{AgO}$ nanoparticles high resolution, (b) image of $\mathrm{Ag} @ \mathrm{AgO}$ nanoparticles, and (c) EDX spectra of $\mathrm{Ag} @ \mathrm{AgO}$ nanoparticles.

The morphology of fabricated Ag@AgO-PANI nanocomposite was studied using 2D and 3D atomic force microscopy (AFM). The AFM image details the Ag@AgO nanoparticles (Fig 3.3a) uniformly distributed within PANI backbone. This morphology is attributed to hydrogen binding and weak Van der Waals forces resulting in the granular nonoporous appearance. The average height and mean roughness of nanocomposite has been estimated at $80 \mathrm{~nm}$ and $10 \mathrm{~nm}$ respectively. The elemental studies and formation of Ag@AgO-PANI nanocomposite was confirmed using EDX studies (Fig 3.3b), which indicates the presence of all related elements, confirming the fabrication of $\mathrm{Ag} @ \mathrm{AgO}-$ PANI nanocomposite. The morphological changes after the immobilization of Anti- $\mathrm{C}_{\mathrm{ab}}$ 
and Bovine Serum Albumin (BSA) have also been studied using AFM (Fig 3.3c). The granular nano-porous morphology of the nanocomposite film reformed to another wellarranged nano-porous morphology with an average height of $46 \mathrm{~nm}$ and mean roughness of $6.2 \mathrm{~nm}$ confirm the immobilization.
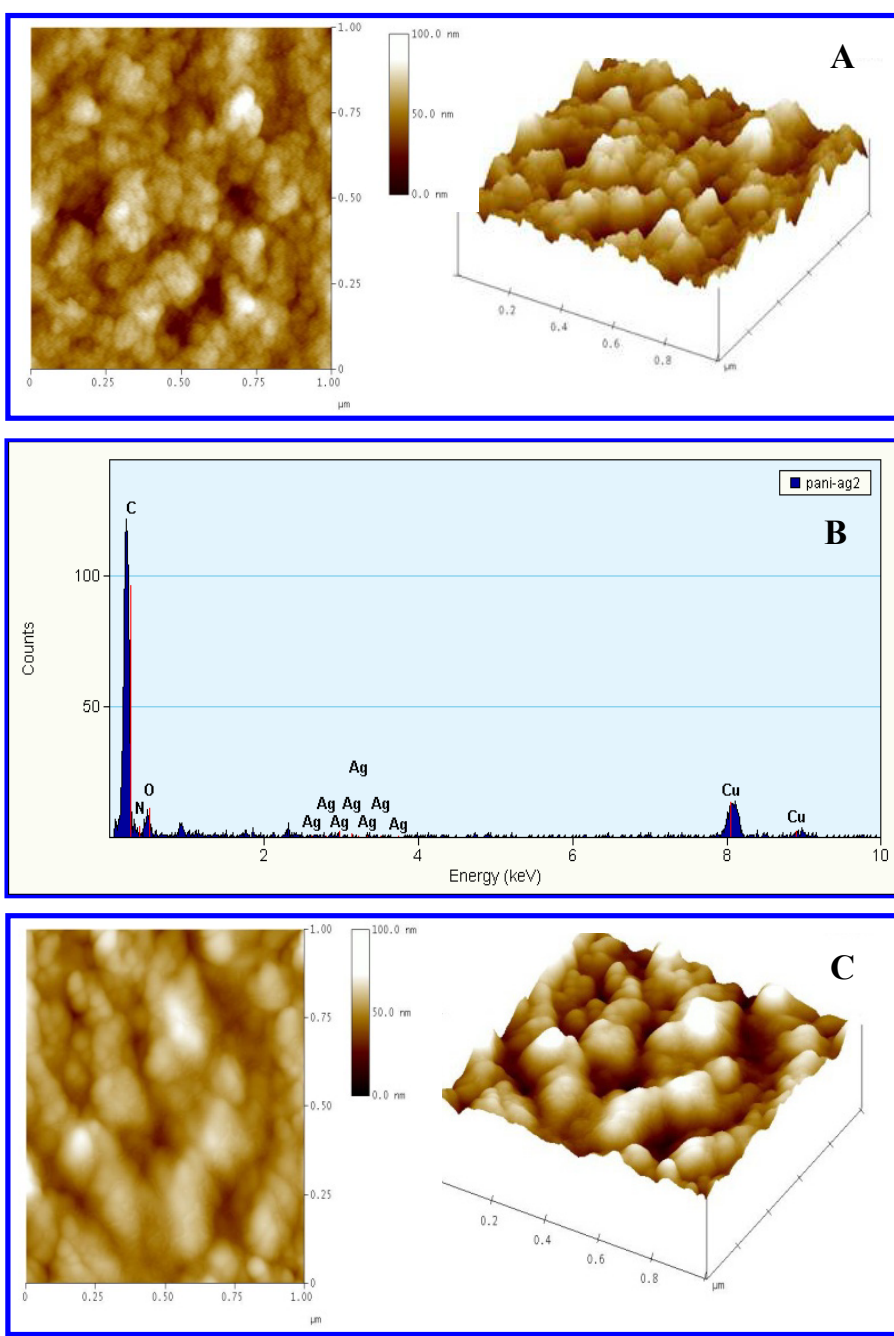

Fig 3.3 (a) AFM image of Ag@AgO-PANI/Au nanocompiste, (b) EDX spectra of $\mathrm{Ag} @ \mathrm{AgO}-\mathrm{PANI} / \mathrm{Au}$ nanocompiste, and (c) BSA/Anti-Cab $/ \mathrm{Ag} @ \mathrm{AgO}-\mathrm{PANI} / \mathrm{Au}$ immunoelectrode 


\subsubsection{Electrochemical Characterization}

The stepwise fabrication of the nanocomposite film and its functionalization for biosensing has been studied using electrochemical impedance spectroscopy (EIS). In EIS spectra (Nyquist plot)(Fig 3.4), the diameter of the semicircle estimates the charge transfer resistance $\left(\mathrm{R}_{\mathrm{CT}}\right)$ which reveals the electron transfer kinetics at the electrodeelectrolyte interface and is affected by the immuno reaction occurring at electrode interface. The Nyquist spectra of bare Au electrode exhibits a well-defined impedimetric behavior (curve a, $\mathrm{R}_{\mathrm{CT}}=120 \Omega$ ) i.e., semicircle in $5 \mathrm{mM} \mathrm{Fe}(\mathrm{II}) / \mathrm{Fe}(\mathrm{III})$ as redox moieties in PBS. The obtained data was fitted using an equivalent electrical circuit (inset, Fig 3.4). Increase in the diameter of semicircle for electrophoretically deposited PANI/Au electrode (curve $\mathrm{b}, \mathrm{R}_{\mathrm{CT}}=220 \Omega$ ) reveals that PANI film hinders the electron transport from the redox moieties to electrode indicating the fabrication of PANI film onto $\mathrm{Au}$ electrode. After the incorporation of $\mathrm{Ag} @ \mathrm{AgO}$ nanoparticles in PANI backbone, the diameter of the semicircle decreased (curve $\mathrm{c}, \mathrm{R}_{\mathrm{CT}}=175 \Omega$ ) revealing that the core shell nanoparticles enhanced the electron transport in the nanocomposite. This may be due to the uniformly distributed cationic AgO (IEP 10.4) in PANI backbone, which helps to increases the adsorption of redox moieties and to channelize the electron transport phenomena. The value of $\mathrm{R}_{\mathrm{CT}}$ was found to increase (curve $\mathrm{d}, 280 \Omega$ ) after the immobilization of Anti- $\mathrm{C}_{\mathrm{ab}}$ onto $\mathrm{Ag} @ \mathrm{AgO}-\mathrm{PANI} / \mathrm{Au}$ electrode. This suggests that the insulating nature of Anti- $\mathrm{C}_{\mathrm{ab}}$ hinders the electron transport from electrolyte to electrode indicative of successful immobilization of Anti- $\mathrm{C}_{\mathrm{ab}}$ onto nanocomposite surface via covalent binding with PANI and electrostatic interactions with $\mathrm{Ag} @ \mathrm{AgO}$. Further increment in $\mathrm{R}_{\mathrm{CT}}$ value (curve $\mathrm{d}, 400 \Omega$ ) after the immobilization of BSA is attributed to 
blocking of non-binding cites of Ant- $\mathrm{C}_{\mathrm{ab}} / \mathrm{Ag} @ \mathrm{AgO}-\mathrm{PANI} / \mathrm{Au}$ nanocomposite electrode, resulting in reduction of electron transport flow and binding on BSA protein molecules. EIS measurements were repeated in triplet and obtained results were found to be repeatable.

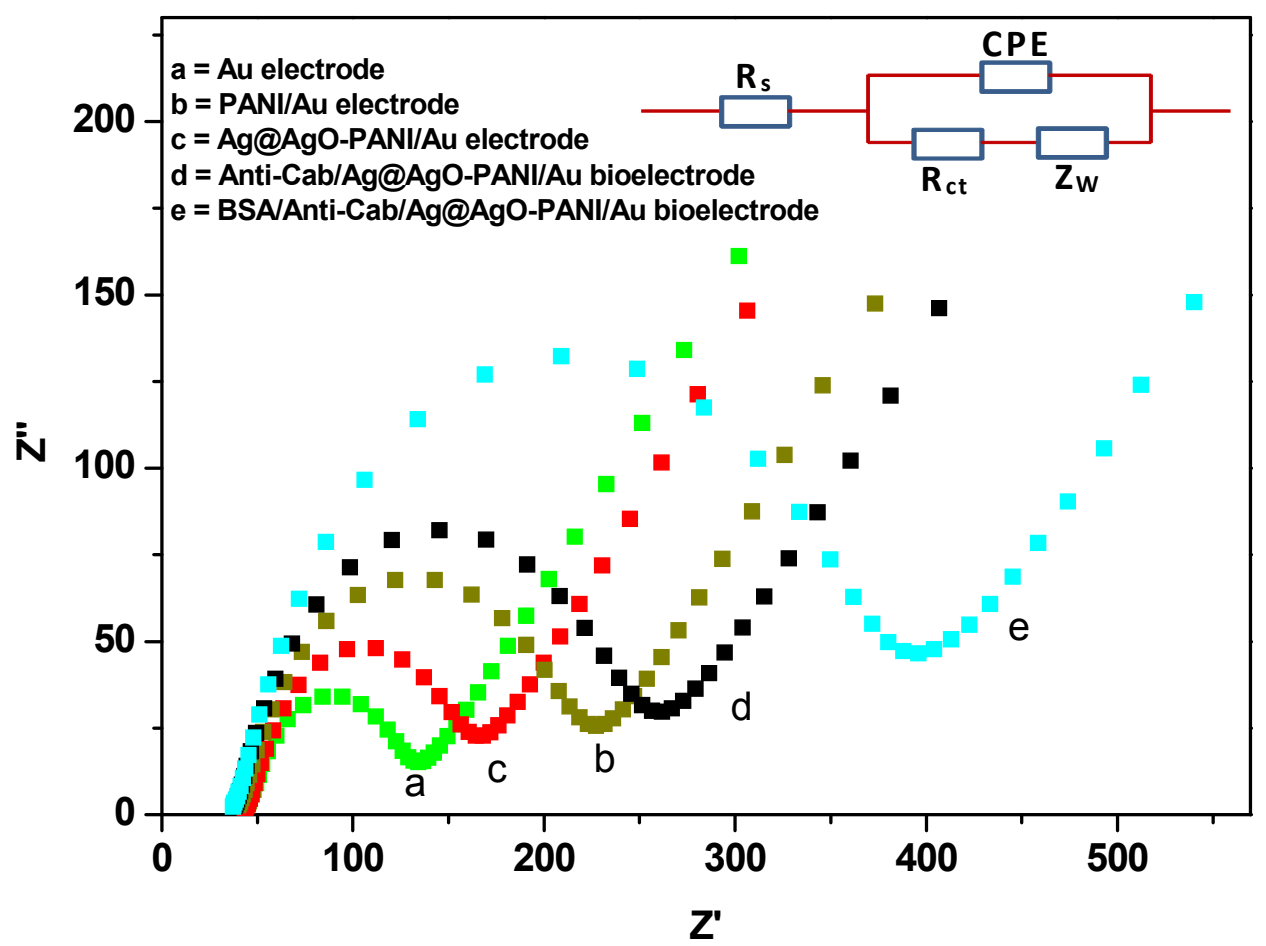

Fig 3.4 Characterization of stepwise fabrication of nanocomposite and immunosensor using EIS in PBS (10 nM, pH =7, 0.9\% NaCl) containing $5 \mathrm{mM} \mathrm{Fe(II)/Fe(III)} \mathrm{redox}$ moieties.

Cyclic voltammetry (CV, Fig 3.5) was also used to study the electro activity of the functionalized electrode to understand the electrochemical behavior of the electrode during the step-wise fabrication process. Bare Au electrode (inset a, Fig 3.5) exhibited oxidation and reduction current magnitude in the range of $\sim 10^{-9} \mathrm{~A}$ which is typical for bare $\mathrm{Au}$ electrode. Electrophoretically deposited PANI/Au electrode exhibited well 
defined oxidation $(0.1 \mathrm{~V})$ and reduction $(-0.1 \mathrm{~V})$ with increase in the magnitude of oxidation current response to $2 \times 10^{-4} \mathrm{~A}$ due to the oxidation of emaraldine base form of PANI. The magnitude of oxidation response current for Ag@AgO-PANI nanocomposite increases two fold to $4 \times 10^{-4} \mathrm{~A}$ in comparison to pure PANI. This suggests that incorporation of core-shell $\mathrm{Ag} @ \mathrm{AgO}$ nanopaprticles in PANI accelerates the electron transfer between electrons generated via oxidation of PANI to the electrode. The obtained well resolved sharp peak of Ag@AgO-PANI nanocomposite with amplified current value is due to the coordination between PANI and core-shell Ag@AgO nanoparticles. $\mathrm{Ag} @ \mathrm{AgO}$ nanoparticles also contribute in electron transport along with PANI through charge hopping via metallic conductors that result in enhanced charge transport via PANI. It is observed that doping or incorporation of electro-active metal/metal oxide ions in conductive polymeric backbone increases the effective surface and produces improved charge carriers per unit volume. In our system, the core-shell nanoparticles bind with the amine group of PANI (protonation) and the resulting nanocomposite become polarinic, wherein spin and charge are delocalized in the system. In Ag@AgO-PANI nanocomposites, the establishment of a polarons-bipolarons equilibrium results in enhanced mobility of charge carriers for the charge transport leading to increased charge density (curve c). In electrochemical reaction, the resistance to electron conduction is high in case of PANI and Au substrate; this may be due to some defects in PANI film. However, in nanocomposites, the presence of core-shell Ag@AgO nanoparticle in PANI backbone decreases the resistance and channelizes the electron transport revealing the enhanced charge density that result in fast electron transport via nanocomposite to substrate. 
The magnitude of the electrochemical current response decreases to $2.5 \times 10^{-4} \mathrm{~A}$ after the immobilization of Anti- $\mathrm{C}_{\mathrm{ab}}$ onto $\mathrm{Ag} @ \mathrm{AgO}-\mathrm{PANI} / \mathrm{Au}$ nanocomposite electrode indicating the binding of Anti- $\mathrm{C}_{\mathrm{ab}}$ (curve d). The decreased current response is due to hindrance in electron transport caused by the insulating antibodies. Furthermore, the magnitude of current response of BSA/Anti- $\mathrm{C}_{\mathrm{ab}} / \mathrm{Ag} @ \mathrm{AgO}-\mathrm{PANI} / \mathrm{Au}$ immunoelectrode is observed to be lower than that of Anti- $\mathrm{C}_{\mathrm{ab}} / \mathrm{Ag} @ \mathrm{AgO}-\mathrm{PANI} / \mathrm{Au}$ electrode (curve e). Decrease in current is attributed to the hindrance in charge transformation due to the insulating behavior of BSA via blocking of non-specific binding cites.

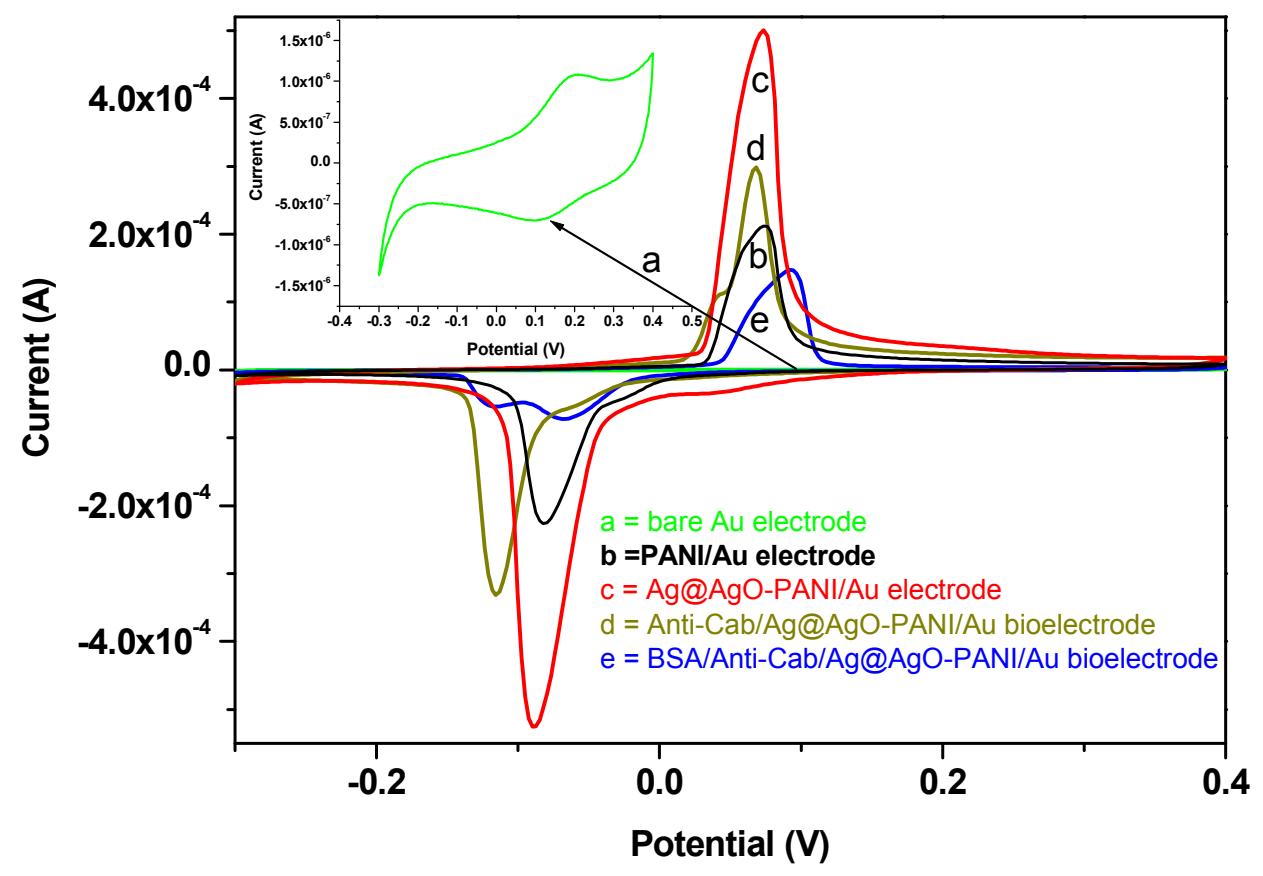

Fig 3.5 Characterization of stepwise fabrication of nanocomposite and immunosensor using $\mathrm{CV}$ in PBS (10 nM, $\mathrm{pH}=7,0.9 \% \mathrm{NaCl})$; inset: $\mathrm{CV}$ of bare Au electrode.

The sensing performance is dependent on electrochemical responsiveness of the immobilizing matrix and antibodies. In immunosensors, the net charge and activity of 
amino acids available on antibody is responsible for antigen-antibody binding. Also, during immobilization, the proteins may lead to rearrangement and conformational changes in native structure. These features of antibody are found to be electrolyte $\mathrm{pH}$ dependent and thus need to be optimized. It was observed that the magnitude of electrochemical response current of BSA/Anti-Cab $/ \mathrm{Ag} @ \mathrm{AgO}-\mathrm{PANI} / \mathrm{Au}$ immunoelectrode decreases on increasing $\mathrm{pH}$ from 6.0 to 8.0 (Fig 3.6; inset a). The magnitude of current response decreases gradually, and at $\mathrm{pH} 8$, immuno-electrode lost electrochemical activity due to highly basic medium. Similarly the magnitude of response current for Ag@AgO-PANI/Au electrode (Fig 3.6; inset b) decreases on increasing pH value due to de-protonation effect. Although current response values for Ag@AgO-PANI/Au and BSA/Anti- $\mathrm{C}_{\mathrm{ab}} / \mathrm{Ag} @ \mathrm{AgO}-\mathrm{PANI} / \mathrm{Au}$ were found to be better at $\mathrm{pH}$ values 6.0 and 6.5 , but the immunosensor response toward cortisol was found better at $\mathrm{pH}$ 7.0. Thus, $\mathrm{pH} 7.0$ was designated as the optimized working electrolyte $\mathrm{pH}$. Further, at $\mathrm{pH} 7.0$ electrolyte behaviors is adjacent to the neutral media, wherein, due to physiological condition electrolyte mimics biological condition. Moreover, the electrodes showed repeatable and reproducible electrochemical response behavior at $\mathrm{pH}$ 7.0. 


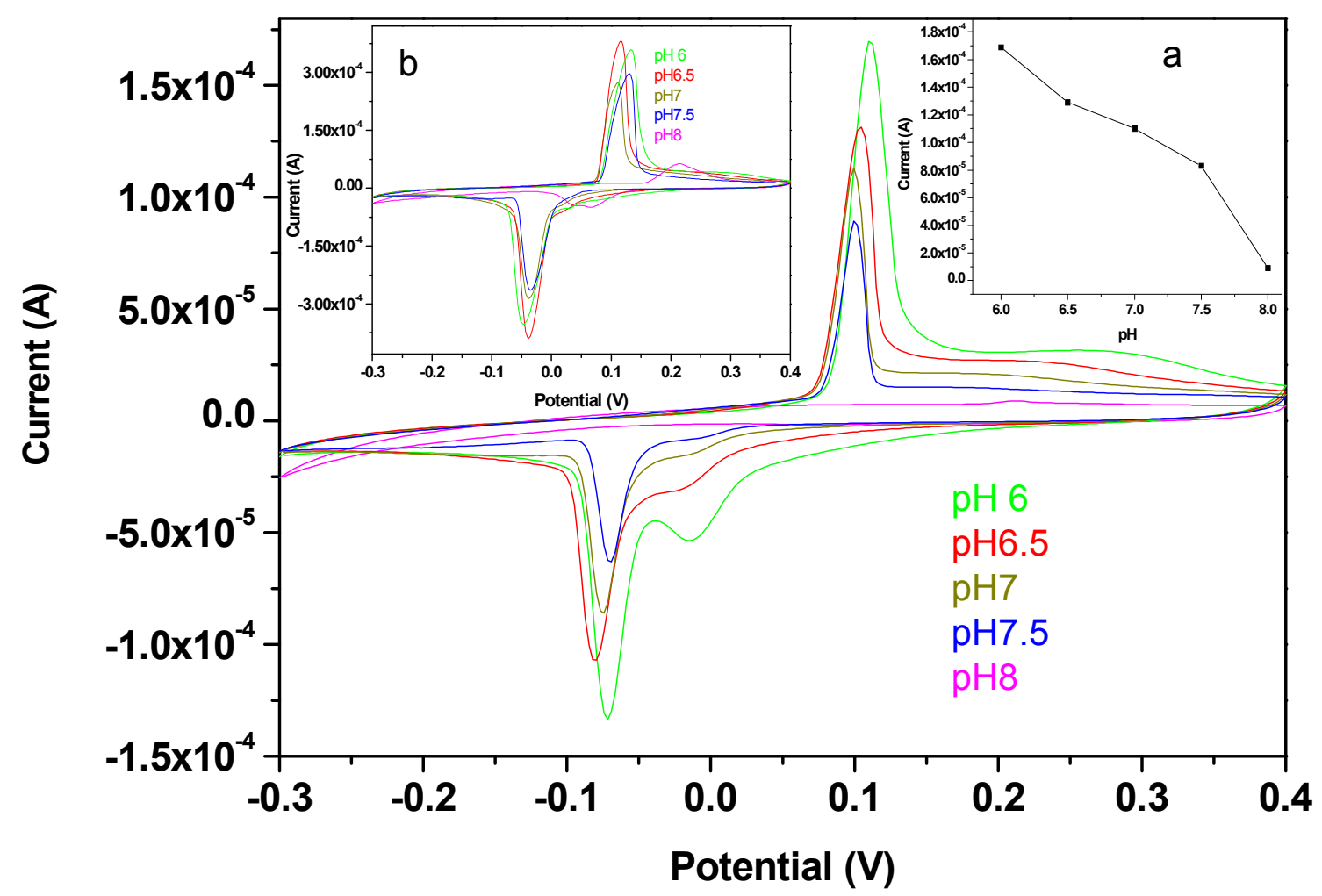

Fig 3.6 CV studies of immunoelectrode as a function of $\mathrm{pH}(6.0$ to 8.0$)$; inset: (a) magnitude of current v/s pH for BSA/Anti-C $\mathrm{ab}_{\mathrm{b}} / \mathrm{Ag} @ \mathrm{AgO}-\mathrm{PANI} / \mathrm{Au}$ immunoelectrode; (b) CV studies of Ag@AgO-PANI/Au nanocomposite electrode.

The effect of scan rate (10 to $100 \mathrm{mV} / \mathrm{s})$ on the electrochemical behavior of $\mathrm{Ag} @ \mathrm{AgO}-\mathrm{PANI} / \mathrm{Au}$ nanocomposite electrode and BSA/Anti-C $\mathrm{ab} / \mathrm{Ag} @ \mathrm{AgO}-\mathrm{PANI} / \mathrm{Au}$ immunoelectrode was studied and is presented in Fig 3.7 and Fig 3.8. The magnitude of electrochemical response current [cathodic $\left(I_{p}\right)$ and anodic $\left(I_{c}\right)$ ] for both electrodes are linearly dependent on the scan rate and follows equations 1-4. The observed well-defined stable redox peaks as a function of scan rate suggests that it is a surface-controlled electrochemical process and diffusion of electrons on surface is taking place in a controlled manner. The consistent peak-to-peak separation and stable peak 
position/current during repetition at constant scan rate was repeatable and indicate that nanocomposite electrode exhibits quasi-reversible process. The obtained sharp wellresolved peaks at higher scan rate without mediator suggest that Ag@AgO-PANI nanocomposite is electrochemically very active and is suitable for fabricating mediator free electrochemical biosensors. Moreover, the magnitude of both cathodic $\left(\mathrm{E}_{\mathrm{p}}\right)$ and anodic peak $\left(\mathrm{E}_{\mathrm{c}}\right)$ potentials for both nanocomposite electrode and immunoelectrode increases linearly as function of scan rate (equations 5-8) reveals the electron transport form redox moieties to the electrode is very feasible.

$\operatorname{Ip}(A)_{A g @ A g O-P A N I}=-9.01 \times 10^{-5} \mathrm{~A}+5.82 \times 10^{-5} \mathrm{~A}(\mathrm{~s} / \mathrm{mV}) \times$ Scan rate $(\mathrm{mV} / \mathrm{s})$ with $S D=$ $3.7 \times 10-6$ and $r^{2}=0.999$ 1

$I c(A)_{A g @ A g O-P A N I}=6.82 \times 10^{-5}-5.25 \times 10^{-5} \mathrm{~A}(\mathrm{~s} / \mathrm{mV}) \times$ Scan rate $(\mathrm{mV} / \mathrm{s})$ with $\mathrm{SD}=3.72 \times 10-6$

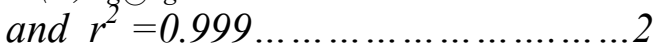

$I p(A)_{B S A / A n t i-C a b / A g @ A g O-P A N I}=5.97 \times 10^{-5}(\mathrm{~A})+1.47 \times 10^{-4} \mathrm{~A}(\mathrm{~s} / \mathrm{mV}) \times$ Scan rate $(\mathrm{mV} / \mathrm{s})$ with $S D=3.01 \times 10^{-4}$ and $r^{2}=0.999 \ldots \ldots \ldots . .3$

Ic $(A)_{B S A / A n t i-C a b / A g @ A g O-P A N I}=1.1 \times 10^{-4}(\mathrm{~A})-1.29 \times 10^{-4} \mathrm{~A}(\mathrm{~s} / \mathrm{mV}) \times$ Scan rate $(\mathrm{mV} / \mathrm{s})$ with $\mathrm{SD}$ $=2.28 \times 10^{-5}$ and $r^{2}=0.999 \ldots \ldots \ldots \ldots .4$

$E a(V)_{A g @ A g O-P A N I}=0.045 \mathrm{~V}+3.12 \times 10^{-4}(\mathrm{~s}) \times$ Scan rate $(\mathrm{mV} / \mathrm{s})$ with $S D=0.0026$, and $r^{2}=$

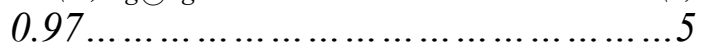

$E c(V)_{A g @ A g O-P A N I}=-0.048 V-3.6 \times 10^{-4}(\mathrm{~s}) \times$ Scan rate $(\mathrm{mV} / \mathrm{s})$ with $S D=0.0025$, and $r^{2}=$

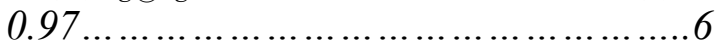

$E p(V)_{B S A / A n t i-C a b / A g @ A g O-P A N I}=0.07 \mathrm{~V}+6.2 \times 10^{-4}(\mathrm{~s}) \times$ Scan rate $(\mathrm{mV} / \mathrm{s})$ with $S D=0.47$, and $r^{2}=0.97 \ldots \ldots \ldots \ldots \ldots \ldots \ldots \ldots \ldots \ldots \ldots . .7$

$E c(V)_{B S A / A n t i-C a b / A g @ A g O-P A N I}=-0.05 V+0.0012(\mathrm{~s}) \times$ Scan rate $(\mathrm{mV} / \mathrm{s})$ with $S D=0.0017$, and $r^{2}=0.99 \ldots \ldots \ldots \ldots \ldots \ldots \ldots \ldots \ldots \ldots$

The surface concentrations of redox species on PANI /Au electrode, Ag@AgOPANI/Au electrode and BSA/Anti- $\mathrm{C}_{\mathrm{ab}} / \mathrm{Ag} @ \mathrm{AgO}-\mathrm{PANI} / \mathrm{Au}$ immunoelectrodes have been estimated using $i_{p}=0.227 n F A C_{o} * k^{o} \exp \left[-\alpha n_{a} F / R T x\left(E_{p}-E_{o}^{\prime}\right)\right]$, where $\mathrm{i}_{\mathrm{p}}$ is the anodic peak current, $\mathrm{n}$ is the number of electrons transferred (2), $\mathrm{F}$ is the Faraday constant ( $\left.96485.34 \mathrm{C} \mathrm{mol}^{-1}\right), \mathrm{A}$ is surface area $\left(0.25 \mathrm{~cm}^{2}\right), \mathrm{R}$ is the gas constant $\left(8.314 \mathrm{~J} \mathrm{~mol}^{-1} \mathrm{~K}^{-}\right.$ 
$\left.{ }^{1}\right), \mathrm{C}_{\mathrm{o}} *$ is surface concentration of the ionic species of electrode surface $\left(\mathrm{mol} \mathrm{cm} \mathrm{cm}^{-2}\right), E_{p}$ is the peak potential and $E_{o} !$ is the formal potential. $-\alpha n_{a} F / R T$ and $k^{o}$ (rate constant (0.5)), correspond to the slope and intercept of $\ln \left(i_{p}\right)$ verses $\mathrm{E}_{\mathrm{p}}-\mathrm{E}_{0} !$ curve at different scan $\operatorname{rates}[3]$.

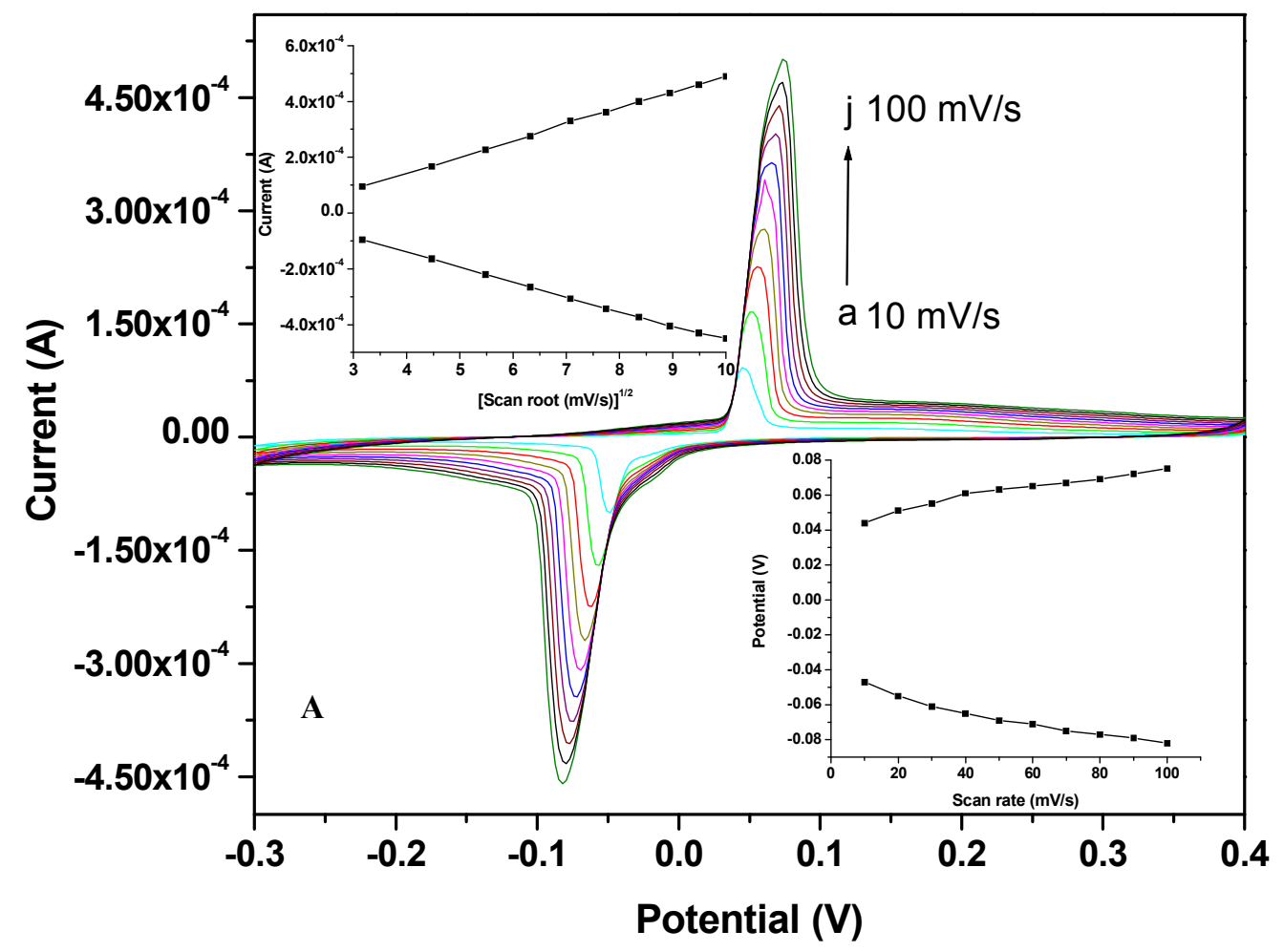

Fig 3.7 CV studies of $\mathrm{Ag} @ \mathrm{AgO}-\mathrm{PANI} / \mathrm{Au}$ electrode as a function of scan rate (10$100 \mathrm{mV} / \mathrm{s}$ ); inset: (a) magnitude of current response v/s square root of scan rate; (b) Redox potential $\mathrm{v} / \mathrm{s}$ scan rate. 


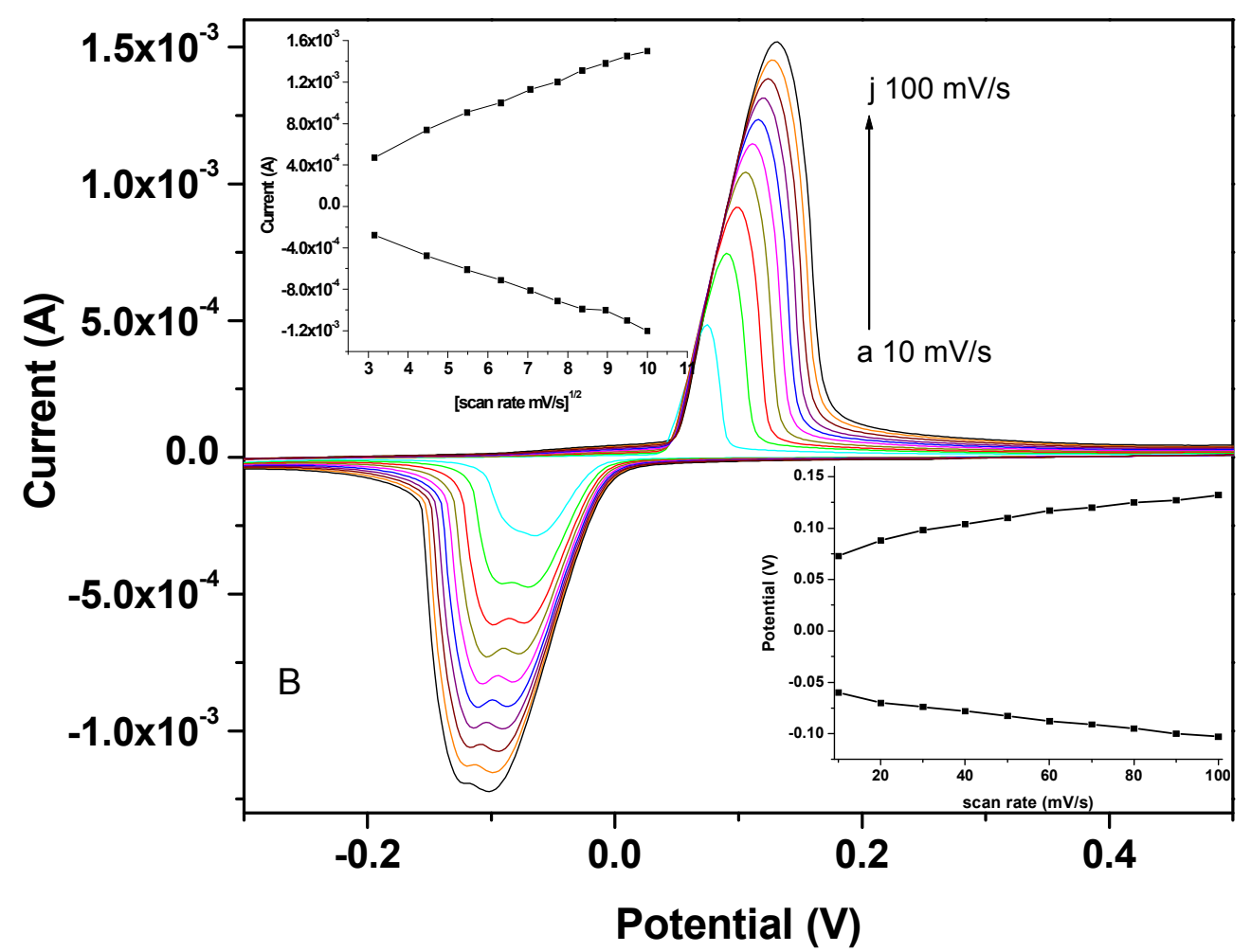

Fig 3.8 CV studies of BSA/Anti- $\mathrm{C}_{\mathrm{ab}} / \mathrm{Ag} @ \mathrm{AgO}-\mathrm{PANI} / \mathrm{Au}$ electrode as a function of scan rate $(10-100 \mathrm{mV} / \mathrm{s})$; inset; (a) magnitude of current response $\mathrm{v} / \mathrm{s}$ square root of scan rate; (b) redox potential v/s scan rate.

The surface concentration of redox species on $\mathrm{Ag} @ \mathrm{AgO}-\mathrm{PANI} / \mathrm{Au}$ electrode surface is found to be higher than PANI electrode revealing that the interaction of core-shell Ag@AgO nanoparticle with multi-charged aromatic structured PANI help to uncoil PANI to form open conformation that results in increased electro-active surface area. This suggests that larger numbers of redox species adsorb onto nanocomposite electrode and contribute to the higher faradic current due to open conformation. The value of cathodic response current is almost equal to the anodic response current value. The variation of just $\sim 0.05 \mu \mathrm{A}$ confirms the reversible stable electrochemical behavior of 
nanocomposite. This is due to the presence of core-shell $\mathrm{Ag} @ \mathrm{AgO}$ nanoparticles in PANI matrix as a dopant that stabilize electrochemistry and leads to the steady and reversible behavior of Ag@AgO-PANI nanocomposite. However, the value of surface concentration of redox moieties decreases for $\mathrm{BSA} / \mathrm{Anti}-\mathrm{C}_{\mathrm{ab}} / \mathrm{Ag} @ \mathrm{AgO}-\mathrm{PANI} / \mathrm{Au}$ immunoelectrode due to the blocking of the electro-active cites via BSA.

The heterogeneous electron transfer rate constant $(k \mathrm{~s})$ for BSA/Anti- $\mathrm{C}_{\mathrm{ab}} / \mathrm{Ag} @ \mathrm{AgO}-$ $\mathrm{PANI} / \mathrm{Au}$ immunoelectrode was estimated as $5.2 \mathrm{~s}^{-1}$ using the Laviron model as $k s$ $=m n F v / R T$, where $m$ is peak-to-peak separation, $\mathrm{F}$ is Faraday constant, $\mathrm{v}$ is scan rate (mV/s), $n$ is the number of transferred electrons and $R$ is gas constant[15]. The obtained $k$ s value $(T=298 \mathrm{~K}, n=2 m=0.103 \mathrm{~V}$ and $v=100 \mathrm{mV})$ is similar to other highly electroactive nanoparticles based bioelectrodes indicating fast electron transfer between immobilized biomolecules and electrode [15-17].

\subsubsection{Electrochemical response study for cortisol detection}

The electrochemical response current of $\mathrm{BSA} / \mathrm{Anti}-\mathrm{C}_{\mathrm{ab}} / \mathrm{Ag} @ \mathrm{AgO}-\mathrm{PANI} / \mathrm{Au}$ immunoelectrode has been studies using CV technique in triplet set using PBS $(\mathrm{pH} 7$ containing $0.9 \% \mathrm{NaCl}$ ) at scan rate $30 \mathrm{mV} / \mathrm{s}$ as a function of cortisol concentration ranging from $1 \mathrm{pM}$ to $1 \mu \mathrm{M}$. All immunoelectrodes were fabricated in identical condition and exhibit current value with a maximum variation of 3\%. Fig 3.9 depicts that the magnitude of electrochemical current response decreases as a function of increasing cortisol concentration at an incubation time of $30 \mathrm{~min}$. This is due to the formation of insulating immunocomplex formed between antibody and cortisol that hinders electron transport. A calibration curve between the magnitude of current response and logarithm 
of cortisol concentration has been plotted (inset, Fig 3.9), which reveals a linear correlation up to $1 \mu \mathrm{M}$ and follow the equation $I_{a}(A)=-3.53 \times 10^{-4} A M-66 \mu A x$ Log[cortisol conc. $(M)]$ with $S D=9.01 \times 10^{-6}$. The immunosensor exhibited a linear detection range from $1 \mathrm{pM}$ to $1 \mu \mathrm{M}$ at a sensitivity of $66 \mu \mathrm{A} \mathrm{M}^{-1}$ (higher than ELISA) with a regression coefficient of 0.998 . The detection limit of the fabricated BSA/Anti$\mathrm{C}_{\mathrm{ab}} / \mathrm{Ag} @ \mathrm{AgO}-\mathrm{PANI} / \mathrm{Au}$ immunosensor is estimated as $0.64 \mathrm{pM}$ using $3 \delta_{b} / m$, where $m$ is the slope of the calibration graph and $\delta_{b}$ is the standard deviation of the baseline signal. The obtained detection limit is lower than ELISA technique and other immunosensors reported in literature.

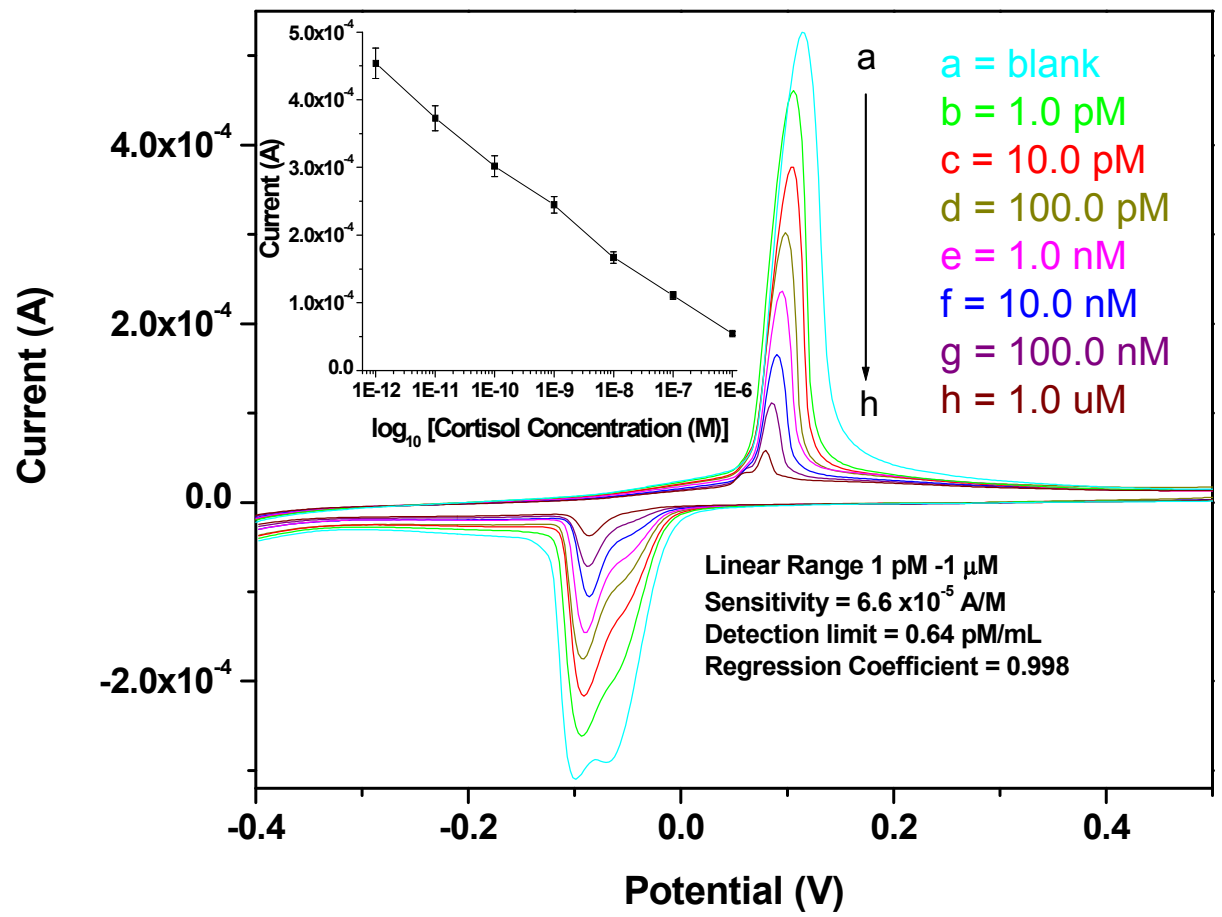

Fig 3.9 Electrochemical response studies of $\mathrm{BSA} / \mathrm{Anti}-\mathrm{C}_{\mathrm{ab}} / \mathrm{Ag} @ \mathrm{AgO}-\mathrm{PANI} / \mathrm{Au}$ immunoelectrode as function of cortisol concentration (1pM to $1 \mu \mathrm{M})$ using PBS (pH 6.5, $0.9 \% \mathrm{NaCl}$ ); inset: calibration curve between magnitude of response current and logarithm of cortisol concentration. 


\subsection{Conclusion}

The first research activity undertaken was to establish an immunosensing platform for the electrochemical detection of cortisol. A nanostructured immunosensing platform based on a hybrid nanocomposite film of core-shell $\mathrm{Ag} @ \mathrm{AgO}$ nanoparticles and Polyanaline (PANI) has been synthesized using electrophoretic deposition (EPD) onto Au substrate. The core-shell $\mathrm{Ag} @ \mathrm{AgO}$ nanoparticle $(\sim 5 \mathrm{~nm})$ has successfully been embedded in PANI backbone to make a highly electro-active Ag@AgO-PANI nanocomposite. For the first time, the electrophoretically deposited nanomaterial was utilized for the immobilization of monoclonal Anti- $\mathrm{C}_{\mathrm{ab}}$ to fabricate a label-free and mediator free electrochemical immunosensor for cortisol detection. Results of electrochemical characterization suggest that $\mathrm{Ag} @ \mathrm{AgO}$ nanoparticle act as doping agents for PANI and result in the increase charge density and stabilize electrochemical behavior, resulting in enhanced charge transfer from redox moieties to electrode. This increased electro-active platform with nano-porous morphology and functionality provides a friendly environment to immobilize Anti- $\mathrm{C}_{\mathrm{ab}}$. The immunosensor was tested as a function of cortisol concentrations in phosphate buffer saline (PBS) using cyclic voltammetry and found to exhibit a detection limit of $0.64 \mathrm{pg} \mathrm{mL}^{-1}$ (estimated using $3 \sigma$ formula), a linear range from $1 \mathrm{pM}$ to $1 \mu \mathrm{M}$, with a sensitivity of $66 \mu \mathrm{A} / \mathrm{M}$.

The ultimate goal of this research is to develop a point-of-care immunosensing platform for the detection of cortisol. The next logical step was to integrate the immunosensing platform onto a micromachined co-planar electrode set-up. The developed Ag@AgO-PANI based immunosensor was unable to be successfully integrated onto microfabricated electrodes due to a high degree of non-specific 
electrophoretic deposition of the hybrid nanocomposite onto the base substrate (Glass) leading to electrical shorting of the fabricated electrodes. To overcome challenges related to system integration, an alternate strategy, involving the use of self-assembled monolayer as the nanomaterial was explored and is presented in the next chapter. 


\subsection{References}

1. Dey, A., et al., Mediator free highly sensitive polyaniline-gold hybrid nanocomposite based immunosensor for prostate-specific antigen (PSA) detection. Journal of Materials Chemistry, 2012. 22(29): p. 14763.

2. Sanchez, C., et al., Applications of hybrid organic-inorganic nanocomposites. Journal of Materials Chemistry, 2005. 15(35-36): p. 3559-3592.

3. Dhand, C., et al., Polyaniline-carbon nanotube composite film for cholesterol biosensor. Analytical Biochemistry, 2008. 383(2): p. 194-199.

4. $\quad$ Feng, X.-M., et al., One-Step Electrochemical Synthesis of Graphene/Polyaniline Composite Film and Its Applications. Advanced Functional Materials, 2011. 21(15): p. 2989-2996.

5. Luo, X., et al., Application of Nanoparticles in Electrochemical Sensors and Biosensors. Electroanalysis, 2006. 18(4): p. 319-326.

6. Cao, Y., et al., Sensing purine nucleoside phosphorylase activity by using silver nanoparticles. Biosensors and Bioelectronics, 2010. 25(5): p. 1032-1036.

7. Ren, X., et al., Using silver nanoparticle to enhance current response of biosensor. Biosensors and Bioelectronics, 2005. 21(3): p. 433-437.

8. Peterson, K.A., et al., Novel Microsystem Applications with New Techniques in Low-Temperature Co-Fired Ceramics. International Journal of Applied Ceramic Technology, 2005. 2(5): p. 345-363.

9. $\quad$ Correa, C.M., et al., One-pot synthesis of a polyaniline-silver nanocomposite prepared in ionic liquid. RSC Advances, 2012. 2(7): p. 3088.

10. Gupta, K., P.C. Jana, and A.K. Meikap, Optical and electrical transport properties of polyaniline-silver nanocomposite. Synthetic Metals, 2010. 160(1314): p. 1566-1573.

11. Sawangphruk, M. and T. Kaewsongpol, Direct electrodeposition and superior pseudocapacitive property of ultrahigh porous silver-incorporated polyaniline films. Materials Letters, 2012. 87: p. 142-145.

12. Patil, D.S., et al., Investigations on silver/polyaniline electrodes for electrochemical supercapacitors. Physical Chemistry Chemical Physics, 2012. 14(34): p. 11886

13. Paulraj, P., et al., Single pot synthesis of polyaniline protected silver nanoparticles 
by interfacial polymerization and study its application on electrochemical oxidation of hydrazine. Colloids and Surfaces A: Physicochemical and Engineering Aspects, 2011. 377(1-3): p. 28-34.

14. Li, Z.-F., et al., Amplified response and enhanced selectivity of metal-PANI fiber composite based vapor sensors. Sensors and Actuators B: Chemical, 2012. 161(1): p. 390-395.

15. Kaushik, A., et al., Iron oxide-chitosan nanobiocomposite for urea sensor. Sensors and Actuators B: Chemical, 2009. 138(2): p. 572-580.

16. Zhang, L., et al., Attachment of gold nanoparticles to glassy carbon electrode and its application for the direct electrochemistry and electrocatalytic behavior of hemoglobin. Biosensors and Bioelectronics, 2005. 21(2): p. 337-345.

17. Zhao, Y.-D., et al., The interface behavior of hemoglobin at carbon nanotube and the detection for H2O2. Talanta, 2005. 65(2): p. 489-494.

18. Kaushik, A., et al., Fabrication and Characterization of PolyanilineZnO Hybrid Nanocomposite Thin Films. Journal of Nanoscience and Nanotechnology, 2008. 8(4): p. 1757-1761.

19. Arya, S.K., et al., Antibody functionalized interdigitated [small mu ]-electrode (ID[small mu ]E) based impedimetric cortisol biosensor. Analyst, 2010. 135(8): p. 1941-1946.

20. Arya, S.K., et al., Dithiobis(succinimidyl propionate) modified gold microarray electrode based electrochemical immunosensor for ultrasensitive detection of cortisol. Biosensors and Bioelectronics, 2010. 25(10): p. 2296-2301.

21. Arya, S.K., et al., Antibody modified gold micro array electrode based electrochemical immunosensor for ultrasensitive detection of cortisol in saliva and ISF. Procedia Engineering, 2010. 5: p. 804-807. 


\section{CHAPTER IV}

\section{Dithiobis (succinimidylpropionte) (DTSP) Modified Interdigitated Microelectrodes ( $\mu$ IDE) for Electrochemical Immunosensing of Cortisol}

\subsection{Self-Assembled Monolayers (SAM)}

Self-assembled monolayers (SAMs) have recently come into focus due to their potential applications to biosensors and bio-molecular electronics[1]. Self-assembled monolayer is a layer of molecular thickness formed by self-organization of molecules in an ordered manner by chemisorption on a solid surface[2]. SAM consists of three identifiable parts: a surface-active head group that binds strongly to a substrate, an alkyl chain giving stability to the assembly by van der Waals interactions, and a functional group that plays an important role in terms of coupling of a biomolecule to a monolayer. Through proper selection of terminal functional group, specific surface/solution interactions (covalent, electrostatic or hydrophobic) can be exploited to immobilize molecules at an interface. SAM functionalized with large, delicate ligands for biological studies can be prepared either by molecules with an attached ligand or more commonly attaching ligands to the surface of a preformed SAM[3].

The stability, uniform surface structure and relative ease of varying thickness of a SAM make it suitable for development of biosensors. And the immobilization of biomolecules on a SAM requires very small amount and desired analytes can be easily detected via various transduction modes. The use of an appropriate SAM helps in oriented and controlled immobilization of biomolecules [4]. SAM modification can result 
in development of surfaces with large/complex ligands and molecules needed for biology and biochemistry[5]. Also modification of exposed surface of a SAM offers many advantages such as simplicity to prepare functionalized surfaces, incorporation of synthetically non-compatible ligands into SAMs, generation of samples with multifunctional groups sensitive to different chemistries, etc.[6]. A new strategy has been demonstrated for specific and efficient immobilization of protein using semi-fluorinated SAM. The study reveals that such SAMs reduce non-specific protein adsorption and result in highly sensitive measurements with low limits of detection. The advantages of SAMs lie in their integration with a biological recognition element for development of biosensors [7]. For fabrication of a biosensor, immobilization of a biomolecule on a SAM surface is an important concern. SAMs for biosensing applications can be modified by adopting many other strategies. An effective combination of antibody-antigen specificity with transducer in a biosensor device could provide basis for direct detection of wide range of analytes with specificity and high sensitivity. Thus SAM-based immunosensors have implications towards development of a highly efficient protein sensor chip. In this research activity, SAM of Dithiobis(succinimidylpropionte) (DTSP) has been utilized to functionalize microfabricated $\mathrm{Au}$ electrodes for the immobilization of Anti-Cortisol antibodies for label-free, electrochemical detection of Cortisol.

\subsection{Dithiobis (succinimidylpropionte) (DTSP)}

DTSP, also referred to in literature as Lomant's reagent is a small molecular weight (404.42 grams/mole) molecule given by the chemical formula $\mathrm{C}_{14} \mathrm{H}_{16} \mathrm{~N}_{2} \mathrm{O}_{8} \mathrm{~S}_{2}$. Fig 4.1 presents a schematic illustration of the molecular assembly of DTSP. DTSP has a disulfide bond that is readily cleavable using a reducing agent such as sodium 
borohydride $\left(\mathrm{NaBH}_{4}\right)$. The thiol structure of DTSP provides strong chemisorption on metallic surfaces such as $\mathrm{Au}$ and an amine-reactive ester reacts rapidly with any primary amine-containing molecule such as antibodies to form an amide bond[8]. DTSP shows stable functioning in the $\mathrm{pH}$ range $7-9$ which is the ideal $\mathrm{pH}$ range for protein activities, thereby making it an ideal choice for incorporation in immunosensors. Also, DTSP retains its chemical stability after binding with a protein such as antibodies[9]. The highly ordered structure of DTSP and packing density can be temporally controlled to form a monolayer of desired porosity and order.

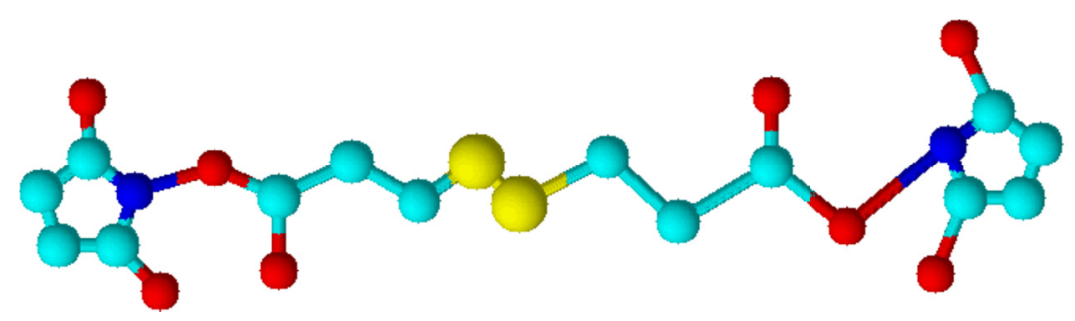

Fig 4.1 Three-dimensional molecular assembly of DTSP

\subsection{Interdigitated Microelectrode ( $\mu$ IDE) Design}

A conventional electrochemical measurement set-up consists of the working electrode, counter electrode and a reference electrode immersed in an electrolyte solution (as seen in Chapter III). The working electrode is where the adsorption of the target analyte is monitored. While this set-up is ideal for demonstration of sensing principles, it is severely restricted to a laboratory technique and is not feasible for use in integrated systems that are needed for point of care applications.

The advent and maturation of microfabrication techniques has allowed the fabrication of microstructures of sensing electrodes in order to incorporate the three electrode system in a co-planar set-up and improve the electrochemical response through 
smart design of the electrode shapes and dimensions. Microfabricated planar electrodes with well-defined electrode patterns provide the platform that is required for biosensing, as well as convenient integration with fluidic automation set-up. Over the years, many electrode designs have been successfully fabricated and tested for biosensing applications [10-12]. Of these, the interdigitated electrode design ( $\mu$ IDE) offers many advantages. The combination of IDE's along with electrochemical detection techniques such as Cyclic Voltammetry and Electrochemical Impedance Spectroscopy provides the necessary sensitivity for the detection of low concentrations of the analyte [13-15]. The efficiency of current collection resulting from the redox cycling increases significantly as the width and the distance between the interdigitated electrodes decrease (Fig 4.2a).

In this dissertation, interdigitated microelectrodes have been designed and fabricated for application in immunosensing of cortisol using electrochemical detection techniques (Fig 4.2b). The spacing and width of the electrode fingers is designed for $5 \mu \mathrm{m}$. This number is chosen to obtain repeatable fabrication and to maintain consistency between the many electrode chips that are batch fabricated. The total surface area of the interdigitated part of the working and counter electrode is $6.39 \mathrm{~mm}^{2}$. The thickness of the electrode is designed to be $50 \mathrm{~nm}$ of chrome and $150 \mathrm{~nm}$ of gold 

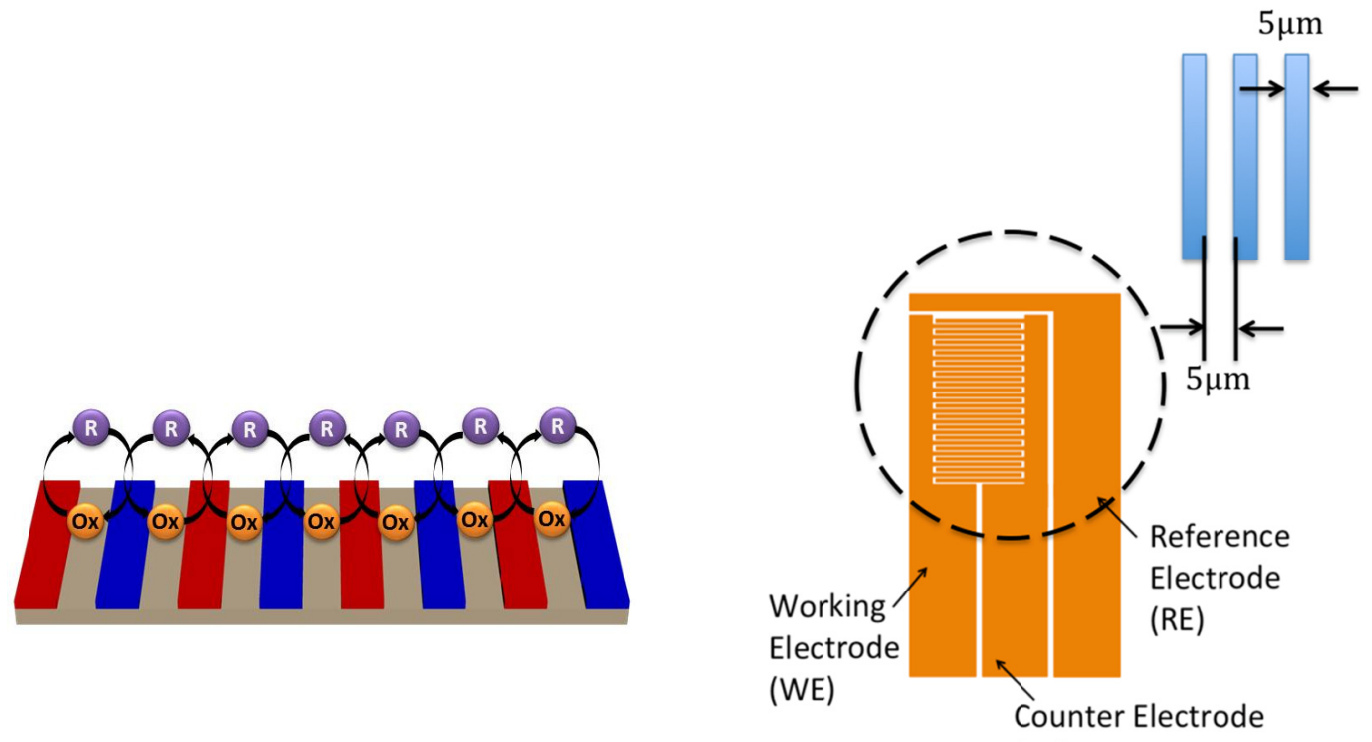

(CE)

Fig 4.2 (a) Schematic illustration of improved redox cycling in interdigitated microelectrodes; (b) Interdigitated Microelectrode design

\section{4 $\mu$ IDE Fabrication Process}

Fig 4.3 presents the schematic illustration of the microfabrication process flow for the fabrication of the interdigitated microelectrodes. The interdigitated electrodes were fabricated on a 4" glass wafer to reduce leakage currents that are typically observed in silicon substrate (Fig 4.3a). Prior to usage, the glass wafer was cleaned by rinsing in DI water, Isopropyl Alcohol, Acetone, Methanol and DI water in the order mentioned. The cleaned wafer was blow dried and a dehydration bake was carried out on in an oven at $100^{\circ} \mathrm{C}$ for 10 minutes to remove any residual water molecules. A thin layer of negative photoresist (PY-3000) was spin coated (Laurell Technologies) onto the glass wafer at $3000 \mathrm{rpm}$ for 40 seconds to obtain a film thickness of $6 \mu \mathrm{m}$ (Fig 4.3b). The photoresist film was soft baked at $150^{\circ} \mathrm{C}$ for 60 seconds. The electrode design was transferred onto the photoresist using contact photolithography (EVG Group). The exposed photoresist 
was post-exposure baked at $100^{\circ} \mathrm{C}$ for 60 seconds followed by developing in the appropriate developing solution (Fig 4.3c) to realize the features. Next, the metallization process was carried out by deposition of $\mathrm{Cr}(20 \mathrm{~nm})$ as the adhesion layer, followed by $\mathrm{Au}$ (150nm) using an E-beam evaporator (Fig 4.3d). The sacrificial photoresist was then removed by a lift-off process by immersing the wafer in acetone to realize the microelectrode pattern (Fig 4.3e). A passivation layer is then deposited by spin-coating SU-8 onto the fabricated electrode and using a $2^{\text {nd }}$ photolithography process, openings are patterned to only expose the functional part of the electrodes and the probing pads for connection (Fig 4.3f). The fabricated wafer is then diced to obtain the individual electrode chips. Fig 4.4 presents a microscopic image of the microfabricated interdigitated electrode and Fig 4.4b presents the scanning electron micrographs (SEM) of the interdigitated electrodes.

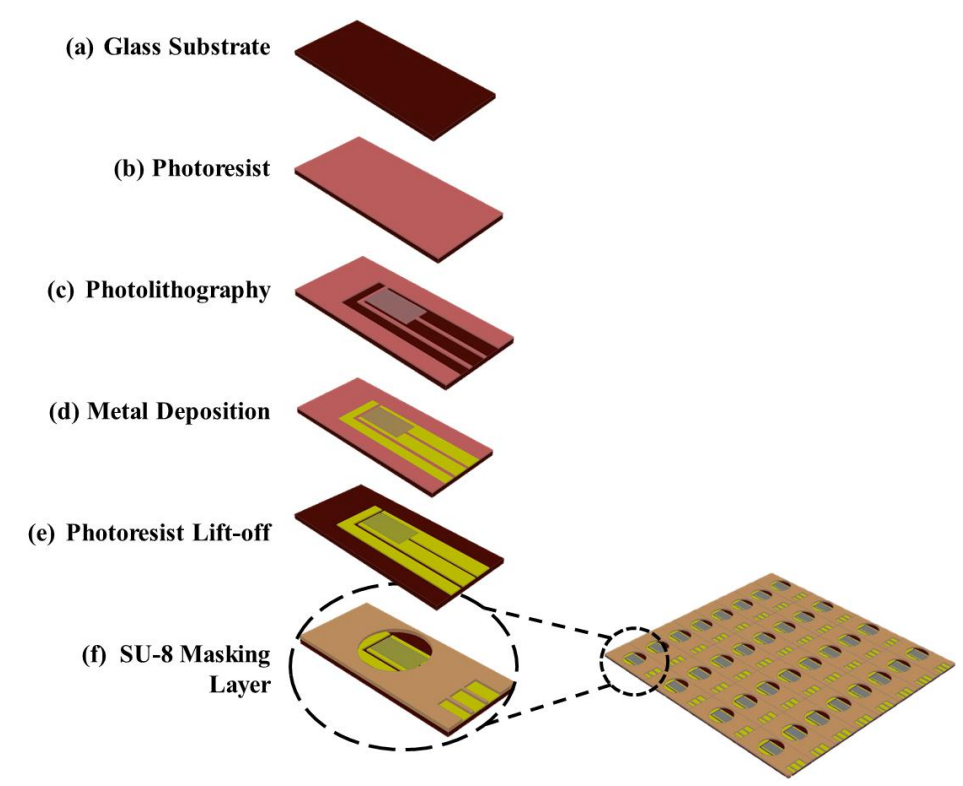

Fig 4.3 Schematic illustration of the microfabrication process flow for fabrication of the interdigitated microelectrodes. 


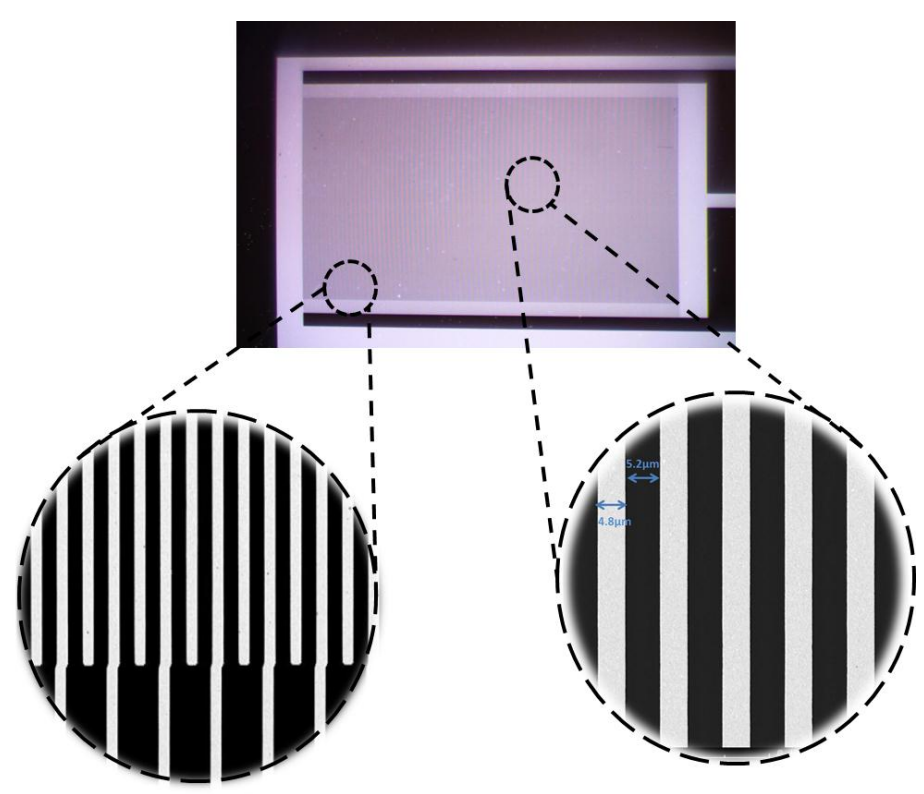

Fig 4.4 Microfabricated interdigitated electrode chip (Left); Microscopic image of the interdigitated fingers (Right)

\subsection{Fabrication of DTSP SAM}

Fig 4.5 presents a schematic illustration of the process of DTSP fabrication on the $\mathrm{Au}$ electrodes. Prior to fabrication, the microfabricated interdigitated gold electrodes were immersed in freshly prepared piranha solution followed by rinsing with DI water to clean the surface. The clean electrodes were next immersed in $2 \mathrm{mg} / \mathrm{ml}$ solution of DTSP in acetone followed by addition of Sodium borohydride to reduce the DTSP. The electrodes were kept immersed in the DTSP solution for SAM formation. Following SAM deposition, the DTSP-SAM/Au electrodes were rinsed with acetone and then by water to remove any unbound DTSP molecules. 


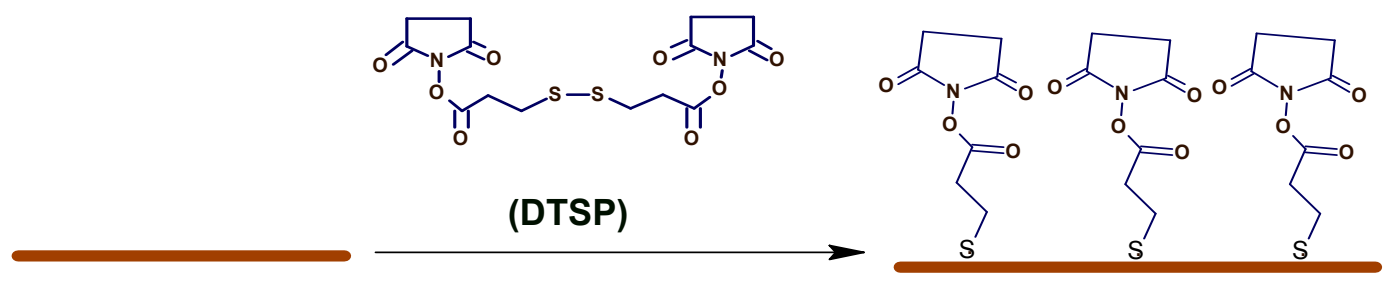

\section{Gold}

Fig 4.5 Schematic illustration of fabrication of DTSP-SAM on Au electrode.

\subsubsection{Electrochemical Characterization}

Results of electrochemical studies carried out using electrochemical impedance spectroscopy studies of the electrode and DTSP-SAM/Au electrode is presented in Fig 4.6. The Nyquist plot of bare Au electrode (curve a) shows a well-defined charge transfer resistance $\left(\mathrm{R}_{\mathrm{ct}}\right)$ correlated to electron transfer kinetics (corresponds to semicircle), and Warburg impedance correlated to diffusion process (straight line at $45^{\circ}$ to the axis). The

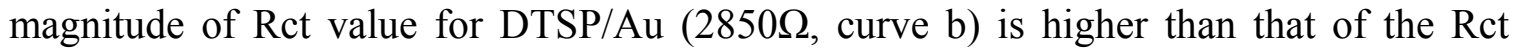

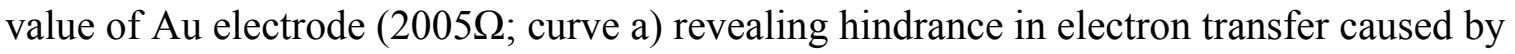
the insulating nature of DTSP-SAM and also confirm the fabrication of SAM onto Au electrode. The experimentally obtained data was simulated in an electronic equivalent circuit (inset). Similar trends were observed with $\mathrm{CV}$ measurements as well and are presented in the next section along with the biofunctionalization characterization. 


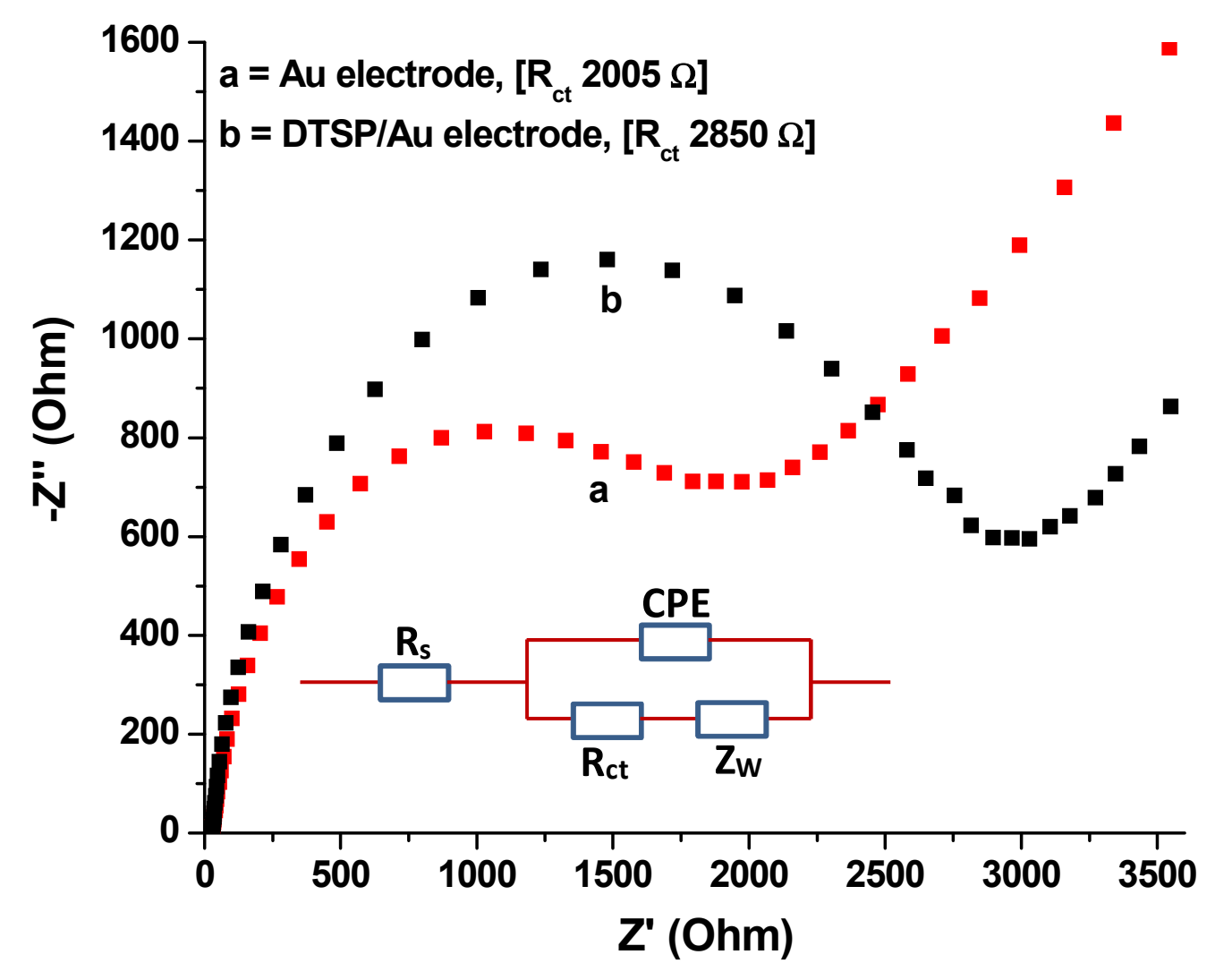

Fig 4.6 Electrochemical characterization of DTSP-SAM formation using electrochemical impedance spectroscopy (EIS)

\subsection{Functionalization of $\mu$ IDE for Cortisol Detection}

Next, the DTSP/Au electrode was functionalized with Anti-Cortisol antibodies. Fig 4.7 presents a schematic illustration of the biofunctionalization of the interdigitated microelectrodes. $10 \mu \mathrm{l}$ of Anti-cortisol antibodies (Anti-Cab) was incubated on the DTSP/Au electrode for $2 \mathrm{~h}$ for immobilization followed by carefully washing with PBS $(\mathrm{pH} 7.4,10 \mathrm{mM})$ to remove any unbound antibodies. Anti-Cab bind covalently (amide bond) via a facile reaction between amino group of antibody and reactive succinimidyl group of the DTSP SAM surface. The $\mathrm{NH}_{2}$ group of the DTSP-SAM binds with the 
carboxyl group $\left(\mathrm{F}_{\mathrm{c}}\right.$, terminal) of the Anti-Cab and the presence of free $\mathrm{NH}_{3}$ groups of Anti-Cab ( $\mathrm{F}_{\mathrm{ab}}$, terminal) leads to form a strong immunocomplex with cortisol. Ethanolamine $(10 \mu \mathrm{L})$ was immobilized for $10 \mathrm{~min}$ on to the Anti-Cab/DTSP/Au bioelectrode to block unreacted succinimidyl group on DTSP. The fabricated bioelectrodes were stored under refrigeration at $4^{\circ} \mathrm{C}$ when not in use.

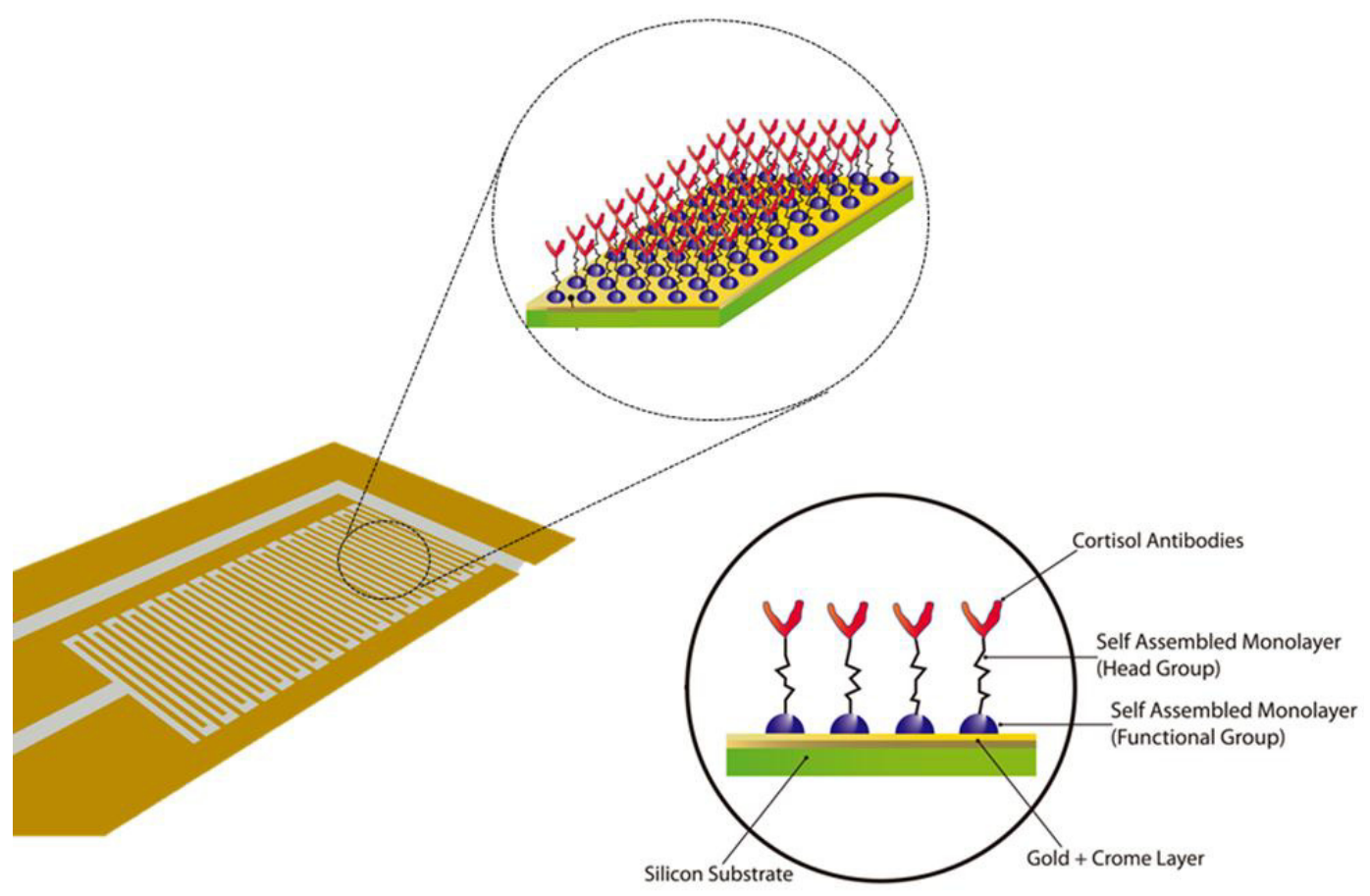

Fig 4.7 Schematic of $\mu$ IDE functionalization with Anti-Cortisol antibodies

The electrochemical behavior of the stepwise fabrication of DTSP/Au electrode and EA/Anti-Cab/DTSP/Au immunoelectrode has been studied using cyclic voltammetry (CV) techniques. The CV curves on the bare Au electrode (curve a), DTSP/Au electrode (curve b), Anti-Cab/DTSP/Au immunoelectrode (curve c), and EA/Anti-Cab/DTSP/Au immunoelectrode (curve d) have been carried out in PBS $(50 \mathrm{mM}, \mathrm{pH} 7.4$ containing $\left.5 \mathrm{mM}[\mathrm{Fe}(\mathrm{CN}) 6]^{3}-^{14}-\right)$ as shown in Fig 4.8. 


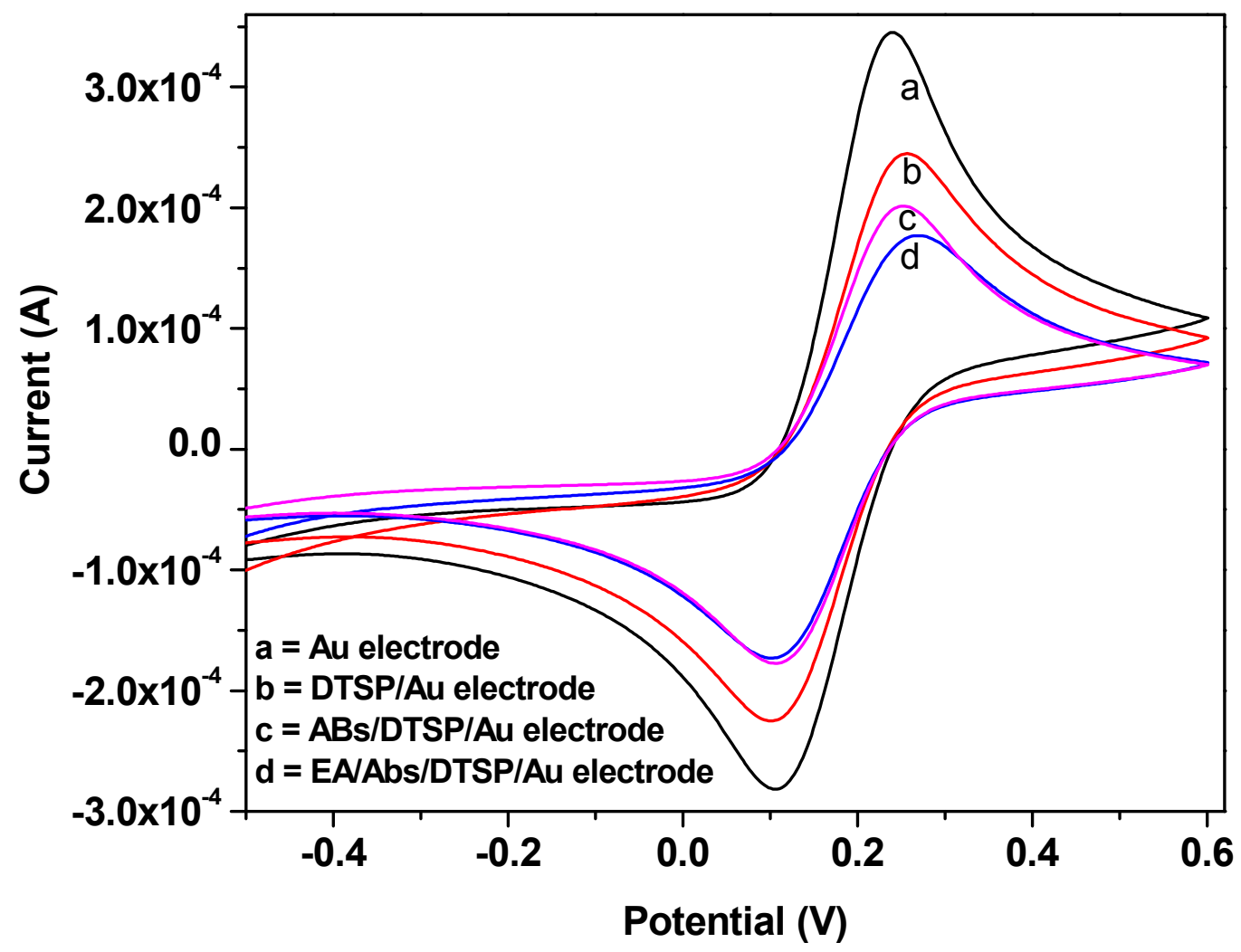

Fig 4.8 The CV curves on the bare Au electrode (curve a), DTSP/Au electrode (curve b), Anti-Cab/DTSP/Au immunoelectrode (curve c), and EA/Anti-Cab/DTSP/Au immunoelectrode (curve d) in have been carried out in PBS $(50 \mathrm{mM}, \mathrm{pH} 7.4$ containing $\left.5 \mathrm{mM}[\mathrm{Fe}(\mathrm{CN}) 6]^{3}-{ }^{4}-\right)$.

It is observed that the $\mathrm{Au}$ electrode shows very well-defined oxidation and reduction behavior of redox moieties $\mathrm{Fe}(\mathrm{II}) / \mathrm{Fe}$ (III) in the electrolyte. The magnitude of electrochemical response current of the Au electrode was found to decrease after the deposition of DTSP-SAM onto Au (curve b). This suggests the formation of DTSP-SAM, and due to the insulating nature of DTSP-SAM, electron transfer from the medium to $\mathrm{Au}$ electrode is hindered. After the immobilization of Anti-Cab onto the DTSP/Au electrode, the magnitude of the electrochemical response current further decreased (Curve c). This 
suggests that successful binding of Anti-Cab further hinders the electrons transport from medium to electrode. Furthermore, the magnitude of current response decreases after the immobilization of EA onto the Anti-Cab/DTSP/Au (Curve d) immunoelectrode due to blocking of non-specific binding sites of Anti-Cab that insulate the electrode and perturb electron communication.

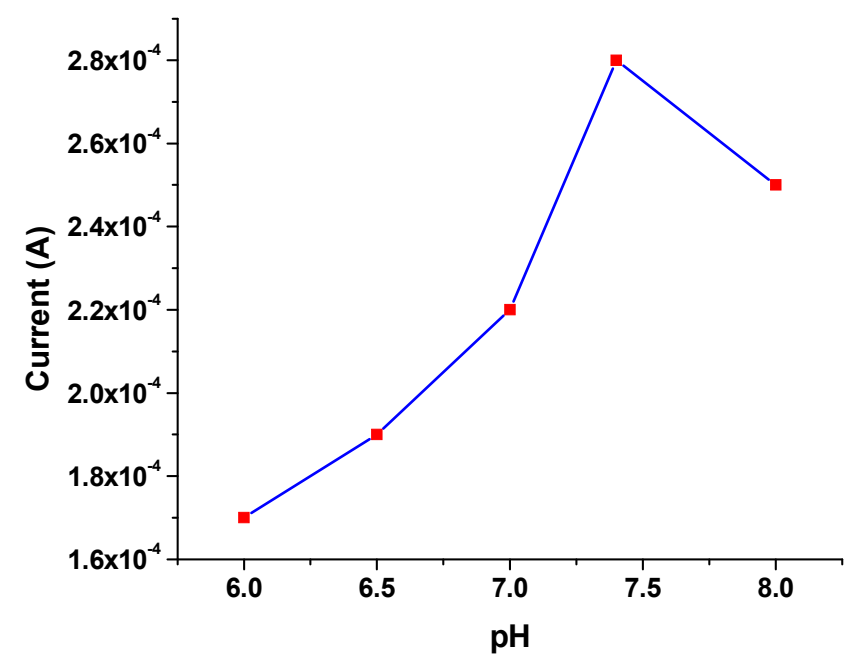

Fig 4.9 Electrochemical optimization as a function of $\mathrm{pH}$

$\mathrm{CV}$ studies have also been carried out to optimize the $\mathrm{pH}$ of PBS solution for electrochemical measurements (Fig 4.9). The magnitude of current response is $\mathrm{pH}$ dependent and maximum current response is observed at $\mathrm{pH}$ 7.4. Thus, $\mathrm{PBS}$ of $\mathrm{pH} 7.4$ has been used for electrochemical studies, which is the recommended $\mathrm{pH}$ to get immuno complex of high biological activity.

CV studies of DTSP/Au electrode (Fig 4.10a) and Anti-Cab/DTSP/Au immuno electrode (Fig 4.10b) were also carried out as a function of scan rate $(20-200 \mathrm{mV} / \mathrm{s})$. The magnitude of electrochemical current response and potential of both the electrodes are found linearly dependent to square root of scan rate and scan rate respectively revealing that diffusion of electrons onto electrode is a controlled process with facile electron 
transport. The surface concentration of redox moieties onto DTSP/Au and Anti$\mathrm{Cab} / \mathrm{DTSP} / \mathrm{Au}$ immunoelectrode have been estimated as $3 \times 10^{-6} \mathrm{~mol} / \mathrm{cm}^{2}$ and $1.5 \times 10^{-6}$ $\mathrm{mol} / \mathrm{cm}^{2}$, respectively using the Laviron equation. This suggests that larger numbers of redox moieties are available at the surface of DTSP/Au electrode for oxidation leading to a higher faradic current. This leads us to believe that uniformly distributed SAM provides an electronically operational surface for the immobilization of Anti-Cab through amide binding.

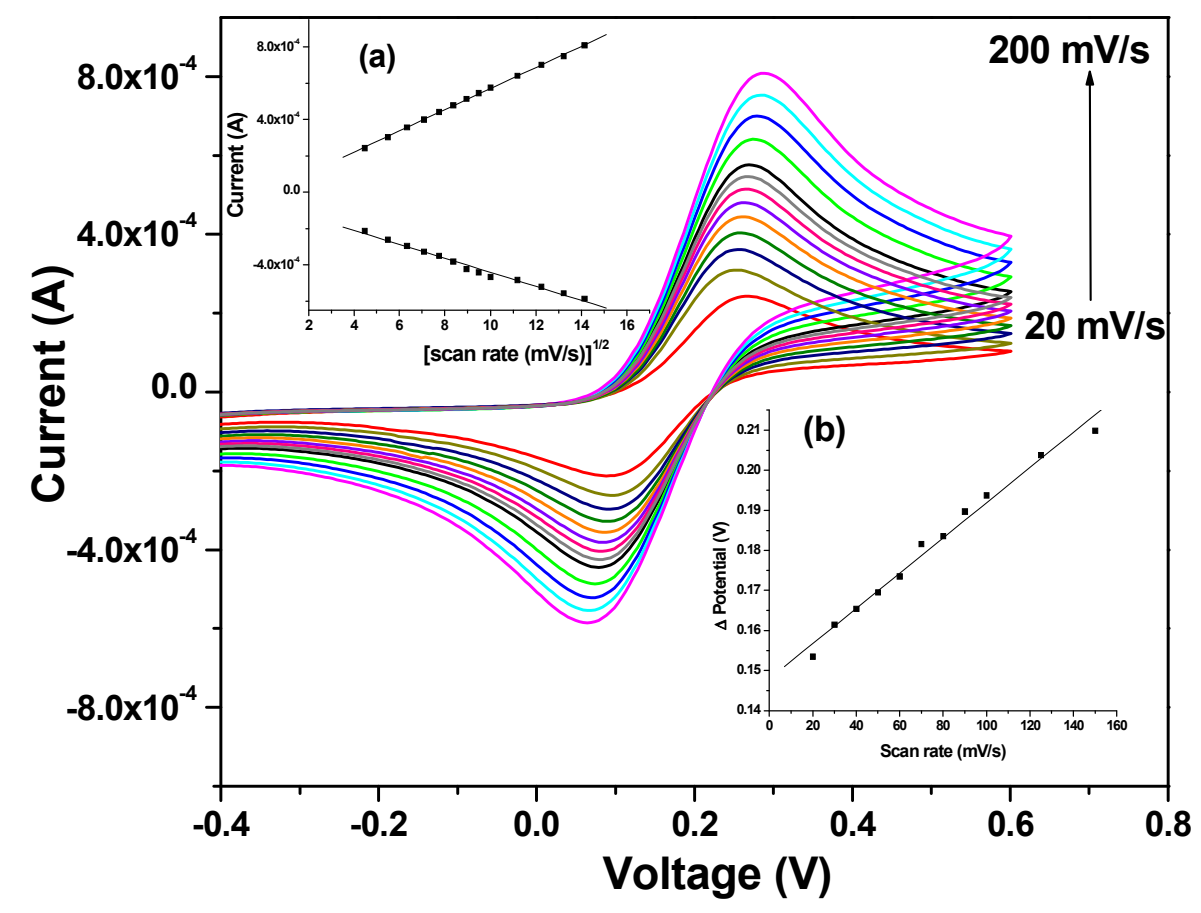




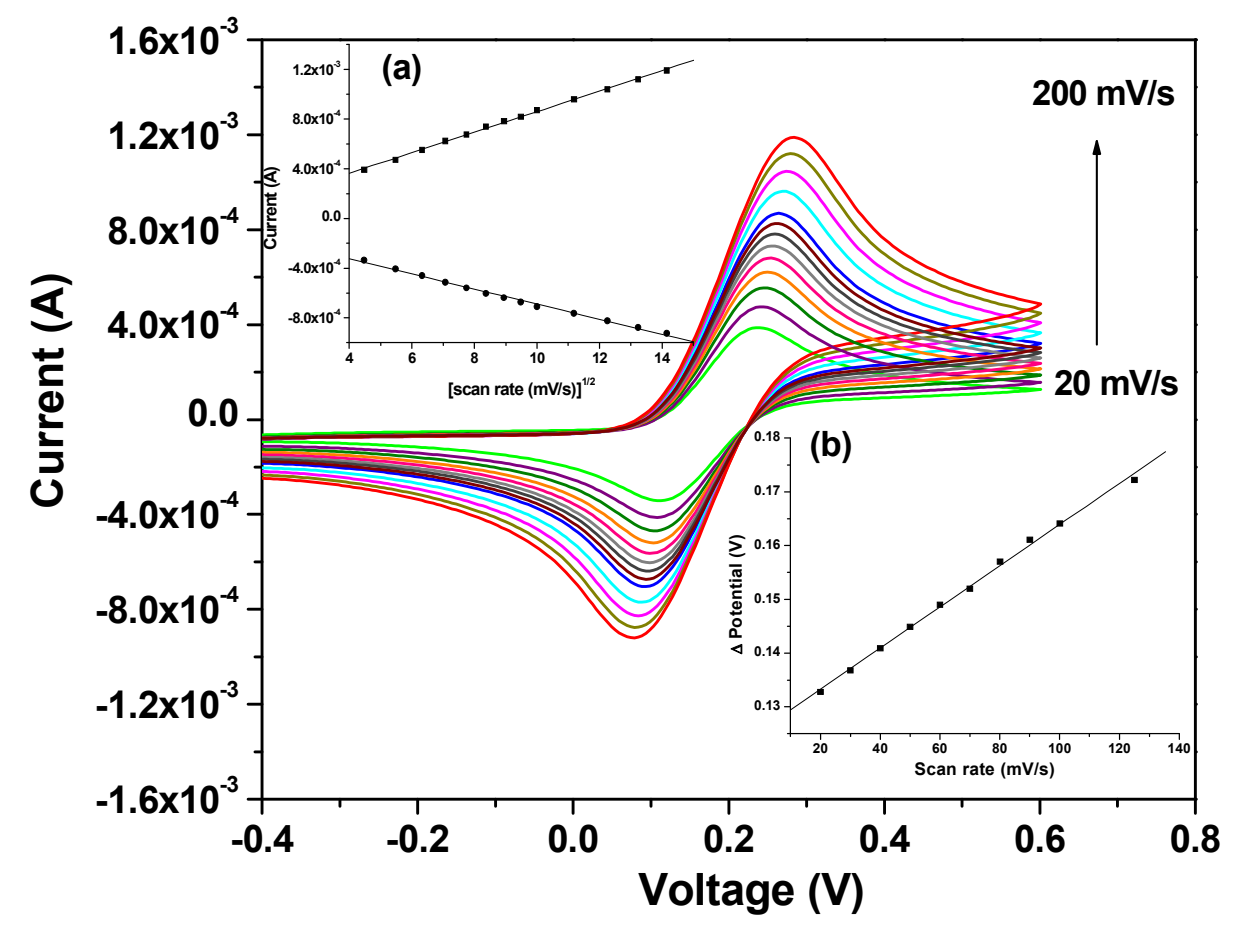

Fig.10. a) CV studies of DTSP/Au electrode as a function of scan rate (20-200 $\mathrm{mV} / \mathrm{s}$ ); inset a) magnitude of current response as a function of square root of scan rate, inset b) potential difference versus scan rate. b) CV studies of Anti-Cab/DTSP/Au immunoelectrode as a function of scan rate $(20-200 \mathrm{mV} / \mathrm{s})$; inset a) magnitude of current response as a function of square root of scan rate; inset b) potential difference versus scan rate. 


\subsection{Electrochemical Immunosensing Studies of Cortisol}

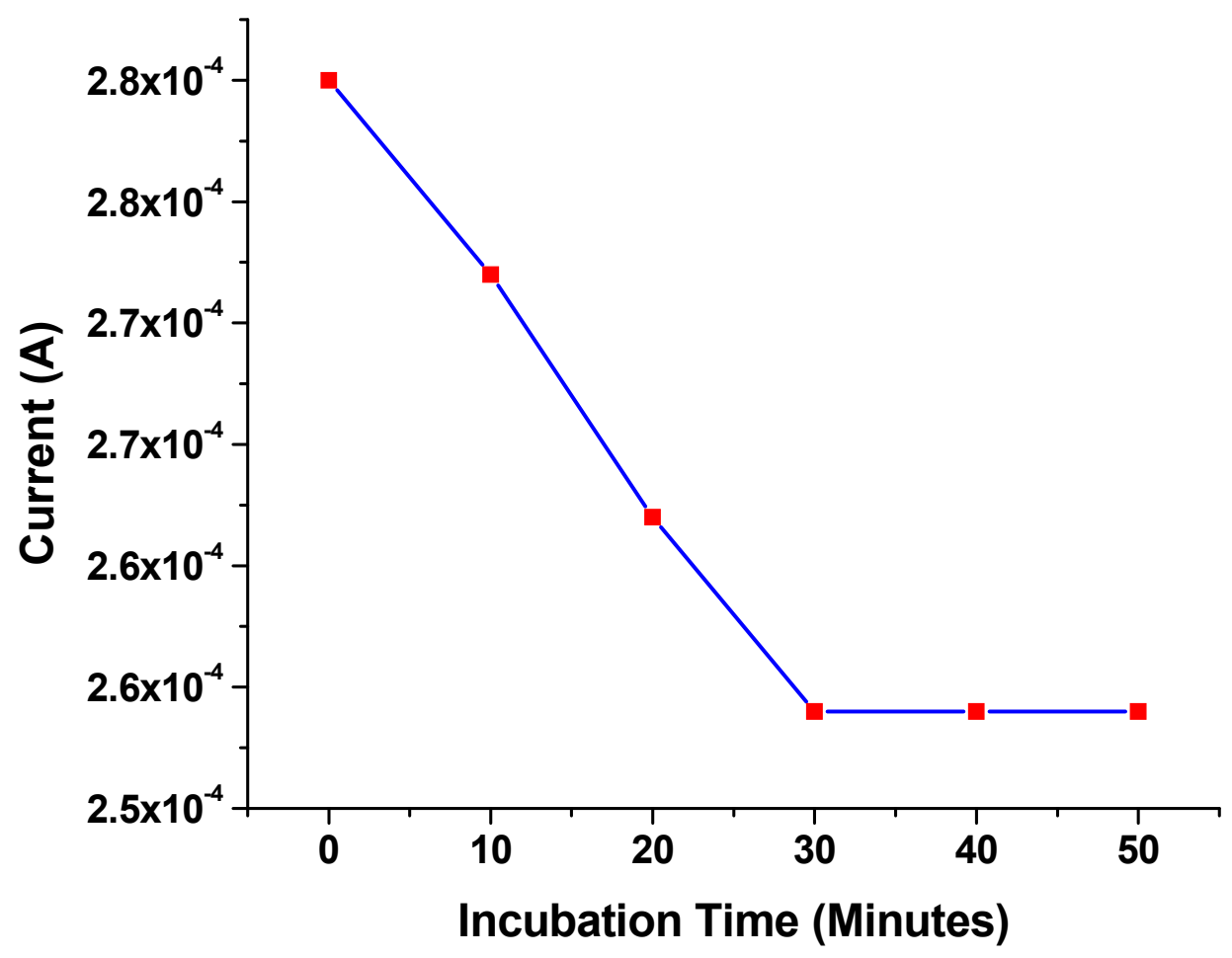

Fig 4.11 EA/Anti-Cab/DTSP/Au electrode incubated with $10 \mu \mathrm{L}$ of $200 \mathrm{nM}$ Cortisol.

Prior to carrying out sensing measurement, incubation time for complete binding between Cortisol and Anti-Cab has been optimized using CV (Fig 4.11). The magnitude of response current is dependent on electron transfer kinetics; hence to obtain a consistent and precise response current, the optimization of incubation time is necessary. CV measurements were performed at intervals of 10 minutes after incubation. The magnitude of response current decreases upon increasing the incubation time due to increased binding between Cortisol and Anti-Cab, resulting in increased electron transfer resistance. A consistent response current was obtained after incubation time of $30 \mathrm{~min}$. This time is consistent with literature reported for antigen-antibody binding kinetics, which suggests that more than $80 \%$ of the binding is completed in the first 30 minutes, after which the binding kinetics are minimal. 
The electrochemical response of EA/Anti-Cab/DTSP/Au immunoelectrode (Fig 4.12a) has been studied as a function of cortisol concentration (10 pM to $200 \mathrm{nM})$ using $\mathrm{CV}$ technique under identical experimental conditions (PBS (10 mM, pH 7.4) containing $5 \mathrm{mM} \mathrm{Fe}(\mathrm{CN})_{6}{ }^{3-/ 4-}$ as a redox probe and potential ranging from $-0.6 \mathrm{~V}$ to $\left.+0.6 \mathrm{~V}\right)$.

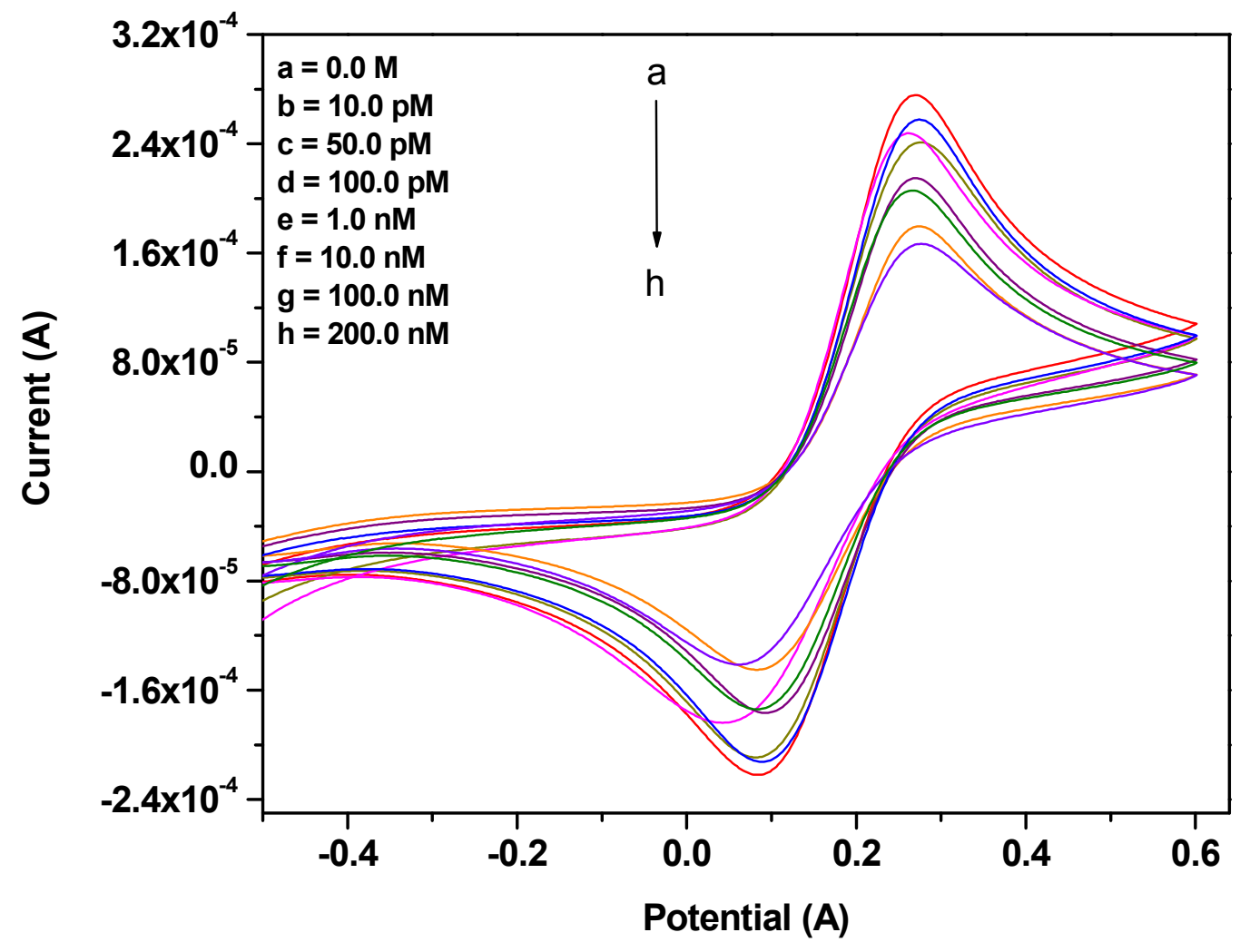




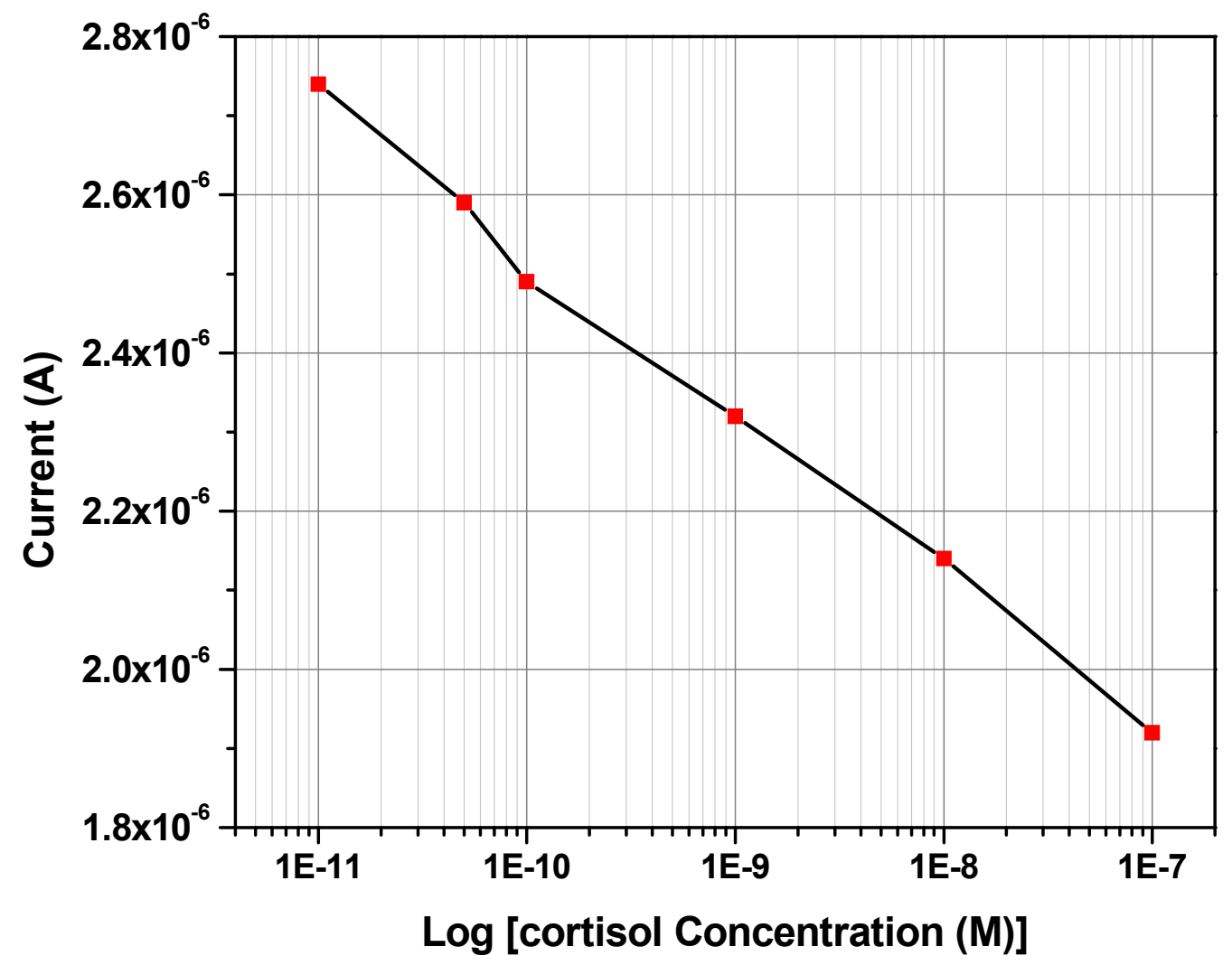

Figure 4.12 (a) Electrochemical response studies of EA/Anti-Cab/DTSP/Au immunoelectrode as a function of cortisol concentration (10pM to 200nM); (b) Calibration curve between magnitude of response current and log [cortisol concentration (M)].

It is can be seen from Fig 4.12a that the electrochemical response current decreases with increasing cortisol concentrations $(10 \mathrm{pM}-200 \mathrm{nM})$. This is due to the formation of immuno complexes between Anti-Cab and cortisol resulting in electron charge transfer hindrance at the electrode electrolyte interface. The magnitude of electrochemical response current of EA/Anti-Cab/DTSP/Au immunoelectrode is linearly dependent to the logarithm of cortisol concentration (Fig 4.12b) and obeys the relation $\boldsymbol{Y}$ 
$=A\left(4.4 \times 10^{-5}\right)-2.0710^{-5} \times \log ($ Cortisol Concentration); $R=0.997$. The EA/Anti$\mathrm{Cab} / \mathrm{DTSP} / \mathrm{Au}$ immunoelectrode exhibits linear range from $10 \mathrm{pM}$ to $200 \mathrm{nM}$, at a detection limit of $10 \mathrm{pM}$ and sensitivity of $20.7 \mu \mathrm{A} \mathrm{M}^{-1}$ with a correlation coefficient of 0.997 (SD: $0.78 \mu \mathrm{A})$. The value of the association constant $\left(K_{\mathrm{a}}\right)$ was estimated at $9 \times 10^{11}$ $\mathrm{L} / \mathrm{mol}$ indicating strong affinity of Anti-Cab with cortisol attributed to the immobilization of Anti-Cab onto the DTSP/Au electrode in the desired orientation. To determine the specificity of the immunosensor towards Cortisol and the effect of non-specific adsorption, electrochemical response was measured in the presence of other commonly present antigens. The EA/Anti-Cab/DTSP/Au immunoelectrode showed a minimum change in electrochemical response in the presence of PSA (200nM), EGFR (200nM) and NSE (200nM). However, a significant change in electrochemical response was obtained for cortisol (200nM) and Cortisol mixed with BSA (150nM Cortisol + 50nM BSA), confirming that immunoelectrode is specific to Cortisol with minimal signal from nonspecific antigens. 


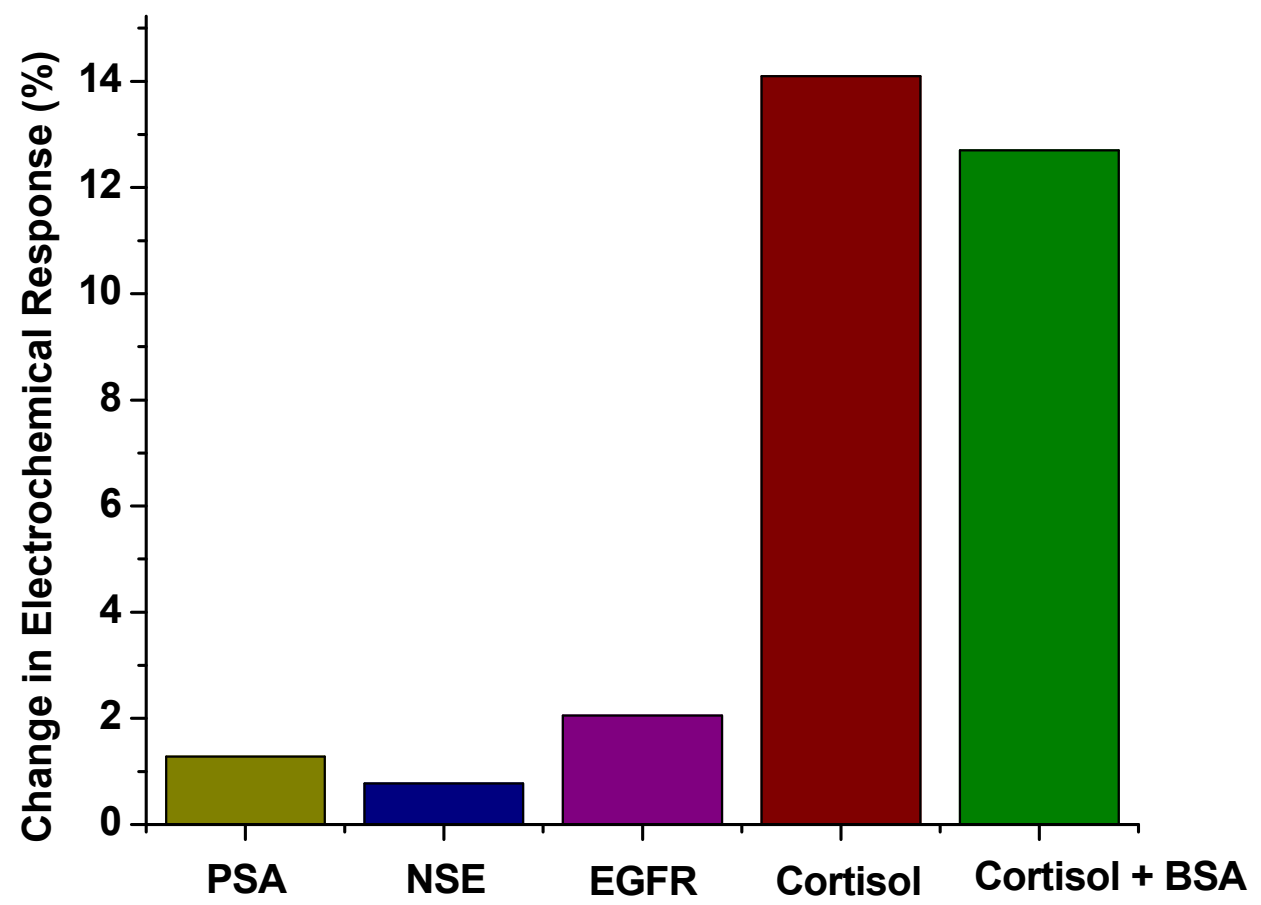

Fig 4.13 Percentage change in electrochemical response of the EA/Anti-Cab/DTSP/Au immunoelectrode after incubation with PSA (200nM), EGFR (200nM), NSE (200nM), Cortisol (200nM) and Cortisol mixed with BSA (150nM Cortisol + 50nM BSA).

\subsection{Cortisol Detection in Saliva Samples}

The developed DTSP self-assembled monolayer based electrochemical immunosensor for cortisol sensing was utilized for testing of cortisol concentrations in saliva samples. Saliva samples were obtained from Mexican immigrant farm workers in collaboration with Dr. Shedra Amy Snipes, assistant professor of behavioral health, Pennsylvania State University, who utilizes the saliva sample to detect cortisol concentrations and correlate it to organophosphate exposure to farm workers in the field. The saliva samples were de-identified by stripping out all identifiers such as name, age, gender, etc. related to the source of the saliva samples. Normal values of cortisol 
concentration at different time zones, as defined by Mayo medical diagnostics, during a 24-hour time period is presented in Table 4.1.

\begin{tabular}{|c|c|}
\hline Time Zone & Normal Cortisol Concentration (ng/dL) \\
\hline 5 a.m.-10 a.m. & $100-750$ \\
\hline 3 p.m.-5 p.m. & $<401$ \\
\hline 11 p.m.-midnight & $<100$ \\
\hline
\end{tabular}

Table 4.1 Normal cortisol concentrations as defined by Mayo medical diagnostics.

The saliva samples were collected at two separate time period in a 24-hour span over three days using special saliva collection devices called salivettes. The obtained salivettes were stored at $-20 \mathrm{C}$ until used. Prior to usage, the saliva samples were defreezed to room temperature and centrifuged for 15 minutes at 3500rpm to harvest the saliva from the salivette. To perform the electrochemical detection, $10 \mu \mathrm{L}$ of the saliva sample was incubated on the electrochemical immunosensor for a period of 30 minutes. A washing step using $30 \mathrm{uL}$ of PBS was performed to remove the saliva and any unbound cortisol from the sensor surface. Electrochemical response studies were carried out using cyclic voltammetry in $10 \mu \mathrm{L}$ of PBS containing $5 \mathrm{mM} \mathrm{Fe}(\mathrm{II}) / \mathrm{Fe}(\mathrm{III})$ as the redox probe. All samples were measured in triplicate sets and the average was computed and is presented in Table 4.2. The standard curve obtained from known concentrations of cortisol was then used to determine the concentration of cortisol in the saliva samples. 


\begin{tabular}{|c|c|c|c|c|}
\hline Specimen & $\begin{array}{c}\text { Saliva Collection } \\
\text { Time and Date }\end{array}$ & $\begin{array}{c}\text { Measured } \\
\text { Electrochemical } \\
\text { Response }(\mu \mathrm{A})\end{array}$ & $\begin{array}{c}\text { Cortisol } \\
\text { Concentration } \\
\text { (Obtained } \\
\text { Concn. } x \text { 2.2) } \\
(\mathrm{nMol} / \mathrm{L})\end{array}$ & $\begin{array}{c}\text { Final Cortisol } \\
\text { Concentration } \\
(\mathrm{ng} / \mathrm{dL})\end{array}$ \\
\hline $\mathrm{P} 137$ & $3 / 26 / 2013(9: 47 \mathrm{AM}$ & 7.16 & 3.63 & 131.78 \\
\hline & $3 / 27 / 2013(10 \mathrm{AM})$ & 7.38 & 3.81 & 138.37 \\
\hline & $3 / 27 / 2013(10: 03 \mathrm{P}$ & 12.23 & 0.90 & 32.94 \\
\hline & $3 / 28 / 2013(2 \mathrm{AM})$ & 8.89 & 1.818 & 65.89 \\
\hline $\mathrm{P} 138$ & $3 / 26 / 2013(10: 13 \mathrm{AM})$ & 5.24 & 9.09 & 329.45 \\
\hline & $3 / 27 / 2013(1: 42 \mathrm{AM}$ & 7.76 & 2.727 & 98.83 \\
\hline & $3 / 27 / 2013(10: 13 \mathrm{~A})$ & 4.26 & 14.54 & 428.29 \\
\hline & $3 / 28 / 2013(3: 02 \mathrm{AM}$ & 9.17 & 1.93 & 69.18 \\
\hline
\end{tabular}

Table 4.2 Cortisol values measured in saliva samples using the developed electrochemical immunosensor

To validate the results obtained from the electrochemical immunosensor, saliva samples from the same salivette were tested for cortisol using ELISA (Arbor Assays, MI), the current gold standard for measuring cortisol concentrations in saliva samples. The protocol prescribed by the ELISA kit vendor was followed for cortisol detection in saliva samples. To establish the calibration curve for cortisol measurement, the assay was performed in a 96-well titer plate consisting of six known standard cortisol concentrations $(100,200,400,800,1600$ and $3200 \mathrm{pg} / \mathrm{mL})$ and the saliva samples. The calibration curve obtained from the measurement of the standard solutions is presented in Fig 4.14. Next, the saliva samples were diluted 1:4 in the provided assay buffer and 50uL of saliva samples were utilized for each measurement. Saliva samples from each of the salivettes were tested in triplicate sets and the average value was utilized to determine the final cortisol concentration and is presented in Table 4.3. 


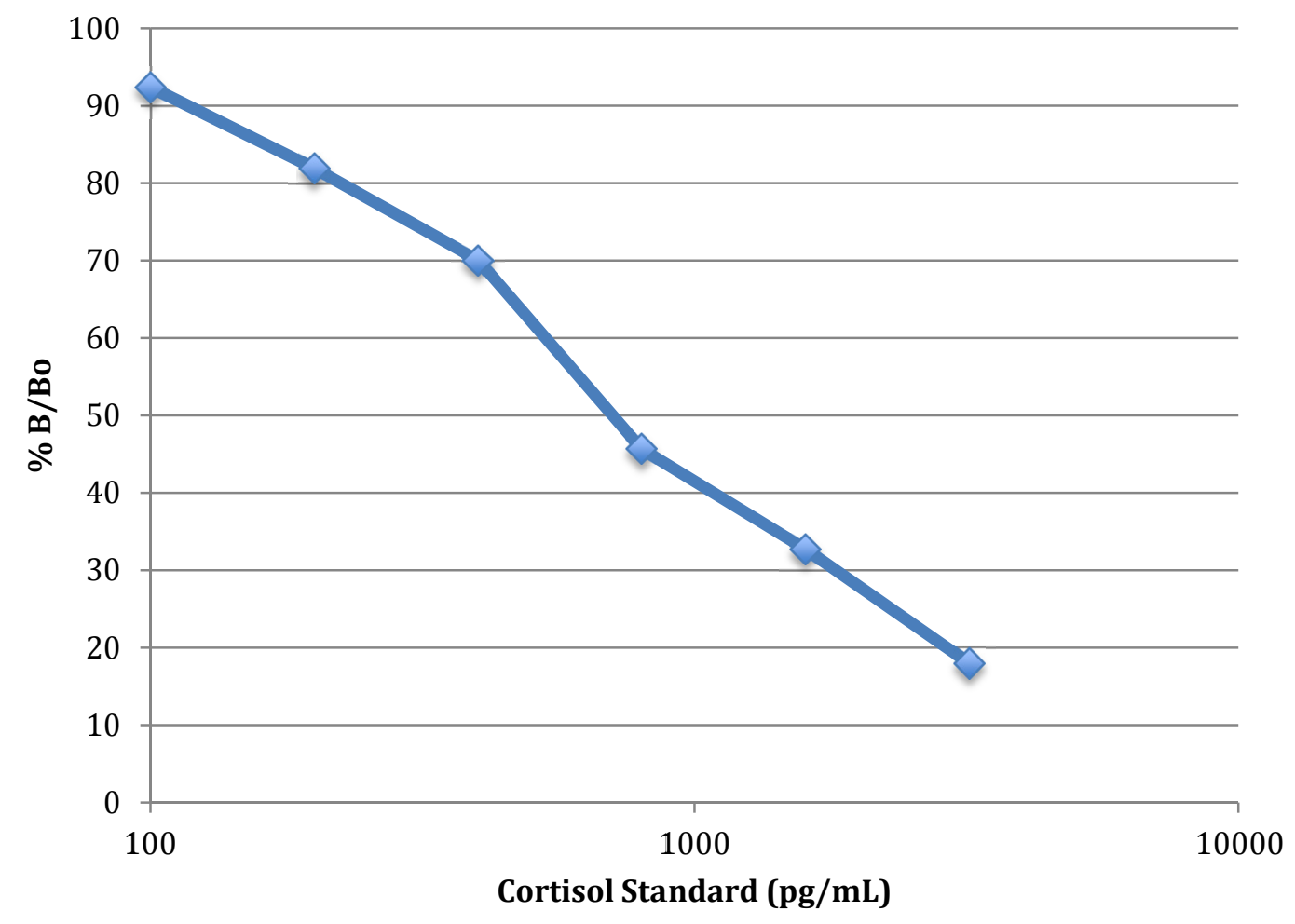

Fig 4.14 The standard curve obtained for determination of the cortisol concentrations in saliva samples using ELISA.

\begin{tabular}{|c|c|c|c|c|}
\hline Sample & $\begin{array}{c}\text { Saliva Collection } \\
\text { Time and Date }\end{array}$ & $\begin{array}{c}\text { Measured Response } \\
(4 \mathrm{x} \text { dilution) } \\
(\% \mathrm{~B} / \mathrm{Bo})\end{array}$ & $\begin{array}{c}\text { Cortisol } \\
\text { Concentration } \\
(\mathrm{pg} / \mathrm{mL}) \\
4 \mathrm{X} \text { dilution }\end{array}$ & $\begin{array}{c}\text { Final Cortisol } \\
\text { Concentration } \\
(\mathrm{ng} / \mathrm{dL})\end{array}$ \\
\hline P137 & $3 / 26 / 2013(9: 47 \mathrm{AM}$ & 64.133 & 389 & 155.6 \\
\hline & $3 / 27 / 2013(10 \mathrm{AM})$ & 66.909 & 360 & 144 \\
\hline & $3 / 27 / 2013(10: 03 \mathrm{P})$ & 56.113 & 594 & 237.6 \\
\hline P138 & $3 / 28 / 2013(2 \mathrm{AM})$ & 81.758 & 182 & 72.8 \\
\hline & $3 / 26 / 2013(10: 13 \mathrm{AM})$ & 41.705 & 995 & 398 \\
\hline & $3 / 27 / 2013(1: 42 \mathrm{AM})$ & 99.559 & 125 & 50 \\
\hline & $3 / 27 / 2013(10: 13 \mathrm{~A})$ & 40.956 & 1187 & 474.8 \\
\hline
\end{tabular}

Table 4.3 Cortisol values measured in saliva samples using the commercially available Cortisol ELISA kit. 
Fig 4.14 (a) and Fig 4.14 (b) presents the comparison of results obtained for cortisol detection in saliva samples tested using the electrochemical immunosensor and ELISA. The trend observed for the electrochemical measurements were mostly consistent with that obtained from ELISA, except for the saliva sample from Specimen 137 (3/27/2013 (10:03P)), in which the ELISA value was observed to be a high value at night time. All values of cortisol concentration obtained from the corresponding calibration curve were corrected by a factor of 2.2 in order to compare with values obtained from ELISA assays.

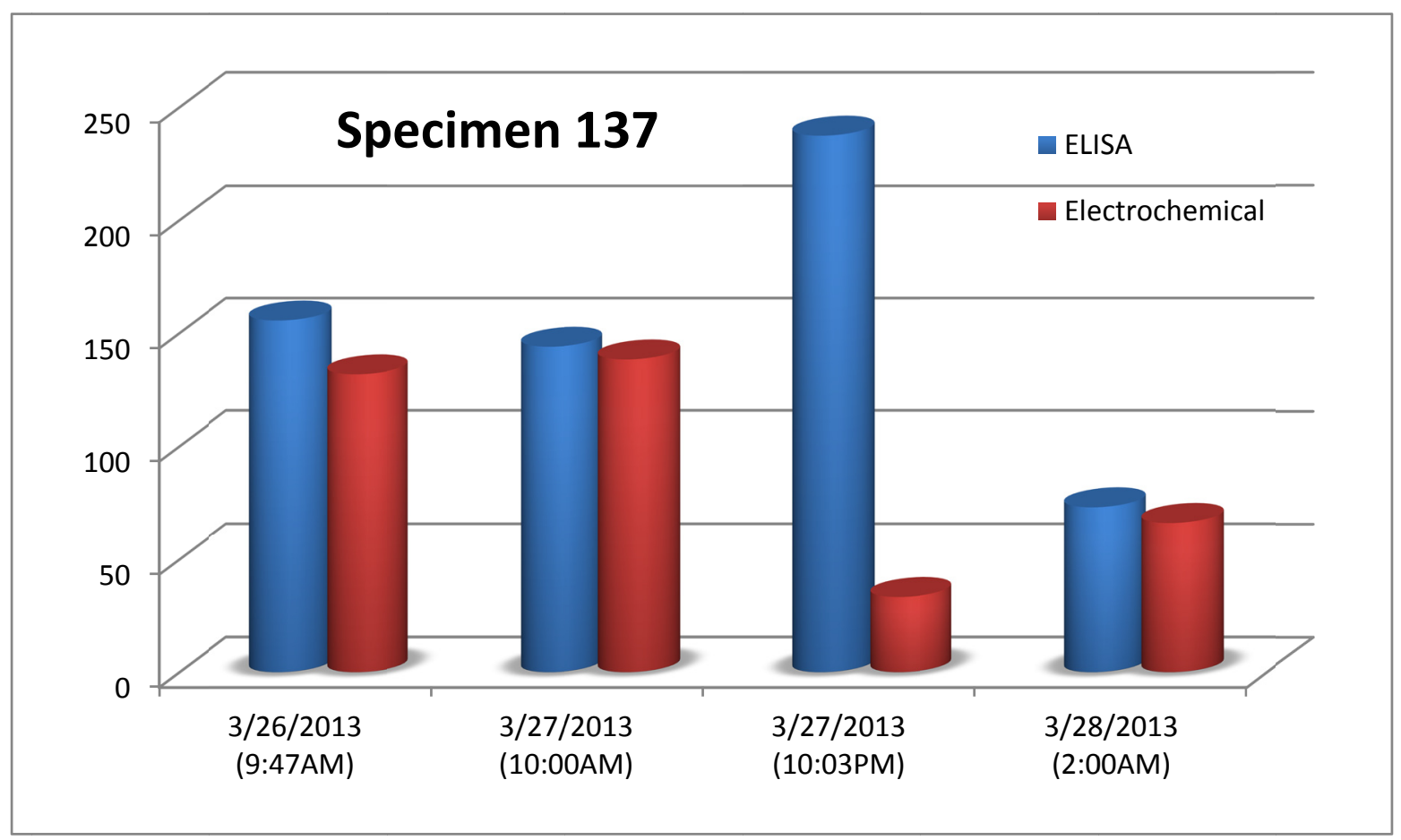




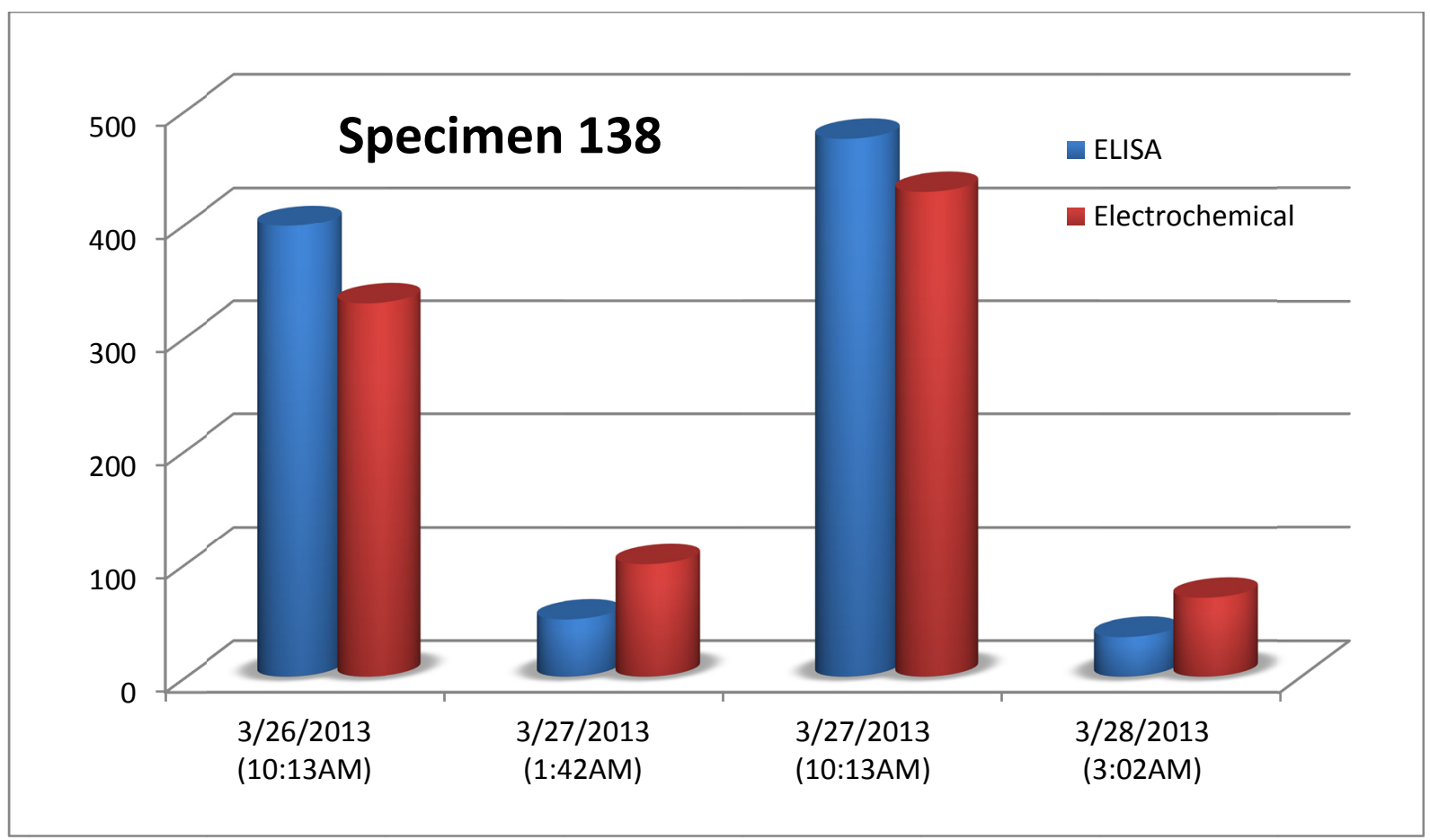

Figure 4.15 Comparison of cortisol concentration measured by ELISA and Electrochemical measurement on saliva samples from (a) Specimen 137 and (b) Specimen 138 at specific time intervals over three days.

Fig 4.14 (a) and Fig 4.14 (b) presents the comparison of results obtained for cortisol detection in saliva samples tested using the electrochemical immunosensor and ELISA. The trend observed for the electrochemical measurements were mostly consistent with that obtained from ELISA, except for the saliva sample from Specimen 137 $(3 / 27 / 2013(10: 03 \mathrm{P}))$, in which the ELISA value was observed to be a high value at night time. It was observed that the obtained values from the electrochemical measurements were consistent with the trend observed from the ELISA measurements, however at an off-set. Hence, all values of cortisol concentration obtained from the corresponding calibration curve were corrected by a factor of 2.2 in order to compare with values obtained from ELISA assays. A good correlation was observed between the two sets of 
data, thus enabling quantitative validation of the electrochemical immunosensing of Cortisol in a biologically relevant fluid such as saliva.

\subsection{Conclusion}

The research activity undertaken was to develop a DTSP-SAM based electrochemical immunosensing platform for the detection of cortisol. The DTSP-SAM was modified onto microfabricated interdigitated microelectrodes ( $\mu \mathrm{IDE}$ ). Electrochemical characterization of the DTSP-SAM modification and fabrication of the immunoelectrode is presented. The immunosensor was tested as a function of cortisol concentrations in phosphate buffer saline (PBS) using cyclic voltammetry and found to exhibit a detection limit of $10 \mathrm{pM}$, a linear range from $10 \mathrm{pM}$ to $200 \mathrm{nM}$, with a sensitivity of $20.7 \mu \mathrm{A} \mathrm{M}^{-1}$. The next research activity planned was to automate the electrochemical immunoassay as a step towards realizing a fully automated point-of-care cortisol sensor. The next chapter details the efforts to integrate the developed DTSP-SAM based cortisol immunosensor on the $\mu$ IDE's into a low-cost, disposable microfluidic system. 


\subsection{References}

1. Wink, T., et al., Self-assembled monolayers for biosensors. Analyst, 1997. 122(4): p. 43R-50R.

2. Arya, S.K., et al., Recent advances in self-assembled monolayers based biomolecular electronic devices. Biosensors and Bioelectronics, 2009. 24(9): p. 2810-2817.

3. Hahn, C.D., et al., Self-assembled monolayers with latent aldehydes for protein immobilization. Bioconjugate chemistry, 2007. 18(1): p. 247-253.

4. Jung, S.-H., et al., Oriented immobilization of antibodies by a self-assembled monolayer of 2-(biotinamido) ethanethiol for immunoarray preparation. Colloids and Surfaces B: Biointerfaces, 2006. 47(1): p. 107-111.

5. Chan, E.W. and M.N. Yousaf, Immobilization of ligands with precise control of density to electroactive surfaces. Journal of the American Chemical Society, 2006. 128(48): p. 15542-15546.

6. Shervedani, R.K. and A. Hatefi-Mehrjardi, Electrochemical characterization of directly immobilized glucose oxidase on gold mercaptosuccinic anhydride selfassembled monolayer. Sensors and Actuators B: Chemical, 2007. 126(2): p. 415423.

7. Mizutani, F., Biosensors utilizing monolayers on electrode surfaces. Sensors and Actuators B: Chemical, 2008. 130(1): p. 14-20.

8. Matemadombo, F., et al., Immobilization of tetra-amine substituted metallophthalocyanines at gold surfaces modified with mercaptopropionic acid or DTSP-SAMs. Electrochimica Acta, 2007. 52(5): p. 2024-2031.

9. Matharu, Z., et al., Self-assembled monolayer for low density lipoprotein detection. Journal of Molecular Recognition, 2008. 21(6): p. 419-424.

10. Bartsch de Torres, H., et al., Thick film flow sensor for biological microsystems. Sensors and Actuators A: Physical, 2010. 160(1): p. 109-115.

11. Varshney, M. and Y. Li, Interdigitated array microelectrodes based impedance biosensors for detection of bacterial cells. Biosensors and Bioelectronics, 2009. 24(10): p. 2951-2960.

12. Manso, J., et al., Electrochemical biosensors based on colloidal gold-carbon nanotubes composite electrodes. Journal of Electroanalytical Chemistry, 2007. 603(1): p. 1-7. 
13. Van Gerwen, P., et al., Nanoscaled interdigitated electrode arrays for biochemical sensors. Sensors and Actuators B: Chemical, 1998. 49(1): p. 73-80.

14. Zou, Z., et al., Functionalized nano interdigitated electrodes arrays on polymer with integrated microfluidics for direct bio-affinity sensing using impedimetric measurement. Sensors and Actuators A: Physical, 2007. 136(2): p. 518-526.

15. Min, J. and A.J. Baeumner, Characterization and optimization of interdigitated ultramicroelectrode arrays as electrochemical biosensor transducers. Electroanalysis, 2004. 16(9): p. 724-729. 


\section{CHAPTER V}

\section{Low Temperature Co-fired Ceramics (LTCC) Based Microfluidic System for Automation of Cortisol Immunoassay}

In this chapter, the design, fabrication and fluidic characterization of LTCC based microfluidic system is presented. The optimization of the microfluidic system design for the automation of the immunoassay has been done using a computational fluid dynamic approach. A novel approach using LTCC technology has been applied for the fabricating a microfluidic system. For the first time, the non-specific adsorption (NSA) of a biomolecule (Cortisol) on LTCC surface has been characterized as part of the microfluidic system design and development.

\subsection{Non-Specific Adsorption (NSA) of Cortisol on LTCC}

Advantages such as rapid prototyping, 3D architecture, and cost effective fabrication have made LTCC an attractive alternative to polymer, silicon and glass based microfluidics. With a steady increase in the application of LTCC microfluidic systems for sensing of biomolecules, a thorough characterization of LTCC as a material for biomicrofluidic systems is imperative. An important material property that is taken into account during microfluidic device development is the biocompatibility of the utilized materials that interact with the bio-analyte[1]. The term biocompatibility, in general, encompasses several features such as host-tissue response, immune and inflammatory response, toxicity etc., which are however more pertaining to in-vivo scenarios. For invitro applications, biocompatibility is measured by factors such as facilitation of cell 
cultures in scaffold materials for tissue engineering, material disintegration during material-fluid interaction, and the non-specific adsorption of proteins, enzymes, DNA and other biomolecules onto the material surface.

To assess the biocompatibility of LTCC, Smetana et al. [2] have reported the characterization of cell proliferation of various cell lines (HeLa cells, BAC cells and L929 cells) on three commercially available LTCC materials in both the fired as well as green state. The results indicate that apart from the CeramTec tapes, all other LTCC tapes showed cell proliferation comparable to the control (glass). Cell culture chambers in LTCC microfluidic devices are often integrated with electronic sensors and actuators, typically achieved through screen-printed metals. Hence, the understanding of the effect of metal compositions on cellular responses is imperative for design considerations. During the process of development of LTCC based flow sensor for biological applications, Torres et al. [3] have reported the investigation of cell culture growth (mammalian cell lines HEK 293 FT and CHO-k1) on LTCC substrates. Cellular response studies were carried out on both LTCC blank substrates (DuPont 951) as well as screenprinted metal compositions (DuPont Au, Pt, Ag). Additionally PCR experiments were also carried out in the presence of LTCC blanks and the screen print metal compositions. The results indicate that the LTCC blanks provide a congenial surface for cellular activities; however, a negative cellular response was observed on the screen-printed metal compositions. Leachates from the metal have been singled out as the reason for the detrimental effects on cell cultures. Recently, Mercke et al. [4] have evaluated the viability of culturing HUVEC (Human Umbilical Vein Endothelial Cells) on Heralock ${ }^{\circledR}$ HL2000 tapes along with their recommended screen printed Au and Ag paste. While their 
results indicate difficulty in initial attachment of the cells onto the LTCC substrate, no negative response was observed for cells cultured on the screen-printed films of $\mathrm{Au}$ and Ag. The cell cultures showed good viability up to three days. Recently, Zhang et al[5] have reported a thorough characterization of the biostability of LTCC material for biomedical and microfluidic device applications. Leaching rate studies of three commercially available green tape materials have been studied during LTCC interaction with relevant fluids such as phosphate buffer saline (PBS), simulated body fluid (SBF), simulated gastric fluid (SGF), and a basic solution (1M NaOH solution, no physiological analogue). Leaching rate of LTCC in these fluids has been determined through weight loss and elemental analysis. The reported results suggest that techniques such as surface passivation, flushing out of leached material and diffusion limited leaching may be required depending on the LTCC-fluid exposure time.

When microfluidic devices are used for biosensing applications, a factor of importance is the interaction of the analyte with the surface of the microchannels and the assay chamber. A common phenomenon that is observed is the rapid adsorption of biomolecules (analyte) on the surface of the device[6]. This phenomenon, referred to as non-specific adsorption (NSA), can lead to loss of analyte concentration via adsorption on to the surface leading to erroneous results[7]. An understanding of NSA is especially important in microfluidic biosensors which involve incubation steps for considerable lengths of time. The NSA of biomolecules is commonly observed in microfluidic systems fabricated from PDMS[6], PMMA[8], Silicon[9] and Glass[10]. Information obtained from NSA studies is used to develop surface modification techniques to reduce NSA. Many surface pretreatment strategies such as coating, plasma exposure, hydrophobic 
tuning and decreasing surface charge are well characterized to reduce non-specific adsorption on microfluidic materials such as silicon, glass, and polymers[11].

With increasing application of LTCC based microfluidic systems for biosensing applications, the characterization of NSA of biomolecules on LTCC has become essential.

\subsubsection{Design of Experiment, Materials and Methods}

All the characterization has been performed on DuPont 951 LTCC tapes. The unfired samples were used as received and the fired LTCC samples were obtained by sintering the samples in an oxygen rich furnace to $850^{\circ} \mathrm{C}$ at a temperature ramp profile as shown in Fig. 1. Scanning electron microscopy (SEM) was performed on unfired and fired LTCC tapes using a tabletop SEM (Phenom, Netherlands).

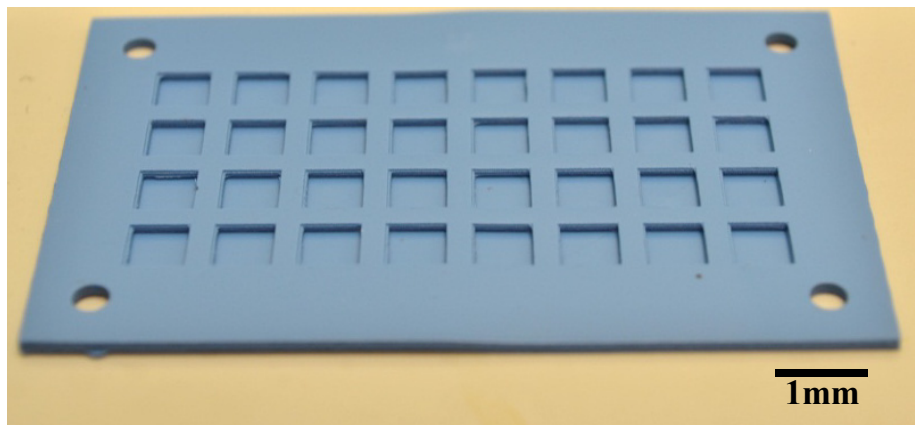

Fig 5.1 LTCC 32 micro-well plate fabricated for the NSA characterization.

X-Ray Diffraction (XRD) analysis was performed on the LTCC samples using a Siemens Diffraktometer. The X-ray patterns were measured from 2theta ranging from $10^{\circ}$ to $70^{\circ}$ at a rate of $2 \mathrm{deg} / \mathrm{min}$. For XRD analysis performed on Cortisol incubated LTCC samples, $50 \mu \mathrm{L}$ of Cortisol solution of $500 \mathrm{nM}$ concentration in PBS was incubated on fired LTCC samples for a period of 1 hour and allowed to dry at room temperature (unwashed). Contact angle measurements were performed using a contact angle 
goniometer (ramé-hart, $\mathrm{NJ}$ ) using $0.5 \mu \mathrm{L}$ of DI water (surface tension: $72 \mathrm{dyn} / \mathrm{cm}$ ) as the test liquid.

For the quantitative analysis of non-specific adsorption of cortisol on LTCC, Enzyme-Linked Immunosorbent Assay (ELISA) has been utilized. $5 \mu \mathrm{L}$ of two prepared concentrations of Cortisol in phosphate buffer saline $(1 \mu \mathrm{M}$ and $500 \mathrm{nM})$ were pipetted on to LTCC surface and incubated for increasing time periods. After specific time periods the solution was removed and cortisol concentration assays were performed using commercially available Cortisol ELISA kits (Arbor Assays, Michigan). The concentration of residual Cortisol in the solution was used to quantify the amount of NSA. To reduce variability due to sample preparation and testing conditions, adsorption studies were carried out in 32-well plates (Fig 5.2), fabricated on a $4 \mathrm{~cm} \times 6 \mathrm{~cm}$ substrate using standard LTCC processing techniques. The adsorption tests were run in duplicates and the ELISA tests were further run in triplicates. The volume in each well was designed for $10 \mu \mathrm{L}$ by optimizing the number of LTCC layers.

\subsubsection{Results and Discussion}
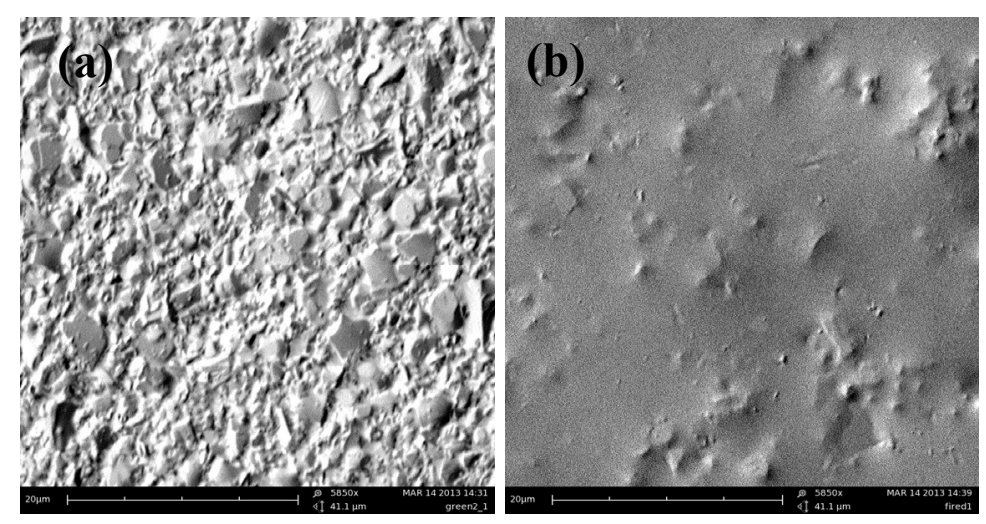

Fig 5.2 Scanning electron microscope images of the DuPont 951 LTCC green tapes in the (a) Unfired and (b) Fired state. 
Fig 5.2 presents the SEM images of the DuPont 951 LTCC tapes in the green (Fig 5.2a) and the fired state (Fig 5.2b). The highly granular structure visible in the green state represents the micro particles of Alumina and glass suspended in an organic matrix. It can be seen that the morphology of the surface changes to a much less abrasive appearance in the fired tapes due to the burnout of the organic components and the encapsulation of the ceramic granules with the melted glass. This was validated through characterization of the surface roughness of the unfired and fired LTCC substrates. Fig $5.3(a, b)$ presents the surface profile of the unfired and fired LTCC tapes using a stylus tip surface profilometer. The average surface roughness of the unfired and fired LTCC tapes was found to be $450 \pm 3 \mathrm{~nm}$ and $307 \pm 3 \mathrm{~nm}$ respectively. To understand the effect of surface morphology on NSA several experimental investigations of NSA of proteins as a function of surface roughness exist in literature [12-14]. However, the theory of the kinetics of non-specific adsorption as a function of surface roughness is not known. Therefore, based on the empirical data available, it is hypothesized that the average surface roughness of $1 \mathrm{~nm}$ to $5 \mathrm{~nm}$ promotes non-specific adsorption due to a proportional increase in surface area, as well as creation of nano-cavities and nano-clefts facilitation capture of biomolecules. This particularly applies in this case of Cortisol, which is low molecular weight (362 Dalton), lipophilic molecule. It is also reported that surface roughness of higher magnitude play negligible role in NSA. In the case of LTCC, since the average surface roughness is much greater $\left(\sim 10^{2} \mathrm{~nm}\right)$ than values reported for PDMS, Glass or Silicon, it is expected that the effect of surface roughness on NSA is negligible. 

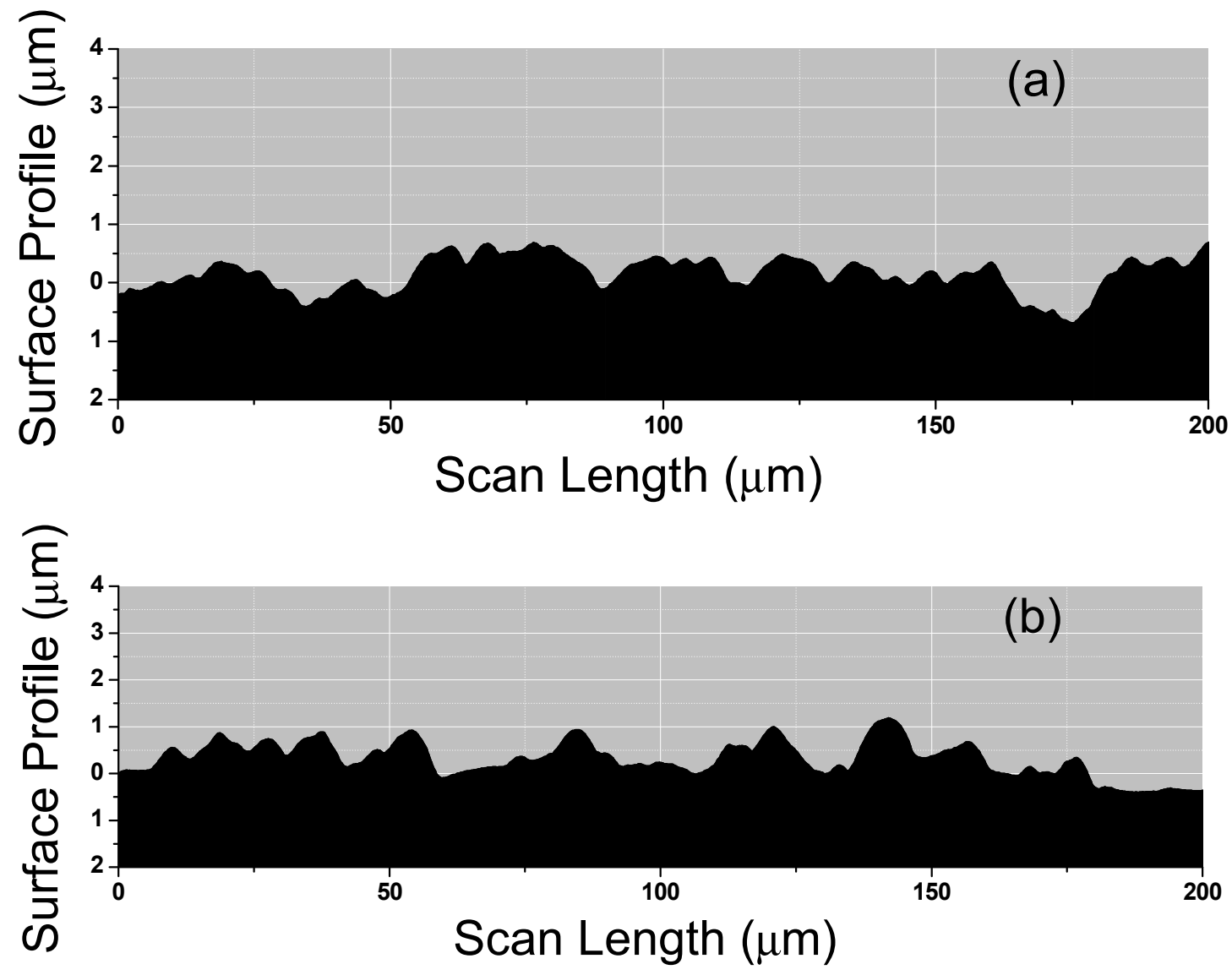

Fig 5.3 Surface roughness profiles obtained for (a) unfired and (b) fired LTCC substrate using a stylus tip profilometer.

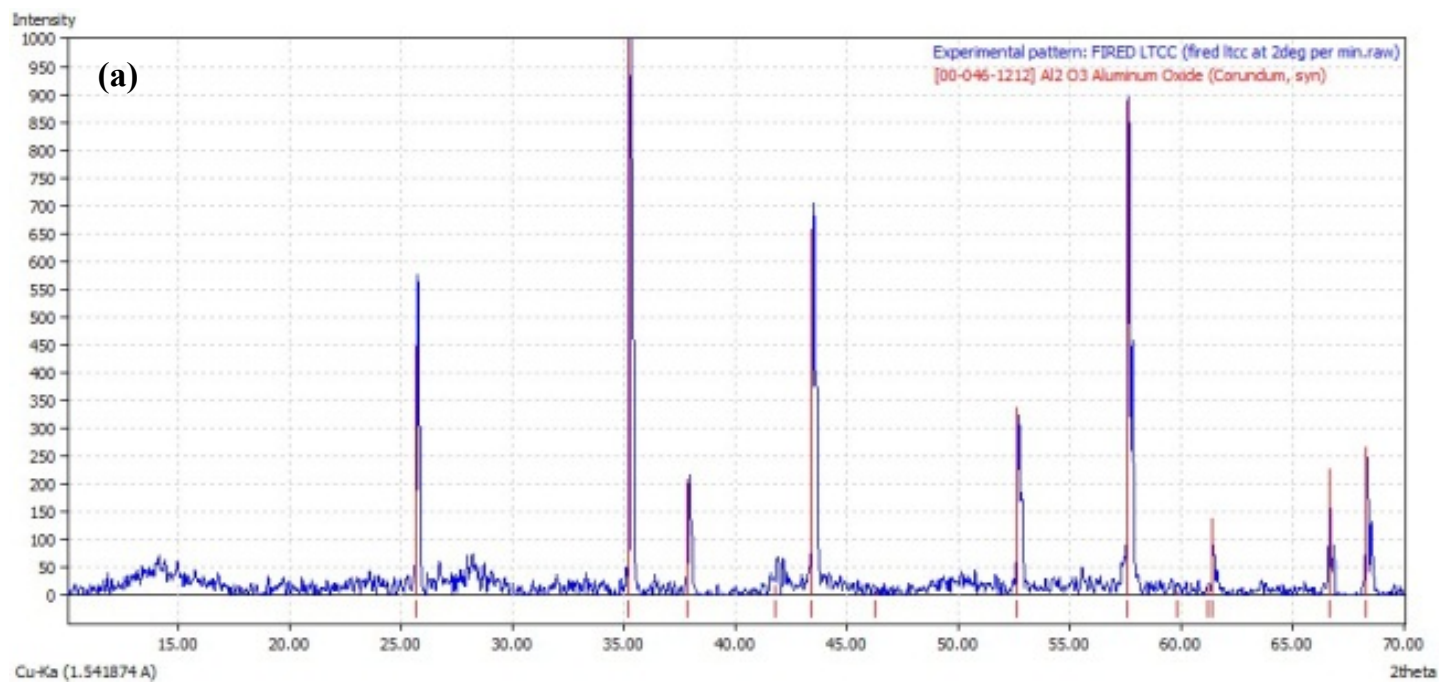

(b) 


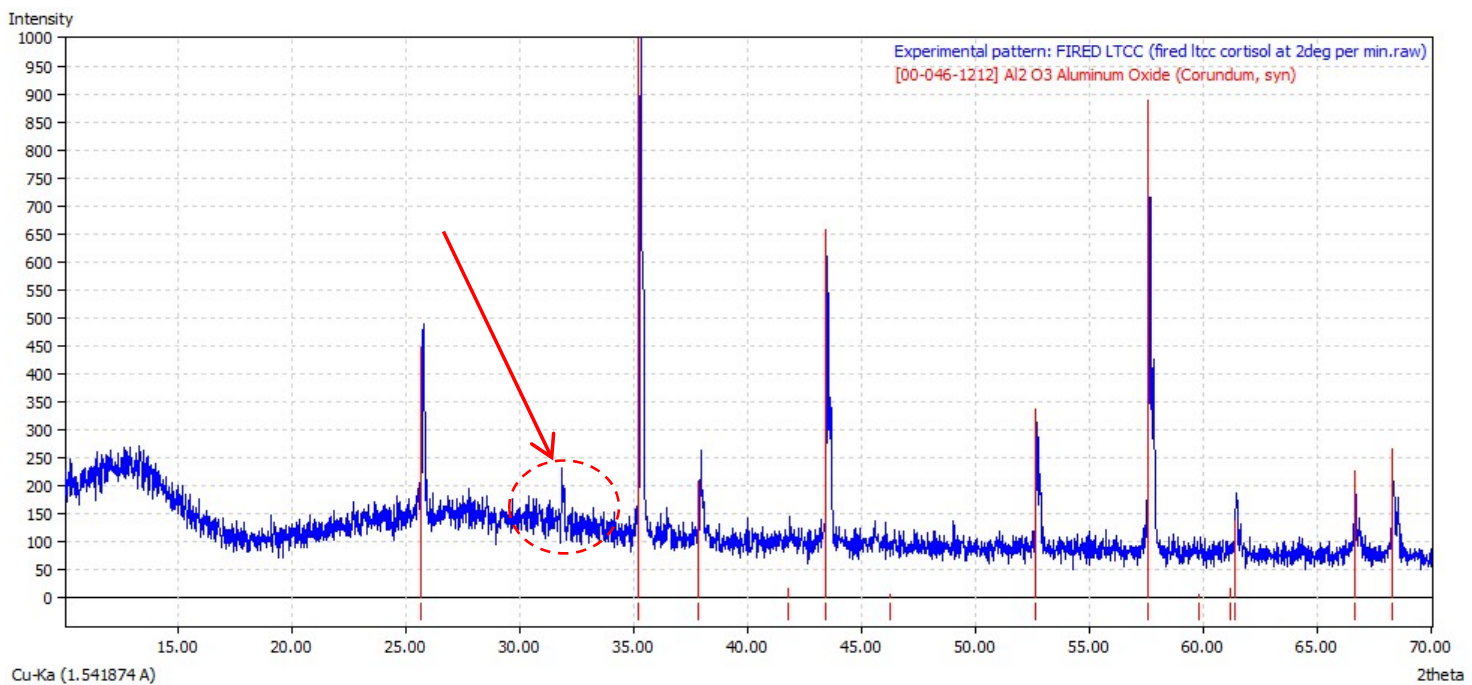

Fig 5.4 X-Ray Diffraction pattern of DuPont 951 LTCC; (a) Fired LTCC; (b) Fired LTCC conjugated with Cortisol.

To characterize the chemical composition of the surface, XRD analysis was performed on fired LTCC samples (Fig 5.4a) and on fired LTCC samples with incubation of Cortisol (Fig 5.4b). Only aluminum oxide peaks were visible and identified in the fired LTCC samples. After incubation of Cortisol, an additional peak was observed (indicated in Fig 5.4b), but the non-crystallinity of Cortisol and high background noise made it difficult to identify the peak as that of a biomolecule such as Cortisol.

The hydrophobicity of a material is directly related to the surface composition and surface energy of a material and is known to be a critical factor in adsorption of biomolecules on a material. Contact angle measurement is a popular technique to characterize adsorption of biomolecules on a surface resulting in increased hydrophobicity[15]. The wetting angle recorded on fired LTCC surface was $26^{\circ} \pm 3^{\circ}$, indicating an intrinsically hydrophilic, high surface energy surface. It is a wellcharacterized phenomenon that protein adsorption decreases as the surface energy of the 
material increases $[16,17]$. No significant change was observed in the contact angle after incubating $5 \mu \mathrm{L}$ of $500 \mathrm{nM}$ cortisol solution for a period of 60 minutes. The wetting angle also has significance in the dynamics of fluid flow in microchannels, and hydrophobicity of LTCC increases the prospects of capillary driven flow in LTCC based microfluidic systems.
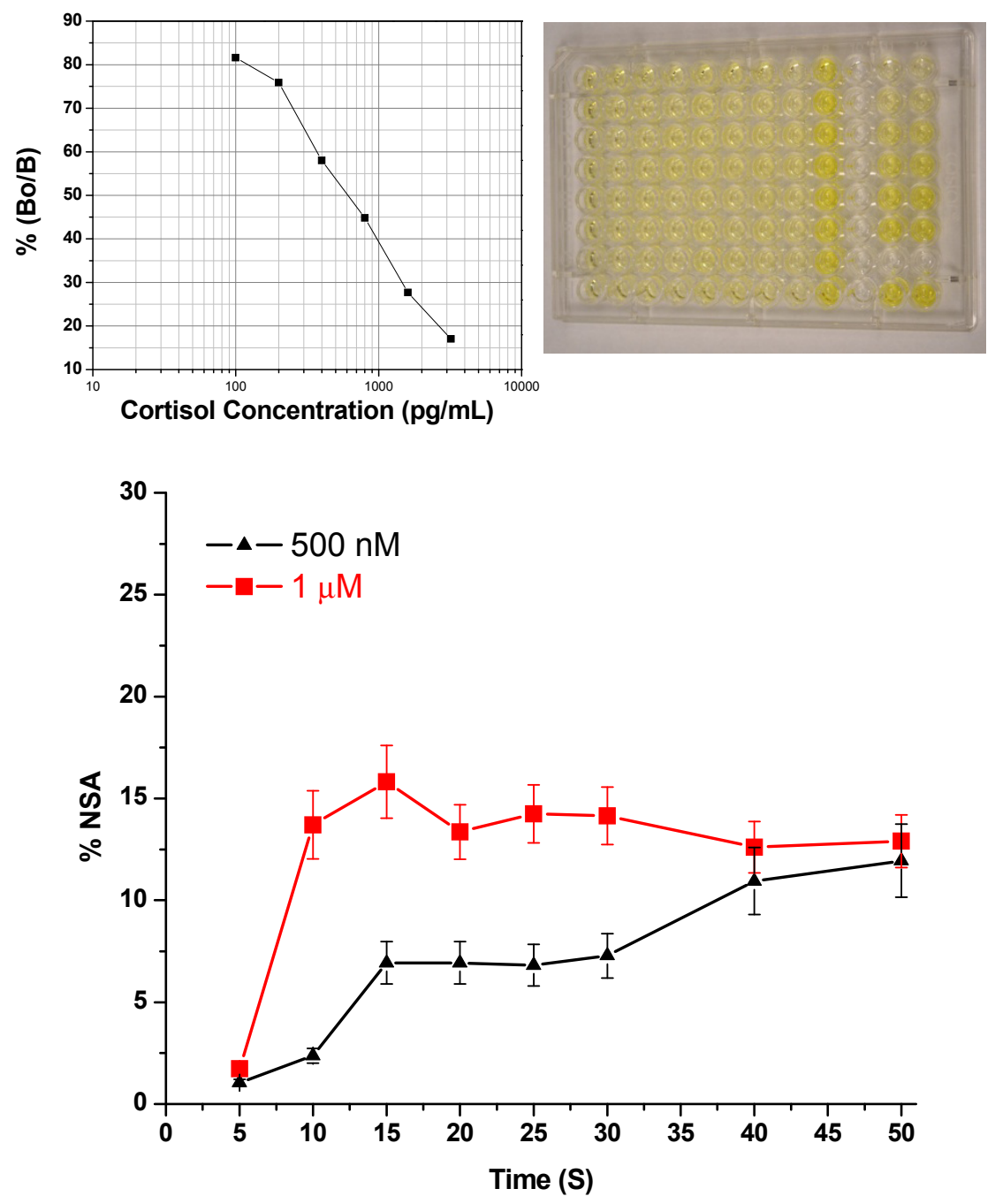

Fig 5.5 (a) ELISA standard curve for cortisol concentration; (b) Picture of the 96 well micro-titer plate after completion of the ELISA assay; (c) \% NSA of $1 \mu \mathrm{M}(-m-)$ and 500nM (- $\mathbf{\Delta}-)$ cortisol solutions incubated on LTCC as a function of incubation time. 
Fig 5.5 presents the percentage non-specific adsorption of cortisol for $1 \mu \mathrm{M}(-\square-)$ and 500nM (- $\mathbf{\Delta - )}$ cortisol solutions incubated on LTCC as a function of incubation time. $10 \mu \mathrm{L}$ of $1 \mu \mathrm{M}$ and $500 \mathrm{nM}$ cortisol solution was incubated in individual wells in the fabricated LTCC micro-well plate (Fig 5.1). At specific time intervals, the cortisol solution was aspirated from the well and the concentration of cortisol was quantified using ELISA. Fig 5.5a presents the standard curve obtained from the ELISA kit. Cortisol concentrations from the NSA study were deduced from this standard curve. Fig 5.5b presents an image of the 96 well micro-titer plate after completion of the ELISA assay. A rapid adsorption of cortisol on LTCC was observed between 5 to 15 minutes, followed by saturation of non-specific adsorption. This effect was more pronounced in the higher concentration $(1 \mu \mathrm{M})$ compared to the lower concentration $(500 \mathrm{nM})$. This may be attributed to concentration dependent kinetics of protein adsorption to reach equilibrium. The adsorption of cortisol was seen to remain constant at around $12 \%$ of the initial concentration for incubation times over 50 minutes. Since the physiological values of cortisol in biofluids such as saliva is many orders of magnitude lower than 500nM and the typical incubation times are generally under 30 minutes, the effect of non-specific adsorption is expected to contribute minimally towards sensor performance.

In this study, properties of LTCC such as surface morphology, surface energy, hydrophobicity and surface roughness, which are responsible for NSA have been characterized. The results show that the NSA is concentration and time dependent. A rapid adsorption was observed initially and a consistent adsorption was observed between 15 to 30 minutes. These finding were factored into during the design of the LTCC microfluidic system and the immunosensing protocol. 


\subsection{Microfluidic Protocol for Electrochemical Immunosensing}

The sequence of operation of the microfluidic manifold to perform the immunoassay is presented below. Fig 5.6 presents a schematic illustration of the microfluidic system design for automation of the immunosensing assay.

1. The sample containing the analyte (cortisol) is introduced into the measurement chamber using an inlet channel at a rate of $10 \mu 1 / \mathrm{min}$ using a programmable syringe pump in the infuse mode. A volume of $10 \mu \mathrm{L}$ is required to fill the measurement chamber and fully dispersed over the functionalized electrode. The sample containing cortisol is allowed to incubate for 30 minutes.

2. A buffer solution of PBS containing $5 \mathrm{mM} \mathrm{Fe}(\mathrm{CN})_{6}^{3-/ 4-}$ is introduced into the chamber at a flow rate of $10 \mu 1 /$ min through the inlet channel. A microfluidic valve is required to switch the inlet channel between the sample introduction pump and the buffer introduction pump. The buffer solution is allowed to flow through the chamber for three minutes $(30 \mu \mathrm{L})$ until the electrode has been washed of all non-specific entities on the surface of the electrode. The buffer and sample exit the chamber through the outlet channel that connects to a waste reservoir.

3. After the washing process, the outlet is closed and $10 \mu \mathrm{L}$ of the buffer solution is allowed to fill the measurement chamber. At this point, all the inlets and outlets are closed and the electrochemical response of the immunoelectrode is measured using a connected Potentiostat. 


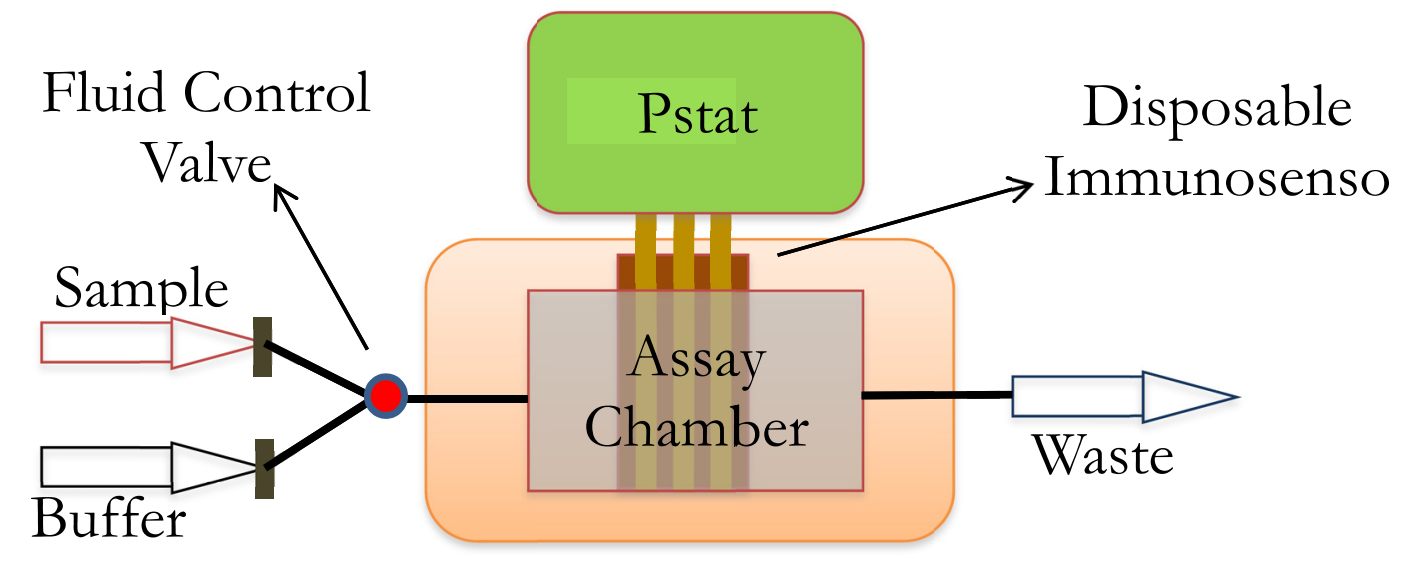

Fig 5.6 Schematic illustration of the microfluidic system for integration with an electrochemical immunosensor

\subsection{Design Optimization Using Computational Fluid Dynamics (CFD)}

An important step in immunosensing assays is the washing step after sample incubation to remove unbound analytes and other non-specific entities from the electrode surface prior to making electrochemical measurements. Opacity of LTCC material hinders the observation of fluid flow; therefore a computational fluid dynamic (CFD) approach has been used to characterize fluid flow profiles to obtain optimum washing efficiency in the assay chamber [18, 19]. CFD approach involves the numerical computation of the interaction of liquids and gases with a material of known boundary conditions (finite element modeling). Numerical modeling in CFD is governed in larger the Navier-Stokes equation, which is derived from the principles of conservation of mass, conservation of momentum and conservation of energy. By incorporating constraints due to physical geometry, flow rate, material and chemical interactions at the solid-liquid-gas boundary line into the numerical model, an accurate prediction of the fluid behavior can be accomplished. All numerical computation presented in this research were performed using COMSOL Multiphysics. Simulation was performed for a two-dimensional, 
incompressible Navier-Stokes flow. A no-slip condition was assumed along the walls of the microchannel and physical values of water (dynamic viscosity: $0.89 \mathrm{cP}$; density: $1,000 \mathrm{~kg} / \mathrm{m}^{3}$ ) were used in the numerical computation. The effect of the position of the inlet and outlet microchannels on the flow profile and the geometry of the assay chamber were investigated. A constant flow rate $(10 \mu \mathrm{l} / \mathrm{min})$ was applied across the cross-section of inlet and outlet microchannels. Fig 5.7a represents the fluid flow trajectories in a square chamber. In the square chamber, the presence of dead volumes was identified along with the formation of vortices, where washing of the electrode would not be effective. Based on these observations, a design modification was incorporated, where the chamber shape was made circular. While dead volumes were eliminated in the circular chamber, it was observed that the fluid flow trajectory was still unevenly distributed across the chamber area (Fig 5.7b). The angles of the inlet and outlet channels were varied and Fig 5.7c presents the optimized design where the fluid flow trajectory was observed to be evenly distributed across the circular chamber. Also, the velocity field and pressure were found to be evenly distributed in the circular chamber. The maximum Reynolds number was computed to be $\sim 0.01$ confirming laminar flow in the microchannels. 

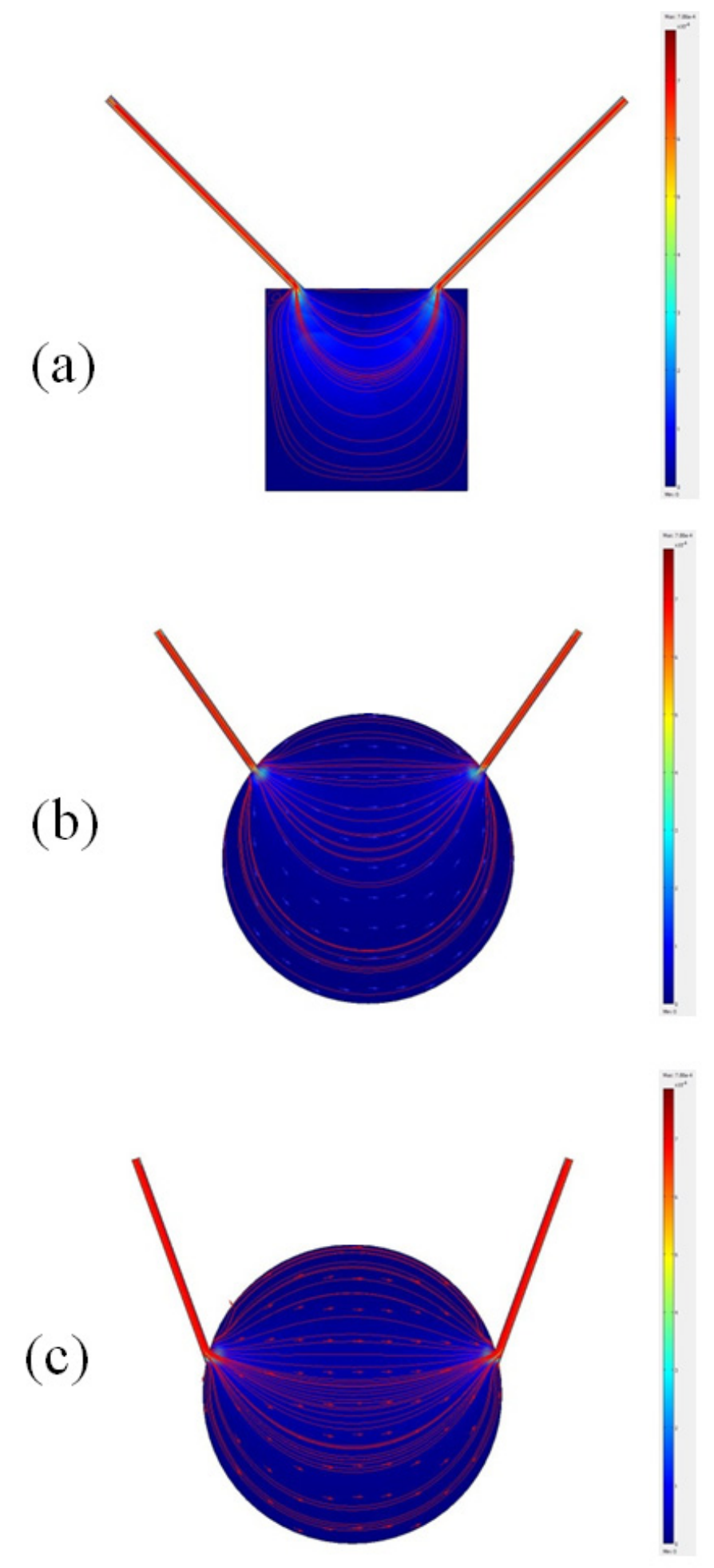

Fig 5.7 Results of CFD simulation in (a) square chamber; (b) circular chamber and (c) circular chamber with inlet and outlet channels are modified position. 


\subsection{LTCC Microfluidic design}

Based on the outcome of the CFD simulation, the optimized design of the microfluidic system is presented in Fig 5.8. The cross sectional schematic (Fig 5.8a) of the microfluidic system depicts the assay chamber, microchannels and the microfabricated sensor chip. The microfluidic chip is designed using three layers of green tape, the design patterns of which are presented in Fig 5.8b. The bottom most layer (i) defines the reaction chamber that interfaces with the sensor chip using a rubber O-ring. The middle layer (ii) defines the inlet and outlet microchannels for sample/buffer introduction and waste removal respectively. The top most layer (iii) provides the cover to the microchannels and ports for incorporating fluidic connectors. A blowout illustration of the three-layer LTCC microfluidic manifold with the sensor chip is presented in Fig 5.8c.

(a)

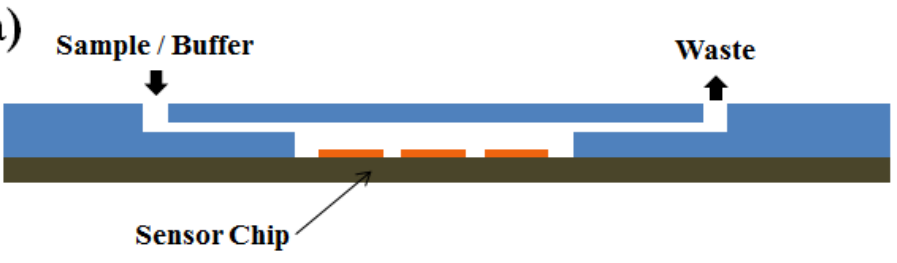

(b)
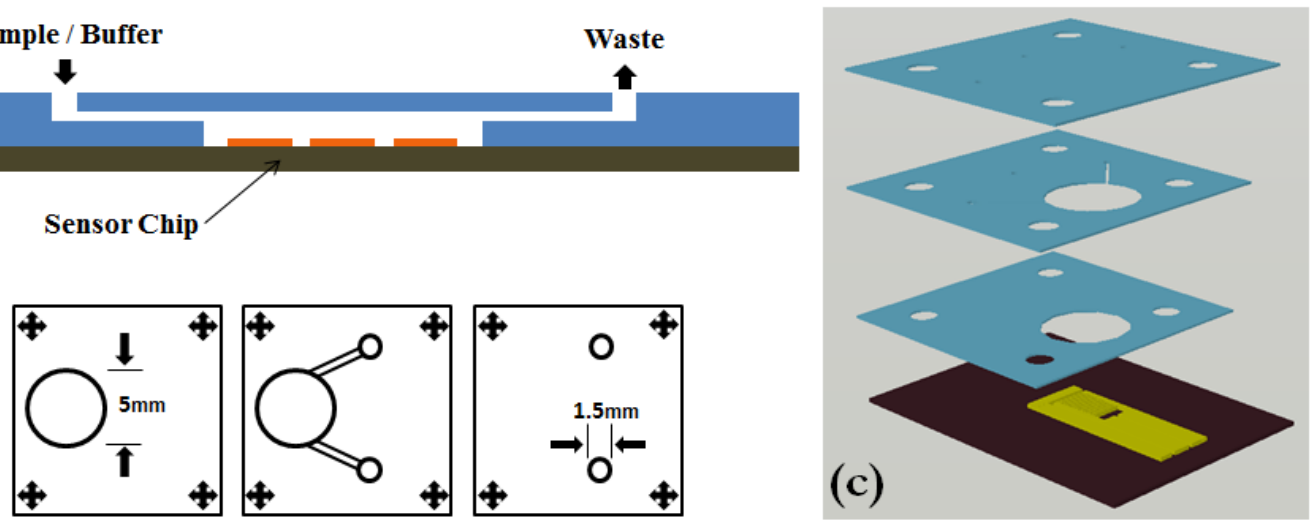

Fig 5.8 Schematic illustration of the LTCC based microfluidic system; (b) The threelayer design of the LTCC microfluidic manifold; (c) A 3D blow out of the microfluidic chip and its integration with the sensor chip. 


\subsubsection{Fabrication of LTCC microfluidic chip}

For fabrication of the LTCC microfluidic manifold, LTCC green tapes (DuPont 951) were purchased from DuPont. The green tapes were patterned using a computer controller $10.6 \mu \mathrm{m} \mathrm{CO}_{2}$ laser (Universal Laser Systems) having a spot size of $50 \mu \mathrm{m}$. The patterned LTCC layers were sequentially aligned using an aligning fixture that was built in-house (Fig 5.9a). The LTCC stack is then laminated using an isostatic hot press (PHITulip) at $150^{\circ} \mathrm{C}$ for 15 minutes at a pressure of 3000 psi. Post-lamination, the LTCC structure was co-fired in an oxygen rich programmable furnace by carefully ramping the temperature up to $850^{\circ} \mathrm{C}$ (Fig $5.9 \mathrm{~b}$ ). Fluidic connectors made from polydimethylsiloxane (PDMS) (Dow Corning) were attached to the inlet and outlet ports of the microfluidic chip and tygon tubing was used to introduce the fluids from a programmable 2-syringe pump network (New Era Pumps). A two-piece acrylic fixture designed and fabricated inhouse was used to integrate the microfluidic manifold with the disposable sensor chip. A rubber O-ring was used to provide a leak-free interface between the sensor chip and the assay chamber.
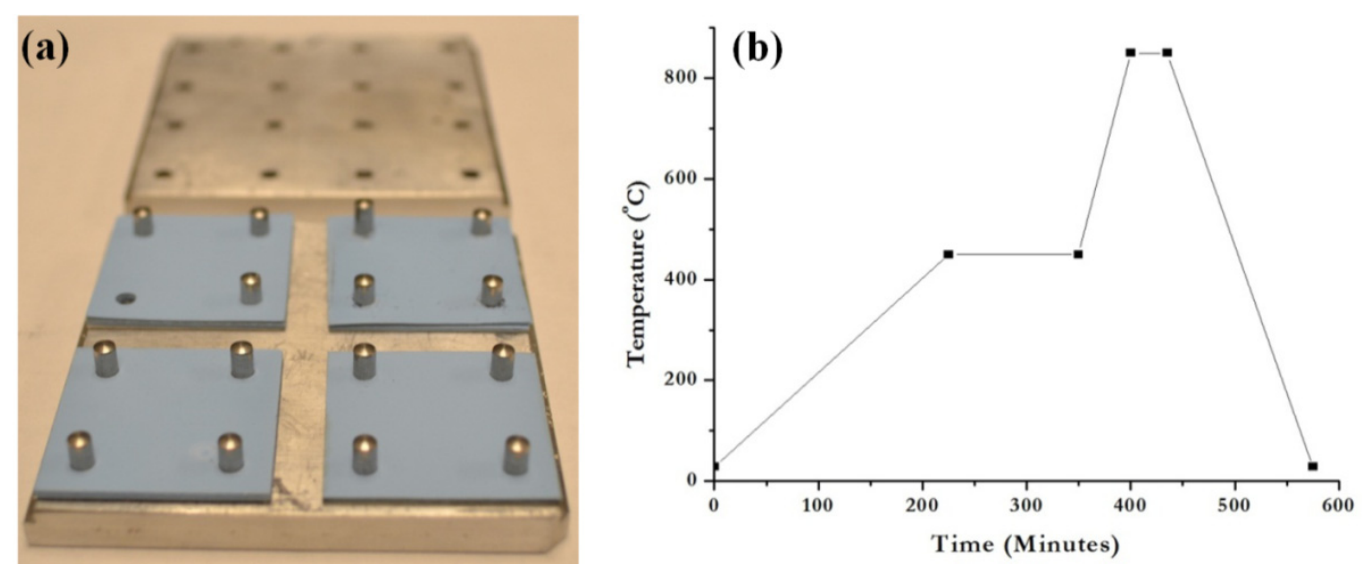

Fig 5.9 Picture of the aligning fixture with LTCC tapes being aligned prior to lamination;

(b) The temperature ramping profile for the sintering process. 
Fig 5.10a presents a picture of the fabricated LTCC microfluidic manifold with connectors and tubing attached. During the lamination stage, no sacrificial material was used to fill the channels to prevent channel sagging[20]. Instead, a sequential lamination process[21], where every layer was sequentially added during the lamination process, was used to obtain sagging and delamination free microchannels. Fig $5.10 \mathrm{~b}$ presents a cross sectional image of the microchannel. The trapezoidal shape of the microchannel cross section is typical of microchannels fabricated using LTCC[21, 22] and results from the excessive burning on only one side of the soft LTCC tapes during laser patterning. The thickness of each individual layer was measured to be $150 \pm 5 \mu \mathrm{m}$ after firing. Due to the trapezoidal cross-section, the width of the channel varied from $100 \pm 5 \mu \mathrm{m}$ at the top to $150 \pm 5 \mu \mathrm{m}$ at the base of the microchannel.
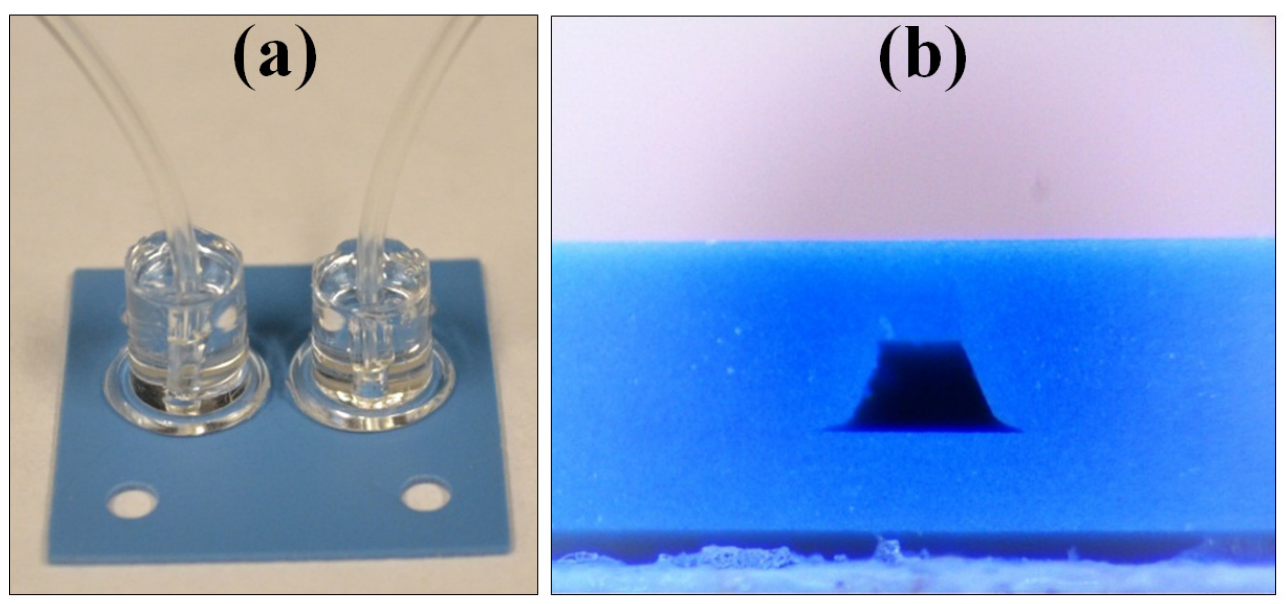

Fig 5.10 (a) The LTCC microfluidic manifold; (b) Cross sectional microscopic image of the LTCC microchannel.

\subsubsection{Fluidic Characterization in LTCC Microfluidic Chip}

For characterizing the fluid flow in the assay chamber, a modified design was utilized, where a glass window was incorporated at the top and bottom of assay chamber 
to visualize the flow of the fluids entering and exiting the assay chamber. Fig 5.11a presents a schematic of the LTCC-glass chip used for fluid flow characterization. Pawel et al have previously demonstrated the process of incorporating glass windows into LTCC structures [23]. Microscope glass cover slips were sandwiched between LTCC layers post-lamination. During the co-firing process, between $650-750^{\circ} \mathrm{C}$, glass softens and bonds to the adjacent LTCC layers, however, the surface tension forces of molten glass assist in maintaining the structure of the glass sheet to accommodate the shrinking of the LTCC structure. Post-sintering clear glass windows were obtained (Fig 5.11b) with no signs of cracking or distortion. To observe the trajectory of fluid flow and to determine the washing efficiency, red color dye solution was used to simulate the sample (contrast) and deionized water (DI) water was used to simulate the washing buffer.

In Fig $5.11 \mathrm{c}$ is presented the snapshots of fluid flow in the microfluidic assay chamber. Fig $5.11 \mathrm{c}(\mathrm{i})$ to Fig $5.11 \mathrm{c}(\mathrm{v})$ presents the introduction of the sample into the assay chamber and Fig $5.11 \mathrm{c}(\mathrm{vi})$ to Fig $5.11 \mathrm{c}(\mathrm{x})$, the washing of the chamber is demonstrated. It can be observed that the trajectory of fluid flow is in accordance with the results obtained from the simulation (Fig 5.7c). Also, the designed flow rate $(10 \mu 1 / \mathrm{min})$ and volume $(30 \mu \mathrm{l})$ of the buffer provided the required washing efficiency. 


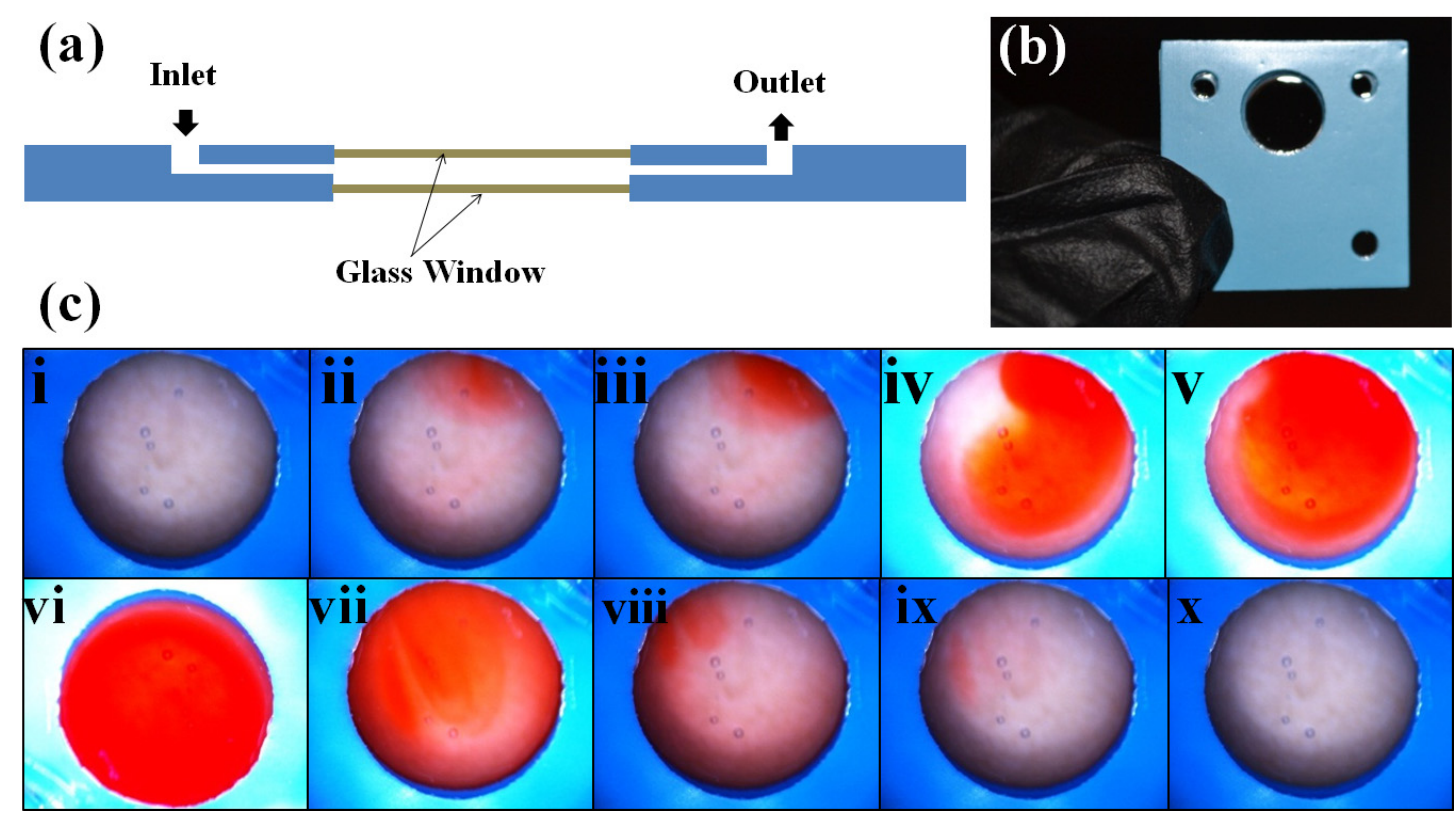

Fig 5.11 (a) Schematic of the LTCC microfluidic chip used for fluid flow characterization; (b) Picture of the glass window integrated into the LTCC chip; (c) Microscopic images of the fluid flow profiles in the assay chamber.

To preferentially select the fluid going into the inlet microchannel, a solenoid three-way valve (The Lee Company) was used. The valve maintains a default path where the fluid flows into valves primary inlet ports and exits through a common outlet port (Fig 5.12a) and upon activation of the valve, fluid flow switches to a secondary inlet port while exiting through the same common outlet (Fig 5.12b). The valve requires a threshold current of $150 \mathrm{~mA}$ and $5 \mathrm{~V}$ to actuate. Fig $5.12 \mathrm{c}$ presents the schematic of the electrical circuit used to actuate the solenoid valve. The circuit uses an opto-isolator to separate the grounds within the same circuit. The opto-isolator provides the necessary current to trigger the transistor to a state of saturation; where in the transistor is used as a switch. The circuit provides to the valve $4.87 \mathrm{~V}$ and $158 \mathrm{~mA}$, which was found to be sufficient to activate the valve. 

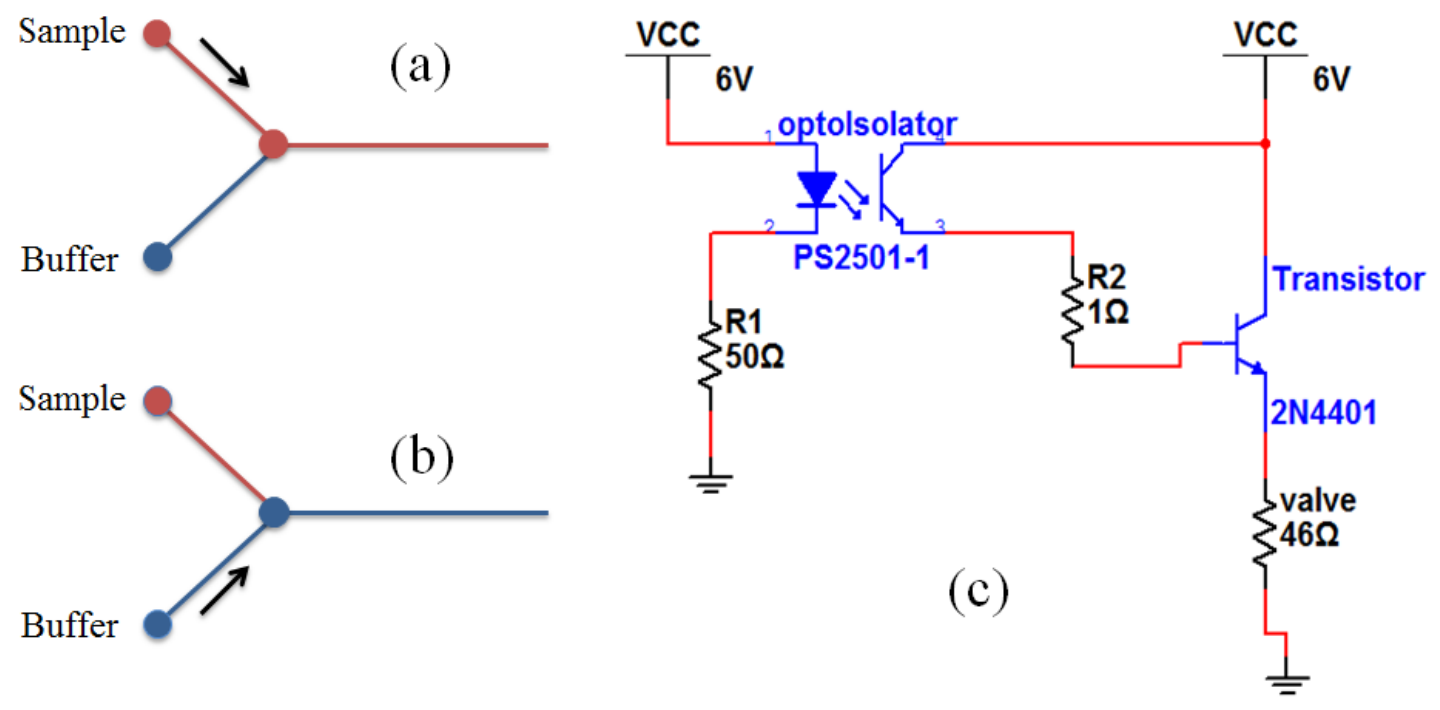

Fig $5.12(a, b)$ Schematic of the operation of the fluidic valve; (c) Schematic of the electronic circuit designed for the activation of the fluidic valve.

The functioning of the fluidic valve was also characterized using two contrast dyes and the functioning of the fluidic valve was verified and is presented in Fig 5.13. In this three-way valve, the common outlet is in the center with each of the two inlets on either side. Under normal conditions (Fig 5.13a), the left input (blue) is open, and the actuation of the valve (Fig 5.13b) switches the flow to the right input (red). A two-piece acrylic fixture designed to house the microfluidic system was fabricated in-house. Fig $5.13 \mathrm{c}$ presents the picture of the testing fixture with the microfluidic chip, the fluid control valve and the disposable sensor chip. All the electrochemical assays as described in Chapter IV were repeated successfully in the fully automated microfluidic system with negligible change in the baselines of the electrochemical response. 

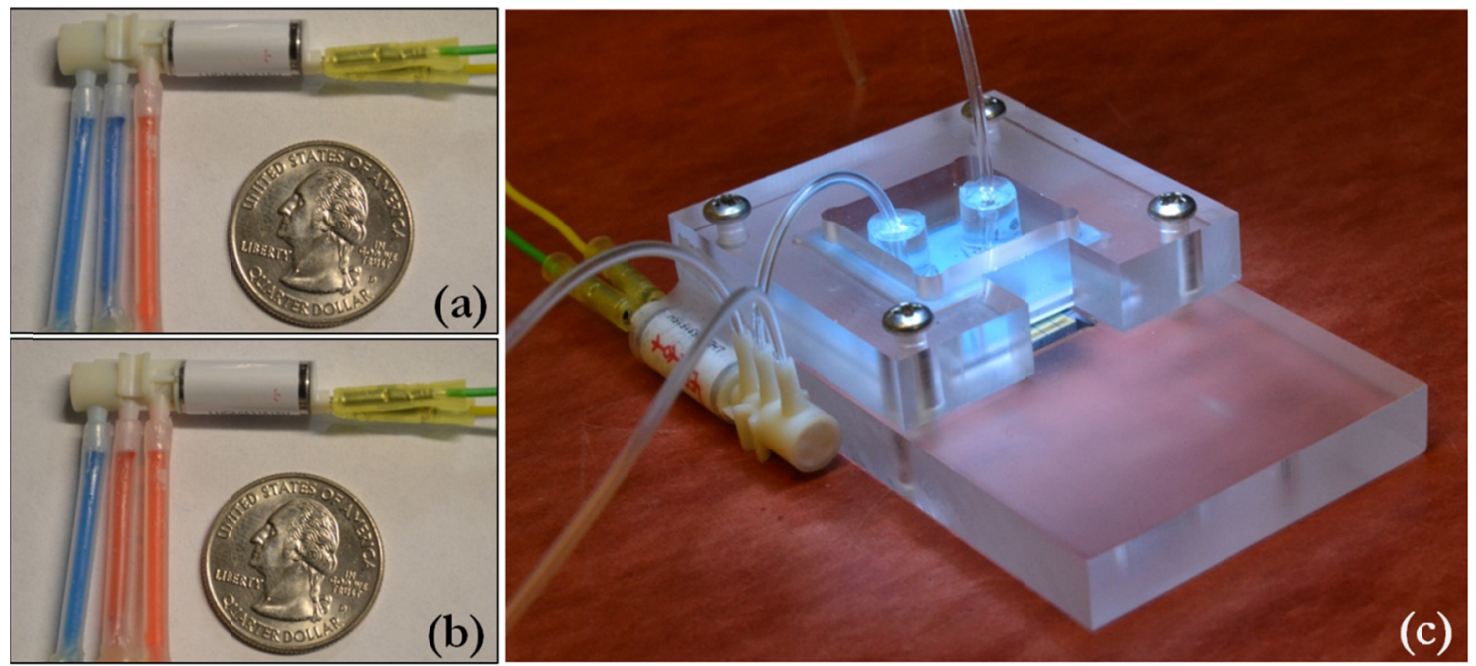

Fig 5.13 (a, b) Characterization of the valving function in the fluidic valve; (c) Picture of the fully integrated microfluidic system with fluid valve, microfluidic manifold and the biosensor chip.

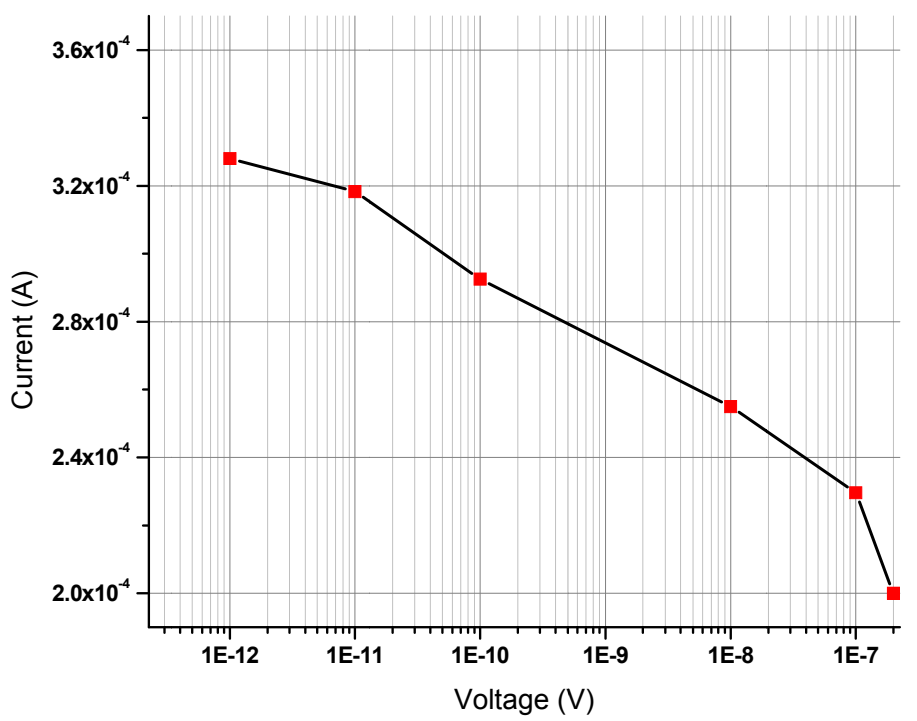

Fig 5.14 Calibration curve obtained from the Electrochemical response studies of EA/Anti-Cab/DTSP/Au immunoelectrode as a function of cortisol concentration (10pM to $200 \mathrm{nM}$ ) in the automated microfluidic cortisol sensor. 


\subsection{Conclusion}

In this chapter, the efforts to develop a low-cost microfluidic system for integrating the developed electrochemical immunosensing platform and the automation of the immunosensing assay are presented. The microfluidic system is developed using LTCC technology, as an alternative to conventional microfluidic systems based on silicon, glass and polymer. For the first time, the non-specific adsorption (NSA) of a biomolecule (Cortisol) on LTCC surface has been characterized as part of the microfluidic system design and development. A CFD approach has been used to optimize the microfluidic geometry with regards to the immunoassay fluidic protocol. The optimized design, fabrication and empirical fluidic characterization of LTCC based microfluidic system is presented. The DTSP-SAM based electrochemical immunosensor is successfully integrated into the microfluidic manifold and the immunosensing assay has been successfully automated for the detection of cortisol. 


\subsection{References}

1. Peterson, S.L., et al., Poly (dimethylsiloxane) thin films as biocompatible coatings for microfluidic devices: cell culture and flow studies with glial cells. Journal of Biomedical Materials Research Part A, 2005. 72(1): p. 10-18.

2. Smetana, W., et al., A multi-sensor biological monitoring module built up in LTCC-technology. Microelectronic Engineering, 2007. 84(5-8): p. 1240-1243.

3. Bartsch de Torres, H., et al., Thick film flow sensor for biological microsystems. Sensors and Actuators A: Physical, 2010. 160(1-2): p. 109-115.

4. William Mercke, T.D., Richard Eitel, Kimberly Anderson. Biocompatibility Evaluation of Human Umbilical Vein Endothelial Cells Directly onto LowTemperature Co-fired Ceramic Materials for Microfluidic Applications. in IMAPS/ACerS 8th International CICMT Conference and Exhibition. 2012. Erfurt, Germany.

5. Zhang, W. and R.E. Eitel, Biostability of Low-Temperature Co-Fired Ceramic Materials for Microfluidic and Biomedical Devices. International Journal of Applied Ceramic Technology, 2012. 9(1): p. 60-66.

6. Zhou, J., A.V. Ellis, and N.H. Voelcker, Recent developments in PDMS surface modification for microfluidic devices. Electrophoresis, 2010. 31(1): p. 2-16.

7. Nicolau Jr, D.V., F. Fulga, and D.V. Nicolau, Impact of protein adsorption on the geometry of microfluidics devices. Biomedical Microdevices, 2003. 5(3): p. 227233.

8. Liu, J., X. Sun, and M.L. Lee, Adsorption-resistant acrylic copolymer for prototyping of microfluidic devices for proteins and peptides. Analytical chemistry, 2007. 79(5): p. 1926-1931.

9. Lasseter, T.L., et al., Covalently modified silicon and diamond surfaces: resistance to nonspecific protein adsorption and optimization for biosensing. Journal of the American Chemical Society, 2004. 126(33): p. 10220-10221.

10. Lee, S.-W. and P.E. Laibinis, Protein-resistant coatings for glass and metal oxide surfaces derived from oligo (ethylene glycol)-terminated alkyltrichlorosilanes. Biomaterials, 1998. 19(18): p. 1669-1675.

11. KUMLANGDUDSANA, P., S.T. DUBAS, and L. DUBAS, Surface Modification of Microfluidic Devices. Journal of Metals, Materials and Minerals, 2007. 17(2): p. 67-74. 
12. Rechendorff, K., et al., Enhancement of protein adsorption induced by surface roughness. Langmuir, 2006. 22(26): p. 10885-10888.

13. Dolatshahi-Pirouz, A., et al., Bovine serum albumin adsorption on nano-rough platinum surfaces studied by QCM-D. Colloids and surfaces. B, Biointerfaces, 2008. 66(1): p. 53.

14. Hovgaard, M.B., et al., Fibronectin adsorption on tantalum: the influence of nanoroughness. The Journal of Physical Chemistry B, 2008. 112(28): p. 82418249.

15. Arima, Y. and H. Iwata, Effect of wettability and surface functional groups on protein adsorption and cell adhesion using well-defined mixed self-assembled monolayers. Biomaterials, 2007. 28(20): p. 3074-3082.

16. Absolom, D., W. Zingg, and A. Neumann, Protein adsorption to polymer particles: role of surface properties. Journal of Biomedical Materials Research, 1987. 21(2): p. 161-171.

17. Jones, M.I., et al., Protein adsorption and platelet attachment and activation, on TiN, TiC, and DLC coatings on titanium for cardiovascular applications. Journal of Biomedical Materials Research, 2000. 52(2): p. 413-421.

18. Malecha, K. and L.J. Golonka. CFD simulations of LTCC based microsystems. in Electronics Technology, 2006. ISSE '06. 29th International Spring Seminar on. 2006.

19. Malecha, K., et al., Low temperature co-fired ceramic (LTCC)-based biosensor for continuous glucose monitoring. Sensors and Actuators B: Chemical, 2011. 155(2): p. 923-929.

20. Birol, H., T. Maeder, and P. Ryser, Application of graphite-based sacrificial layers for fabrication of LTCC (low temperature co-fired ceramic) membranes and micro-channels. Journal of Micromechanics and Microengineering, 2007. 17(1): p. 50.

21. Farhan Shafique, M., et al., Fabrication of embedded microfluidic channels in low temperature co-fired ceramic technology using laser machining and progressive lamination. Journal of the European Ceramic Society, 2011. 31(13): p. 21992204.

22. Barlow, F., et al., Fabrication of Precise Fluidic Structures in LTCC. International Journal of Applied Ceramic Technology, 2009. 6(1): p. 18-23.

23. Bembnowicz, P., D. Nowakowska, and L. Golonka. Integrated LTCC-glass 
microreactor and \&\#x00B5;TAS with thermal stabilization for biological application. in Microelectronics and Packaging Conference, 2009. EMPC 2009. European. 2009. 


\section{CHAPTER VI}

\section{Summary and Future Direction}

\subsection{Summary}

In summary, this dissertation has detailed the development of a label-free, electrochemical immunosensing platform integrated into a low-cost microfluidic system for the sensitive, selective and accurate detection of cortisol, a steroid hormone co-related with many physiological disorders. Abnormal levels of cortisol is indicative of conditions such as Cushing's syndrome, Addison's disease, adrenal insufficiencies and more recently post-traumatic stress disorder (PTSD). Electrochemical detection of immunocomplex formation has been utilized for the sensitive detection of Cortisol using AntiCortisol antibodies immobilized on sensing electrodes. Electrochemical detection techniques such as cyclic voltammetry (CV) and electrochemical impedance spectroscopy (EIS) have been utilized for the characterization and sensing of the labelfree detection of Cortisol. The utilization of nanomaterials as the immobilizing matrix for Anti-cortisol antibodies that leads to improved sensor response has been explored.

The first research activity undertaken was to establish an immunosensing platform for the electrochemical detection of cortisol. A nanostructured immunosensing platform based on a hybrid nanocomposite film of core-shell $\mathrm{Ag} @ \mathrm{AgO}$ nanoparticles and Polyanaline (PANI) has been synthesized using electrophoretic deposition (EPD) onto Au substrate. The core-shell Ag@AgO nanoparticle $(\sim 5 \mathrm{~nm})$ has successfully been embedded in PANI backbone to make a highly electro-active Ag@AgO-PANI nanocomposite. For the first time, the electrophoretically deposited nanomaterial was utilized for the immobilization of monoclonal Anti- $\mathrm{C}_{\mathrm{ab}}$ to fabricate a label-free and 
mediator free electrochemical immunosensor for cortisol detection. Results of electrochemical characterization suggest that $\mathrm{Ag} @ \mathrm{AgO}$ nanoparticle act as doping agents for PANI and result in the increase charge density and stabilize electrochemical behavior, resulting in enhanced charge transfer from redox moieties to electrode. This increased electro-active platform with nano-porous morphology and functionality provides a friendly environment to immobilize Anti- $\mathrm{C}_{\mathrm{ab}}$. The immunosensor was tested as a function of cortisol concentrations in phosphate buffer saline (PBS) using cyclic voltammetry and found to exhibit a detection limit of $0.64 \mathrm{pg} \mathrm{mL}^{-1}$ (estimated using $3 \sigma$ formula), a linear range from $1 \mathrm{pM}$ to $1 \mu \mathrm{M}$, with a sensitivity of $66 \mu \mathrm{A} / \mathrm{M}$.

The ultimate goal of this research was to develop a point-of-care immunosensing platform for the detection of cortisol. Hence next logical step was to integrate the immunosensing platform onto a micromachined co-planar electrode set-up. The developed Ag@AgO-PANI based immunosensor was unable to be successfully integrated onto microfabricated electrodes due to a high degree of non-specific electrophoretic deposition of the hybrid nanocomposite onto the base substrate (Glass) leading to electrical shorting of the fabricated electrodes. To overcome challenges related to system integration, an alternate strategy, involving the use of self-assembled monolayer of DTSP as the nanostructured matrix for the immobilization of anti-Cortisol antibodies was explored. The DTSP-SAM was modified onto microfabricated interdigitated microelectrodes ( $\mu \mathrm{IDE}$ ). Electrochemical characterization of the DTSPSAM modification and fabrication of the immunoelectrode is presented. The immunosensor was tested as a function of cortisol concentrations in phosphate buffer saline (PBS) using cyclic voltammetry and found to exhibit a detection limit of $10 \mathrm{pM}$, a 
linear range from $10 \mathrm{pM}$ to $200 \mathrm{nM}$, with a sensitivity of $20.7 \mu \mathrm{A} / \mathrm{M}$. The next research activity planned was to automate the electrochemical immunoassay as a step towards realizing a fully automated point-of-care cortisol sensor. The microfluidic system is developed using LTCC technology, as an alternative to conventional microfluidic systems based on silicon, glass and polymer. For the first time, the non-specific adsorption (NSA) of a biomolecule (Cortisol) on LTCC surface has been characterized as part of the microfluidic system design and development. A CFD approach has been used to optimize the microfluidic geometry with regards to the immunoassay fluidic protocol. The optimized design, fabrication and empirical fluidic characterization of LTCC based microfluidic system is presented. The DTSP-SAM based electrochemical immunosensor is successfully integrated into the microfluidic manifold and the immunosensing assay has been successfully automated for the detection of cortisol. The developed sensor holds potential for application in sensing of cortisol in real-time and at point-of-care. The future prospects is presented in the next section.

\subsection{Future Direction}

The future direction for the developed microfluidic cortisol sensing system is envisioned in two directions, a brief description of which is provided here.

1. Wearable sensors for monitoring of stress related disorders

Lifestyle related health issues have gained attention and focus over the last decade due to increasing effects of day to day activities on general health. One such serious issue is psychological stress experienced by individuals on a daily basis. The human body reacts to stress in different ways, of which, the release of 
hormones and neurotransmitters is a typical response. Prolonged exposure to stress leads to the activation of signaling pathways from the brain that leads to release of cortisol from the adrenal cortex. Cortisol secretion levels have been correlated to stress levels in patients suffering from stress disorders such as posttraumatic stress disorder (PTSD), insomnia and burnouts. PTSD affects about 24 million people in the Unites States alone with $30 \%$ of combat veterans having been reported with PTSD. The sensing platform reported in this dissertation for detection of cortisol has the potential to be integrated into a wearable system for online and continuous monitoring of cortisol levels as a function of one's environment. This Cortisol sensor, coupled with a continuous biofluid harvesting system, could allow a continuous readout of Cortisol levels in the ambulatory setting, providing real-time information on cortisol secretion values when a patient is subjected to psychological stress as a resulting of triggers from the surroundings and situations. The information arising from the data could be used to understand behavioral patterns and also could be used to create personalized and targeted medications for stress related disorders. The continuous monitoring system may also provide information on the 24-hour diurnal cortisol at the night time minimum value and can identify the precise point in the sleep cycle at which Cortisol begins its morning cycle. It could provide easy access to the postawakening daily peak of Cortisol production as well.

\section{Disposable Microfluidic Cortisol Sensor for point-of-care diagnostics}

A novel microfluidic system design has been envisioned that incorporates the 
developed electrochemical cortisol immunosensor into a single use disposable strip. The system design is based on use of passive microfluidic principles such as capillary driven flow. The analysis unit, into which the sensor strip can be inserted, can contains a low-power potentiostat along with all the necessary automation that is required for sample and reagent processing. A schematic of the disposable cartridge and the corresponding analysis unit is presented in Fig 6.1.

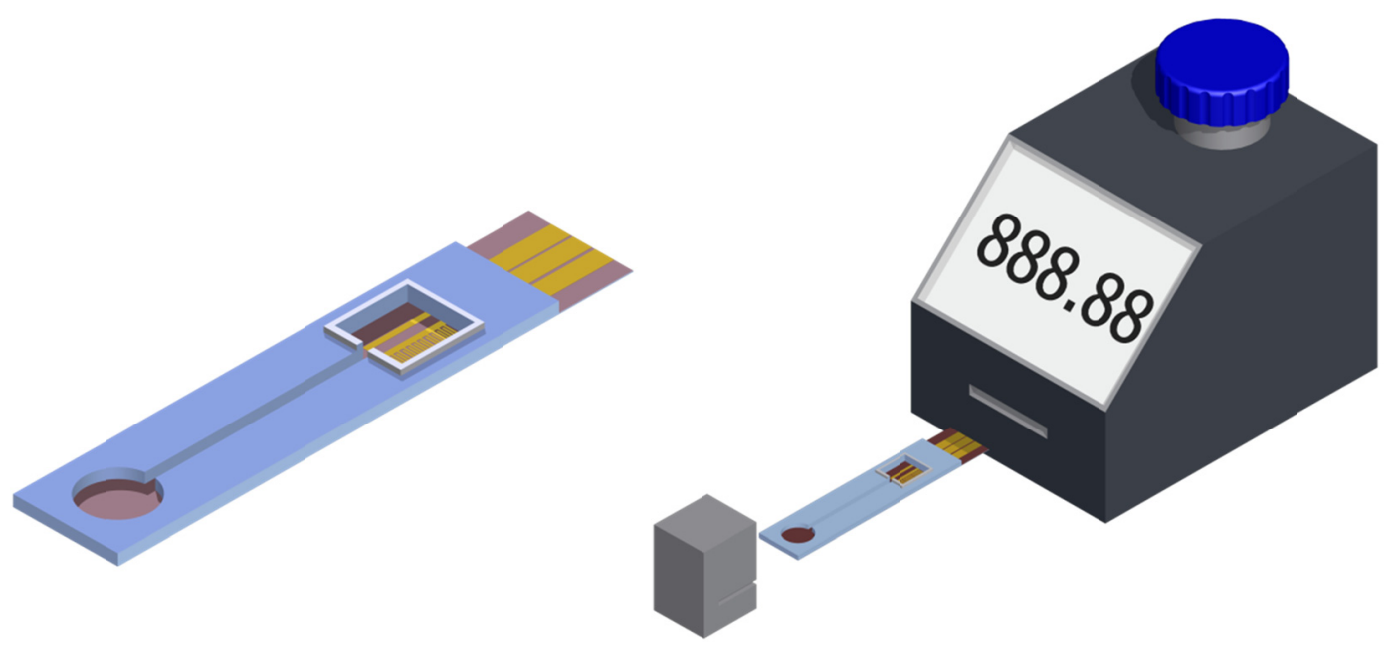

Fig 6.1 Envisioned hand-held/bench-top cortisol detection system. 
VITA

\section{ABHAY VASUDEV}

Born, Hyderabad, India

2002-2006

B.E., Instrumentation Technology

J. S. S. Academy of Technical Education

Visveswariah Technological University

Bangalore, India

2006-2009

M.S., Mechanical Engineering

University of Akron

Akron, Ohio

$2010-2013$

Doctoral Candidate

Electrical and Computer Engineering

Florida International University

Miami, Florida

\section{BOOK}

1. Shekhar Bhansali, Abhay Vasudev, "MEMS for Biomedical Applications", Woodhead Publishing, July 2012. ISBN 0857091298

\section{BOOK CHAPTER}

1. Abhay Vasudev, Shekhar Bhansali, "MEMS for in-vivo applications", in "Implantable sensor systems for medical applications", Edited by Andreas Inmann and Diana Hodgins, Woodhead Publishing, Jan 2013. ISBN 1845699874

\section{JOURNAL PUBLICATIONS}

1. Abhay Vasudev, Ajeet Kaushik, Shekhar Bhansali, "Non-Specific Adsorption (NSA) of Cortisol on LTCC Towards Developing LTCC Microfluidic Cortisol Immunosensor", Submitted to Applied Surface Science, April 2013.

2. Ajeet Kaushik, Abhay Vasudev, Sunil Arya, Shekhar Bhansali, "High sensitive, label free electrochemical immunosensor for cortisol detection using electrophoretically deposited polyanaline-Ag/AgO nanoparticles", Submitted to Biosensors and Bioelectronics, March 2013.

3. Ajeet Kaushik, Sunil Arya, Abhay Vasudev, Shekhar Bhansali, "Recent Advances in Detection of Ochratoxin-A", Accepted to Open Journal of Applied Biosensor, 2013, Vol. 2, Issue 1, Pages 139-146. 
4. Ajeet Kaushik, Sunil Arya, Abhay Vasudev, Shekhar Bhansali, "Nanocomposites based on Chitosan-Metal/Metal Oxide Hybrids for Biosensors Application", Journal of Nanoscience Letters, Jan, 2013, (In Press)

5. Abhay Vasudev, Yuichi Tomizawa, Ajeet Kaushik, Shekhar Bhansali, "An LTCC based microfluidic electrochemical biosensing system for label-free detection of cortisol", Sensors and Actuators: B Chemical, 2013, Vol. 182, Pages 139-146.

6. Abhay Vasudev, Ajeet Kaushik, Kinzy Jones, Shekhar Bhansali, "Prospects of Low Temperature Co-fired Ceramic (LTCC) based microfluidic systems for point-of-care biosensing and environmental sensing", Microfluidics and Nanofluidics, 2012, Vol. 14, Issue 3-4, Pages 683-702.

7. Abhay Vasudev, Ajeet Kaushik, Shekhar Bhansali, "Ultrasensitive Electrochemical Immunosensor for Epidermal Growth Factor Receptor (EGFR) Detection", 2013, Vol. 39, Issue 1, Pages 300-305.

\section{CONFERENCE PRESENTATIONS}

1. Abhay Vasudev, Ajeet Kaushik, Shekhar Bhansali, "Characterization of the nonspecific adsorption of cortisol on LTCC", IMPAS Ceramic Interconnects and Ceramic Microsystems Technology (CICMT), Orlando, FL, April 23-25, 2013 (Oral)

2. Abhay Vasudev, Ajeet Kaushik, Shekhar Bhansali, "Ultrasensitive Electrochemical Immunosensor for Epiderman Growth Factor Receptor (EGFR) Detection", World Congress on Biosensors (Biosensors), Cancun, Mexico, May 15-18, 2012 (Oral)

3. Ajeet Kaushik, Abhay Vasudev, Sunil Arya, Shekhar Bhansali, "Highly Sensitive, Label Free Immunosensor to Detect Cortisol Using Electrophoretically Deposited Ag@AgO-Polyaniline Nanocomposite", NanoFlorida, Tampa, FL, September 28-29, 2012 (Poster)

4. Shekhar Bhansali, Abhay Vasudev, Ajeet Kaushik, Sunil Arya, Manju Venugopal, "SAM Modified IDE for Impedimetric Immunosensor for Detection of Cortisol", Indo-Japan Workshop on Biomolecular Electronics (IJWBME), December 7-10, 2011 (Oral)

5. Abhay Vasudev, Sunil Arya, Shekhar Bhansali, "A Real-time, Automated Microfluidic System for Cortisol Detection”, NanoFlorida, Miami, FL, September $30^{\text {th }}$ - October 1 $1^{\text {st }}, 2011$ (Poster) 